\title{
The First Spectrum of Manganese, Mn I
}

\author{
Miguel A. Catalán, ${ }^{1}$ William F. Meggers, ${ }^{2}$ and Olga Garcia-Riquelme ${ }^{3}$
}

\author{
June 29, 1963
}

\begin{abstract}
In 1894, two short series of threefold spectral terms were discovered in the arc spectrum of manganese, and in 1922 other regularities involving fivefold and sixfold terms were discovered by Catalán who coined the word "multiplet" for the group of related lines resulting from combinations of such complex terms. Multiplet analyses of complex spectra promptly led to the present formal quantum interpretation of all such phenomena, but comparable progress in the analysis of the MnI spectrum was handicapped by the paucity of experimental data.

New observations of about 2500 wavelengths and intensities plus 440 Zeeman patterns made available in 1948-49 have now been completely exploited to derive additional atomic energy levels and thereby explain more of the observed Mn I lines. The result is that a total of 42 even terms with 125 levels and $60 \mathrm{~g}$-values have now been designated and allocated to electron configurations, and 94 odd terms with 266 levels, $164 g$-values, plus 13 miscellaneous levels. These terms are distributed among four multiplicities (doublets, quartets, sextets, octets), and transitions between even and odd terms account for more than 2030 lines ranging in wavelength from $1785 \AA$ to $17608 \AA$.
\end{abstract}

\section{Introduction}

The spectra of manganese have interested and engaged scientists for nearly 120 years. In 1910 Kayser [1] ${ }^{4}$ listed 67 publications on this subject since 1845, and in 1934 Kayser and Konen [2] compiled a bibliography of 154 additional items through 1931. In the present paper only a few of the above will be referred to for historical background, but most of the papers published since 1931 that describe or interpret the Mn I spectrum will be mentioned to extend the bibliography.

In 1894 Kayser and Runge [3] reported finding the first regularities among lines in the arc spectrum of manganese; they found five triplets with wave number differences of 173 and $129 \mathrm{~K}$ ( $\mathrm{K}=$ kayser $=1$ $\mathrm{cm}^{-1}$ ) and arranged them in two spectral series, one "sharp" and one "diffuse," like those in the simpler spectra of alkaline-earth elements. (This precious information was presented in a footnote on page 104 of a paper primarily dedicated to the spectra of tin, lead, arsenic, antimony, and bismuth.)

Nearly three decades after Kayser and Runge [3] discovered the first rudimentary series in the are spectrum of manganese, Catalán [4] extended those series and calculated the first ionization potential of manganese as 7.41 electron volts. Catalán said (p. 146) "Whilst the manganese series of ordinary type were under investigation, it was noted that there was a strong tendency for lines of similar character to appear in groups (of 9, 12, or 15 lines) and that such groups included some of the most intense lines in the spectrum". For such a group of related spectral lines, "multiplet" was suggested

\footnotetext{
Institute of Optics, Madrid. Died 11 November 1957. This report has been prepared by the surviving authors to honor their deceased colleague.

2 National Bureau of Standards, Washington.

3 Institute of Optics, Madrid.

4 Figures in brackets indicate the literature references at the end of this paper.
}

(p. 147). "The accuracy of the separations, some of them being identical with those of the ordinary series, together with the fact that the lines of each group are of the same character, strongly suggests that the multiplets have a real physical significance. Further evidence for their reality is afforded by the occurrence of similar multiplets in the spectra of other elements" (p. 163). In fact, Catalán's first paper on regularities in the spectrum of manganese reporting triplets and multiplets that reveal terms with five or six levels [4] also reported three multiplets in the spectrum of ionized manganese and three multiplets of the neutral atom of chromium. Even before that paper was printed, a manuscript copy of it inspired Sommerfeld [5] to write on the explanation of complex spectra (manganese, chromium, etc.) according to the method of inner quantum numbers. Almost simultaneously, Back [6] accurately measured the Zeeman effect of 49 lines in the arc- and 12 lines in the spark spectrum of manganese with the object of investigating magnetic term splitting of the complex terms found by Catalán, and Landé [7] provided an explanation of all Zeeman patterns with the aid of magnetic quantum numbers and experimental magnetic splitting factors for different types of spectral terms and multiplicities.

It was then clear that the regularities among 169 lines of Mn I presented by Catalán [4] arose from spectral terms belonging to sextet and octet systems. As a check of Landé's scheme, and an example of the usefulness of Zeeman effect in analyzing complex spectra, Back [6] found from Zeeman data the first multiplet of the quartet system. Since multiplets usually occur in triplicate $(\Delta l=0, \pm 1)$, Catalán [8] promptly found two additional quartet multiplets associated with the one described by Back.

In 1926 McLennan and McLay [9] extended the work of Catalán and Back on Mn I by making absorption experiments, finding 14 additional multi- 
plets, providing energy diagrams for quartets, sextets, and octets, and tables of 90 energy levels and 257 classified lines ranging in wavelength from $1874.7 \AA$ to $17607.5 \AA$. Although terms of doublet multiplicity were sought, none was found at that time. This list of levels and terms, with some additions from Russell [10] and from Catalán [11], and with modernized notation, appeared in a volume of Atomic Energy States published in 1932 by Bacher and Goudsmit [12]; it presented 99 energy levels distributed in 17 quartet, 16 sextet, and 15 octet, terms then recognized for the Mn I spectrum. The following year, T. Dunham, Jr., [13] found ten new quartet and two sextet terms (but never published them), and Meggers [14] detected one quartet and two sextet terms in new observations of near infrared spectra. Aside from the above, fifteen years passed without progress in the analysis of Mn I. A strong incentive to extend this analysis came in 1946 when C. E. Moore began her critical and comprehensive compilation of Atomic Energy Levels as Derived from the Analyses of Optical Spectra.

In 1949 a major advance in the analysis of Mn I was made by Miss Olga Garcia-Riquelme [15] who was assigned the task as a thesis problem by Professor Catalán at the University of Madrid. By assembling all known energy levels of Mn I and by compiling wavelengths and intensities of manganese lines from many spectroscopic papers (by 15 different authors), Garcia-Riquelme succeeded in finding 58 new energy levels and 15 new terms for Mn I, thus producing a total of 30 even terms (85 levels), 30 odd terms (124 levels), plus 21 miscellaneous odd levels beyond the ionization limit. The permitted transitions between these levels accounted for 711 observed lines ranging in wavelength from 1876.48 A to $17607.5 \AA$. All the established spectral terms of Mn I were assigned to quartet, sextet, and octet systems; doublet terms were still unrecognized.

In order to complete the bibliography on description and interpretation of Mn I spectra during the period 1931 to 1949 , we mention a paper by Slevogt [16] and one by Paul [17]. The former quotes earlier data on 233 lines (between 6024.67 and $8933.03 \mathrm{~A}$ ) and remeasured the wavelengths of 113 of these; the latter reported observations on $57 \mathrm{Mn}$ I lines with wavelengths between 1923.05 $\AA$ and $1085.01 \AA$ absorbed by manganese vapor. Paul explained 10 of these as transitions from the ground state to highly excited levels, but only three of these have been confirmed in this analysis of $\mathrm{Mn} \mathrm{I}$.

In 1948 Catalán decided that an improved description of the Mn I spectrum (including reliable wavelengths, realistic relative intensities for several thousand lines, and Zeeman data for hundreds of lines) was prerequisite to a more complete and satisfactory term analysis of this spectrum.

\section{Experiments}

To improve the data of Mn I Catalán spent most of 1948 in the United States, where he recorded the vacuum ultraviolet arc spectrum of manganese at
Princeton University, and photographed the arc spectrum throughout the visible and adjacent ultraviolet at the National Bureau of Standards.

The vacuum ultraviolet are and spark spectra of manganese were photographed with a two-m radius grating at Princeton University through the courtesy of Allen G. Shenstone who previously described [18] the apparatus and procedure. The manganese spectra were recorded in this vacuum spectrograph from $1460 \AA$ to $2180 \AA$ with a scale of $4.8 \AA$ per mm, and all these spectrograms were measured and converted to wavelengths by Catalán.

In 1933, Meggers photographed and measured an ultraviolet range $(2100 \AA$ to $2800 \AA)$ of manganese spectra with the largest quartz Littrow spectrograph constructed by Adam Hilger, Ltd., of London. Provided with a quartz lens of three-m focal length and Cornu prisms of $60-\mathrm{cm}$ total base, this spectrograph produced reciprocal dispersions of 0.4 to $1.0 \AA$ / $\mathrm{mm}$ at the above-mentioned wavelengths. These results were presented to Catalán in 1948 in addition to several dozen new lines between $10500 \AA$ and 12000 A recorded by Meggers on Eastman 1-Z photographic plates to extend his earlier observations [14] made with Eastman 1-Q plates.

In 1948 Meggers and Catalán reobserved the arc and spark spectra of manganese between $2200 \AA$ and $7800 \AA$ with the aid of a concave grating supplied by R. W. Wood [19]. In a Wadsworth-type mounting, this grating of 30,000 lines per inch on aluminized pyrex glass with 22 feet radius of curvature provided ghost-free spectra with reciprocal dispersions of 1.7 to $2.4 \AA$ per $\mathrm{mm}$ in the first order. The second order spectra with double dispersion were photographed between $2500 \AA$ and $4500 \AA$. All these spectrograms were measured relative to international standards of wavelength provided by the iron arc, and improved wavelengths and estimated intensities of more than 2000 lines characteristic of Mn I were thus obtained in 1949. Excepting several hundred wavelengths since reported in papers on the Zeeman effect, none of these new data has been published heretofore. To complete this description of the Mn I spectra, we (like others) have quoted 16 infrared wavelengths $(12900 \AA$ to $17608 \AA$ ) emitted by ares of 60 to 80 amperes and detected with a thermopile in 1919 by Randall and Barker [20].

This improved description of the Mn I spectrum promptly led to the detection of doublet terms and extension of other multiplicities, so that by 1952 as compared with 1949, the total number of even levels had increased to 118 from 87, the number of odd levels to 217 from 124, and the number of classified lines to 1500 from 711 . This improvement in the term analysis of $\mathrm{Mn} \mathrm{I}$, including $g$ (magnetic splitting) factors for 108 levels, was summarized in 1952 by Charlotte E. Moore [21].

Although Back [6] in 1923 clearly demonstrated the great value of Zeeman effect in spectral term analyses, a quarter of a century elapsed before fur- 
ther determinations of inner quantum numbers and magnetic splitting factors were undertaken for Mn I.

Through the courtesy of George R. Harrison, Catalán was invited, in 1949 , to photograph the Zeeman effect in manganese spectra with the Bitter magnet and large concave gratings previously described by Harrison and Bitter [22]. The electrodes were prepared with pure manganese powder mixed with pure silver powder, then pressed and sintered to form solid rods about $3 \mathrm{~mm}$ square. These electrodes were ignited in a d-c arc operated in a magnet producing a field of 85,000 oersteds, and the spectra were photographed between $2300 \AA$ and 6500 $\AA$. These spectrograms were measured and computed partly at the National Bureau of Standards in Washington and partly at the Institute of Optics in Madrid.

In those experiments, the magnetic field intensity was determined from the splitting of the resonance lines (3280.7 and 3382.9 $\AA$ ) of the silver matrix, and/or from the resonance lines (3933.7 $\AA$ and $3968.5 \AA$ ) of ionized calcium, present as an impurity, assuming that the Zeeman patterns of those lines agree exactly with the Landé predictions [7]. Then all displacements of Zeeman components and magnetic splitting factors were expressed in Lorentz units (the unit displacement characterizing all singlet levels). As a result of these experiments, Zeeman data have been obtained for 440 lines of Mn I, ranging from $2461.0 \AA$ to $6021.8 \mathrm{~A}$, thus providing material for several reports extending over a decade.

The observations of normal Zeeman splitting in Mn I by Back [6] in 1923 were made in magnetic fields of 37,000 oersteds, whereas the Zeeman-effect spectrograms obtained by Catalán in 1949 recorded magnetic splitting in fields of 85,000 oersteds. Catalán and Velasco [23] observed changes in $g$-values of Mn I with magnetic fields above 80,000 oersteds which distort some Zeeman patterns. These distortions in the case of $z^{6} \mathrm{~F}_{01 / 2}^{\circ}$ and $z^{6} \mathrm{~F}_{1 \frac{1}{2}}^{\circ}$ levels were ascribed to repulsions between magnetic levels with equal $M$-values, belonging to adjacent levels of a spectral term, and it was shown that the theory of partial Paschen-Back effects provides a simple rule for obtaining correct $g$-values in spite of asymmetries in Zeeman patterns. This was elaborated by Catalán [24] who discussed in detail a dozen distorted patterns and concluded that the experimental $g$-values agreed with those predicted for $L S$ coupling by Landé [7]. Finally, Espinosa [25, 26], in connection with his doctoral thesis, made a complete theoretical interpretation of the very complex Paschen-Back patterns of the $z{ }^{6} \mathrm{P}^{\circ}-e{ }^{6} \mathrm{D}$ and $a{ }^{6} \mathrm{~S}-z{ }^{6} \mathrm{P}^{\circ}$ multiplets of $\mathrm{Mn} \mathrm{I}$.

The major contributions to $g$-factors for Mn I levels have been published in two papers separated by nine years. The first, by Catalán and GarciaRiquelme [27], reported measurements of Zeeman patterns for 128 lines ranging in wavelength from $2794.817 \AA$ to $4823.528 \AA$, and derived $g$-factors for 93 levels, the latter ranging in value from 0 to $67752.84 \mathrm{~K}$. The second, by Riquelme et al. [28], presented measurements and interpretations of the Zeeman patterns of 314 lines (2573 $\AA$ to $6022 \AA$ ), including derived $g$-values for 105 energy levels. The data for 251 lines were totally interpreted, whereas 63 lines showing some Paschen-Back effect were partially interpreted as confirming the spectral classification. In addition to the above, GarciaRiquelme [29] has compiled and exploited inferior Zeeman data for about 100 lines of Mn I, most of which appeared as pseudo doublets, triplets, or quartets because they were incompletely observed or resolved. In most cases, the Landé type of Zeeman pattern could be recognized and for classified lines the observed Zeeman effect confirms the classification quantitatively. Because most of the Zeeman data for Mn I have been published elsewhere $[23,24,25,26,27,28]$ they will not be repeated here; only the type numbers $[32,34]$ will be listed in our list of classified lines, and the average derived $g$-values in our table of spectral terms.

Following the new observations of wavelengths and intensities of more than 2000 spectral lines belonging to Mn I, and the measurement of Zeeman patterns for nearly 500 lines, Catalán and GarciaRiquelme undertook a complete revision of the analysis and quantum interpretation of this spectrum. A preview of this overall revision was summarized by Moore [21] in 1952 when 368 energy levels were reported to account for more than 1500 lines. This work continued practically until Catalán's death in 1957, and further progress was made by GarciaRiquelme until 1962 when the total number of accepted levels rose to 404 and classified lines exceeded 2000. At this point, several hundred unclassified lines, mostly weak, hazy, and without Zeeman data, were sent to the National Bureau of Standards where Meggers applied an electronic computer to a final search for new energy levels by adding the wave numbers of lines to the wave numbers of levels and seeking constant sums within tolerated limits. Among hundreds of "new energy levels", only two were accepted as physically real; they constitute the term $a^{2}$ I with level values of 37148.66 and $37164.25 \mathrm{~K}$. We conclude that the available material on Mn I does not permit further progress in the analysis of this spectrum. This conclusion and the fact that our material is more homogeneous and extensive than any existing description of Mn I justify publication of this paper.

\section{Results}

Our final results for $\mathrm{Mn}$ I classified lines are presented in table 1 , in which col. 1 contains the observed wavelength $(\lambda)$ in angstroms $(\AA)$; col. 2 , the intensity and character; col. 3 , the wave number $(\sigma)$ in kaysers $(\mathrm{K})$; col. 4, the observed minus calculated wave number in kaysers $(\mathrm{K})$; col. 5, the symbols for associated energy levels; and col. 6, the Zeeman type. Since the "Air" wavelengths were measured before 1950 , they were converted to vacuum wave numbers with the aid of Kayser's Table [30] which was recently superseded by a new table published by Coleman, Bozman, and Meggers [31]. Because 
Table 1. Mn I-Classified lines

\begin{tabular}{|c|c|c|c|c|c|}
\hline 1 & 2 & 3 & 4 & 5 & 6 \\
\hline$\lambda$ & Int. & $\sigma$ & $\mathrm{o}-\mathrm{c}$ & Term designation & $\begin{array}{l}\text { Zeeman } \\
\text { type }\end{array}$ \\
\hline $\begin{array}{c}\AA \\
\text { Vac } \\
1785.355 \\
1785.465 \\
1785.829 \\
1788.152 \\
1875.727\end{array}$ & $\begin{array}{r}7 h \\
5 h \\
6 h \\
2 h \\
10 r\end{array}$ & $\begin{array}{c}K \\
56011.26 \\
56007.81 \\
55996.40 \\
55923.65 \\
53312.66\end{array}$ & $\begin{array}{r}K \\
-1.16 \\
-0.10 \\
-0.22 \\
-0.16 \\
1.29\end{array}$ & $\begin{array}{l}a{ }^{6} \mathrm{~S}_{21 / 2}-s{ }^{6} \mathrm{P}_{31 / 2}^{\circ} \\
a^{6} \mathrm{~S}_{21 / 2}-s{ }^{6} \mathrm{P}_{21 / 2}^{o} \\
a^{6} \mathrm{~S}_{21 / 2}-s{ }^{6} \mathrm{P}_{11 / 2}^{\circ} \\
a^{6} \mathrm{~S}_{21 / 2}-55923^{\circ}{ }_{21 / 2} \\
a^{6} \mathrm{~S}_{21 / 2}-t^{6} \mathrm{P}_{11 / 2}^{\circ}\end{array}$ & \\
\hline $\begin{array}{l}\text { 1876. } 445 \\
1877.518 \\
1882.366 \\
1882.900 \\
1883.085\end{array}$ & $\begin{array}{c}15 r \\
20 r \\
3 \\
1 \\
0\end{array}$ & $\begin{array}{l}53292.26 \\
53261.80 \\
53124.63 \\
53109.56 \\
53104.34\end{array}$ & $\begin{array}{l}0.68 \\
0.38 \\
0.54 \\
0.35 \\
1.15\end{array}$ & $\begin{array}{l}a^{6} \mathrm{~S}_{21 / 2}-t^{6} \mathrm{P}_{21 / 2}^{\circ} \\
a^{6} \mathrm{~S}_{21 / 2}-t^{6} \mathrm{P}_{31 / 2}^{\circ} \\
a^{6} \mathrm{~S}_{21 / 2}-y^{4} \mathrm{D}_{31 / 2}^{\circ} \\
a^{6} \mathrm{~S}_{21 / 2}-y^{4} \mathrm{D}_{21 / 2}^{0} \\
a^{6} \mathrm{~S}_{21 / 2}-y^{4} \mathrm{D}_{11 / 2}^{\circ}\end{array}$ & \\
\hline $\begin{array}{l}\text { 1890. } 962 \\
1891.414 \\
1913.752 \\
1918.328 \\
1922.516\end{array}$ & $\begin{array}{c}1 \\
3 \\
(15) \\
(15) \\
(12)\end{array}$ & $\begin{array}{l}52883.13 \\
52870.49 \\
52253.57 \\
52128.73 \\
52015.17\end{array}$ & $\begin{array}{r}-0.74 \\
0.39 \\
0.33 \\
0.08 \\
0.17\end{array}$ & 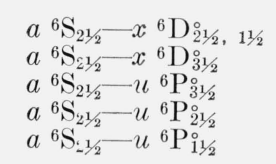 & \\
\hline $\begin{array}{l}1949.100 \\
1996.056 \\
1999.511\end{array}$ & $\begin{array}{c}3 \\
50 \mathrm{R} \\
100 \mathrm{R}\end{array}$ & $\begin{array}{l}51305.73 \\
50098.79 \\
50012.23\end{array}$ & $\begin{array}{r}0.32 \\
-0.37 \\
-0.30\end{array}$ & $\begin{array}{l}a{ }^{6} \mathrm{~S}_{21 / 2}-x \\
a^{6} \mathrm{~S}_{21 / 2} \mathrm{P}^{6} \mathrm{P}^{\circ}{ }^{6} \mathrm{P}_{11 / 2} \\
a^{6} \mathrm{~S}_{21 / 2}-v \\
{ }^{6} \mathrm{P}_{21 / 2}^{\circ}\end{array}$ & \\
\hline Air & & & & & \\
\hline $\begin{array}{l}2003.849 \\
2017.630\end{array}$ & $\begin{array}{c}200 \mathrm{R} \\
3\end{array}$ & $\begin{array}{l}\text { 49887. } 73 \\
49547.04\end{array}$ & $\begin{array}{l}-0.35 \\
-0.84\end{array}$ & $\begin{array}{c}a^{6} \mathrm{~S}_{21 / 2}-v{ }^{6} \mathrm{P}_{31 / 2}^{\circ} \\
a^{6} \mathrm{D}_{41 / 2}-66600_{31 / 2}^{\circ} ?\end{array}$ & \\
\hline $\begin{array}{l}\text { 2018. } 332 \\
2070.988 \\
2072.917 \\
2092.159 \\
2092.516\end{array}$ & $\begin{array}{c}4 \\
5 \\
12 \\
500 \mathrm{R} \\
50 \mathrm{R}\end{array}$ & $\begin{array}{l}49529.78 \\
48270.66 \\
48225.74 \\
47782.26 \\
47774.09\end{array}$ & $\begin{array}{r}0.00 \\
-0.25 \\
-0.25 \\
-0.17 \\
-0.43\end{array}$ & 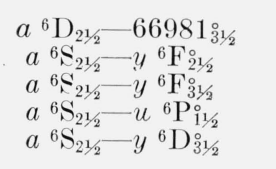 & \\
\hline $\begin{array}{l}\text { 2093. } 407 \\
2097.554 \\
2106.052 \\
2109.585 \\
2173.195\end{array}$ & $\begin{array}{c}200 \mathrm{R} \\
30 \\
100 \mathrm{R} \\
300 \mathrm{R} \\
3 \mathrm{~h}\end{array}$ & $\begin{array}{l}47753.77 \\
47659.38 \\
47467.10 \\
47387.60 \\
46001.72\end{array}$ & $\begin{array}{r}-0.22 \\
-0.14 \\
0.44 \\
-0.02 \\
0.95\end{array}$ & $\begin{array}{l}a^{6} \mathrm{~S}_{21 / 2}-y{ }^{6} \mathrm{D}_{21 / 2}^{\circ} \\
a^{6} \mathrm{~S}_{21 / 2}-w^{6} \mathrm{P}_{21 / 2}^{0} \\
a^{6} \mathrm{~S}_{21 / 2}-y{ }^{6} \mathrm{D}_{11 / 2}^{0} \\
a^{6} \mathrm{~S}_{21 / 2}-w^{6} \mathrm{P}_{31 / 2}^{o} \\
a^{6} \mathrm{~S}_{21 / 2}-y{ }^{8} \mathrm{P}_{31 / 2}^{011}\end{array}$ & \\
\hline $\begin{array}{l}\text { 2174. } 12 \\
2176.014 \\
2182.773 \\
2184.912 \\
2190.884\end{array}$ & $\begin{array}{c}0 h \\
2 \\
15 \\
10 \\
2\end{array}$ & $\begin{array}{l}\text { 45981. } 2 \\
45941.14 \\
45798.90 \\
45754.07 \\
45629.37\end{array}$ & $\begin{array}{r}-0.2 \\
0.21 \\
-0.28 \\
-0.20 \\
-0.39\end{array}$ & $\begin{array}{c}a{ }^{6} \mathrm{~S}_{21 / 2}-y{ }^{8} \mathrm{P}_{21 / 2}^{0}{ }^{2} \\
a{ }^{6} \mathrm{~S}_{21 / 2}-z^{4} \mathrm{D}_{21 / 2}^{\circ} \\
a^{6} \mathrm{D}_{41 / 2}-w^{6} \mathrm{D}_{31 / 2}^{0}{ }^{1}{ }^{1} \\
a{ }^{6} \mathrm{~S}_{21 / 2}-z^{4} \mathrm{D}_{31 / 2}^{0} \\
a^{6} \mathrm{D}_{21 / 2}-y^{2} \mathrm{D}_{21 / 2}^{\circ}\end{array}$ & \\
\hline $\begin{array}{l}\text { 2191. } 413 \\
\text { 2193. } 762 \\
2196.503 \\
\text { 2198. } 131 \\
\text { 2199. } 41\end{array}$ & $\begin{array}{r}100 \\
20 \\
3 \\
10 \\
1 h\end{array}$ & $\begin{array}{l}45618.36 \\
45569.51 \\
45512.65 \\
45478.94 \\
45452.5\end{array}$ & $\begin{array}{r}-0.16 \\
0.04 \\
-0.15 \\
-0.39 \\
-0.5\end{array}$ & $\begin{array}{l}a^{6} \mathrm{D}_{41 / 2}-w^{6} \mathrm{D}_{41 / 2}^{\circ} \\
a^{6} \mathrm{D}_{31 / 2}-w^{6} \mathrm{D}_{31 / 2}^{\circ} \\
a^{6} \mathrm{D}_{11 / 2}-y^{2} \mathrm{D}_{21 / 2}^{\circ} \\
a^{6} \mathrm{D}_{31 / 2}-w^{6} \mathrm{D}_{21 / 2}^{\circ} \\
a^{6} \mathrm{D}_{41 / 2}-v^{4} \mathrm{~F}_{31 / 2}^{\circ}\end{array}$ & \\
\hline $\begin{array}{l}\text { 2201. } 960 \\
2202.489 \\
\text { 2205. } 057 \\
2206.343 \\
2208.806\end{array}$ & $\begin{array}{c}7 \\
2 \\
10 \\
2 \\
200 \mathrm{R}\end{array}$ & $\begin{array}{l}45399.88 \\
45388.97 \\
45336.11 \\
45309.70 \\
45259.17\end{array}$ & $\begin{array}{r}-0.07 \\
0.16 \\
0.00 \\
-0.11 \\
0.00\end{array}$ & $\begin{array}{c}a^{6}{ }^{6} \mathrm{D}_{21 / 2}-w^{6} \mathrm{D}_{31 / 2}^{0} \\
a^{6} \mathrm{D}_{31 / 2}-w^{6} \mathrm{D}_{41 / 2}^{31} \\
a^{6} \mathrm{D}_{21 / 2}-w^{6} \mathrm{D}_{11 / 2}^{\circ} \\
a^{6} \mathrm{D}_{21 / 2}-w^{6} \mathrm{D}_{21 / 2}^{\circ} \\
a^{6} \mathrm{~S}_{21 / 2}-x^{6} \mathrm{P}_{11 / 2}^{\circ}\end{array}$ & \\
\hline $\begin{array}{l}2210.582 \\
2211.720 \\
2212.055 \\
2213.855 \\
2214.10\end{array}$ & $\begin{array}{c}8 h \\
8 \\
15 \\
300 \mathrm{R} \\
10\end{array}$ & $\begin{array}{l}45222.81 \\
45199.55 \\
45192.70 \\
45155.96 \\
45150.4\end{array}$ & $\begin{array}{l}-0.48 \\
-0.13 \\
-0.15 \\
-0.15 \\
-0.1\end{array}$ & $\begin{array}{c}a^{6} \mathrm{D}_{31 / 2}-v^{4} \mathrm{~F}_{31 / 2}^{0} \\
a^{6} \mathrm{D}_{11 / 2}-w^{6} \mathrm{D}_{01 / 2}^{2} \\
a^{6} \mathrm{D}_{11 / 2}-w^{6} \mathrm{D}_{21 / 2}^{\circ} \\
a{ }^{6} \mathrm{~S}_{21 / 2}-x{ }^{6} \mathrm{P}_{21 / 2}^{0} \\
a^{6} \mathrm{D}_{01 / 2}-w^{6} \mathrm{D}_{11 / 2}^{2}\end{array}$ & \\
\hline $\begin{array}{l}\text { 2215. } 086 \\
2218.903 \\
2221.837 \\
2249.911 \\
2258.714\end{array}$ & $\begin{array}{c}3 \\
3 h \\
500 \mathrm{R} \\
5 s \\
2\end{array}$ & $\begin{array}{l}45130.89 \\
45053.24 \\
44993.76 \\
44432.39 \\
44259.24\end{array}$ & $\begin{array}{l}-0.12 \\
-0.53 \\
-0.16 \\
-0.13 \\
-0.22\end{array}$ & $\begin{array}{c}a{ }^{6} \mathrm{D}_{01 / 2}-w^{6} \mathrm{D}_{01 / 2}^{\circ} \\
a^{6} \mathrm{D}_{21 / 2}-v^{4} \mathrm{~F}_{01 / 2}^{\circ} \\
a{ }^{6} \mathrm{~S}_{21 / 2}-x \\
{ }^{6} \mathrm{P}_{31 / 2}^{\circ} \\
a^{6} \mathrm{D}_{31 / 2}-y^{2} \mathrm{G}_{41 / 2}^{\circ} \\
a^{6} \mathrm{D}_{21 / 2}-z^{2} \mathrm{~F}_{31 / 2}^{0}\end{array}$ & \\
\hline
\end{tabular}


TABle 1. Mn I-Classified lines-Continued

\begin{tabular}{|c|c|c|c|c|c|}
\hline 1 & 2 & 3 & 4 & 5 & 6 \\
\hline$\lambda$ & Int. & $\sigma$ & $o-c$ & Term designation & $\begin{array}{l}\text { Zeeman } \\
\text { type }\end{array}$ \\
\hline$\underset{\mathrm{Air}}{\stackrel{̊}{\mathrm{~A}}}$ & & $K$ & $K$ & & \\
\hline 2262. 294 & $8 s$ & 44189. 21 & -0.02 & $a^{6} \mathrm{D}_{31 / 2}-w^{4} \mathrm{G}_{21 / 2}^{\circ}$ & \\
\hline 2277.065 & $0 h$ & 43902.60 & -0.15 & $a^{6} \mathrm{D}_{11 / 2}-w^{4} \mathrm{G}_{21 / 2}^{20 / 2}$ & \\
\hline 2288. 449 & 20 & 43684. 22 & -0.41 & $a^{4} \mathrm{D}_{31 / 2}^{172}-66981_{31 / 2}^{\circ}$ & \\
\hline 2292. 189 & 30 & 43612.95 & -0.40 & $a^{4} \mathrm{D}_{31 / 2}^{1 / 2}-66910_{21 / 2}^{2}$ & \\
\hline 2293. 122 & 2 & 43595. 21 & -0.29 & $a{ }^{6} \mathrm{~S}_{21 / 2}-z^{6} \mathrm{~F}_{21 / 2}^{o}$ & \\
\hline 2296. 880 & 5 & 43523. 88 & -0.20 & $a^{6} \mathrm{~S}_{21 / 2}-z^{6} \mathrm{~F}_{31 / 2}^{\circ}$ & \\
\hline 2298. 876 & 20 & 43486. 10 & -0.28 & $a{ }^{4} \mathrm{D}_{31 / 2}-u{ }^{4} \mathrm{~F}_{31 / 2}^{\circ}$ & \\
\hline 2300. 300 & $\begin{array}{l}8 \\
3 \mathrm{H}\end{array}$ & $\begin{array}{l}43459.18 \\
43451.10\end{array}$ & $\begin{array}{l}-0.16 \\
-0.18\end{array}$ & $a^{+} \mathrm{D}_{21 / 2}-67008_{21 / 2}^{\circ}$ & \\
\hline $\begin{array}{l}\text { 2300. } 728 \\
2301.260\end{array}$ & $1 d$ & $\begin{array}{l}\text { 43451. } 10 \\
43441.07\end{array}$ & $\begin{array}{l}-0.18 \\
-0.08\end{array}$ & $\begin{array}{l}a^{6} \mathrm{D}_{21 / 2}-w^{4} \mathrm{~F}_{31 / 2}^{1} \\
a^{4} \mathrm{D}_{31 / 2}-v^{2} \mathrm{G}_{31 / 2}^{0}\end{array}$ & \\
\hline 2301. 748 & 4 & 43431. 84 & -0.26 & $a{ }^{4} \mathrm{D}_{21 / 2}-66981_{31 / 2}^{\circ}$ & \\
\hline 2305.518 & 5 & 43360.83 & 0.01 & $a^{4} \mathrm{D}_{21 / 2}-66910_{21 / 2}^{\circ}$ & \\
\hline 2305. 703 & $15 \mathrm{H}$ & 43357. 35 & -0.63 & $a{ }^{4} \mathrm{D}_{31 / 2}-66654_{21 / 2}$ & \\
\hline 2309. 057 & $2 h$ & 43294. 38 & -0.21 & $a^{4} \mathrm{D}_{21 / 2}-u^{4} \mathrm{~F}_{11 / 2}^{0}$ & \\
\hline 2309. 374 & 10 & 43288. 45 & 0.01 & $a^{4} \mathrm{D}_{21 / 2}-u^{4} \mathrm{~F}_{21 / 2}^{\circ}$ & \\
\hline 2312. 304 & 20 & 43233. 59 & -0.26 & $a{ }^{4} \mathrm{D}_{21 / 2}-u{ }^{4} \mathrm{~F}_{31 / 2}^{\circ}$ & \\
\hline 2318.17 & $1 h$ & 43124. 2 & -0.1 & $a^{4} \mathrm{D}_{11 / 2}-u{ }^{4} \mathrm{~F}_{11 / 2}$ & \\
\hline $\begin{array}{l}2318.501 \\
2321.995\end{array}$ & $\begin{array}{r}2 h \\
10 h\end{array}$ & 43118. 04 & $\begin{array}{r}-0.08 \\
-0.00\end{array}$ & $\begin{array}{l}a^{4} \mathrm{D}_{11 / 2}-u{ }^{4} \mathrm{~F}_{21 / 2}^{\circ} \\
a^{4} \mathrm{G}-u{ }^{2} \mathrm{G}^{\circ}\end{array}$ & \\
\hline 2321. 995 & & 43053. 16 & $\begin{array}{l}0.00 \\
0.26\end{array}$ & $\begin{array}{l}a^{4} \mathrm{G}_{41 / 2}-u^{2} \mathrm{G}_{31 / 2}^{0} \\
a^{4} \mathrm{G}_{31 / 2}-u^{2} \mathrm{G}_{31 / 2}^{0}\end{array}$ & \\
\hline 2322. 106 & $3 h$ & 43051. 11 & 0.14 & $a^{4} \mathrm{D}_{21 / 2}-66600_{31 / 2}^{2}$ & \\
\hline 2323. 748 & $30 h$ & 43020. 70 & 0.00 & $a^{4} \mathrm{G}_{51 / 2}-u^{2} \mathrm{G}_{41 / 2}^{0}$ & \\
\hline 2324.803 & $5 h$ & 43001. 17 & 0.16 & $a^{4} \mathrm{G}_{41 / 2}-u^{2} \mathrm{G}_{41 / 2}^{1 / 2}$ & \\
\hline 2327. 308 & $2 \mathrm{H}$ & 42954. 89 & -0.12 & $a^{4} \mathrm{D}_{21 / 2}^{1 / 2}-66504_{11 / 2}^{2}$ & \\
\hline 2342. 088 & $3 \mathrm{H}$ & 42683. 85 & 0. 02 & $a{ }^{4} \mathrm{G}_{21 / 2}-t^{4} \mathrm{G}_{21 / 2}^{\circ}$ & \\
\hline 2342. 771 & $1 \mathrm{H}$ & 42676.87 & -0.26 & $a{ }^{4} \mathrm{G}_{31 / 2}-t^{4} \mathrm{G}_{21 / 2}^{0}$ & \\
\hline 2346. 126 & $1 \mathrm{H}$ & 42610. 38 & 0. 06 & $a^{4} \mathrm{G}_{21 / 2}-t^{4} \mathrm{G}_{31 / 2}^{0}$ & \\
\hline 2346. 383 & $2 h$ & 42605. 72 & -0.21 & $a^{4} \mathrm{G}_{41 / 2}-t^{4} \mathrm{G}_{31 / 2}^{0}$ & \\
\hline $\begin{array}{l}2346.497 \\
2349.263\end{array}$ & $\begin{array}{l}5 h \\
3 h\end{array}$ & 42603. 65 & $\begin{array}{l}0.03 \\
0.07\end{array}$ & $a^{4} \mathrm{G}_{31 / 2}-t^{4} \mathrm{G}_{31 / 2}^{0}$ & \\
\hline $\begin{array}{l}\text { 2349. } 263 \\
2350.352\end{array}$ & $10 h$ & $\begin{array}{l}42553.50 \\
42533.78\end{array}$ & 0.04 & $\begin{array}{l}a^{4} \mathrm{G}_{51 / 2}-t^{4} \mathrm{G}_{41 / 2}^{01} \\
a^{4} \mathrm{G}_{41 / 2}-t^{4} \mathrm{G}_{41 / 2}^{\circ}\end{array}$ & \\
\hline 2352. 937 & $20 h$ & 42487. 05 & -0.05 & $a^{4} \mathrm{G}_{51 / 2}-t^{4} \mathrm{G}_{51 / 2}^{\circ}$ & \\
\hline 2354. 020 & $4 h$ & 42467. 51 & 0.10 & $a^{4} \mathrm{G}_{41 / 2}-t^{4} \mathrm{G}_{51 / 2}^{\circ}$ & \\
\hline 2357. 899 & $2 h$ & 42397. 65 & -0.02 & $a^{4} \mathrm{D}_{21 / 2}-w^{2} \mathrm{D}_{21 / 2}^{2}$ & \\
\hline 2362. 719 & 1 & 42311. 17 & $\begin{array}{l}0.07 \\
0.00\end{array}$ & $a^{4} \mathrm{G}_{51 / 2}-w^{2} \mathrm{H}_{41 / 2}^{\circ}$ & \\
\hline 2363. 823 & 4 & 42291.41 & 0.00 & $a^{4} \mathrm{G}_{41 / 2}-w^{2} \mathrm{H}_{41 / 2}^{\circ}$ & \\
\hline 2363. 956 & 1 & 42289. 03 & -0.07 & $a^{4} \mathrm{G}_{31 / 2}-w^{2} \mathrm{H}_{41 / 2}^{\circ}$ & \\
\hline 2366. 575 & 1 & 42242. 24 & -0.14 & $a^{4} \mathrm{D}_{11 / 2}-w^{2} \mathrm{D}_{11 / 2}^{\circ}$ & \\
\hline 2366. 744 & 5 & 42239. 22 & 0. 06 & $a^{4} \mathrm{G}_{51 / 2}-w^{2} \mathrm{H}_{51 / 2}^{0}$ & \\
\hline $\begin{array}{l}\text { 2367. } 851 \\
2372.116\end{array}$ & $\begin{array}{c}1 \\
10 d\end{array}$ & $\begin{array}{l}42219.47 \\
42143.56\end{array}$ & $\begin{array}{r}0.00 \\
-0.01\end{array}$ & $\begin{array}{c}a^{4} \mathrm{G}_{11 / 2}-w^{2} \mathrm{H}_{51 / 2}^{\circ} \\
a^{6} \mathrm{~S}_{21 / 2}-z^{6} \mathrm{D}_{11 / 2}^{\circ}\end{array}$ & \\
\hline 2377. 183 & $30 \mathrm{R}$ & 42053. 75 & 0.02 & $a^{6} \mathrm{~S}_{21 / 2}-z^{6} \mathrm{D}_{21 / 2}^{\circ}$ & \\
\hline 2382.175 & 2 & 41965. 63 & 0.02 & $a^{4} \mathrm{D}_{31 / 2}-w^{2} \mathrm{G}_{41 / 2}^{\circ / 2}$ & \\
\hline 2384. 049 & $40 \mathrm{R}$ & 41932. 65 & 0.01 & $a^{6} \mathrm{~S}_{21 / 2}-z^{6} \mathrm{D}_{31 / 2}^{0}$ & \\
\hline 2397. 732 & 2 & 41693. 37 & -0.19 & $a{ }^{4} \mathrm{G}_{31 / 2}-66981_{31 / 2}^{0}$ & \\
\hline 2401. 830 & 2 & 41622. 24 & -0.04 & $a{ }^{4} \mathrm{G}_{31 / 2}-66910_{21 / 2}^{\circ}$ & \\
\hline 2403. 748 & 2 & 41589. 03 & -0.23 & $a^{4} \mathrm{G}_{51 / 2}-u^{4} \mathrm{~F}_{41 / 2}^{\circ}$ & \\
\hline 2404. 882 & 5 & 41569. 42 & -0.15 & $a^{4} \mathrm{G}_{41 / 2}-u^{4} \mathrm{~F}_{41 / 2}$ & \\
\hline 2405. 274 & $\begin{array}{l}3 \\
2 h\end{array}$ & $\begin{array}{l}41562.65 \\
41556.53\end{array}$ & -0.10 & $a^{4} \mathrm{G}_{21 / 2}-u^{4} \mathrm{~F}_{11 / 2}$ & \\
\hline $\begin{array}{l}2405.628 \\
2406.017\end{array}$ & $\begin{array}{l}2 h \\
2\end{array}$ & $\begin{array}{l}41556.53 \\
41549.82\end{array}$ & $\begin{array}{l}-0.07 \\
-0.08\end{array}$ & $\begin{array}{l}a^{4} \mathrm{G}_{21 / 2}-u^{4} \mathrm{~F}_{21 / 2}^{1} \\
a^{4} \mathrm{G}_{31 / 2}-u^{4} \mathrm{~F}_{21 / 2}^{2}\end{array}$ & \\
\hline 2409. 198 & 2 & 41494. 96 & -0.35 & $a^{4} \mathrm{G}_{31 / 2}-u^{4} \mathrm{~F}_{31 / 2}^{0}$ & \\
\hline 2411. 415 & 2 & 41456.81 & 0. 03 & $a^{4} \mathrm{G}_{21 / 2}-v^{2} \mathrm{G}_{31 / 2}^{0}$ & \\
\hline 2417. 502 & 2 & 41352. 44 & -0.09 & $a^{4} \mathrm{D}_{31 / 2}-x^{2} \mathrm{G}_{31 / 2}$ & \\
\hline $\begin{array}{l}2417.909 \\
2418.049\end{array}$ & 2 & 41345. 48 & -0.01 & $a^{4} \mathrm{G}_{41 / 2}-v^{2} \mathrm{G}_{41 / 2}^{\circ}$ & \\
\hline 2418.042 & 4 & 41343. 20 & 0.02 & $a^{4} \mathrm{G}_{31 / 2}-v^{2} \mathrm{G}_{41 / 2}^{\circ}$ & \\
\hline
\end{tabular}


Table 1. Mn I-Classified lines-Continued

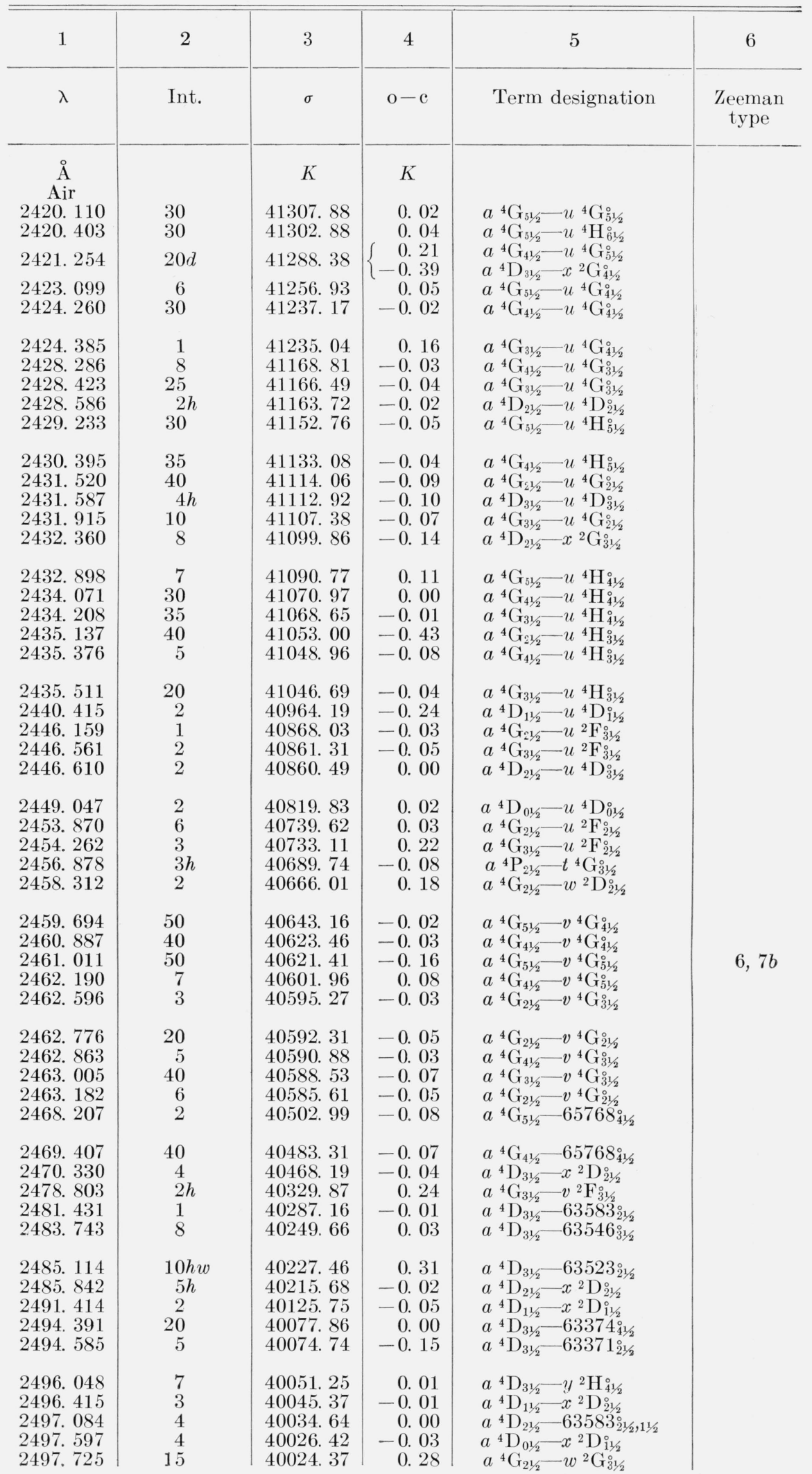


Table 1. Mn I-Classified lines-Continued

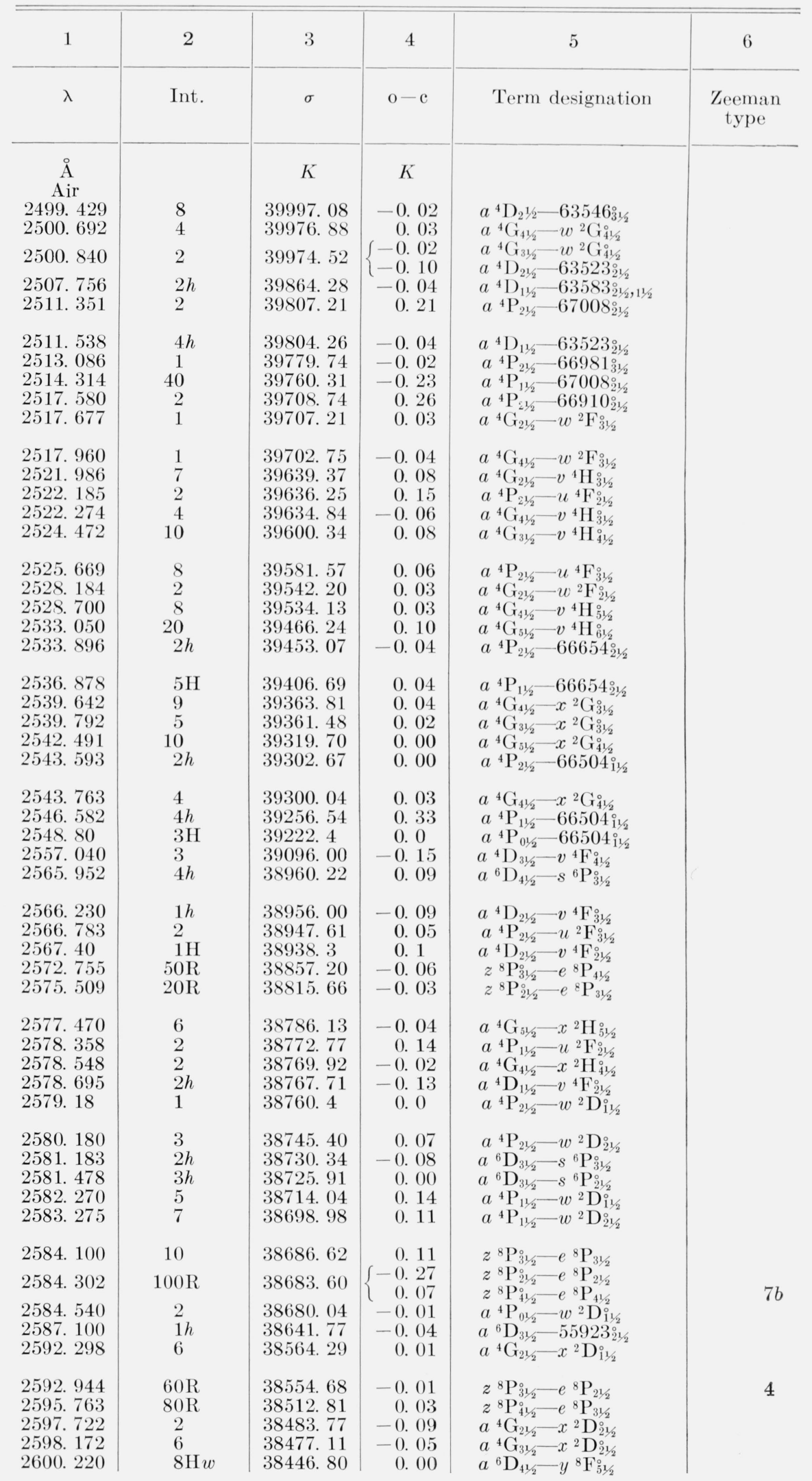


TaBle 1. Mn I-Classified lines-Continued

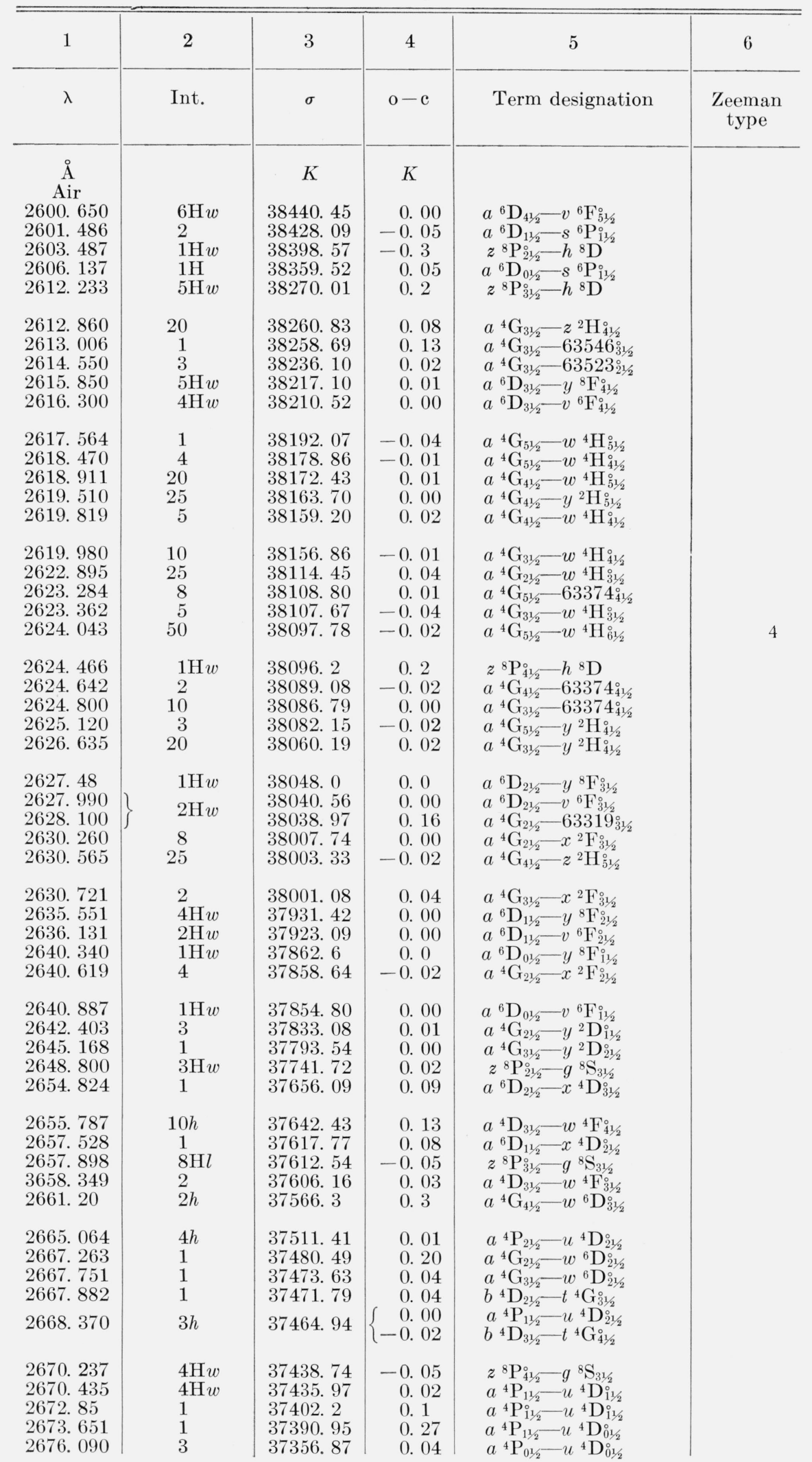


TABle 1. Mn I-Classified lines-Continued

\begin{tabular}{|c|c|c|c|c|c|}
\hline 1 & 2 & 3 & 4 & 5 & 6 \\
\hline$\lambda$ & Int. & $\sigma$ & $\mathrm{o}-\mathrm{c}$ & Term designation & $\begin{array}{l}\text { Zeeman } \\
\text { type }\end{array}$ \\
\hline $\begin{array}{c}\AA \\
\text { Air }\end{array}$ & & $K$ & $K$ & & \\
\hline 2676. 326 & 10 & 37353.57 & -0.03 & $a^{4} \mathrm{D}_{21 / 2}-w^{4} \mathrm{~F}_{31 / 2}^{0}$ & \\
\hline 2682. 244 & 2 & 37271. 16 & 0.01 & $a^{4} \mathrm{D}_{21 / 2}-w^{4} \mathrm{~F}_{21 / 2}^{0}$ & \\
\hline 2685. 941 & $8 \mathrm{H}$ & 37219.86 & 0.00 & $a^{4} \mathrm{G}_{41 / 2}-v{ }^{4} \mathrm{~F}_{31 / 2}^{\circ}$ & \\
\hline $\begin{array}{l}2686.777 \\
2687.400\end{array}$ & $\begin{array}{l}6 \\
8 h\end{array}$ & $\begin{array}{l}37208.28 \\
37199.66\end{array}$ & $\begin{array}{l}0.13 \\
0.04\end{array}$ & $\begin{array}{l}a^{4} \mathrm{P}_{21 / 2}-u^{4} \mathrm{D}_{31 / 2} \\
a^{4} \mathrm{G}_{31}-v^{4} \mathrm{~F}_{21 / 2}^{0}\end{array}$ & \\
\hline 2688.078 & 2 & 37190.28 & 0.06 & $a^{4} \mathrm{D}_{21}-z^{2} \mathrm{G}_{21}$ & \\
\hline 2690. 977 & 2 & 37150.21 & -0.05 & $z^{8} \mathrm{P}_{31 / 2}^{21 / 2}-h^{6} \mathrm{D}_{41 / 2}$ & \\
\hline 2692. 655 & 20 & 37127. 06 & -0.02 & $a^{4} \mathrm{G}_{51 / 2}^{0}-v^{4} \mathrm{~F}_{41 / 2}^{0}$ & \\
\hline 2693. 757 & 2 & 37109.12 & -0.04 & $a^{4} \mathrm{G}_{21 / 2}-v^{4} \mathrm{~F}_{11 / 2}^{0}$ & \\
\hline 2694. 560 & & 37100.82 & -0.01 & $a^{4} \mathrm{D}_{11 / 2}-w^{4} \mathrm{~F}_{21 / 2}^{2}$ & \\
\hline 2698. 890 & 2 & 37041.30 & -0.05 & $a^{4} \mathrm{D}_{11 / 2}-w^{4} \mathrm{~F}_{11 / 2}^{0}$ & \\
\hline 2703. 129 & $20 \mathrm{H} w$ & 36983. 21 & 0.48 & $z^{8} \mathrm{P}_{41 / 2}^{0}-h^{6} \mathrm{D}_{31 / 2}^{1}$ & \\
\hline 2703. 658 & $50 \mathrm{H} w$ & 36975. 96 & -0.57 & $z^{8} \mathrm{P}_{41 / 2}^{0}-h^{6} \mathrm{D}_{41 / 2}$ & \\
\hline $\begin{array}{l}2703.92 \\
2706.142\end{array}$ & $\begin{array}{l}40 \mathrm{H} w \\
5\end{array}$ & $\begin{array}{l}36972.4 \\
36942.04\end{array}$ & $\begin{array}{l}0.1 \\
0.04\end{array}$ & $\begin{array}{c}z^{8} \mathrm{P}_{21 / 2}-g^{8} \mathrm{D}_{31 / 2} \\
a^{4} \mathrm{D}_{01 / 2}-w^{4} \mathrm{~F}_{11 / 2}\end{array}$ & \\
\hline 2713. 320 & $100 \mathrm{H} w$ & 36844. 31 & 0.25 & $z^{8} \mathrm{P}_{31 / 2}^{0}-g^{8} \mathrm{D}_{41 / 2}$ & \\
\hline 2717.028 & & 36794. 04 & 0.06 & $a^{4} \mathrm{G}_{21 / 2}-y^{2} \mathrm{~F}_{21 / 2}$ & \\
\hline 2720. 387 & $2 h$ & 36748.61 & 0.00 & $a^{4} \mathrm{G}_{41 / 2}-y^{2} \mathrm{~F}_{31 / 2}^{1}$ & \\
\hline 2720.550 & $5 h$ & 36746.41 & 0. 11 & $a^{4} \mathrm{G}_{31 / 2}-y^{2} \mathrm{~F}_{31 / 2}$ & \\
\hline 2726.13 & $100 \mathrm{H} u$ & 36671.2 & -0.1 & $z^{8} \mathrm{P}_{41 / 2}^{o}-g^{8} \mathrm{D}_{51 / 2}$ & \\
\hline 2727. 381 & 1 & 36654. 37 & 0.04 & $b{ }^{4} \mathrm{D}_{31 / 2}-67008_{21 / 2}^{\circ}$ & \\
\hline 2729. 420 & 10 & 36627.00 & -0.09 & $b^{4} \mathrm{D}_{31 / 2}-66981_{31 / 2}^{\circ}$ & \\
\hline 2732. 260 & 2 & $\begin{array}{l}36588.93 \\
36576.78\end{array}$ & 0. 00 & $b^{4} \mathrm{D}_{21 / 2}-67008^{21 / 2}$ & \\
\hline 2733. 167 & 1 & 36576.78 & 0. 01 & $\begin{array}{l}a^{4} \mathrm{D}_{01 / 2}-z^{2} \mathrm{D}_{1 / 2}^{\circ} \\
a^{4} \mathrm{P}^{2} x^{2} \mathrm{D}_{0}^{\circ}\end{array}$ & \\
\hline 2734. 164 & 1 & 36563.45 & $\left\{\begin{array}{r}0.09 \\
-0.02\end{array}\right.$ & $\begin{array}{l}a^{4} \mathrm{P}_{21 / 2}-x^{2} \mathrm{D}_{21 / 2}^{\circ} \\
a^{4} \mathrm{P}_{01 / 2}-x^{2} \mathrm{D}_{11 / 2}^{\circ}\end{array}$ & \\
\hline 2734. 297 & 1 & 36561.67 & -0.02 & $b^{4} \mathrm{D}_{21 / 2}-66981_{31 / 2}^{\circ}$ & \\
\hline 2734. 736 & 15 & 36555.80 & -0.01 & $b^{4} \mathrm{D}_{31 / 2}-66910_{21 / 2}^{\circ}$ & \\
\hline 2734. 997 & 4 & 36552.31 & -0.14 & $a{ }^{4} \mathrm{D}_{21 / 2}-z^{2} \mathrm{D}_{21 / 2}^{1 / 2}$ & \\
\hline 2735. 310 & $1 h w$ & 36548.13 & -0.07 & $z^{8} \mathrm{P}_{21 / 2}^{0}-g^{6} \mathrm{D}_{21 / 2}$ & \\
\hline 2737. 640 & & 36517.03 & 0.13 & $a^{4} \mathrm{P}_{11 / 2}-x^{2} \mathrm{D}_{21 / 2}$ & \\
\hline 2738. 552 & 5 & 36504. 87 & -0.03 & $a^{4} \mathrm{G}_{21 / 2}-y^{2} \mathrm{G}_{31 / 2}^{\circ}$ & \\
\hline 2738. 861 & 25 & 36500.75 & -0.04 & $b{ }^{4} \mathrm{D}_{31 / 2}-u{ }^{4} \mathrm{~F}_{41 / 2}$ & \\
\hline 2739. 627 & 4 & 36490.54 & 0. 13 & & \\
\hline $\begin{array}{l}2740.161 \\
2740.546\end{array}$ & $\begin{array}{l}1 h \\
3 \mathrm{H} w\end{array}$ & $\begin{array}{l}36483.43 \\
36478.31\end{array}$ & $\begin{array}{l}0.00 \\
0.01\end{array}$ & $\begin{array}{l}b{ }^{4} \mathrm{D}_{31 / 2}-u{ }^{4} \mathrm{~F}_{21 / 2}^{\circ} \\
a^{4} \mathrm{G}_{51 / 2}-61744_{51 / 2}^{\circ}\end{array}$ & \\
\hline 2742. 735 & 15 & 36449. 19 & 0.41 & $a^{4} \mathrm{G}_{51 / 2}-y^{2} \mathrm{G}_{41 / 2}^{\circ} ?$ & \\
\hline 2742. 930 & 1 & 36446. 60 & 0.18 & $a^{4} \mathrm{G}_{21 / 2}-z^{2} \mathrm{~F}_{21 / 2}^{2}$ & \\
\hline 2743. 446 & 10 & 36439.75 & 0.03 & $a^{4} \mathrm{G}_{31 / 2}-z^{2} \mathrm{~F}_{21 / 2}^{0}$ & \\
\hline 2743. 790 & 5 & 36435.18 & - 0.09 & $a^{4} \mathrm{D}_{31 / 2}-x^{4} \mathrm{G}_{41 / 2}$ & \\
\hline 2744.023 & & 36432. 08 & 0.03 & $b{ }^{4} \mathrm{D}_{01 / 2}-u{ }^{4} \mathrm{~F}_{11 / 2}^{0}$ & \\
\hline 2744. 268 & $6 h$ & 36428.84 & $\left\{\begin{array}{r}-0.25 \\
0.00\end{array}\right.$ & $\begin{array}{l}a^{4} \mathrm{G}_{41 / 2}-y^{2} \mathrm{G}_{41 / 2}^{0} \\
b^{4} \mathrm{D}^{41}-u^{4} \mathrm{~F}^{0}\end{array}$ & \\
\hline 2744. 519 & 6 & 36425.51 & -0.04 & $a^{4} \mathrm{G}_{41 / 2}-z^{2} \mathrm{~F}_{31 / 2}^{0}$ & \\
\hline 2744. 748 & 1 & 36422.46 & 0.00 & $a^{4} \mathrm{D}_{11 / 2}-w^{4} \mathrm{D}_{01 / 2}^{0}$ & \\
\hline 2745.082 & 3 & 36418.03 & $\left\{\begin{array}{r}0.00 \\
-0.05\end{array}\right.$ & $\begin{array}{l}b^{4} \mathrm{D}_{21 / 2}-u{ }^{4} \mathrm{~F}_{21 / 2}^{10} \\
b^{4} \mathrm{D}_{11}-u^{4} \mathrm{~F}_{1}^{0}\end{array}$ & \\
\hline 2745.549 & 10 & 36411.84 & -0.09 & $b^{4} \mathrm{D}_{11 / 2}-u{ }^{4} \mathbf{F}_{21 / 2}^{10 / 2}$ & \\
\hline 2745.913 & $2 h$ & 36407.01 & 0. 09 & $z^{8} \mathrm{P}_{31 / 2}^{\circ}-g^{6} \mathrm{D}_{41 / 2}$ & \\
\hline 2747.785 & $4 d$ & 36382.21 & $\left\{\begin{array}{r}0.08 \\
-0.09\end{array}\right.$ & $a{ }^{4} \mathrm{D}_{11 / 2}-z^{2} \mathrm{D}_{21 / 2}$ & \\
\hline 2749. 205 & $20 h$ & 36363.42 & $\begin{array}{r}-0.07 \\
-0.02\end{array}$ & $b^{4} \mathrm{D}_{21 / 2}^{21 / 2}-u^{4} \mathrm{~F}_{31 / 2}^{0}$ & \\
\hline 2750. 602 & 3 & 36344. 95 & 0. 19 & $a^{4} \mathrm{P}_{21 / 2}-63546_{31 / 2}^{\circ}$ & \\
\hline 2752.259 & 3 & 36323.07 & -0.04 & $a{ }^{4} \mathrm{D}_{01 / 2}-w^{4} \mathrm{D}_{0 \mathrm{w}^{\prime}}^{\circ}$ & \\
\hline
\end{tabular}


Table 1. Mn I-Classified lines-Continued

\begin{tabular}{|c|c|c|c|c|c|}
\hline 1 & 2 & 3 & 4 & 5 & 6 \\
\hline$\lambda$ & Int. & $\sigma$ & $\mathrm{o}-\mathrm{c}$ & Term designation & $\begin{array}{l}\text { Zeeman } \\
\text { type }\end{array}$ \\
\hline $\begin{array}{c}\text { A } \\
\text { Air } \\
\text { 2752. } 322 \\
2752.635 \\
2753.851 \\
2753.995 \\
2755.777\end{array}$ & $\begin{array}{l}7 \\
3 \\
3 \\
3 \mathrm{H} w \\
2\end{array}$ & $\begin{array}{c}K \\
36322.24 \\
36318.10 \\
36302.07 \\
36300.18 \\
36276.70\end{array}$ & $\begin{array}{r}K \\
-0.04 \\
-0.11 \\
0.08 \\
-0.26 \\
-0.01\end{array}$ & $\begin{array}{l}a{ }^{4} \mathrm{P}_{21 / 2}-63523_{21 / 2}^{\circ} \\
b^{4} \mathrm{D}_{21 / 2}-v^{2} \mathrm{G}_{31 / 2}^{\circ} \\
a^{4} \mathrm{P}_{01 / 2}-63583_{21 / 2,11 / 2}^{\circ} \\
b^{4} \mathrm{D}_{31 / 2}-66654_{21 / 2}^{\circ} \\
b^{4} \mathrm{D}_{31 / 2}-v^{2} \mathrm{G}_{41 / 2}^{\circ 1 / 2}\end{array}$ & \\
\hline $\begin{array}{l}2755.839 \\
2756.267\end{array}$ & $\begin{array}{l}2 \\
4\end{array}$ & $\begin{array}{l}36275.88 \\
36270.25\end{array}$ & $\begin{array}{l}0.06 \\
0.00\end{array}$ & $\begin{array}{l}a^{4} \mathrm{P}_{11 / 2}-63523_{21 / 2}^{\circ} \\
a^{4} \mathrm{D}_{11 / 2}-w^{4} \mathrm{D}_{11 / 2}^{\circ}\end{array}$ & \\
\hline 2758.950 & 4 & 36234.98 & $\left\{\begin{array}{l}-0.06 \\
-0.13\end{array}\right.$ & $\begin{array}{l}b^{4} \mathrm{D}_{21 / 2}-66654_{21 / 2} \\
a^{4} \mathrm{D}^{2}-x^{4} \mathrm{G}^{\circ}{ }^{2}\end{array}$ & \\
\hline $\begin{array}{l}2760.920 \\
2761.350\end{array}$ & $\begin{array}{c}100 h l \\
4\end{array}$ & $\begin{array}{l}36209.13 \\
36203.50\end{array}$ & $\begin{array}{l}0.00 \\
0.03\end{array}$ & $\begin{array}{l}a^{6} \mathrm{D}_{41 / 2}-t^{6} \mathrm{P}_{31 / 2}^{0} \\
a^{4} \mathrm{G}_{51 / 2}-w^{4} \mathrm{G}_{51 / 2}^{8}\end{array}$ & \\
\hline $\begin{array}{l}\text { 2761. } 797 \\
\text { 2761. } 985 \\
\text { 2762. } 853 \\
2763.665 \\
2763.87\end{array}$ & $\begin{array}{l}3 \\
2 h \\
3 h \\
3 \\
3\end{array}$ & $\begin{array}{l}36197.64 \\
36195.17 \\
36183.80 \\
36173.17 \\
36170.4\end{array}$ & $\begin{array}{l}0.04 \\
0.00 \\
0.02 \\
-0.30 \\
-0.5\end{array}$ & $\begin{array}{l}a{ }^{4} \mathrm{G}_{31 / 2}-u^{4} \mathrm{G}_{41 / 2}^{\circ} \\
a^{4} \mathrm{G}_{41 / 2}-w^{4} \mathrm{G}_{31 / 2}^{\circ} \\
a^{4} \mathrm{G}_{41 / 2}-w^{4} \mathrm{G}_{51 / 2}^{\circ} \\
a^{4} \mathrm{D}_{31 / 2}-v^{4} \mathrm{D}_{31 / 2}^{\circ} \\
a^{4} \mathrm{D}_{01 / 2}-w^{4} \mathrm{D}_{11 / 2}^{\circ}\end{array}$ & \\
\hline $\begin{array}{l}2763.907 \\
2764.027 \\
2767.450 \\
2769.410 \\
2770.242\end{array}$ & $\begin{array}{c}8 h \\
1 \\
10 \\
2 \\
3\end{array}$ & $\begin{array}{l}36170.00 \\
36168.44 \\
36123.70 \\
36098.13 \\
36087.30\end{array}$ & $\begin{array}{r}-0.02 \\
0.03 \\
0.14 \\
-0.05 \\
0.06\end{array}$ & $\begin{array}{l}a{ }^{4} \mathrm{P}_{21 / 2}-63371_{21 / 2}^{\circ} \\
b^{4} \mathrm{D}_{31 / 2}-u^{4} \mathrm{G}_{11 / 2}^{\circ} \\
a^{4} \mathrm{P}_{11 / 2}-63371_{21 / 2}^{\circ} \\
a^{4} \mathrm{D}_{11 / 2}-x^{4} \mathrm{G}_{21 / 2}^{\circ} \\
a^{4} \mathrm{P}_{21 / 2}-x^{2} \mathrm{~F}_{31 / 2}^{2}\end{array}$ & \\
\hline $\begin{array}{l}\text { 2771. } 430 \\
2772.032 \\
2773.021 \\
2773.659 \\
2774.289\end{array}$ & $\begin{array}{r}30 \\
2 \\
5 \\
10 \\
1\end{array}$ & $\begin{array}{l}36071.83 \\
36063.99 \\
36051.13 \\
36042.84 \\
36034.66\end{array}$ & $\begin{array}{r}0.03 \\
-0.06 \\
-0.02 \\
-0.04 \\
0.00\end{array}$ & $\begin{array}{l}a{ }^{6} \mathrm{D}_{41 / 2}-y{ }^{4} \mathrm{D}_{31 / 2}^{\circ} \\
a^{4} \mathrm{D}_{31 / 2}-x^{4} \mathrm{~F}_{21 / 2}^{\circ} \\
a^{4} \mathrm{D}_{21 / 2}-w^{4} \mathrm{D}_{21 / 2}^{\circ} \\
a^{4} \mathrm{D}_{31 / 2}-w^{4} \mathrm{D}_{31 / 2}^{\circ} \\
b^{4} \mathrm{D}_{2112}-u^{4} \mathrm{G}_{31 / 2}^{3}\end{array}$ & \\
\hline $\begin{array}{l}\text { 2776. } 218 \\
2777.464 \\
2778.544 \\
2779.993 \\
2781.733\end{array}$ & $\begin{array}{l}80 h l \\
6 \\
60 h \\
40 \\
1\end{array}$ & $\begin{array}{l}36009.62 \\
35993.47 \\
35979.47 \\
35960.72 \\
35938.23\end{array}$ & $\begin{array}{r}0.04 \\
-0.09 \\
0.05 \\
-0.05 \\
0.07\end{array}$ & $\begin{array}{l}a^{6} \mathrm{D}_{31 / 2}-t^{6} \mathrm{P}_{21 / 2}^{\circ} \\
a^{4} \mathrm{D}_{31 / 2}-x{ }^{4} \mathrm{~F}_{31 / 2}^{\circ} \\
a^{6} \mathrm{D}_{31 / 2}-t^{6} \mathrm{P}_{31 / 2}^{0} \\
a^{4} \mathrm{D}_{31 / 2}-x^{4} \mathrm{~F}_{41 / 2}^{0} \\
a^{4} \mathrm{P}_{21 / 2}-x^{2} \mathrm{~F}_{21 / 2}^{0}\end{array}$ & \\
\hline $\begin{array}{l}2782.259 \\
2782.711 \\
2783.080 \\
2785.334 \\
2786.185\end{array}$ & $\begin{array}{l}1 \\
50 \mathrm{H} \\
10 \\
1 \\
3\end{array}$ & $\begin{array}{l}35931.44 \\
35925.60 \\
35920.84 \\
35891.77 \\
35880.81\end{array}$ & $\begin{array}{r}-0.16 \\
0.00 \\
-0.10 \\
0.07 \\
-0.02\end{array}$ & $\begin{array}{c}a{ }^{4} \mathrm{D}_{21 / 2}-v^{4} \mathrm{D}_{21 / 2}^{\circ} \\
a^{6} \mathrm{D}_{41 / 2}-w^{6} \mathrm{~F}_{51 / 2}^{51 / 2} \\
a^{4} \mathrm{D}_{21 / 2}-v^{4} \mathrm{D}_{31 / 2}^{\circ} \\
a^{4} \mathrm{P}_{11 / 2}-x^{2} \mathrm{~F}_{21 / 2}^{0} \\
a^{4} \mathrm{D}_{11 / 2}-w^{4} \mathrm{D}_{21 / 2}^{0}\end{array}$ & \\
\hline $\begin{array}{l}\text { 2786. } 266 \\
2787.264 \\
2787.813 \\
2788.682 \\
2789.192\end{array}$ & $\begin{array}{c}3 \\
2 \\
15 h \\
3 \\
25\end{array}$ & $\begin{array}{l}35879.77 \\
35866.93 \\
35859.86 \\
35848.68 \\
35842.13\end{array}$ & $\begin{array}{r}0.03 \\
-0.02 \\
0.01 \\
-0.09 \\
0.04\end{array}$ & $\begin{array}{l}a^{4} \mathrm{P}_{21 / 2}-y{ }^{2} \mathrm{D}_{21 / 2}^{0} \\
a^{4} \mathrm{D}_{21 / 2}-x{ }^{4} \mathrm{~F}_{11 / 2}^{0} \\
a^{6} \mathrm{D}_{21 / 2}-t^{6} \mathrm{P}_{11 / 2} \\
a^{4} \mathrm{D}_{11 / 2}-u{ }^{4} \mathrm{P}_{01 / 2}^{\circ} \\
a^{6} \mathrm{D}_{31 / 2}-y \\
{ }^{4} \mathrm{D}_{31 / 2}\end{array}$ & 6 \\
\hline $\begin{array}{l}2789.355 \\
2789.731 \\
2790.353 \\
2790.925 \\
2791.085\end{array}$ & $\begin{array}{l}15 h w \\
3 \\
30 \\
4 \\
20\end{array}$ & $\begin{array}{l}35840.03 \\
35835.20 \\
35827.21 \\
35819.87 \\
35817.82\end{array}$ & $\begin{array}{r}-0.03 \\
-0.05 \\
0.00 \\
-0.06 \\
0.01\end{array}$ & $\begin{array}{l}a{ }^{6} \mathrm{D}_{21 / 2}-t^{6} \mathrm{P}_{21 / 2}^{\circ} \\
a^{4} \mathrm{D}_{21 / 2}-u^{4} \mathrm{P}_{11 / 2}^{\circ} \\
a^{6} \mathrm{D}_{31 / 2}-y{ }^{4} \mathrm{D}_{21 / 2}^{\circ} \\
a^{4} \mathrm{D}_{31 / 2}-u{ }^{4} \mathrm{P}_{21 / 2}^{0} \\
a^{6} \mathrm{D}_{41 / 2}-x{ }^{6} \mathrm{D}_{31 / 2}^{0}\end{array}$ & \\
\hline $\begin{array}{l}\text { 2791. } 584 \\
\text { 2791. } 707 \\
2793.24 \\
2794.817 \\
2796.938\end{array}$ & $\begin{array}{r}4 \\
2 h \\
6 h \\
10000 \mathrm{R} \\
5\end{array}$ & $\begin{array}{l}35811.42 \\
35809.84 \\
35790.2 \\
35769.99 \\
35742.88\end{array}$ & $\begin{array}{r}-0.10 \\
-0.06 \\
-0.2 \\
0.02 \\
-0.01\end{array}$ & 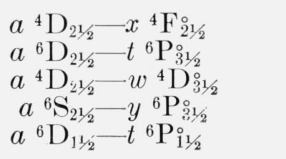 & 4 \\
\hline $\begin{array}{l}2797.194 \\
2798.270 \\
2799.841 \\
2800.63 \\
2801.084\end{array}$ & $\begin{array}{c}3 \\
8000 \mathrm{R} \\
50 \\
6 \mathrm{H} \\
6000 \mathrm{R}\end{array}$ & $\begin{array}{l}35740.88 \\
35725.86 \\
35705.81 \\
35695.75 \\
35689.97\end{array}$ & $\begin{array}{r}-0.15 \\
0.01 \\
-0.01 \\
0.00 \\
-0.01\end{array}$ & $\begin{array}{c}a^{4} \mathrm{D}_{21 / 2}-x{ }^{4} \mathrm{~F}_{31 / 2}^{\circ} \\
a{ }^{6} \mathrm{~S}_{21 / 2}-y \\
{ }^{6} \mathrm{P}_{21 / 2}^{0} \\
a^{6} \mathrm{D}_{41 / 2}-x \\
a^{6} \mathrm{D}_{41 / 2}^{\circ} \\
{ }^{6} \mathrm{D}_{31 / 2}-w^{6} \mathrm{~F}_{41 / 2}^{0} \\
a^{6} \mathrm{~S}_{21 / 2}-y{ }^{6} \mathrm{P}_{11 / 2}^{\circ}\end{array}$ & $6, \stackrel{6}{7 b}$ \\
\hline
\end{tabular}


Table 1. Mn I-Classified lines-Continued

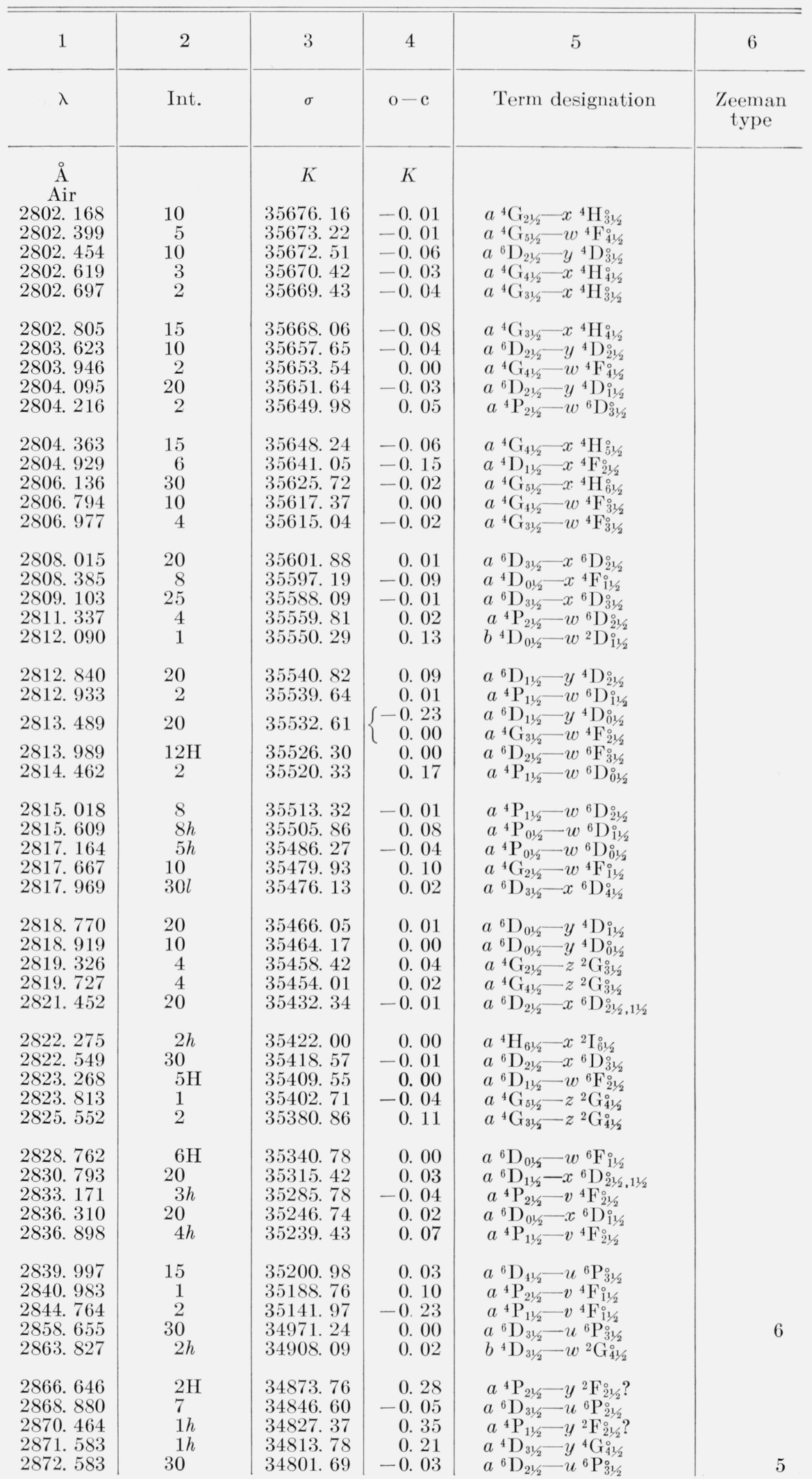


Table 1. Mn I-Classified lines-Continued

\begin{tabular}{|c|c|c|c|c|c|}
\hline 1 & 2 & 3 & 4 & 5 & 6 \\
\hline$\lambda$ & Int. & $\sigma$ & $\mathrm{o}-\mathrm{c}$ & Term designation & $\begin{array}{c}\text { Zeeman } \\
\text { type }\end{array}$ \\
\hline $\begin{array}{c}\stackrel{\AA}{\text { Air }} \\
\text { 2880. } 270 \\
2882.899 \\
2890.388 \\
2891.945 \\
\text { 2892. } 388\end{array}$ & $\begin{array}{l}1 h \\
20 \\
1 h \\
2 h \\
2\end{array}$ & $\begin{array}{c}K \\
34708.81 \\
34677.16 \\
34587.31 \\
34568.69 \\
34563.39\end{array}$ & $\begin{array}{r}K \\
0.08 \\
0.03 \\
-0.18 \\
0.08 \\
-0.09\end{array}$ & $\begin{array}{l}a^{4} \mathrm{G}_{21 / 2}-w^{4} \mathrm{D}_{11 / 2}^{\circ} \\
a^{6} \mathrm{D}_{21 / 2}-u^{6} \mathrm{P}_{21 / 2}^{\circ} \\
a^{4} \mathrm{D}_{21 / 2}-y^{4} \mathrm{G}_{31 / 2}^{\circ} \\
b^{4} \mathrm{D}_{21 / 2}-w^{2} \mathrm{~F}_{31 / 2}^{\circ} \\
a^{6} \mathrm{D}_{21 / 2}-u^{6} \mathrm{P}_{11 / 2}^{\circ}\end{array}$ & \\
\hline $\begin{array}{l}2892.493 \\
2892.657 \\
2894.625 \\
2895.188 \\
2897.428\end{array}$ & $\begin{array}{r}5 \\
20 \\
10 \\
8 \\
5\end{array}$ & $\begin{array}{l}34562.13 \\
34560.19 \\
34536.69 \\
34529.97 \\
34503.28\end{array}$ & $\begin{array}{r}-0.01 \\
0.02 \\
0.03 \\
0.01 \\
0.01\end{array}$ & $\begin{array}{l}a^{4} \mathrm{G}_{51 / 2}-z^{2} \mathrm{I}_{51 / 2}^{\circ} \\
a^{6} \mathrm{D}_{11 / 2}-u^{6} \mathrm{P}_{21 / 2}^{\circ} \\
a^{4} \mathrm{G}_{21 / 2}-x{ }^{4} \mathrm{G}_{21 / 2}^{\circ} \\
a^{4} \mathrm{G}_{31 / 2}-x^{4} \mathrm{G}_{21 / 2}^{\circ}{ }^{1} \\
a^{4} \mathrm{G}_{21 / 2}-x{ }^{4} \mathrm{G}_{31 / 2}^{\circ}\end{array}$ & 5 \\
\hline $\begin{array}{l}2897.651 \\
2897.797 \\
2897.990 \\
2900.545\end{array}$ & $\begin{array}{r}1 \\
15 \\
10 \\
20\end{array}$ & $\begin{array}{l}34500.63 \\
34498.89 \\
34496.59 \\
34466.20\end{array}$ & $\begin{array}{r}-0.09 \\
0.01 \\
0.02 \\
0.00\end{array}$ & $\begin{array}{l}b^{4} \mathrm{D}_{31 / 2}-v{ }^{4} \mathrm{H}_{31 / 2}^{0} \\
a^{4} \mathrm{G}_{41 / 2}-x{ }^{4} \mathrm{G}_{31 / 2} \\
a^{4} \mathrm{G}_{31 / 2}-x \\
a^{4} \mathrm{G}_{31 / 2}^{\circ} \\
a^{4} \mathrm{G}_{51 / 2}-x{ }^{4} \mathrm{G}_{41 / 2}^{0}\end{array}$ & 5 \\
\hline 2902. 203 & 25 & 34446.51 & $\left\{\begin{array}{r}-0.01 \\
0.00\end{array}\right.$ & $\begin{array}{l}a^{6} \mathrm{D}_{11 / 2}-u{ }^{6} \mathrm{P}_{11 / 2}^{1} \\
a^{4} \mathrm{G}_{41 / 2}-x{ }^{4} \mathrm{G}_{41 / 2}^{0}\end{array}$ & \\
\hline $\begin{array}{l}\text { 2902. } 399 \\
2905.825 \\
2906.340 \\
2907.214 \\
2907.993\end{array}$ & $\begin{array}{l}5 \\
1 h \\
4 h \\
40 \\
15\end{array}$ & $\begin{array}{l}34444.19 \\
34403.58 \\
34397.49 \\
34387.15 \\
34377.93\end{array}$ & $\begin{array}{r}-0.01 \\
-0.02 \\
-0.01 \\
-0.01 \\
0.08\end{array}$ & $\begin{array}{l}a^{4} \mathrm{G}_{31 / 2}-x{ }^{4} \mathrm{G}_{41 / 2}^{0} \\
b{ }^{4} \mathrm{D}_{21 / 2}-w^{2} \mathrm{~F}_{21 / 2}^{0} \\
b^{4} \mathrm{D}_{11 / 2}-w^{3} \mathrm{~F}_{21 / 2}^{2} \\
a^{4} \mathrm{G}_{51 / 2}-x{ }^{4} \mathrm{G}_{51 / 2}^{\circ} \\
a^{6} \mathrm{D}_{01 / 2}-u^{6} \mathrm{P}_{11 / 2}^{0}\end{array}$ & 6 \\
\hline $\begin{array}{l}\text { 2908. } 878 \\
2909.630 \\
2910.242 \\
2911.839 \\
2912.000\end{array}$ & $\begin{array}{c}10 \\
1 \\
3 \\
1 h \\
2 h\end{array}$ & $\begin{array}{l}34367.47 \\
34358.59 \\
34351.37 \\
34332.53 \\
34330.63\end{array}$ & $\begin{array}{r}0.00 \\
-0.14 \\
-0.01 \\
-0.02 \\
-0.13\end{array}$ & $\begin{array}{l}a^{4} \mathrm{G}_{41 / 2}-x^{4} \mathrm{G}_{51 / 2}^{0} \\
b^{4} \mathrm{D}_{31 / 2}-u^{4} \mathrm{D}_{21 / 2}^{\circ} \\
a^{4} \mathrm{G}_{51 / 2}-z^{2} \mathrm{I}_{61 / 2}^{\circ} \\
z^{8} \mathrm{P}_{21 / 2}^{\circ}-f{ }^{6} \mathrm{D}_{11 / 2}^{\circ} \\
z^{8} \mathrm{P}_{21 / 2}^{0}-f^{6} \mathrm{D}_{21 / 2}^{0}\end{array}$ & \\
\hline $\begin{array}{l}\text { 2912. } 226 \\
2913.518 \\
2914.599 \\
2916.375 \\
2917.637\end{array}$ & $\begin{array}{c}3 h \\
1 \\
600 h w \\
1 h \\
1\end{array}$ & $\begin{array}{l}34327.97 \\
34312.74 \\
34300.02 \\
34279.13 \\
34264.31\end{array}$ & $\begin{array}{r}0.02 \\
0.13 \\
0.00 \\
0.07 \\
-0.03\end{array}$ & $\begin{array}{l}z^{8} \mathrm{P}_{21 / 2}^{\circ}-f^{6} \mathrm{D}_{31 / 2} \\
a^{4} \mathrm{G}_{31 / 2}-w^{4} \mathrm{D}_{21 / 2}^{2} \\
z^{8} \mathrm{P}_{21 / 2}^{\circ}-f^{8} \mathrm{D}^{8} \\
a^{4} \mathrm{P}_{21 / 2}-w^{4} \mathrm{G}_{31 / 2}^{\circ} \\
b^{4} \mathrm{D}_{21 / 2}-u^{4} \mathrm{D}_{11 / 2}^{0}\end{array}$ & \\
\hline $\begin{array}{l}\text { 2919. } 122 \\
2920.458 \\
2920.599 \\
2921.112 \\
2923.145\end{array}$ & $\begin{array}{l}8 \\
8 \\
5 \\
1 \\
2\end{array}$ & $\begin{array}{l}34246.88 \\
34231.21 \\
34229.56 \\
34223.55 \\
34199.75\end{array}$ & $\begin{array}{r}0.03 \\
-0.02 \\
-0.03 \\
0.32 \\
-0.01\end{array}$ & $\begin{array}{l}a^{4} \mathrm{G}_{21 / 2}-v{ }^{4} \mathrm{D}_{11 / 2}^{\circ} \\
b^{4} \mathrm{D}_{31 / 2}-x^{2} \mathrm{G}_{41 / 2}^{\circ} \\
b^{4} \mathrm{D}_{21 / 2}-x^{2} \mathrm{G}_{31 / 2}^{\circ} \\
a^{4} \mathrm{P}_{11 / 2}-w^{4} \mathrm{G}_{21 / 2}^{\circ} \\
a^{4} \mathrm{G}_{-1 / 2}-v^{4} \mathrm{D}_{21 / 2}^{0}\end{array}$ & 4 \\
\hline $\begin{array}{l}\text { 2923. } 229 \\
2923.577 \\
\text { 2923. } 715 \\
2924.430 \\
2924.629\end{array}$ & $\begin{array}{c}2 h \\
3 h \\
10 \\
10 \\
2\end{array}$ & $\begin{array}{l}34198.76 \\
34194.69 \\
34193.08 \\
34184.72 \\
34182.39\end{array}$ & $\begin{array}{r}-0.01 \\
-0.06 \\
0.02 \\
0.01 \\
-0.01\end{array}$ & $\begin{array}{l}z^{8} \mathrm{P}_{31 / 2}^{\circ}-f{ }^{6} \mathrm{D}_{31 / 2} \\
z^{8} \mathrm{P}_{31 / 2}^{\circ}-f{ }^{6} \mathrm{D}_{41 / 2} \\
a^{4} \mathrm{G}_{31 / 2}-v \\
{ }^{4} \mathrm{D}_{21 / 2}^{0} \\
a^{4} \mathrm{G}_{41 / 2}-v^{4} \mathrm{D}_{31 / 2}^{0} \\
a^{4} \mathrm{G}_{31 / 2}-v^{4} \mathrm{D}_{31 / 2}^{8}\end{array}$ & \\
\hline $\begin{array}{l}\text { 2925. } 58 \\
2928.678 \\
2930.245 \\
2933.442 \\
2934.020\end{array}$ & $\begin{array}{l}500 h w \\
40 \\
20 \\
3 \\
30\end{array}$ & $\begin{array}{l}34171.3 \\
34135.14 \\
34116.89 \\
34079.71 \\
34072.99\end{array}$ & $\begin{array}{c}-0.2 \\
0.03 \\
0.00 \\
0.03 \\
0.01\end{array}$ & $\begin{array}{l}z^{8} \mathrm{P}_{31 / 2}^{\circ}-f^{8} \mathrm{D}_{41 / 2} \\
a^{4} \mathrm{G}_{21 / 2}-x^{4} \mathrm{~F}_{11 / 2}^{0} \\
a^{6} \mathrm{D}_{41 / 2}-x \\
a^{6} \mathrm{~F}_{51 / 2}^{\circ} \\
a^{4} \mathrm{G}_{21 / 2}-x \\
a^{4} \mathrm{~F}_{21 / 2}^{\circ} \mathrm{G}_{31 / 2}-x \\
{ }^{4} \mathrm{~F}_{21 / 2}^{\circ}\end{array}$ & $\mathrm{P}-\mathrm{B}$ \\
\hline $\begin{array}{l}\text { 2935. } 643 \\
2935.844 \\
2936.156 \\
2938.496 \\
2939.904\end{array}$ & $\begin{array}{l}15 \\
3 \\
10 \\
2 h \\
20\end{array}$ & $\begin{array}{l}34054.15 \\
34051.82 \\
34048.20 \\
34021.09 \\
34004.80\end{array}$ & $\begin{array}{l}0.03 \\
0.01 \\
0.00 \\
0.07 \\
0.00\end{array}$ & $\begin{array}{c}a^{4} \mathrm{G}_{41 / 2}-w^{4} \mathrm{D}_{31 / 2}^{\circ} \\
a^{4} \mathrm{G}_{31 / 2}-w^{4} \mathrm{D}_{31 / 2}^{0} \\
a^{6} \mathrm{D}_{41 / 2}-x^{6} \mathrm{~F}_{41 / 2}^{\circ} \\
z^{8} \mathrm{P}_{41 / 2}^{\circ}-f^{6} \mathrm{D}_{41 / 2}^{\circ} \\
a^{4} \mathrm{G}_{41 / 2}-x{ }^{4} \mathrm{~F}_{31 / 2}^{0}\end{array}$ & 6 \\
\hline $\begin{array}{l}\text { 2940. } 331 \\
\text { 2940. } 483 \\
\text { 2941. } 038 \\
\text { 2941. } 681 \\
\text { 2942. } 740\end{array}$ & $\begin{array}{c}400 h w \\
2 \\
40 \\
5 \\
8\end{array}$ & $\begin{array}{l}33999.86 \\
33997.91 \\
33991.69 \\
33984.26 \\
33972.03\end{array}$ & $\begin{array}{l}0.00 \\
0.2 \\
-0.01 \\
-0.04 \\
0.02\end{array}$ & $\begin{array}{c}z^{8} \mathrm{P}_{41 / 2}^{\circ}-f^{8} \mathrm{D}_{51 / 2} \\
z^{8} \mathrm{P}_{41 / 2}^{\circ}-f^{8} \mathrm{D}_{41 / 2} \\
a^{4} \mathrm{G}_{51 / 2}-x \\
a^{4} \mathrm{~F}_{41 / 2}^{\circ} \\
a^{6} \mathrm{D}_{11 / 2}-x{ }^{4} \mathrm{P}_{01 / 2}^{\circ} \\
a^{4} \mathrm{G}_{41 / 2}-x{ }^{4} \mathrm{~F}_{41 / 2}^{\circ}\end{array}$ & 4 \\
\hline
\end{tabular}


Table 1. Mn I-Classified lines-Continued

\begin{tabular}{|c|c|c|c|c|c|}
\hline 1 & 2 & 3 & 4 & 5 & 6 \\
\hline$\lambda$ & Int. & $\sigma$ & $o-c$ & Term designation & $\begin{array}{l}\text { Zeeman } \\
\text { type }\end{array}$ \\
\hline $\begin{array}{c}\AA \\
\text { Air } \\
2943.550 \\
2947.634 \\
2950.979 \\
2953.008 \\
2956.101\end{array}$ & $\begin{array}{r}1 \\
3 \\
3 \\
10 \\
20\end{array}$ & $\begin{array}{c}K \\
33962.68 \\
33915.63 \\
33877.19 \\
33853.91 \\
33818.49\end{array}$ & $\begin{array}{l}K \\
0.02 \\
0.00 \\
0.02 \\
0.02 \\
0.00\end{array}$ & $\begin{array}{l}a{ }^{6} \mathrm{D}_{41 / 2}-x^{6} \mathrm{~F}_{31 / 2}^{0} \\
a^{6} \mathrm{D}_{01 / 2}-x^{4} \mathrm{P}_{01 / 2}^{\circ} \\
a^{6} \mathrm{D}_{11 / 2}-x^{4} \mathrm{P}_{11 / 2}^{\circ} \\
a^{6} \mathrm{D}_{21 / 2}-x{ }^{4} \mathrm{P}_{21 / 2}^{0} \\
a^{6} \mathrm{D}_{31 / 2}-x^{6} \mathrm{~F}_{41 / 2}^{0}\end{array}$ & 4 \\
\hline $\begin{array}{l}2956.971 \\
2963.250 \\
2963.606 \\
2966.374 \\
2970.956\end{array}$ & $\begin{array}{r}10 \\
10 \\
20 \\
1 \\
4\end{array}$ & $\begin{array}{l}33808.54 \\
33736.90 \\
33732.85 \\
33701.38 \\
33649.40\end{array}$ & $\begin{array}{r}0.04 \\
-0.03 \\
-0.10 \\
0.12 \\
-0.02\end{array}$ & $\begin{array}{c}a{ }^{6} \mathrm{D}_{01 / 2}-x^{4} \mathrm{P}_{11 / 2} \\
a{ }^{6} \mathrm{D}_{11 / 2}-x^{4} \mathrm{P}_{21 / 2}^{0} \\
a^{6} \mathrm{D}_{31 / 2}-x^{6} \mathrm{~F}_{31 / 2}^{0} \\
a{ }^{4} \mathrm{P}_{21 / 2}-w^{4} \mathrm{~F}_{31 / 2}^{0} \\
a^{6} \mathrm{D}_{31 / 2}-x^{6} \mathrm{~F}_{21 / 2}^{0}\end{array}$ & 6 \\
\hline $\begin{array}{l}2974.089 \\
2976.588 \\
2976.987 \\
2977.190 \\
2977.303\end{array}$ & $\begin{array}{r}20 \\
1 \\
1 \\
1 \\
3\end{array}$ & $\begin{array}{l}33613.96 \\
33585.74 \\
33581.23 \\
33578.95 \\
33577.67\end{array}$ & $\begin{array}{r}0.00 \\
-0.01 \\
0.00 \\
0.03 \\
0.02\end{array}$ & $\begin{array}{l}a{ }^{4} \mathrm{H}_{61 / 2}-t^{4} \mathrm{G}_{51 / 2}^{\circ} \\
a^{4} \mathrm{G}_{51 / 2}-z^{4} \mathrm{I}_{51 / 2}^{\circ} \\
a^{4} \mathrm{G}_{41 / 2}-z^{4} \mathrm{I}_{41 / 2}^{\circ} \\
a^{4} \mathrm{G}_{31 / 2}-z^{4} \mathrm{I}_{41 / 2}^{\circ} \\
a^{4} \mathrm{G}_{51 / 2}-z^{4} \mathrm{I}_{61 / 2}^{\circ}\end{array}$ & 4 \\
\hline $\begin{array}{l}2977.755 \\
2977.922 \\
2978.114 \\
2978.333 \\
2978.566\end{array}$ & $\begin{array}{l}1 \\
1 \\
8 h \\
2 \\
15\end{array}$ & $\begin{array}{l}33572.57 \\
33570.69 \\
33568.53 \\
33566.06 \\
33563.44\end{array}$ & $\begin{array}{l}0.22 \\
0.3 \\
-0.12 \\
0.00 \\
0.01\end{array}$ & $\begin{array}{l}a^{4} \mathrm{P}_{11 / 2}-w^{4} \mathrm{~F}_{21 / 2}^{0} \\
a^{4} \mathrm{D}_{31 / 2}-u^{6} \mathrm{~F}^{0} \\
a^{4} \mathrm{H}_{51 / 2}-t^{4} \mathrm{G}_{41 / 2}^{0} \\
a^{4} \mathrm{G}_{41 / 2}-z^{4} \mathrm{I}_{51 / 2}^{\circ} \\
a^{6} \mathrm{D}_{21 / 2}-x^{6} \mathrm{~F}_{31 / 2}^{0}\end{array}$ & \\
\hline $\begin{array}{l}2979.994 \\
2980.493 \\
2984.002 \\
2985.992 \\
2986.407\end{array}$ & $\begin{array}{c}8 h \\
2 h \\
2 \\
20 \\
2 h\end{array}$ & $\begin{array}{l}33547.35 \\
33541.74 \\
33502.29 \\
33479.97 \\
33475.32\end{array}$ & $\begin{array}{r}-0.11 \\
0.14 \\
-0.03 \\
0.07 \\
0.05\end{array}$ & $\begin{array}{l}a{ }^{4} \mathrm{H}_{41 / 2}-t^{4} \mathrm{G}_{31 / 2}^{0} \\
a{ }^{4} \mathrm{H}_{31 / 2}-t^{4} \mathrm{G}_{21 / 2}^{0} \\
a^{4} \mathrm{H}_{51 / 2}-t^{4} \mathrm{G}_{51 / 2}^{0} \\
a^{6} \mathrm{D}_{21 / 2}-x^{6} \mathrm{~F}_{21 / 2}^{0} \\
a^{4} \mathrm{H}_{41 / 2}-t^{4} \mathrm{G}_{41 / 2}^{0}\end{array}$ & 6 \\
\hline $\begin{array}{l}\text { 2987. } 052 \\
2990.135 \\
2991.387 \\
2992.109 \\
2996.183\end{array}$ & $\begin{array}{l}1 h \\
1 \\
1 \\
5 \\
2\end{array}$ & $\begin{array}{l}33468.10 \\
33433.58 \\
33419.59 \\
33411.53 \\
33366.10\end{array}$ & $\begin{array}{r}0.01 \\
0.00 \\
-0.02 \\
0.00 \\
0.08\end{array}$ & $\begin{array}{l}a{ }^{4} \mathrm{H}_{31 / 2}-t^{4} \mathrm{G}_{31 / 2}^{\circ} \\
b{ }^{4} \mathrm{D}_{01 / 2}-x^{2} \mathrm{D}_{11 / 2}^{112} \\
b{ }^{4} \mathrm{D}_{11 / 2}-x^{2} \mathrm{D}_{11 / 2}^{\circ} \\
a^{6} \mathrm{D}_{21 / 2}-x^{6} \mathrm{~F}_{11 / 2}^{\circ} \\
a^{4} \mathrm{H}_{61 / 2}-w^{2} \mathrm{H}_{51 / 2}^{\circ}\end{array}$ & \\
\hline $\begin{array}{l}\text { 2996. } 470 \\
2997.826 \\
2998.056 \\
2998.600 \\
3002.378\end{array}$ & $\begin{array}{l}10 \\
6 \mathrm{H} \\
1 \\
1 h \\
2 h w\end{array}$ & $\begin{array}{l}\text { 33362. } 90 \\
33347.81 \\
33345.25 \\
33339.20 \\
33297.25\end{array}$ & $\begin{array}{r}-0.04 \\
0.07 \\
-0.04 \\
0.01 \\
0.03\end{array}$ & $\begin{array}{l}a{ }^{6} \mathrm{D}_{11 / 2}-x^{6} \mathrm{~F}_{21 / 2}^{\circ}{ }^{\circ} \\
a{ }^{4} \mathrm{~F}_{41 / 2}-u^{2} \mathrm{G}_{41 / 2}^{1} \\
b^{4} \mathrm{D}_{21 / 2}-x^{2} \mathrm{D}_{21 / 2}^{\circ} \\
b^{4} \mathrm{D}_{11 / 2}-x^{2} \mathrm{D}_{21 / 2}^{2} \\
a^{4} \mathrm{~F}_{31 / 2}-u^{2} \mathrm{G}_{31 / 2}^{0}\end{array}$ & $6, \stackrel{4}{7 b}$ \\
\hline $\begin{array}{l}3002.616 \\
3003.125 \\
3006.629 \\
3007.102 \\
3007.650\end{array}$ & $\begin{array}{l}20 \\
2 \\
4 \\
7 \mathrm{H} \\
80\end{array}$ & $\begin{array}{l}\text { 33294. } 61 \\
33288.97 \\
33250.18 \\
33244.95 \\
33238.89\end{array}$ & $\begin{array}{r}0.04 \\
-0.04 \\
0.02 \\
-0.12 \\
0.03\end{array}$ & $\begin{array}{l}a{ }^{6} \mathrm{D}_{11 / 2}-x{ }^{6} \mathrm{~F}_{11 / 2}^{0} \\
a^{6} \mathrm{D}_{41 / 2}-y{ }^{4} \mathrm{~F}_{41 / 2}^{\circ} \\
a^{6} \mathrm{D}_{11 / 2}-x{ }^{6} \mathrm{~F}_{01 / 2}^{\circ} \\
a^{4} \mathrm{~F}_{31 / 2}-u^{2} \mathrm{G}_{41 / 2}^{\circ} \\
a^{4} \mathrm{G}_{21 / 2}-y \\
{ }^{4} \mathrm{H}_{31 / 2}^{0}\end{array}$ & 6 \\
\hline $\begin{array}{l}\text { 3008. } 050 \\
3008.258 \\
3008.822 \\
3009.378 \\
3011.165\end{array}$ & $\begin{array}{r}3 \\
40 \\
4 \\
5 \\
50\end{array}$ & $\begin{array}{l}33234.47 \\
33232.17 \\
33225.95 \\
33219.80 \\
33200.09\end{array}$ & $\begin{array}{l}0.00 \\
0.01 \\
0.05 \\
0.02 \\
0.00\end{array}$ & $\begin{array}{l}a^{4} \mathrm{G}_{41 / 2}-y{ }^{4} \mathrm{H}_{31 / 2}^{0} \\
a^{4} \mathrm{G}_{31 / 2}-y \\
{ }^{4} \mathrm{H}_{31 / 2}^{\circ} \\
a^{6} \mathrm{D}_{01 / 2}-x \\
a^{6} \mathrm{~F}_{11 / 2}^{0} \\
a^{4} \mathrm{G}_{51 / 2}-y \\
a^{4} \mathrm{H}_{41 / 2}^{\circ} \mathrm{G}_{41 / 2}-y \\
{ }^{4} \mathrm{H}_{41 / 2}^{0}\end{array}$ & 5 \\
\hline $\begin{array}{l}3011.376 \\
3012.854 \\
3013.933 \\
3014.666 \\
3015.183\end{array}$ & $\begin{array}{c}70 \\
8 \\
1 h \\
70 \\
1\end{array}$ & $\begin{array}{l}33197.76 \\
33181.48 \\
33169.60 \\
33161.54 \\
33155.85\end{array}$ & $\begin{array}{r}-0.02 \\
-0.01 \\
-0.01 \\
-0.02 \\
0.04\end{array}$ & $\begin{array}{c}a{ }^{4} \mathrm{G}_{31 / 2}-y{ }^{4} \mathrm{H}_{41 / 2}^{\circ} \\
a^{6} \mathrm{D}_{01 / 2}-x{ }^{6} \mathrm{~F}_{01 / 2}^{01 / 2} \\
b^{4} \mathrm{D}_{31 / 2}-63523_{21 / 2}^{\circ}-63 \\
a^{4} \mathrm{G}_{51 / 2}-y{ }^{4} \mathrm{H}_{51 / 2}^{\circ} \\
b^{4} \mathrm{P}_{21 / 2}-66981_{31 / 2}^{\circ}\end{array}$ & 6 \\
\hline 3015. 915 & 2 & 33147.80 & $\left\{\begin{array}{l}0.16 \\
0.2\end{array}\right.$ & $\begin{array}{l}a{ }^{4} \mathrm{P}_{11 / 2}-z^{2} \mathrm{D}_{11 / 2}^{\circ} \\
a^{4} \mathrm{D}_{11 / 2}-u^{6} \mathrm{~F}^{0}\end{array}$ & \\
\hline $\begin{array}{l}3016.454 \\
3019.018 \\
3021.673 \\
3022.743\end{array}$ & $\begin{array}{c}100 \\
2 \\
2 h \\
120\end{array}$ & $\begin{array}{l}33141.88 \\
33113.74 \\
33084.64 \\
33072.93\end{array}$ & $\begin{array}{r}0.01 \\
-0.05 \\
0.11 \\
0.00\end{array}$ & $\begin{array}{l}a{ }^{4} \mathrm{G}_{41 / 2}-y{ }^{4} \mathrm{H}_{51 / 2}^{\circ} \\
a{ }^{4} \mathrm{P}_{01 / 2}-z{ }^{2} \mathrm{D}_{11 / 2}^{\circ} \\
b{ }^{4} \mathrm{P}_{21 / 2}-66910_{21 / 2}^{\circ} \\
a^{4} \mathrm{G}_{51 / 2}-y{ }^{4} \mathrm{H}_{61 / 2}^{\circ}\end{array}$ & 4 \\
\hline
\end{tabular}


Table 1. Mn I-Classified lines-Continued

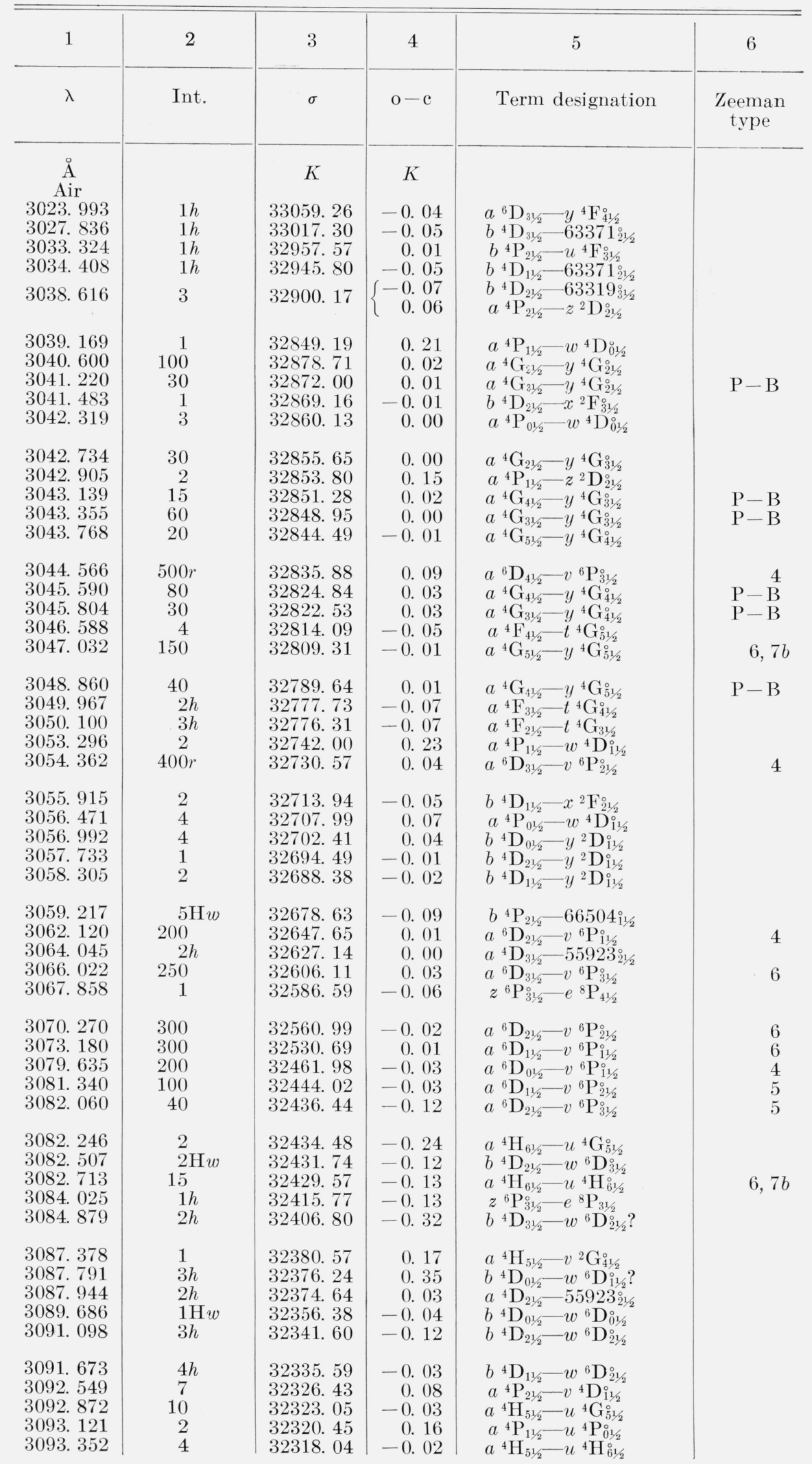


Table 1. Mn I-Classified lines - Continued

\begin{tabular}{|c|c|c|c|c|c|}
\hline 1 & 2 & 3 & 4 & 5 & 6 \\
\hline$\lambda$ & Int. & $\sigma$ & $\mathrm{o}-\mathrm{c}$ & Term designation & $\begin{array}{l}\text { Zeeman } \\
\text { type }\end{array}$ \\
\hline $\begin{array}{c}\text { A } \\
\text { Air }\end{array}$ & & $K$ & $K$ & & \\
\hline 3093. 467 & 1 & 32316.84 & 0. 24 & $b{ }^{4} \mathbf{D}_{31 / 2}-w^{6} \mathbf{D}_{41 / 2}^{\circ}$ & \\
\hline 3093. 689 & 2 & 32314.52 & -0.03 & $a^{4} \mathrm{H}_{31 / 2}^{0}-v^{2} \mathrm{G}_{31 / 2}^{0}$ & \\
\hline 3094. 818 & 1 & 32302. 73 & 0. 01 & $b^{4} \mathrm{G}_{51 / 2}-v^{2} \mathrm{H}_{51 / 2}^{0}$ & \\
\hline $\begin{array}{l}\text { 3095. } 244 \\
3096.384\end{array}$ & 1 & $\begin{array}{l}32298.29 \\
32286.39\end{array}$ & $\begin{array}{r}0.01 \\
-0.05\end{array}$ & $z^{6} \mathrm{P}_{21 / 2}^{0}-e^{8} \mathrm{P}_{21 / 2}$ & \\
\hline 3096.384 & & 32286.39 & -0.05 & $a^{4} \mathrm{P}_{01 / 2}-u^{4} \mathrm{P}_{01 / 2}^{0}$ & \\
\hline 3097. 010 & 20 & 32279.87 & $\left\{\begin{array}{r}-0.02 \\
0.20\end{array}\right.$ & $\begin{array}{c}a^{4} \mathrm{P}_{11 / 2}-v{ }^{4} \mathrm{D}_{11 / 2}^{\circ} \\
a^{4} \mathrm{H}_{61 / 2}-u{ }^{4} \mathrm{H}_{51 / 2}^{1}\end{array}$ & \\
\hline 3097. 063 & 30 & 32279.31 & 0.05 & $a^{4} \mathrm{P}_{21 / 2}^{0}-v^{4} \mathrm{D}_{21 / 2}^{0}$ & \\
\hline 3097. 758 & 5 & 32272.07 & -0.03 & $a^{4} \mathrm{H}_{51 / 2}-u^{4} \mathrm{G}_{41 / 2}^{0}$ & \\
\hline 3098. 092 & 10 & 32268.57 & -0.03 & $a^{4} \mathrm{P}_{21 / 2}-v^{4} \mathrm{D}_{31 / 2}^{\circ}$ & \\
\hline 3099. 301 & 1 & 32256.01 & -0.26 & $a^{4} \mathrm{H}_{41 / 2}^{272}-66600_{31 / 2}^{\circ}$ & \\
\hline 3100.267 & 8 & 32245.96 & -0.08 & $a{ }^{4} \mathrm{P}_{01 / 2}-v{ }^{4} \mathrm{D}_{11 / 2}^{\circ}$ & \\
\hline 3100.310 & 10 & 32245.51 & 0.00 & $a{ }^{4} \mathrm{P}_{01 / 2}-v{ }^{4} \mathrm{D}_{01 / 2}^{\circ}$ & \\
\hline 3101.529 & 30 & 32232.84 & 0.04 & $a^{4} \mathrm{P}_{11 / 2}-v{ }^{4} \mathrm{D}_{21 / 2}^{1}$ & 4 \\
\hline 3101. 836 & 2 & 32229.65 & -0.05 & $a^{4} \mathrm{H}_{41 / 2}-u{ }^{4} \mathrm{G}_{31 / 2}$ & \\
\hline 3103. 280 & 1 & 32214.65 & 0. 04 & $a{ }^{4} \mathrm{P}_{21 / 2}-x^{4} \mathrm{~F}_{11 / 2}^{0}$ & \\
\hline 3106. 336 & 2 & 32182.96 & 0.05 & $a^{4} \mathrm{P}_{21 / 2}-u{ }^{4} \mathrm{P}_{11 / 2}$ & \\
\hline 3106.748 & 10 & 32178.69 & -0.03 & $a^{4} \mathrm{H}_{41 / 2}-u{ }^{4} \mathrm{G}_{41 / 2}$ & 6 \\
\hline 3107.776 & 20 & 32168.05 & $\left\{\begin{array}{rl}-0 . & 10 \\
0.02 & 02\end{array}\right.$ & $\begin{array}{l}a{ }^{4} \mathrm{P}_{11 / 2}-x{ }^{4} \mathrm{~F}_{11 / 2}^{11} \\
a^{4} \mathrm{H}_{51}\end{array}$ & 6 \\
\hline 3108. 634 & 10 & 32159.17 & -0.01 & $a^{4} \mathrm{P}_{21 / 2}^{51 / 2}-x{ }^{4} \mathrm{~F}_{21 / 2}^{0}$ & 6 \\
\hline 3109.435 & $4 \mathrm{H} w$ & 32150.89 & -0.19 & $b{ }^{4} \mathrm{D}_{31 / 2}-v{ }^{4} \mathrm{~F}_{31 / 2}^{0}$ & \\
\hline 3110.681 & 50 & 32138. 01 & 0.00 & $a^{4} \mathrm{P}_{21 / 2}-w^{4} \mathrm{D}_{31 / 2}^{\circ}$ & 4 \\
\hline 3111. 147 & 2 & 32133. 20 & 0.05 & $b{ }^{4} \mathrm{D}_{31 / 2}-v{ }^{4} \mathrm{~F}_{21 / 2}^{0}$ & \\
\hline 3113.118 & 10 & 32112.85 & 0.13 & $a{ }^{4} \mathrm{P}_{11 / 2}-x{ }^{4} \mathrm{~F}_{21 / 2}^{0}$ & 4 \\
\hline 3113. 362 & 4 & 32110.33 & -0.04 & $a^{4} \mathrm{H}_{41 / 2}-u^{4} \mathrm{G}_{31 / 2}$ & 5 \\
\hline 3113.800 & & 32105.81 & -0.07 & $a{ }^{4} \mathrm{H}_{51 / 2}-u{ }^{4} \mathrm{H}_{41 / 2}^{0}$ & 5 \\
\hline 3114.115 & 3 & 32102.57 & -0.03 & $a^{4} \mathrm{P}_{01 / 2}-u{ }^{4} \mathrm{P}_{11 / 2}^{0}$ & \\
\hline 3114.440 & 1 & 32099. 22 & -0.13 & $a^{4} \mathrm{H}_{31 / 2}-u^{4} \mathrm{G}_{41 / 2}^{102}$ & \\
\hline 3115. 462 & 40 & 32088.69 & 0.00 & $a^{4} \mathrm{P}_{21 / 2}-x{ }^{4} \mathrm{~F}_{31 / 2}^{0}$ & 4 \\
\hline 3115. 754 & $3 \mathrm{H} w$ & 32085.68 & 0.00 & $b^{4} \mathrm{D}_{21 / 2}-v^{4} \mathrm{~F}_{31 / 2}^{\circ}$ & \\
\hline 3116.822 & & 32074.68 & 0.03 & $a{ }^{4} \mathrm{H}_{41 / 2}-u^{4} \mathrm{H}_{51 / 2}^{0}$ & \\
\hline 3117.505 & $3 \mathrm{H} w$ & 32067.66 & -0.09 & $b{ }^{4} \mathrm{D}_{21 / 2}-v{ }^{4} \mathrm{~F}_{21 / 2}^{0}$ & \\
\hline 3118. 099 & $8 \mathrm{H} w$ & 32061.55 & -0.10 & $b^{4} \mathrm{D}_{11 / 2}-v^{4} \mathrm{~F}_{21 / 2}^{2 / 2}$ & \\
\hline 3120.337 & $30 h$ & 32038.56 & -0.05 & $b{ }^{4} \mathrm{D}_{31 / 2}-v{ }^{4} \mathrm{~F}_{41 / 2}^{0}$ & 4 \\
\hline 3121. 072 & 6 & 32031.02 & 0. 02 & $a^{4} \mathrm{H}_{31 / 2}-u^{4} \mathrm{G}_{31 / 2}^{\circ}$ & 6 \\
\hline 3121. 914 & $1 h w$ & 32022.38 & 0. 3 & $z^{6} \mathrm{P}_{11 / 2}-h{ }^{8} \mathrm{D}$ & \\
\hline 3122. 880 & 10 & 32012.47 & -0.03 & $a^{4} \mathrm{H}_{41 / 2}-u{ }^{4} \mathrm{H}_{41 / 2}^{\circ}$ & 6 \\
\hline 3125.013 & 8 & 31990.62 & 0.05 & $a^{4} \mathrm{H}_{41 / 2}-u^{4} \mathrm{H}_{31 / 2}$ & 5 \\
\hline 3126. 192 & 2 & 31978.56 & 0.10 & $b{ }^{4} \mathrm{D}_{01 / 2}-v{ }^{4} \mathrm{~F}_{11 / 2}^{\circ}$ & \\
\hline 3126.846 & 10 & 31971.87 & -0.05 & $a{ }^{4} \mathrm{H}_{31 / 2}-u^{4} \mathrm{G}_{21 / 2}^{\circ}$ & 5 \\
\hline 3129.971 & 3 & 31939.95 & 0.02 & $a^{4} \mathrm{~F}_{31 / 2}-66981_{31 / 2}^{a}$ & \\
\hline 3130. 624 & 2 & 31933. 29 & 0.16 & $a^{4} \mathrm{H}_{31 / 2}-u^{4} \mathrm{H}_{41 / 2}^{\circ}$ & \\
\hline 3132. 284 & $15 h$ & 31916.36 & 0.06 & $a^{4} \mathrm{~F}_{41 / 2}-u^{4} \mathrm{~F}_{41 / 2}^{0}$ & $6,7 b$ \\
\hline 3132. 789 & 10 & 31911. 22 & 0. 02 & $a{ }^{4} \mathrm{H}_{31 / 2}-u{ }^{4} \mathrm{H}_{31 / 2}$ & 6 \\
\hline 3134. 922 & $4 h$ & 31889.51 & 0. 01 & $a^{4} \mathrm{D}_{31 / 2}-x^{4} \mathrm{D}_{21 / 2}^{\circ}$ & \\
\hline 3135.188 & 2 & 31886.80 & 0.06 & $z^{6} \mathrm{P}_{11 / 2}^{0}-i{ }^{6} \mathrm{D}_{01 / 2}$ & $7 b ?$ \\
\hline 3136. 958 & 10 & 31868.82 & 0. 22 & $a^{4} \mathrm{P}_{11 / 2}-u{ }^{4} \mathrm{P}_{21 / 2}^{o}$ & 4 \\
\hline 3138. 222 & 5 & 31855.98 & 0.04 & $a^{4} \mathrm{D}_{21 / 2}-w^{4} \mathrm{P}_{21 / 2}^{0}$ & \\
\hline 3141.555 & 1 & 31822.18 & -0.13 & $z^{6} \mathrm{P}_{11 / 2}^{0}-e{ }^{4} \mathrm{D}_{11 / 2}$ & \\
\hline 3141.821 & 5 & 31819.49 & 0.03 & $a^{4} \mathrm{D}_{21 / 2}-w^{4} \mathrm{P}_{11 / 2}^{\circ}$ & \\
\hline 3142. 401 & 1 & 31813.61 & $\left\{\begin{array}{r}-0.02 \\
0.03\end{array}\right.$ & $\begin{array}{l}a{ }^{4} \mathrm{~F}_{31 / 2}-u{ }^{4} \mathrm{~F}_{41 / 2}^{0} \\
z^{6} \mathrm{P}_{21 / 2}^{0}-e{ }^{4} \mathrm{D}_{11 / 2}^{0}\end{array}$ & \\
\hline
\end{tabular}


Table 1. Mn I-Classified lines-Continued

\begin{tabular}{|c|c|c|c|c|c|}
\hline 1 & 2 & 3 & 4 & 5 & 6 \\
\hline$\lambda$ & Int. & $\sigma$ & $\mathrm{o}-\mathrm{c}$ & Term designation & $\begin{array}{l}\text { Zeeman } \\
\text { type }\end{array}$ \\
\hline $\begin{array}{c}\AA \\
\text { Air } \\
3142.669 \\
3144.119 \\
3144.222 \\
3144.872 \\
3145.458\end{array}$ & $\begin{array}{l}20 w \\
1 \\
1 \\
3 h \\
2 h\end{array}$ & $\begin{array}{c}K \\
31810.90 \\
31776.23 \\
31795.19 \\
31788.62 \\
31782.69\end{array}$ & $\begin{array}{r}K \\
0.05 \\
-0.04 \\
0.15 \\
0.01 \\
0.06\end{array}$ & $\begin{array}{l}a^{4} \mathrm{D}_{31 / 2}-x^{4} \mathrm{D}_{31 / 2}^{\circ} \\
a^{4} \mathrm{~F}_{31 / 2}-u^{4} \mathrm{~F}_{21 / 2}^{\circ} \\
a^{4} \mathrm{~F}_{21 / 2}-66910_{21 / 2} \\
z^{6} \mathrm{P}_{11 / 2}^{\circ}-i{ }^{6} \mathrm{D}_{11 / 2} \\
z^{6} \mathrm{P}_{11 / 2}-e^{4} \mathrm{D}_{21 / 2}\end{array}$ & $6,7 b$ \\
\hline $\begin{array}{l}3145,727 \\
3146.324 \\
3148.182 \\
3148.857 \\
3149.529\end{array}$ & $\begin{array}{c}1 h \\
6 h \\
200 h \\
8 \\
6 h\end{array}$ & $\begin{array}{l}31779.98 \\
31773.95 \\
31755.20 \\
31748.39 \\
31741.62\end{array}$ & $\begin{array}{r}0.10 \\
0.05 \\
0.03 \\
-0.04 \\
-0.06\end{array}$ & $\begin{array}{c}z^{6} \mathrm{P}_{21 / 2}^{\circ}-i{ }^{6} \mathrm{D}_{11 / 2} \\
z^{6} \mathrm{P}_{21 / 2}^{o}-e^{4} \mathrm{D}_{21 / 2} \\
z^{8} \mathrm{P}_{21 / 2}^{o}-f^{8} \mathrm{~S}_{31 / 2} \\
a^{4} \mathrm{H}_{61 / 2}-v^{4} \mathrm{G}_{51 / 2}^{\circ} \\
a^{4} \mathrm{~F}_{31 / 2}-u^{4} \mathrm{~F}_{31 / 2}^{\circ}\end{array}$ & $\begin{array}{l}4 \\
4\end{array}$ \\
\hline $\begin{array}{l}3149.928 \\
3150.616 \\
3150.800 \\
3151.415 \\
3152.248\end{array}$ & $\begin{array}{c}5 \\
2 \\
1 \\
10 h \\
5\end{array}$ & $\begin{array}{l}31737.60 \\
31730.67 \\
31728.81 \\
31722.62 \\
31714.24\end{array}$ & $\begin{array}{r}-0.08 \\
-0.04 \\
0.00 \\
-0.04 \\
-0.02\end{array}$ & $\begin{array}{l}a{ }^{4} \mathrm{D}_{11 / 2}-w^{4} \mathrm{P}_{01 / 2}^{0} \\
a^{4} \mathrm{D}_{21 / 2}-x^{4} \mathrm{D}_{11 / 2}^{0} \\
a^{4} \mathrm{~F}_{21 / 2}-u{ }^{4} \mathrm{~F}_{11 / 2}^{11} \\
a^{4} \mathrm{~F}_{21 / 2}-u^{4} \mathrm{~F}_{21 / 2}^{1} \\
a^{2} \mathrm{H}_{51 / 2}-v^{2} \mathrm{H}_{51 / 2}^{0}\end{array}$ & $6,7 b$ \\
\hline $\begin{array}{l}\text { 3152. } 517 \\
3153.393 \\
3155.094 \\
3155.781 \\
3156.245\end{array}$ & $\begin{array}{l}4 \mathrm{H} w \\
7 \mathrm{H} w \\
1 \\
10 \\
2 h\end{array}$ & $\begin{array}{l}31711.53 \\
31702.72 \\
31685.63 \\
31678.73 \\
31674.07\end{array}$ & $\begin{array}{r}0.06 \\
-0.02 \\
0.01 \\
-0.01 \\
0.04\end{array}$ & $\begin{array}{l}z^{6} \mathrm{P}_{11 / 2}^{\circ}-i{ }^{6} \mathrm{D}_{21 / 2} \\
z^{6} \mathrm{P}_{21 / 2}-i{ }^{6} \mathrm{D}_{21 / 2} \\
a^{4} \mathrm{D}_{11 / 2}-w^{4} \mathrm{P}_{21 / 2}^{\circ} \\
a^{4} \mathrm{~F}_{11 / 2}-u{ }^{4} \mathrm{~F}_{11 / 2}^{2} \\
z^{6} \mathrm{P}_{21 / 2}^{\circ}-e{ }^{4} \mathrm{D}_{31 / 2}^{0}\end{array}$ & $6,7 b$ \\
\hline $\begin{array}{l}3156.392 \\
3157.658 \\
3157.811 \\
3158.723 \\
3159.825\end{array}$ & $\begin{array}{c}1 h \\
3 h \\
3 \\
10 \\
4\end{array}$ & $\begin{array}{l}31672.60 \\
31659.90 \\
31658.37 \\
31649.12 \\
31638.19\end{array}$ & $\begin{array}{r}0.01 \\
0.07 \\
-0.03 \\
-0.02 \\
-0.14\end{array}$ & $\begin{array}{c}a^{4} \mathrm{~F}_{11 / 2}-u{ }^{4} \mathrm{~F}_{21 / 2}^{\circ} \\
z^{6} \mathrm{P}_{31 / 2}^{\circ}-e^{4} \mathrm{D}_{31 / 2}^{\circ} \\
a^{4} \mathrm{H}_{51 / 2}-v^{4} \mathrm{G}_{41 / 2}^{\circ} \\
a^{4} \mathrm{D}_{11 / 2}-w^{4} \mathrm{P}_{11 / 2}^{\circ} \\
a^{4} \mathrm{D}_{01 / 2}-w^{4} \mathrm{P}_{01 / 2}^{\circ}\end{array}$ & 6 \\
\hline $\begin{array}{l}3159.952 \\
3160.155 \\
3161.050 \\
3162.210 \\
3163.365\end{array}$ & $\begin{array}{c}20 \\
6 \\
200 h \\
1 h \\
1\end{array}$ & $\begin{array}{l}31636.92 \\
31634.89 \\
31625.93 \\
31614.33 \\
31602.79\end{array}$ & $\begin{array}{r}-0.05 \\
-0.01 \\
-0.06 \\
-0.10 \\
0.01\end{array}$ & $\begin{array}{l}a{ }^{4} \mathrm{D}_{21 / 2}-x^{4} \mathrm{D}_{21 / 2}^{\circ} \\
a^{4} \mathrm{~F}_{41 / 2}-u^{4} \mathrm{G}_{51 / 2}^{1} \\
z^{8} \mathrm{P}_{31 / 2}^{\circ}-f^{8} \mathrm{~S}_{31 / 2} \\
b^{4} \mathrm{D}_{11 / 2}-y^{2} \mathrm{~F}_{31 / 2}^{\circ} \\
a^{2} \mathrm{H}_{41 / 2}^{2}-v^{2} \mathrm{H}_{51 / 2}^{\circ 1}\end{array}$ & $\begin{array}{c}6,7 b \\
4 \\
6\end{array}$ \\
\hline $\begin{array}{l}3163.534 \\
3165.253 \\
3166.827 \\
3167.153 \\
3167.619\end{array}$ & $\begin{array}{c}1 \\
1 \\
10 h \\
2 \\
3\end{array}$ & $\begin{array}{l}31601.10 \\
31583.94 \\
31568.24 \\
31564.99 \\
31560.35\end{array}$ & $\begin{array}{r}-0.3 \\
0.02 \\
0.08 \\
-0.03 \\
-0.04\end{array}$ & $\begin{array}{l}a^{4} \mathrm{G}_{51 / 2}-u^{6} \mathrm{~F}^{\circ} \\
a^{4} \mathrm{~F}_{41 / 2}-u{ }^{4} \mathrm{G}_{41 / 2}^{\circ} \\
z^{6} \mathrm{P}_{21 / 2}^{\circ}-i^{6} \mathrm{D}_{31 / 2}^{\circ} \\
a^{4} \mathrm{H}_{41 / 2}-v{ }^{4} \mathrm{G}_{41 / 2}^{\circ} \\
a^{4} \mathrm{D}_{11 / 2}-x{ }^{4} \mathrm{D}_{11 / 2}^{\circ}\end{array}$ & \\
\hline $\begin{array}{l}3167.827 \\
3167.935 \\
3168.254 \\
3168.680 \\
3169.356\end{array}$ & $\begin{array}{l}8 h \\
1 \\
4 h \\
2 \\
4\end{array}$ & $\begin{array}{l}31558.28 \\
31557.20 \\
31554.02 \\
31549.78 \\
31543.06\end{array}$ & $\begin{array}{r}-0.04 \\
-0.06 \\
0.06 \\
-0.01 \\
0.04\end{array}$ & $\begin{array}{l}a{ }^{4} \mathrm{D}_{21 / 2}-x{ }^{4} \mathrm{D}_{31 / 2}^{\circ} \\
b^{4} \mathrm{P}_{11 / 2}-u{ }^{2} \mathrm{~F}_{21 / 2}^{21 / 2} \\
z^{6} \mathrm{P}_{31 / 2}^{\circ}-i{ }^{6} \mathrm{D}_{31 / 2} \\
a^{4} \mathrm{D}_{01 / 2}-w^{4} \mathrm{P}_{11 / 2}^{\circ} \\
a^{2} \mathrm{H}_{41 / 2}-v^{2} \mathrm{H}_{41 / 2}^{\circ}\end{array}$ & 6,7 \\
\hline $\begin{array}{l}3170.427 \\
3173.845 \\
3174.746 \\
3175.355 \\
3175.576\end{array}$ & $\begin{array}{l}4 \\
3 \\
20 \mathrm{H} w \\
3 h \\
12\end{array}$ & $\begin{array}{l}31532.40 \\
31498.44 \\
31489.50 \\
31483.47 \\
31481.28\end{array}$ & $\begin{array}{r}-0.04 \\
-0.09 \\
-0.10 \\
-0.03 \\
0.03\end{array}$ & $\begin{array}{l}a{ }^{4} \mathrm{H}_{41 / 2}-v^{4} \mathrm{G}_{31 / 2}^{\circ} \\
b^{4} \mathrm{P}_{11 / 2}-w^{2} \mathrm{D}_{11 / 2}^{\circ} \\
a^{4} \mathbf{F}_{11 / 2}-66654_{21 / 2}^{1} \\
b^{4} \mathbf{P}_{11 / 2}-w^{2} \mathrm{D}_{21 / 2}^{\circ} \\
a^{4} \mathbf{F}_{31 / 2}-u^{4} \mathrm{G}_{41 / 2}^{0}\end{array}$ & 4 \\
\hline $\begin{array}{l}3175.713 \\
3177.044 \\
3177.616 \\
3178.501 \\
3178.730\end{array}$ & $\begin{array}{r}10 \\
10 \\
5 \\
200 \\
2\end{array}$ & $\begin{array}{l}31479.91 \\
31466.72 \\
31461.06 \\
31452.30 \\
31450.03\end{array}$ & $\begin{array}{r}0.06 \\
0.07 \\
0.02 \\
0.04 \\
-0.10\end{array}$ & $\begin{array}{c}a^{4} \mathrm{~F}_{41 / 2}-u^{4} \mathrm{H}_{51 / 2}^{\circ} \\
a^{4} \mathrm{D}_{11 / 2}-x{ }^{4} \mathrm{D}_{21 / 2}^{\circ} \\
a^{4} \mathrm{D}_{01 / 2}-x^{4} \mathrm{D}_{11 / 2}^{\circ} \\
z^{8} \mathrm{P}_{41 / 2}^{\circ}-f^{8} \mathrm{~S}_{31 / 2}^{\circ} \\
a^{4} \mathrm{H}_{31 / 2}-v^{4} \mathrm{G}_{21 / 2}^{\circ}\end{array}$ & $\begin{array}{l}5 \\
5 \\
4\end{array}$ \\
\hline
\end{tabular}


Table 1. Mn I-Classified lines-Continued

\begin{tabular}{|c|c|c|c|c|c|}
\hline 1 & 2 & 3 & 4 & 5 & 6 \\
\hline$\lambda$ & Int. & $\sigma$ & $\mathrm{o}-\mathrm{c}$ & Term designation & $\begin{array}{l}\text { Zeeman } \\
\text { type }\end{array}$ \\
\hline $\begin{array}{c}\AA \\
\text { Air } \\
3181.269 \\
3181.990 \\
3182.498 \\
3185.096 \\
3186.507\end{array}$ & $\begin{array}{c}1 \\
1 \\
2 \\
10 h \\
1 h\end{array}$ & $\begin{array}{c}K \\
31424.94 \\
31417.82 \\
31412.90 \\
31387.18 \\
31373.28\end{array}$ & $\begin{array}{r}K \\
0.03 \\
0.12 \\
0.00 \\
-0.02 \\
0.03\end{array}$ & $\begin{array}{l}a{ }^{4} \mathrm{H}_{41 / 2}-65768^{\circ}{ }^{11 / 2} \\
a{ }^{4} \mathrm{~F}_{41 / 2}-u{ }^{4} \mathrm{H}_{41 / 2}^{\circ} \\
a \\
{ }^{4} \mathrm{~F}_{31 / 2}-u{ }^{4} \mathrm{G}_{31 / 2}^{\circ} \\
z^{6} \mathrm{P}_{31 / 2}^{\circ}-i{ }^{6} \mathrm{D}_{41 / 2} \\
b^{4} \mathrm{D}_{31 / 2}-z^{2} \mathrm{~F}_{21 / 2}^{2}\end{array}$ & \\
\hline $\begin{array}{l}\text { 3187. } 213 \\
3189.959 \\
3192.242 \\
3193.788 \\
3194.663\end{array}$ & $\begin{array}{r}2 \\
15 \\
10 \\
1 \\
1 h\end{array}$ & $\begin{array}{l}\text { 31366. } 33 \\
31339.33 \\
31315.11 \\
31301.76 \\
31293.19\end{array}$ & $\begin{array}{l}0.00 \\
0.04 \\
0.08 \\
0.01 \\
0.09\end{array}$ & $\begin{array}{l}b{ }^{4} \mathrm{D}_{21 / 2}-y{ }^{2} \mathrm{G}_{31 / 2}^{0} \\
a^{4} \mathrm{~F}_{21 / 2}-u{ }^{4} \mathrm{G}_{31 / 2}^{01} \\
a^{4} \mathrm{~F}_{31 / 2}-u{ }^{4} \mathrm{H}_{41 / 2}^{0} \\
b^{4} \mathrm{D}_{11 / 2}-z^{2} \mathrm{~F}_{21 / 2}^{0} \\
a^{4} \mathrm{~F}_{31 / 2}-u{ }^{4} \mathrm{H}_{31 / 2}^{0}\end{array}$ & 4 \\
\hline $\begin{array}{l}3194.855 \\
3195.988 \\
3201.113 \\
3202.205 \\
3203.131\end{array}$ & $\begin{array}{r}1 h \\
2 \\
10 \\
4 \\
4\end{array}$ & $\begin{array}{l}31291.31 \\
31280.22 \\
31230.14 \\
31219.49 \\
31210.47\end{array}$ & $\begin{array}{r}-0.06 \\
0.01 \\
0.00 \\
0.00 \\
0.07\end{array}$ & 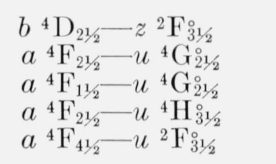 & $\tilde{5}$ \\
\hline $\begin{array}{l}\text { 3206. } 910 \\
3211.270 \\
3212.887 \\
3216.947 \\
3224.758\end{array}$ & $\begin{array}{l}80 \\
\quad 4 h \\
200 \\
100 r \\
150 \mathrm{R}\end{array}$ & $\begin{array}{l}31173.69 \\
31131.36 \\
31115.70 \\
31076.42 \\
31001.15\end{array}$ & $\begin{array}{r}-0.01 \\
0.23 \\
-0.02 \\
0.00 \\
0.00\end{array}$ & 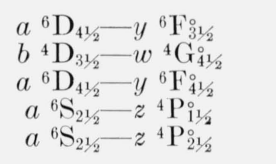 & $\begin{array}{l}5 \\
6 \\
5 \\
6\end{array}$ \\
\hline $\begin{array}{l}3226.048 \\
3227.039 \\
3228.092 \\
3230.231 \\
3230.716\end{array}$ & $\begin{array}{c}100 \\
2 \\
1000 \mathrm{R} \\
10 \\
500 \mathrm{R}\end{array}$ & $\begin{array}{l}\text { 30988. } 76 \\
\text { 30979. } 24 \\
\text { 30969. } 14 \\
\text { 30948. } 64 \\
\text { 30943. } 98\end{array}$ & $\begin{array}{r}-0.15 \\
-0.02 \\
0.00 \\
0.03 \\
-0.01\end{array}$ & 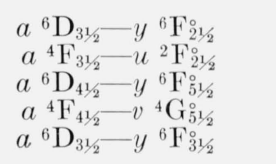 & $\begin{array}{l}4 \\
5\end{array}$ \\
\hline $\begin{array}{l}\text { 3233. } 939 \\
3233.997 \\
3234.112 \\
3234.737 \\
3234.912\end{array}$ & $\begin{array}{c}200 \mathrm{H} \\
4 \\
10 h\end{array}$ & $\left\{\begin{array}{r}30913.12 \\
30912.67 \\
30911.46 \\
30905.53 \\
30903.82\end{array}\right.$ & $\begin{array}{r}0.04 \\
0.09 \\
-0.02 \\
0.03 \\
-0.03\end{array}$ & $\begin{array}{l}z^{6} \mathrm{P}_{11 / 2}^{o}-h{ }^{6} \mathrm{D}_{01 / 2} \\
z^{6} \mathrm{P}_{11 / 2}^{o}-h{ }^{6} \mathrm{D}_{11 / 2} \\
z^{6} \mathrm{P}_{11 / 2}^{o}-h{ }^{6} \mathrm{D}_{21 / 2} \\
a^{4} \mathrm{~F}_{31 / 2}-w^{2} \mathrm{D}_{21 / 2}^{12} \\
z^{6} \mathrm{P}_{21 / 2}^{o}-h{ }^{6} \mathrm{D}_{11 / 2}\end{array}$ & 4 \\
\hline $\begin{array}{l}3235.025 \\
3235.307 \\
3236.515\end{array}$ & $\begin{array}{r}200 h \\
300 h \\
5 h\end{array}$ & $\begin{array}{l}\text { 30902. } 74 \\
30900.05 \\
30888.55\end{array}$ & $\begin{array}{r}-0.01 \\
0.00 \\
0.00\end{array}$ & $\begin{array}{l}z^{6} \mathrm{P}_{21 / 2}^{\circ}-h{ }^{6} \mathrm{D}_{21 / 2} \\
z^{6} \mathrm{P}_{21 / 2}^{o}-h{ }^{6} \mathrm{D}_{31 / 2} \\
z^{6} \mathrm{P}_{31 / 2}^{o}-h{ }^{6} \mathrm{D}_{21 / 2}\end{array}$ & $\begin{array}{l}\mathrm{P}-\mathrm{B} \\
\mathrm{P}-\mathrm{B}\end{array}$ \\
\hline $\begin{array}{l}3236.778 \\
3237.019\end{array}$ & $\begin{array}{r}1000 \\
1\end{array}$ & $\begin{array}{l}\text { 30886. } 04 \\
\text { 30883. } 74\end{array}$ & $\left\{\begin{array}{l}0.03 \\
0.19 \\
0.03\end{array}\right.$ & $\begin{array}{c}a^{6}{ }^{6} \mathrm{D}_{31 / 2}-y^{6} \mathrm{~F}_{41 / 2} \\
\left(z^{6} \mathrm{P}^{0}{ }_{31 / 2}-h{ }^{6} \mathrm{D}_{31 / 2}\right) \\
z^{8} \mathrm{P}_{31 / 2}^{0}-f^{6} \mathrm{~S}_{21 / 2}\end{array}$ & 4 \\
\hline $\begin{array}{l}3237.443 \\
3238.720 \\
3240.408 \\
3240.613 \\
3240.882\end{array}$ & $\begin{array}{c}500 \mathrm{H} w \\
5 \\
150 \\
100 \\
6\end{array}$ & $\begin{array}{l}30879.67 \\
30867.51 \\
30851.52 \\
30849.49 \\
30846.93\end{array}$ & $\begin{array}{r}0.02 \\
-0.04 \\
0.01 \\
0.03 \\
0.01\end{array}$ & $\begin{array}{l}z^{6} \mathrm{P}_{31 / 2}^{\circ}-h{ }^{6} \mathrm{D}_{41 / 2} \\
a^{4} \mathrm{~F}_{31 / 2}-v{ }^{4} \mathrm{G}_{41 / 2}^{0} \\
a^{6} \mathrm{D}_{41 / 2}-y{ }^{6} \mathrm{D}_{41 / 2}^{\circ} \\
a^{6} \mathrm{D}_{21 / 2}-y \\
{ }^{6} \mathrm{~F}_{11 / 2}^{0} \\
a^{4} \mathrm{~F}_{21 / 2}-w^{2} \mathrm{D}_{11 / 2}^{0}\end{array}$ & $\begin{array}{r}\mathrm{P}-\mathrm{B} \\
4 \\
6, \underset{7 b}{\mathrm{P}}-\mathrm{B}\end{array}$ \\
\hline $\begin{array}{l}3242.139 \\
3242.463 \\
3243.777 \\
3246.153 \\
3248.512\end{array}$ & $\begin{array}{c}1 \\
2 h \\
500 \\
3 \\
700 r\end{array}$ & $\begin{array}{l}\text { 30834. } 97 \\
30831.89 \\
30819.40 \\
30796.84 \\
30774.48\end{array}$ & $\begin{array}{r}0.00 \\
0.00 \\
0.01 \\
-0.01 \\
0.01\end{array}$ & $\begin{array}{c}a^{4} \mathrm{~F}_{31 / 2}-v{ }^{4} \mathrm{G}_{31 / 2}^{\circ} \\
a^{4} \mathrm{~F}_{21 / 2}-w^{2} \mathrm{D}_{21 / 2} \\
a^{6} \mathrm{D}_{21 / 2}-y^{6} \mathrm{~F}_{21 / 2}^{\circ} \\
a^{4} \mathrm{~F}_{11 / 2}-w^{2} \mathrm{D}_{11 / 2}^{\circ} \\
a^{6} \mathrm{D}_{21 / 2}-y \\
{ }^{6} \mathrm{~F}_{31 / 2}^{0}\end{array}$ & 4 \\
\hline $\begin{array}{l}3249.894 \\
3251.134 \\
3252.949 \\
3254.037 \\
3255.508\end{array}$ & $\begin{array}{c}6 \\
150 \\
500 r \\
100 \\
5\end{array}$ & $\begin{array}{l}30761.40 \\
30749.66 \\
30732.50 \\
30722.23 \\
30708.35\end{array}$ & $\begin{array}{l}0.04 \\
0.02 \\
0.00 \\
0.00 \\
0.00\end{array}$ & 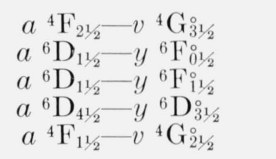 & $\begin{array}{r}\mathrm{P}-\mathrm{B} \\
5 \\
6 \\
4 \\
\mathrm{P}-\mathrm{B}\end{array}$ \\
\hline $\begin{array}{l}3256.137 \\
3258.414 \\
3260.238 \\
3262.333 \\
3263.037\end{array}$ & $\begin{array}{c}500 r \\
400 r \\
300 r \\
3 \\
2\end{array}$ & $\begin{array}{l}30702.42 \\
30680.96 \\
30663.80 \\
30644.10 \\
30637.49\end{array}$ & $\begin{array}{r}-0.01 \\
-0.01 \\
-0.03 \\
-0.22 \\
0.01\end{array}$ & $\begin{array}{l}a^{6} \mathrm{D}_{11 / 2}-y{ }^{6} \mathrm{~F}_{21 / 2}^{\circ} \\
a^{6} \mathrm{D}_{01 / 2}-y^{6} \mathrm{~F}_{01 / 2}^{\circ} \\
a^{6} \mathrm{D}_{01 / 2}-y{ }^{6} \mathrm{~F}_{11 / 2}^{112} \\
a^{4} \mathrm{H}_{41 / 2}-w^{2} \mathrm{~F}_{31 / 2}^{112} \\
a^{4} \mathrm{H}_{51 / 2}-v^{4} \mathrm{H}_{41 / 2}{ }_{41 / 2}^{\circ}\end{array}$ & $\begin{array}{l}4 \\
6 \\
4\end{array}$ \\
\hline
\end{tabular}


Table 1. Mn I-Classified lines-Continued

\begin{tabular}{|c|c|c|c|c|c|}
\hline 1 & 2 & 3 & 4 & 5 & 6 \\
\hline$\lambda$ & Int. & $\sigma$ & $\mathrm{o}-\mathrm{c}$ & Term designation & $\begin{array}{l}\text { Zeeman } \\
\text { type }\end{array}$ \\
\hline $\begin{array}{c}\stackrel{\AA}{\mathrm{A}} \\
\text { Air } \\
3264.710 \\
3267.789 \\
3268.720 \\
3270.353 \\
3270.781\end{array}$ & $\begin{array}{c}300 \\
80 \\
70 \\
60 \\
5 h\end{array}$ & $\begin{array}{c}K \\
\text { 30621. } 79 \\
30592.94 \\
30584.23 \\
30568.96 \\
30564.96\end{array}$ & $\begin{array}{r}K \\
-0.01 \\
-0.06 \\
0.03 \\
-0.05 \\
0.01\end{array}$ & 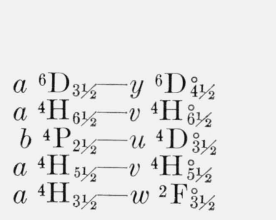 & $\begin{array}{ll}6, & 4 \\
6 b \\
6, & 7 b\end{array}$ \\
\hline $\begin{array}{l}3273.016 \\
3278.062 \\
3278.551 \\
3279.751 \\
3280.370\end{array}$ & $\begin{array}{c}50 \\
15 h \\
100 \\
5 \\
5\end{array}$ & $\begin{array}{l}30544.09 \\
30497.07 \\
30492.52 \\
30481.37 \\
30475.62\end{array}$ & $\begin{array}{r}-0.01 \\
0.01 \\
0.00 \\
0.01 \\
-0.01\end{array}$ & 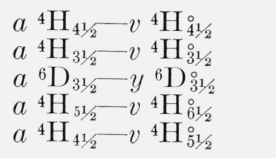 & $\begin{array}{l}6,7 b \\
6,7 b\end{array}$ \\
\hline $\begin{array}{l}3280.763 \\
3281.415 \\
3281.532 \\
3285.482 \\
3288.548\end{array}$ & $\begin{array}{c}100 \\
1 \\
2 \\
1 h \\
1 \mathrm{H}\end{array}$ & $\begin{array}{l}30471.96 \\
30465.91 \\
30464.82 \\
30428.20 \\
30399.83\end{array}$ & $\begin{array}{r}-0.03 \\
-0.23 \\
0.09 \\
0.02 \\
-0.11\end{array}$ & $\begin{array}{c}a{ }^{6} \mathrm{D}_{31 / 2}-y^{6} \mathrm{D}_{21 / 2}^{\circ} \\
b^{4} \mathrm{D}_{31 / 2}-w^{4} \mathrm{~F}_{21 / 2}^{0} \\
a^{4} \mathrm{H}_{31 / 2}-v^{4} \mathrm{H}_{41 / 2}^{112} \\
a^{2} \mathrm{I}_{51 / 2}-w^{2} \mathrm{H}_{51 / 2}^{0} \\
a^{4} \mathrm{H}_{31 / 2}-w^{2} \mathrm{~F}_{21 / 2}^{0}\end{array}$ & \\
\hline $\begin{array}{l}3288.644 \\
3289.106 \\
3290.969 \\
3294.030 \\
3294.934\end{array}$ & $\begin{array}{l}2 \mathrm{H} \\
1 \\
25 \\
1 h ? \\
1 h\end{array}$ & $\begin{array}{l}30398.94 \\
30394.67 \\
30377.46 \\
30349.24 \\
30340.91\end{array}$ & $\begin{array}{r}0.01 \\
0.03 \\
-0.06 \\
0.11 \\
-0.35\end{array}$ & $\begin{array}{l}b^{4} \mathrm{G}_{51 / 2}-t^{4} \mathrm{G}_{41 / 2}^{0} \\
b^{4} \mathrm{D}_{11 / 2}-w^{4} \mathrm{~F}_{21 / 2}^{\circ} \\
a^{6} \mathrm{D}_{31 / 2}-w^{6} \mathrm{P}_{21 / 2}^{0} \\
b^{4} \mathrm{D}_{01 / 2}-w^{4} \mathrm{~F}_{11 / 2}^{\circ} \\
b^{4} \mathrm{D}_{21 / 2}-w^{4} \mathrm{~F}_{11 / 2}^{0}\end{array}$ & 4 \\
\hline $\begin{array}{l}3295.840 \\
3296.025 \\
3296.879 \\
3298.228 \\
3300.943\end{array}$ & $\begin{array}{r}20 \\
40 \\
150 \\
120 \\
10\end{array}$ & $\begin{array}{l}30332.57 \\
30330.88 \\
30323.01 \\
30310.61 \\
30285.68\end{array}$ & $\begin{array}{r}-0.03 \\
-0.03 \\
0.01 \\
-0.01 \\
0.14\end{array}$ & $\begin{array}{c}b^{4} \mathrm{G}_{51 / 2}-t^{4} \mathrm{G}_{51 / 2}^{0} \\
a^{6} \mathrm{D}_{21 / 2}-w^{6} \mathrm{P}_{11 / 2} \\
a^{6} \mathrm{D}_{21 / 2}-y{ }^{6} \mathrm{D}_{31 / 2}^{0} \\
a^{4} \mathrm{P}_{21 / 2}-y{ }^{4} \mathrm{~S}_{11 / 2}^{0} \\
a^{4} \mathrm{P}_{21 / 2}-v{ }^{4} \mathrm{P}_{21 / 2}^{0}\end{array}$ & $\begin{array}{ll}6, & 7 b \\
& 4 \\
& 4 \\
& 4 \\
6, & 7 b\end{array}$ \\
\hline $\begin{array}{l}\text { 3303. } 280 \\
3303.681 \\
3304.898 \\
3306.004 \\
3306.998\end{array}$ & $\begin{array}{c}100 \\
1 h \\
15 h \\
2 \\
40\end{array}$ & $\begin{array}{l}30264.25 \\
30260.58 \\
30249.44 \\
30239.32 \\
30230.23\end{array}$ & $\begin{array}{r}0.09 \\
-0.16 \\
-0.13 \\
0.24 \\
-0.08\end{array}$ & $\begin{array}{l}a^{4} \mathrm{P}_{11 / 2}-y{ }^{4} \mathrm{~S}_{11 / 2}^{0} \\
b^{4} \mathrm{G}_{41 / 2}-t^{4} \mathrm{G}_{31 / 2}^{\circ} \\
b^{4} \mathrm{P}_{11 / 2}-u{ }^{4} \mathrm{D}_{21 / 2}^{0} \\
a^{4} \mathrm{P}_{11 / 2}-v \\
{ }^{4} \mathrm{P}_{21 / 2}^{0} \\
a^{4} \mathrm{P}_{01 / 2}-y{ }^{4} \mathrm{~S}_{11 / 2}^{0}\end{array}$ & 4 \\
\hline $\begin{array}{l}\text { 3308. } 065 \\
3308.778 \\
3309.428 \\
3311.586 \\
3311.895\end{array}$ & $\begin{array}{c}8 h \\
40 \\
2 \\
6 h w \\
100 l\end{array}$ & $\begin{array}{l}30220.48 \\
30213.97 \\
30208.04 \\
30188.35 \\
30185.54\end{array}$ & $\begin{array}{r}-0.10 \\
0.02 \\
0.04 \\
-0.20 \\
0.03\end{array}$ & $\begin{array}{c}b^{4} \mathrm{P}_{11 / 2}-u^{4} \mathrm{D}_{11 / 2}^{\circ} \\
a^{6} \mathrm{D}_{11 / 2}-w^{6} \mathrm{P}_{11 / 2}^{\circ} \\
a^{6} \mathrm{D}_{21 / 2}-w^{6} \mathrm{P}_{21 / 2}^{\circ} \\
b^{4} \mathrm{G}_{41 / 2}-t^{4} \mathrm{G}_{41 / 2}^{\circ} \\
a^{6} \mathrm{D}_{11 / 2}-y^{6} \mathrm{D}_{21 / 2}^{0}\end{array}$ & $\begin{array}{l}6 \\
6\end{array}$ \\
\hline $\begin{array}{l}3313.050 \\
3313.200 \\
3313.458 \\
3313.560 \\
3314.146\end{array}$ & $\begin{array}{l}1 \mathrm{H} ? \\
50 h l \\
10 h \\
40 h \\
10 h l\end{array}$ & $\begin{array}{l}30175.01 \\
30173.65 \\
30171.30 \\
30170.36 \\
30165.03\end{array}$ & $\begin{array}{r}0.07 \\
-0.05 \\
-0.04 \\
-0.22 \\
0.06\end{array}$ & 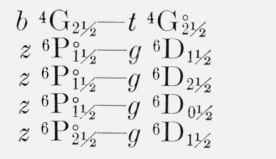 & $\begin{array}{l}\mathrm{P}-\mathrm{B} \\
\mathrm{P}-\mathrm{B} \\
\mathrm{P}-\mathrm{B}\end{array}$ \\
\hline $\begin{array}{l}3314.415 \\
3314.889 \\
3315.343 \\
3315.975 \\
3316.319\end{array}$ & $\begin{array}{c}50 h l \\
100 h l \\
4 h \\
7 h l \\
20\end{array}$ & $\begin{array}{l}30162.59 \\
30158.27 \\
30154.14 \\
30148.40 \\
30145.27\end{array}$ & $\begin{array}{r}-0.02 \\
0.23 \\
0.00 \\
-0.01 \\
-0.01\end{array}$ & $\begin{array}{l}z^{6} \mathrm{P}_{21 / 2}^{\circ}-g{ }^{6} \mathrm{D}_{21 / 2} \\
z^{6} \mathrm{P}_{21 / 2}^{0}-g{ }^{6} \mathrm{D}_{31 / 2}^{1} \\
b^{4} \mathrm{G}_{31 / 2}-t^{4} \mathrm{G}_{31 / 2}^{1} \\
z^{6} \mathrm{P}_{31 / 2}^{0}-g{ }^{6} \mathrm{D}_{21 / 2} \\
a^{6} \mathrm{D}_{01 / 2}-w^{6} \mathrm{P}_{11 / 2}^{1}\end{array}$ & $\begin{array}{l}\mathrm{P}-\mathrm{B} \\
\mathrm{P}-\mathrm{B}\end{array}$ \\
\hline $\begin{array}{l}3316.459 \\
3317.289 \\
3318.874 \\
3319.873 \\
3320.692\end{array}$ & $\begin{array}{c}60 h l \\
200 h l \\
1 h \\
3 \\
100\end{array}$ & $\begin{array}{l}30144.00 \\
30136.46 \\
30122.08 \\
30113.00 \\
30105.57\end{array}$ & $\begin{array}{r}0.16 \\
0.15 \\
-0.14 \\
0.22 \\
-0.05\end{array}$ & 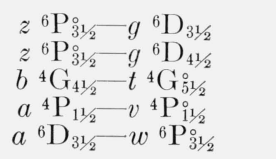 & $\begin{array}{l}\mathrm{P}-\mathrm{B} \\
\mathrm{P}-\mathrm{B}\end{array}$ \\
\hline $\begin{array}{l}3322.295 \\
3323.633 \\
3330.663 \\
3334.557 \\
3335.039\end{array}$ & $\begin{array}{c}6 \\
6 \\
100 c \\
8 \\
3\end{array}$ & $\begin{array}{l}30091.05 \\
30078.93 \\
30015.45 \\
29980.40 \\
29976.06\end{array}$ & $\begin{array}{l}0.01 \\
0.00 \\
0.31 \\
0.10 \\
0.03\end{array}$ & 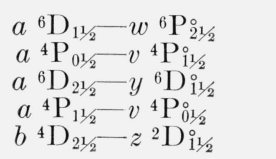 & 4 \\
\hline
\end{tabular}


Table 1. Mn I-Classified lines-Continued

\begin{tabular}{|c|c|c|c|c|c|}
\hline 1 & 2 & 3 & 4 & 5 & 6 \\
\hline$\lambda$ & Int. & $\sigma$ & $\mathrm{o}-\mathrm{c}$ & Term designation & $\begin{array}{l}\text { Zeeman } \\
\text { type }\end{array}$ \\
\hline $\begin{array}{c}\text { A } \\
\text { jAir } \\
\text { 3338. } 002 \\
3338.288 \\
3338.346 \\
3339.126 \\
3339.486\end{array}$ & $\begin{array}{l}1 \\
1 h \\
2 h \\
2 h \\
1 h\end{array}$ & $\begin{array}{c}K \\
\text { 29949. } 46 \\
29946.90 \\
29946.37 \\
29939.38 \\
29936.15\end{array}$ & $\begin{array}{r}K \\
0.16 \\
0.05 \\
-0.08 \\
-0.03 \\
0.05\end{array}$ & $\begin{array}{c}a^{4} \mathrm{~F}_{41 / 2}-v^{4} \mathrm{H}_{41 / 2}^{\circ} \\
a^{4} \mathrm{~F}_{31 / 2}-w^{2} \mathrm{~F}_{31 / 2}^{0} \\
a^{4} \mathrm{P}_{01 / 2}-v^{4} \mathrm{P}_{01 / 2}^{\circ} \\
b^{4} \mathrm{P}_{21 / 2}-x^{2} \mathrm{D}_{21 / 2}^{\circ} \\
a^{6} \mathrm{D}_{21 / 2}-w^{6} \mathrm{P}_{31 / 2}^{0}\end{array}$ & \\
\hline $\begin{array}{l}\text { 3342. } 065 \\
\text { 3343. } 722 \\
3345.349 \\
3345.656 \\
3349.494\end{array}$ & $\begin{array}{r}4 \\
60 \\
50 \\
2 \\
2\end{array}$ & $\begin{array}{l}29913.05 \\
29898.23 \\
29883.68 \\
29880.94 \\
29846.71\end{array}$ & $\begin{array}{l}0.02 \\
0.05 \\
0.00 \\
0.11 \\
0.08\end{array}$ & 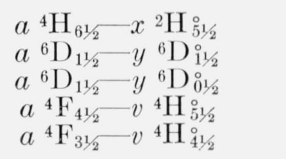 & 4 \\
\hline $\begin{array}{l}3350.409 \\
3351.423 \\
3351.656 \\
3353.054 \\
3353.328\end{array}$ & $\begin{array}{l}10 h \\
10 \\
20 c \\
1 \\
4 h\end{array}$ & $\begin{array}{l}29838.56 \\
29829.53 \\
29827.46 \\
29815.02 \\
29812.58\end{array}$ & $\begin{array}{r}-0.13 \\
0.02 \\
0.04 \\
0.01 \\
0.04\end{array}$ & $\begin{array}{c}b^{4} \mathrm{P}_{01 / 2}-u^{4} \mathrm{D}_{11 / 2}^{\circ} \\
a^{6} \mathrm{D}_{01 / 2}-y{ }^{6} \mathrm{D}_{11 / 2}^{1} \\
a^{4} \mathrm{D}_{31 / 2}-y \\
a^{4} \mathrm{D}_{31 / 2}^{\circ} \\
a^{6} \mathrm{D}_{01 / 2}-y \\
{ }^{6} \mathrm{D}_{01 / 2}^{\circ} \\
a^{4} \mathrm{D}_{31 / 2} y{ }^{4} \mathrm{D}_{21 / 2}^{21}\end{array}$ & $\begin{array}{c}4 \\
6,7 b\end{array}$ \\
\hline $\begin{array}{l}3354.135 \\
3354.183 \\
3355.479 \\
3356.788 \\
3359.419\end{array}$ & $\begin{array}{l}1 h \\
1 h \\
10 \\
1 h \\
20 \mathrm{H}\end{array}$ & $\begin{array}{l}29805.41 \\
29804.98 \\
29793.47 \\
29781.86 \\
29758.53\end{array}$ & $\begin{array}{l}0.06 \\
0.13 \\
0.05 \\
0.02 \\
0.18\end{array}$ & $\begin{array}{l}a a^{4} \mathrm{~F}_{21 / 2}-v{ }^{4} \mathrm{H}_{31 / 2}^{\circ} \\
a{ }^{4} \mathrm{H}_{51 / 2}-x^{2} \mathrm{H}_{41 / 2}^{\circ} \\
b^{4} \mathrm{P}_{01 / 2}-u{ }^{4} \mathrm{D}_{01 / 2}^{\circ} \\
a^{4} \mathrm{~F}_{31 / 2}-w^{2} \mathrm{~F}_{21 / 2}^{\circ} \\
b^{4} \mathrm{P}_{21 / 2}-63583_{21 / 2}^{\circ}, \quad 11 / 2\end{array}$ & 6 \\
\hline $\begin{array}{ll}3360 . & 681 \\
3361 . & 054 \\
3362 . & 535 \\
3364.188 \\
3365 . & 137\end{array}$ & $\begin{array}{l}10 \\
2 \\
1 \\
6 \\
8 c w\end{array}$ & $\begin{array}{l}29747.36 \\
29744.06 \\
29730.96 \\
29716.35 \\
29707.97\end{array}$ & $\begin{array}{r}-0.08 \\
-0.08 \\
0.15 \\
0.08 \\
-0.04\end{array}$ & $\begin{array}{l}b^{4} \mathrm{D}_{31 / 2}-z^{2} \mathrm{D}_{21 / 2}^{\circ} \\
a^{2} \mathrm{H}_{51 / 2}-t^{4} \mathrm{G}_{51 / 2}^{\circ} \\
a^{6} \mathrm{D}_{11 / 2}-y^{4} \mathrm{P}_{01 / 2}^{\circ} \\
b^{4} \mathrm{D}_{11 / 2}-w^{4} \mathrm{D}_{01 / 2}^{\circ} \\
a^{4} \mathrm{H}_{41 / 2}-x^{2} \mathrm{H}_{51 / 2}^{0} ?\end{array}$ & 5 \\
\hline $\begin{array}{ll}\text { 3365. } & 693 \\
3366 . & 235 \\
3368 . & 086 \\
3368.194 \\
3369.188\end{array}$ & $\begin{array}{c}3 \\
10 \\
1 \\
7 h \\
10 h\end{array}$ & $\begin{array}{l}\text { 29703. } 06 \\
29698.28 \\
29681.96 \\
29681.00 \\
29672.25\end{array}$ & $\begin{array}{r}0.07 \\
-0.05 \\
-0.08 \\
0.02 \\
0.00\end{array}$ & 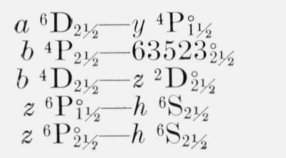 & 6 \\
\hline $\begin{array}{l}3370.813 \\
3372.087 \\
3375.223 \\
3376.527 \\
3378.871\end{array}$ & $\begin{array}{c}15 h \\
15 \\
3 \\
6 \\
3\end{array}$ & $\begin{array}{l}29657.95 \\
29646.74 \\
29619.20 \\
29607.76 \\
29587.22\end{array}$ & $\begin{array}{r}-0.10 \\
0.00 \\
0.07 \\
-0.07 \\
0.02\end{array}$ & $\begin{array}{c}z^{6} \mathrm{P}_{31 / 2}-h^{6} \mathrm{~S}_{21 / 2} \\
a^{4} \mathrm{~F}_{41 / 2}-x^{2} \mathrm{G}_{41 / 2}^{\circ} \\
a^{6} \mathrm{D}_{31 / 2}-y^{4} \mathrm{P}_{21 / 2}^{\circ} \\
a^{4} \mathrm{~F}_{31 / 2}-x^{2} \mathrm{G}_{31 / 2}^{0} \\
a^{4} \mathrm{D}_{31 / 2}-x \\
{ }^{6} \mathrm{D}_{21 / 2}^{0}\end{array}$ & 6 \\
\hline $\begin{array}{l}\text { 3380. } 283 \\
3380.444 \\
3380.817 \\
3381.981 \\
3382.673\end{array}$ & $\begin{array}{c}5 c \\
10 c \\
10 \\
4 c \\
3\end{array}$ & $\begin{array}{l}29574.86 \\
29573.46 \\
29570.19 \\
29560.01 \\
29553.96\end{array}$ & $\begin{array}{r}-0.03 \\
0.03 \\
0.03 \\
0.00 \\
-0.03\end{array}$ & $\begin{array}{l}a^{4} \mathrm{D}_{21 / 2}-y{ }^{4} \mathrm{D}_{31 / 2}^{\circ} \\
a^{4} \mathrm{D}_{31 / 2}-x{ }^{6} \mathrm{D}_{31 / 2}^{0} \\
b^{4} \mathrm{D}_{21 / 2}-w^{4} \mathrm{D}_{11 / 2}^{\circ} \\
a^{4} \mathrm{D}_{21 / 2}-y{ }^{4} \mathrm{D}_{21 / 2}^{\circ} \\
a^{4} \mathrm{D}_{21 / 2}-y{ }^{4} \mathrm{D}_{11 / 2}^{\circ}\end{array}$ & \\
\hline $\begin{array}{l}\text { 3383. } 585 \\
3386.863 \\
3389.287 \\
3393.843 \\
3396.367\end{array}$ & $\begin{array}{l}9 h \\
1 \\
2 \\
1 \\
2 h\end{array}$ & $\begin{array}{l}29546.00 \\
29517.40 \\
29496.29 \\
29456.70 \\
29434.81\end{array}$ & $\begin{array}{r}-0.07 \\
0.04 \\
0.09 \\
0.04 \\
0.05\end{array}$ & $\begin{array}{l}b^{4} \mathrm{P}_{21 / 2}-63371_{21 / 2}^{\circ} \\
a^{6} \mathrm{D}_{01 / 2}-y^{4} \mathrm{P}_{11 / 2}^{11 / 2} \\
a^{2} \mathrm{H}_{51 / 2}-w^{2} \mathrm{H}_{51 / 2}^{\circ} \\
a^{2} \mathrm{H}_{41 / 2}-w^{2} \mathrm{H}_{41 / 2}^{\circ} \\
b^{4} \mathrm{G}_{51 / 2}-u^{4} \mathrm{~F}_{41 / 2}^{0}\end{array}$ & $\begin{array}{l}6,7 b \\
6,7 b\end{array}$ \\
\hline $\begin{array}{l}\text { 3399. } 878 \\
3401.578 \\
3402.490\end{array}$ & $\begin{array}{l}1 \\
3 \\
2\end{array}$ & $\begin{array}{l}\text { 29404. } 41 \\
29389.72 \\
29381.84\end{array}$ & $\left\{\begin{array}{r}0.08 \\
0.03 \\
0.04 \\
-0.11\end{array}\right.$ & $\begin{array}{c}a{ }^{2} \mathrm{I}_{61 / 2}-u{ }^{4} \mathrm{H}_{61 / 2}^{\circ} \\
a^{4} \mathrm{D}_{11 / 2}-y{ }^{4} \mathrm{D}_{21 / 2}^{\circ} \\
a^{4} \mathrm{D}_{11 / 2}-y \\
b^{4} \mathrm{D}_{01 / 2}^{\circ} \mathrm{P}_{11 / 2}-x^{2} \mathrm{D}_{11 / 2}^{\circ}\end{array}$ & \\
\hline $\begin{array}{l}3406.122 \\
3407.962\end{array}$ & $\begin{array}{r}1 \\
20\end{array}$ & $\begin{array}{l}29350.51 \\
29334.66\end{array}$ & $\begin{array}{l}-0.17 \\
-0.01\end{array}$ & $\begin{array}{l}b^{4} \mathrm{G}_{41 / 2}-66981_{31 / 2}^{\circ 1} \\
a^{4} \mathrm{D}_{21 / 2}-x^{6} \mathrm{D}_{21 / 2}^{\circ}, 11 / 2\end{array}$ & 4 \\
\hline $\begin{array}{l}3408.188 \\
3409.561 \\
3410.345 \\
3410.800 \\
3411.816\end{array}$ & $\begin{array}{l}1 \\
3 c \\
1 \\
5 \\
1\end{array}$ & $\begin{array}{l}29332.72 \\
29320.91 \\
29314.17 \\
29310.26 \\
29301.53\end{array}$ & $\begin{array}{r}0.07 \\
0.01 \\
-0.04 \\
0.01 \\
0.00\end{array}$ & 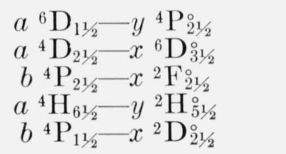 & \\
\hline
\end{tabular}


TABLE 1. Mn I-Classified lines-Continued

\begin{tabular}{|c|c|c|c|c|c|}
\hline 1 & 2 & 3 & 4 & 5 & 6 \\
\hline$\lambda$ & Int. & $\sigma$ & $\mathrm{o}-\mathrm{c}$ & Term designation & $\begin{array}{c}\text { Zeeman } \\
\text { type }\end{array}$ \\
\hline $\begin{array}{c}\text { A } \\
\text { Air } \\
3412.543 \\
3413.815 \\
3417.158 \\
3418.276 \\
3420.794\end{array}$ & $\begin{array}{l}3 h l \\
1 \\
6 \\
15 \\
50\end{array}$ & $\begin{array}{c}K \\
\text { 29295. } 29 \\
29284.37 \\
29255.73 \\
29246.15 \\
29224.63\end{array}$ & $\begin{array}{r}K \\
0.02 \\
0.05 \\
-0.06 \\
0.01 \\
-0.03\end{array}$ & $\begin{array}{l}a^{2} \mathrm{~F}_{31 / 2}-t^{4} \mathrm{G}_{21 / 2}^{\circ} \\
a^{4} \mathrm{D}_{012}-y^{4} \mathrm{D}_{11 / 2}^{\circ} \\
b{ }^{4} \mathrm{P}_{21 / 2}-y^{2} \mathrm{D}_{21 / 2}^{2} \\
b^{4} \mathrm{D}_{31 / 2}-w^{4} \mathrm{D}_{21 / 2}^{\circ} \\
a^{4} \mathrm{H}_{61 / 2}-w^{4} \mathrm{H}_{61 / 2}^{\circ}\end{array}$ & 6, $\stackrel{5}{7 b}$ \\
\hline $\begin{array}{l}\text { 3421. } 491 \\
3422.418 \\
3422.826 \\
3423.137 \\
3423.841\end{array}$ & $\begin{array}{r}1 \\
1 \\
10 \\
2 \\
10\end{array}$ & $\begin{array}{l}\text { 29218. } 67 \\
29210.76 \\
29207.28 \\
29204.63 \\
29198.62\end{array}$ & $\begin{array}{r}0.06 \\
0.08 \\
-0.05 \\
0.04 \\
0.01\end{array}$ & $\begin{array}{l}b^{4} \mathrm{G}_{21 / 2}-67008_{21 / 2}^{\circ} \\
b^{4} \mathrm{G}_{51 / 2}^{\circ}-v^{2} \mathrm{G}_{41 / 2}^{\circ} \\
a^{4} \mathrm{H}_{51 / 2}-w^{4} \mathrm{H}_{51 / 2}^{\circ} \\
a^{4} \mathrm{H}^{4} z^{1 / 2}-z^{2} \mathrm{H}_{11 / 2}^{\circ} \\
a^{4} \mathrm{H}_{51 / 2}-y^{2} \mathrm{H}_{51 / 2}^{\circ}\end{array}$ & 6, $7 b$ \\
\hline $\begin{array}{l}3424.375 \\
3425.929 \\
3427.865 \\
3427.957 \\
3428.782\end{array}$ & $\begin{array}{r}5 \\
1 \\
10 \\
3 \\
15\end{array}$ & $\begin{array}{l}29194.07 \\
29180.82 \\
29164.34 \\
29163.56 \\
29156.55\end{array}$ & $\begin{array}{r}-0.02 \\
0.08 \\
-0.01 \\
-0.02 \\
0.00\end{array}$ & $\begin{array}{l}a{ }^{4} \mathrm{H}_{51 / 2}-w^{4} \mathrm{H}_{41 / 2}^{\circ} \\
b^{4} \mathrm{D}_{21 / 2}-w^{4} \mathrm{D}_{21 / 2}^{\circ} \\
a^{4} \mathrm{D}_{11 / 2}-x{ }^{6} \mathrm{D}_{21 / 2,11 / 2}^{\circ} \\
a^{4} \mathrm{D}_{11 / 2}-x{ }^{6} \mathrm{D}_{01 / 2}^{\circ} \\
b^{4} \mathrm{D}_{01 / 2}-u{ }^{4} \mathrm{P}_{01 / 2}^{\circ}\end{array}$ & 6 \\
\hline $\begin{array}{l}3429.155 \\
3429.741 \\
3430.428 \\
3433.025 \\
3433.572\end{array}$ & $\begin{array}{c}4 \\
2 \\
1 \\
5 h \\
50\end{array}$ & $\begin{array}{l}29153.37 \\
29148.39 \\
29142.56 \\
29120.51 \\
29115.87\end{array}$ & $\begin{array}{r}0.01 \\
0.05 \\
-0.02 \\
0.04 \\
-0.06\end{array}$ & $\begin{array}{l}b^{4} \mathrm{G}_{51 / 2}-u{ }^{4} \mathrm{G}_{51 / 2}^{\circ} \\
b^{4} \mathrm{G}_{51 / 2}-u{ }^{4} \mathrm{H}_{61 / 2}^{\circ} \\
b^{4} \mathrm{D}_{11 / 2}-u{ }^{4} \mathrm{P}_{01 / 2}^{\circ} \\
b^{4} \mathrm{P}_{11 / 2}-63583_{21 / 2,1 / 2}^{\circ}-6358 \\
b^{4} \mathrm{D}_{31 / 2}-v{ }^{4} \mathrm{D}_{31 / 2}^{\circ}\end{array}$ & 6 \\
\hline $\begin{array}{l}\text { 3433. } 911 \\
\text { 3434. } 473 \\
\text { 3434. } 823 \\
\text { 3435. } 193 \\
\text { 3435. } 254\end{array}$ & $\begin{array}{l}1 \\
2 \\
1 \\
5 \\
4\end{array}$ & $\begin{array}{l}\text { 29113. } 00 \\
29108.23 \\
29105.27 \\
29102.13 \\
29101.62\end{array}$ & $\begin{array}{r}-0.02 \\
-0.05 \\
0.04 \\
-0.05 \\
-0.03\end{array}$ & $\begin{array}{l}a^{4} \mathrm{H}_{51 / 2}-w^{4} \mathrm{H}_{61 / 2}^{\circ}{ }^{\circ} \\
b^{4} \mathrm{D}_{21 / 2}-v \\
{ }^{4} \mathrm{D}_{11 / 2}^{\circ} \\
a^{4} \mathrm{H}_{41 / 2}-y \\
b^{2} \mathrm{H}_{51 / 2}^{\circ} \mathrm{D}_{11 / 2}-v \\
{ }^{4} \mathrm{D}_{11 / 2}^{\circ} \\
b^{4} \mathrm{D}_{11 / 2}-v{ }^{4} \mathrm{D}_{01 / 2}^{\circ}\end{array}$ & $\begin{array}{l}\mathrm{P}-\mathrm{B} \\
\mathrm{P}-\mathrm{B}\end{array}$ \\
\hline 3435. 375 & 5 & 29100.59 & $\left\{\begin{array}{r}-0.12 \\
0.04\end{array}\right.$ & $\begin{array}{l}a{ }^{4} \mathrm{H}_{41 / 2}-w^{4} \mathrm{H}_{41 / 2}^{\circ} \\
a^{4} \mathrm{H}_{31 / 2}-63523^{2}{ }^{2} / 1\end{array}$ & $6,7 b$ \\
\hline $\begin{array}{l}3439.579 \\
3439.673 \\
3440.044 \\
3440.769\end{array}$ & $\begin{array}{r}3 \\
3 \\
10 \\
1\end{array}$ & $\begin{array}{l}\text { 29065. } 02 \\
29064.23 \\
29061.10 \\
29054.97\end{array}$ & $\begin{array}{r}0.02 \\
0.00 \\
-0.09 \\
-0.12\end{array}$ & $\begin{array}{l}a^{4} \mathrm{D}_{01 / 2}-x^{6} \mathrm{D}_{11 / 2}^{\circ 1 / 2} \\
a^{4} \mathrm{D}_{01 / 2}-x{ }^{6} \mathrm{D}_{01 / 2}^{0} \\
b{ }^{4} \mathrm{D}_{21 / 2}-v{ }^{4} \mathrm{D}_{21 / 2}^{\circ} \\
b^{4} \mathrm{D}_{11 / 2}-v{ }^{4} \mathrm{D}_{21 / 2}^{\circ}\end{array}$ & 6 \\
\hline $\begin{array}{l}\text { 3440. } 907 \\
\text { 3441. } 177 \\
3442.756 \\
3443.657 \\
3444.237\end{array}$ & $\begin{array}{l}1 \\
2 \\
5 \\
5 h \\
2 h c\end{array}$ & $\begin{array}{l}\text { 29053. } 79 \\
29051.53 \\
29038.20 \\
29030.60 \\
29025.72\end{array}$ & $\begin{array}{l}-0.07 \\
-0.02 \\
-0.06 \\
-0.03 \\
-0.26\end{array}$ & $\begin{array}{c}b{ }^{4} \mathrm{G}_{21 / 2}-u^{4} \mathrm{~F}_{11 / 2}^{\circ} \\
a{ }^{4} \mathrm{H}_{41 / 2}-w^{4} \mathrm{H}_{31 / 2}^{8} \\
a{ }^{4} \mathrm{H}_{51 / 2}-z^{2} \mathrm{H}_{51 / 2}^{\circ} \\
a^{4} \mathrm{H}_{41 / 2}-6337^{1} 4_{41 / 3}^{8} \\
b^{4} \mathrm{P}_{21 / 2}-w^{6} \mathbf{D}_{31 / 2}^{\circ}\end{array}$ & $6,7 b$ \\
\hline $\begin{array}{l}3446.519 \\
3446.766 \\
3446.822 \\
3448.429 \\
3449.036\end{array}$ & $\begin{array}{l}1 \\
2 \\
5 \\
1 h \\
1\end{array}$ & $\begin{array}{l}\text { 29006. } 50 \\
29004.42 \\
29003.95 \\
28990.44 \\
28985.34\end{array}$ & $\begin{array}{r}-0.01 \\
0.01 \\
-0.06 \\
0.00 \\
0.00\end{array}$ & $\begin{array}{l}b{ }^{4} \mathrm{D}_{31 / 2}-x^{4} \mathrm{~F}_{21 / 2}^{\circ} \\
b{ }^{4} \mathrm{D}_{01 / 2}-x{ }^{4} \mathrm{~F}_{11 / 2}^{\circ} \\
a{ }^{4} \mathrm{H}_{41 / 2}-y^{2} \mathrm{H}_{41 / 2}^{\circ} \\
b{ }^{4} \mathrm{D}_{11 / 2}-x^{4} \mathrm{~F}_{11 / 2}^{\circ} \\
b^{4} \mathrm{D}_{31 / 2}-w^{4} \mathrm{D}_{31 / 2}^{0}\end{array}$ & 6 \\
\hline $\begin{array}{l}3450.607 \\
3450.932 \\
3451.475 \\
3452.201 \\
3452.440\end{array}$ & $\begin{array}{l}20 \\
1 h \\
7 \\
5 \\
5 w\end{array}$ & $\begin{array}{l}28972.14 \\
28969.41 \\
28964.85 \\
28958.76 \\
28956.75\end{array}$ & $\begin{array}{r}-0.04 \\
-0.14 \\
0.01 \\
0.02 \\
0.18\end{array}$ & $\begin{array}{l}a{ }^{4} \mathrm{H}_{31 / 2}-w^{4} \mathrm{H}_{31 / 2}^{\circ} \\
b{ }^{4} \mathrm{G}_{41 / 2}-66600^{\circ}{ }^{\circ} 1 / 2 \\
b{ }^{4} \mathrm{D}_{21 / 2}-u{ }^{4} \mathrm{P}_{11 / 2}^{11} \\
b{ }^{4} \mathrm{D}_{11 / 2}-u{ }^{4} \mathrm{P}_{11 / 2}^{1} \\
a^{4} \mathrm{D}_{31 / 2}-u{ }^{6} \mathrm{P}_{31 / 2}^{\circ}\end{array}$ & 6 \\
\hline $\begin{array}{l}3453.426 \\
3453.505 \\
3453.861 \\
3454.110 \\
3454.309\end{array}$ & $\begin{array}{l}2 h \\
2 h w \\
1 \\
2 \\
1\end{array}$ & $\begin{array}{l}\text { 28948. } 49 \\
28947.83 \\
\text { 28944. } 84 \\
\text { 28942. } 76 \\
\text { 28941. } 09\end{array}$ & $\begin{array}{r}0.01 \\
-0.06 \\
\left\{\begin{array}{r}-0.04 \\
-0.04 \\
-0.22 \\
-0.02\end{array}\right.\end{array}$ & $\begin{array}{l}a{ }^{6} \mathrm{D}_{41 / 2}-y^{8} \mathrm{P}_{31 / 2}^{\circ} \\
b^{4} \mathrm{G}_{21 / 2}-v^{2} \mathrm{G}_{31 / 2}^{\circ} \\
a{ }^{4} \mathrm{H}_{41 / 2}-z^{2} \mathrm{H}_{51 / 2}^{\circ} \\
a^{4} \mathrm{H}_{41 / 2}-x^{2} \mathrm{~F}_{31 / 2}^{5} \\
b^{4} \mathrm{G}_{41 / 2}-u^{4} \mathrm{G}_{51 / 2}^{\circ} \\
b^{4} \mathrm{D}_{21 / 2}-x^{4} \mathrm{~F}_{21 / 2}^{\circ}\end{array}$ & \\
\hline
\end{tabular}


Table 1. Mn I-Classified lines-Continued

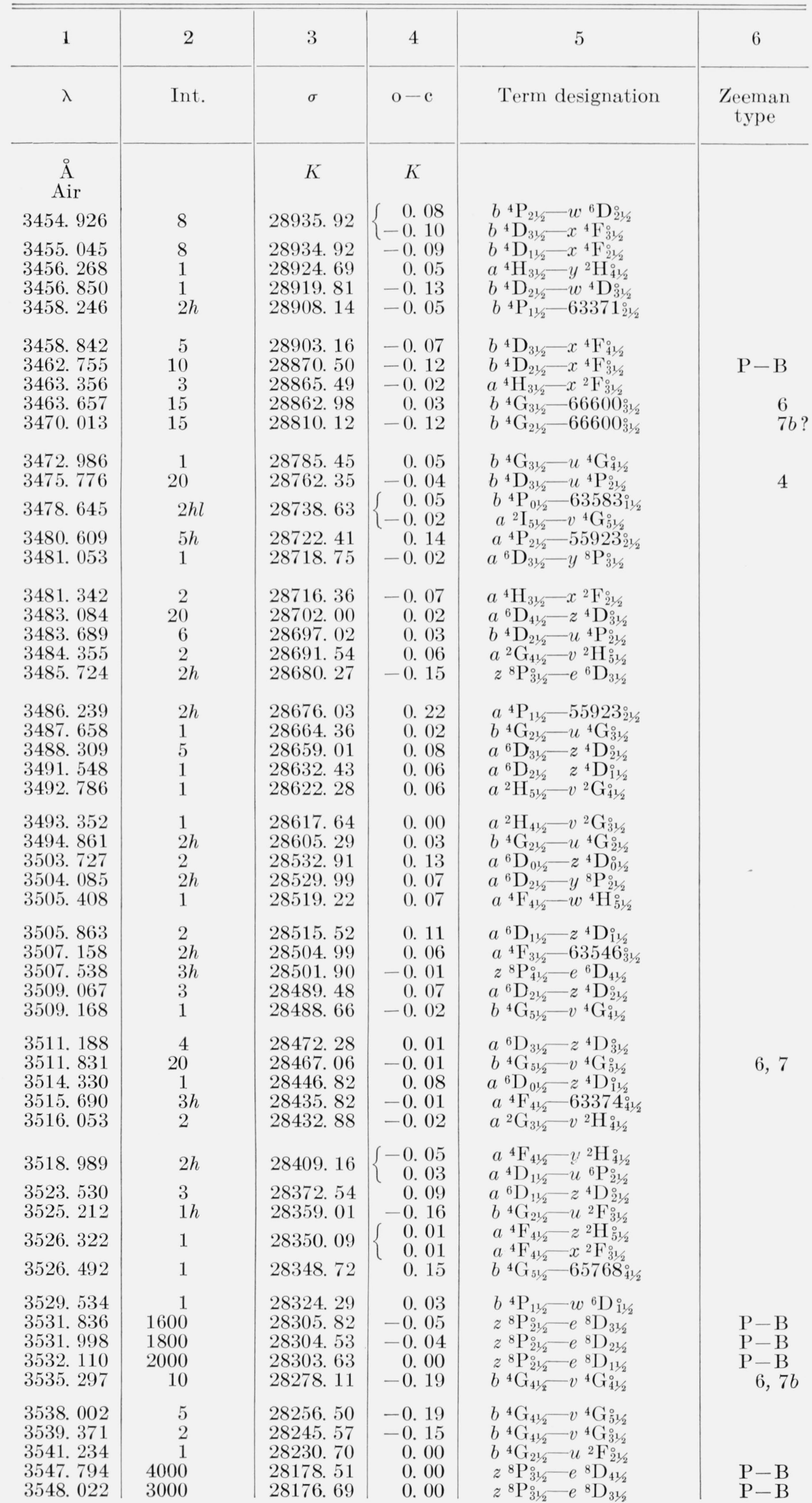


Table 1. Mn I-Classified lines-Continued

\begin{tabular}{|c|c|c|c|c|c|}
\hline 1 & 2 & 3 & 4 & 5 & 6 \\
\hline$\lambda$ & Int. & $\sigma$ & $\mathrm{o}-\mathrm{c}$ & Term designation & $\begin{array}{c}\text { Zeeman } \\
\text { type }\end{array}$ \\
\hline $\begin{array}{c}\text { A } \\
\text { Air } \\
3548.182 \\
3552.757 \\
3552.901 \\
3553.121 \\
3555.066\end{array}$ & $\begin{array}{r}1000 \\
10 \\
4 \\
1 \\
2\end{array}$ & $\begin{array}{c}K \\
28175.43 \\
28139.14 \\
28138.00 \\
28136.26 \\
28120.87\end{array}$ & $\begin{array}{c}K \\
0.04 \\
0.02 \\
-0.19 \\
0.08 \\
0.21\end{array}$ & 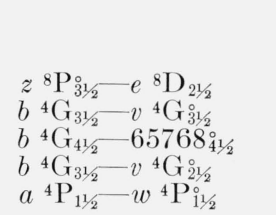 & $\mathrm{P}-\mathrm{B}$ \\
\hline $\begin{array}{l}3559.373 \\
3559.434 \\
3559.808 \\
3561.736 \\
3566.330\end{array}$ & $\begin{array}{r}2 \\
2 \\
10 \\
1 \\
1\end{array}$ & $\begin{array}{l}\text { 28086. } 84 \\
28086.36 \\
28083.41 \\
28068.21 \\
28032.05\end{array}$ & $\begin{array}{r}0.03 \\
-0.05 \\
-0.06 \\
-0.01 \\
0.14\end{array}$ & 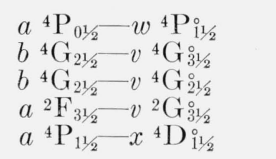 & \\
\hline $\begin{array}{l}3566.731 \\
3569.494 \\
3569.804 \\
3570.028 \\
3572.354\end{array}$ & $\begin{array}{r}3 \\
6000 \\
3000 \\
1000 \\
1\end{array}$ & $\begin{array}{l}\text { 28028. } 91 \\
28007.21 \\
\text { 28004. } 78 \\
28003.02 \\
27984.79\end{array}$ & $\begin{array}{r}-0.01 \\
0.00 \\
0.00 \\
0.06 \\
0.16\end{array}$ & $\begin{array}{l}a^{2} \mathrm{H}_{41 / 2}-u^{2} \mathrm{~F}_{31 / 2}^{\circ} \\
z^{8} \mathrm{P}_{41 / 2}^{\circ}-e^{8} \mathrm{D}_{51 / 2}^{\circ} \\
z^{8} \mathrm{P}_{41 / 2}^{\circ}-e^{8} \mathrm{D}_{41 / 2}^{\circ} \\
z^{8} \mathrm{P}_{41 / 2}^{\circ}-e^{8} \mathrm{D}_{31 / 2} \\
a^{4} \mathrm{P}_{21 / 2}-x^{4} \mathrm{D}_{21 / 2}^{\circ}\end{array}$ & $\begin{array}{l}\mathrm{P}-\mathrm{B} \\
\mathrm{P}-\mathrm{B} \\
\mathrm{P}-\mathrm{B}\end{array}$ \\
\hline $\begin{array}{l}3575.356 \\
3575.967 \\
3576.073 \\
3576.303 \\
3577.187\end{array}$ & $\begin{array}{l}1 \\
4 \mathrm{H} \\
8 \mathrm{H} \\
4 \mathrm{H} \\
2 \mathrm{H}\end{array}$ & $\begin{array}{l}27961.29 \\
27956.51 \\
27955.68 \\
27953.89 \\
27946.98\end{array}$ & $\begin{array}{r}-0.03 \\
0.00 \\
-0.01 \\
-0.01 \\
0.02\end{array}$ & 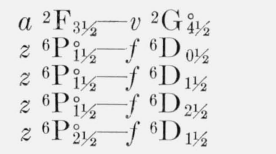 & \\
\hline $\begin{array}{l}3577.416 \\
3577.870 \\
3578.285 \\
3579.637 \\
3580.112\end{array}$ & $\begin{array}{c}5 \mathrm{H} \\
2000 \\
3 \\
8 \\
40 \mathrm{H} l\end{array}$ & $\begin{array}{l}27945.19 \\
27941.64 \\
27938.40 \\
27927.85 \\
27924.14\end{array}$ & $\begin{array}{r}0.02 \\
0.01 \\
0.23 \\
-0.31 \\
0.00\end{array}$ & 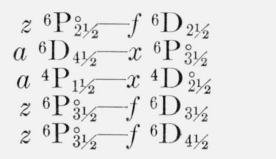 & 4 \\
\hline $\begin{array}{l}3581.545 \\
3582.425 \\
3583.187 \\
3583.675 \\
3585.966\end{array}$ & $\begin{array}{l}5 \mathrm{H} w \\
20 c \\
2 \\
40 \\
1\end{array}$ & $\begin{array}{l}27912.97 \\
27906.11 \\
27900.18 \\
27896.38 \\
27878.56\end{array}$ & $\begin{array}{r}0.20 \\
0.13 \\
-0.04 \\
-0.07 \\
-0.05\end{array}$ & $\begin{array}{l}a^{4} \mathrm{H}_{41 / 2}-w^{6} \mathrm{D}_{31 / 2}^{0} \\
a^{4} \mathrm{P}_{21 / 2}-x^{4} \mathrm{D}_{31 / 2}^{3} \\
a^{2} \mathrm{H}_{51 / 2}-v{ }^{4} \mathrm{G}_{41 / 2}^{0} \\
a^{4} \mathrm{D}_{21 / 2}-x^{4} \mathrm{P}_{11 / 2}^{0} \\
a^{2} \mathrm{H}_{51 / 2}-v^{4} \mathrm{G}_{51 / 2}^{1}\end{array}$ & 4 \\
\hline $\begin{array}{l}3586.540 \\
3590.664 \\
3591.806 \\
3592.556 \\
3595.110\end{array}$ & $\begin{array}{c}1000 h \\
2 \\
15 \\
1 \\
500 h\end{array}$ & $\begin{array}{l}27874.10 \\
27842.09 \\
27833.23 \\
27827.42 \\
27807.65\end{array}$ & $\begin{array}{r}-0.01 \\
0.05 \\
-0.03 \\
-0.02 \\
0.00\end{array}$ & 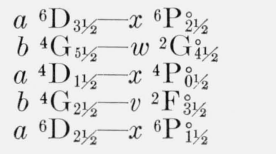 & 4 \\
\hline $\begin{array}{l}3597.560 \\
3599.504 \\
3601.268 \\
3601.772 \\
3604.670\end{array}$ & $\begin{array}{l}1 \\
4 \mathrm{H} w \\
20 \\
20 c \\
15 c\end{array}$ & $\begin{array}{l}27788.72 \\
27773.71 \\
27760.11 \\
27756.22 \\
27733.91\end{array}$ & $\begin{array}{r}-0.02 \\
0.02 \\
0.00 \\
0.01 \\
0.00\end{array}$ & $\begin{array}{l}a{ }^{2} \mathrm{H}_{41 / 2}-v^{4} \mathrm{G}_{41 / 2}^{\circ} \\
a^{4} \mathrm{H}_{61 / 2}-y^{2} \mathrm{I}_{61 / 2}^{\circ} \\
a^{2} \mathrm{H}_{51 / 2}-65768^{\circ 1 / 2} \\
a^{4} \mathrm{D}_{21 / 2}-x^{4} \mathrm{P}_{21 / 2}^{\circ} \\
a^{4} \mathrm{D}_{01 / 2}-x^{4} \mathrm{P}_{01 / 2}^{\circ}\end{array}$ & $\begin{array}{l}4 \\
6 \\
6\end{array}$ \\
\hline $\begin{array}{l}3605.683 \\
3606.489 \\
3606.702 \\
3607.530 \\
3608.485\end{array}$ & $\begin{array}{l}20 \\
2 \mathrm{H} \\
1 \mathrm{H} \\
1000 \\
1000\end{array}$ & $\begin{array}{l}27726.12 \\
27719.92 \\
27718.28 \\
27711.92 \\
27704.59\end{array}$ & $\begin{array}{r}-0.01 \\
-0.04 \\
0.00 \\
0.00 \\
0.00\end{array}$ & $\begin{array}{c}a{ }^{4} \mathrm{D}_{11 / 2}-x{ }^{4} \mathrm{P}_{11 / 2}^{\circ} \\
a^{4} \mathrm{~F}_{31 / 2}-w^{6} \mathrm{D}^{6}{ }^{21 / 2} \\
a^{4} \mathrm{D}_{31 / 2}-x{ }^{6} \mathrm{~F}_{31 / 2}^{\circ} \\
a^{6} \mathrm{D}_{31 / 2}-x{ }^{6} \mathrm{P}_{31 / 2}^{0} \\
a^{6} \mathrm{D}_{21 / 2}-x{ }^{6} \mathrm{P}_{21 / 2}^{01 / 2}\end{array}$ & $\begin{array}{l}6 \\
6\end{array}$ \\
\hline $\begin{array}{l}3610.298 \\
3612.612 \\
3615.375 \\
3618.646 \\
3619.272\end{array}$ & $\begin{array}{c}1000 \\
2 \mathrm{H} \\
10 \mathrm{H} \\
2 \\
600\end{array}$ & $\begin{array}{l}27690.68 \\
27672.94 \\
27651.78 \\
27626.80 \\
27622.02\end{array}$ & $\begin{array}{r}-0.01 \\
0.29 \\
0.03 \\
0.02 \\
0.00\end{array}$ & 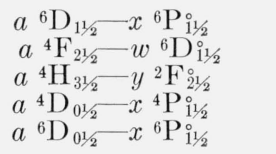 & 5 \\
\hline $\begin{array}{l}3620.751 \\
3621.483 \\
3623.783 \\
3626.299 \\
3629.738\end{array}$ & $\begin{array}{c}1 h \\
6 \mathrm{H} w \\
500 h \\
3 \mathrm{H} w \\
400 h\end{array}$ & $\begin{array}{l}27610.73 \\
27605.15 \\
27587.63 \\
27568.49 \\
27542.38\end{array}$ & $\begin{array}{r}-0.04 \\
-0.01 \\
0.00 \\
-0.06 \\
-0.02\end{array}$ & $\begin{array}{l}a{ }^{4} \mathrm{H}_{31 / 2}-y^{2} \mathrm{~F}_{31 / 2}^{\circ} \\
a{ }^{4} \mathrm{H}_{61 / 2}-61744_{51 / 2}^{\circ} \\
a{ }^{6} \mathrm{D}_{11 / 2}-x^{6} \mathrm{P}^{\circ}{ }^{21 / 2} \\
a^{4} \mathrm{H}_{51 / 2}-y^{2} \mathrm{I}_{51 / 2}^{\circ} \\
a^{6} \mathrm{D}_{21 / 2}-x^{6} \mathrm{P}_{31 / 2}^{\circ}\end{array}$ & 5 \\
\hline
\end{tabular}


Table 1. Mn I-Classified lines-Continued

\begin{tabular}{|c|c|c|c|c|c|}
\hline 1 & 2 & 3 & 4 & 5 & 6 \\
\hline$\lambda$ & Int. & $\sigma$ & $o-c$ & Term designation & $\begin{array}{l}\text { Zeeman } \\
\text { type }\end{array}$ \\
\hline$\stackrel{\AA}{\mathrm{A}}$ & & $K$ & $K$ & & \\
\hline 3635. 699 & 10 & 27497. 22 & 0.03 & $a^{2} \mathrm{H}_{41 / 2}-v^{2} \mathrm{~F}_{31 / 2}^{\circ}$ & 4 \\
\hline 3636. 190 & $2 \mathrm{H} w$ & 27493.51 & -0.01 & $a^{4} \mathrm{H}_{51 / 2}^{51 / 2} \quad 61744_{51 / 2}^{\circ}$ & \\
\hline 3638. 032 & $1 h$ & 27479.59 & 0. 09 & $a^{2} \mathrm{~F}_{31 / 2}-u^{2} \mathrm{~F}_{31 / 2}^{0}$ & \\
\hline $\begin{array}{l}3639.145 \\
3639.580\end{array}$ & $\begin{array}{l}5 \\
1\end{array}$ & $\begin{array}{l}27471.18 \\
27467.90\end{array}$ & $\begin{array}{l}0.02 \\
0.14\end{array}$ & $\begin{array}{l}a{ }^{6} \mathrm{D}_{41 / 2}-z^{4} \mathrm{~F}^{31 / 2} \\
b^{4} \mathrm{G}_{51}-v^{4} \mathrm{H}^{81}\end{array}$ & \\
\hline 3640. 086 & $15 h$ & 27464.08 & $\left\{\begin{array}{l}0.16 \\
0.08\end{array}\right.$ & $\begin{array}{l}a{ }^{4} \mathrm{~F}_{31 / 2}-v^{4} \mathrm{~F}_{31 / 2}^{0} \\
a^{4} \mathrm{H}_{512}-v^{2} \mathrm{G}^{0}\end{array}$ & \\
\hline 3641. 405 & $30 h$ & 27454.13 & 0.01 & $a^{4} \mathrm{~F}_{41 / 2}-v^{4} \mathrm{~F}_{41 / 2}^{0}$ & $6,7 b$ \\
\hline 3643. 018 & 3 & 27441.98 & -0.06 & $a^{4} \mathrm{H}_{41 / 2}-y^{2} \mathrm{G}_{31 / 2}^{\circ}$ & 5 \\
\hline 3646. 707 & 3 & 27414. 22 & -0.07 & $a^{6} \mathrm{D}_{31 / 2}-z^{4} \mathrm{~F}_{21 / 2}^{0}$ & \\
\hline 3648. 698 & 6 & 27399. 26 & -0.03 & $b^{4} \mathrm{G}_{51 / 2}-v{ }^{4} \mathrm{H}_{51 / 2}^{0}$ & 6 \\
\hline 3652. 292 & $3 \mathrm{H}$ & 27372.30 & -0.08 & $a{ }^{4} \mathrm{~F}_{21 / 2}-v^{4} \mathrm{~F}_{21 / 2}^{\circ}$ & \\
\hline 3652. 510 & $1 h$ & 27370.66 & 0.04 & $a^{4} \mathrm{H}_{41 / 2}-y{ }^{2} \mathrm{G}_{41 / 2}^{\circ / 2}$ & \\
\hline 3653. 514 & 2 & 27363.14 & -0.07 & $a^{6} \mathrm{D}_{21 / 2}-z^{4} \mathrm{~F}_{11 / 2}^{1 / 2}$ & \\
\hline $\begin{array}{l}3653.581 \\
3654.291\end{array}$ & $\begin{array}{l}2 \\
2 h\end{array}$ & $\begin{array}{l}27362.64 \\
27357.32\end{array}$ & $\begin{array}{l}-0.03 \\
-0.28\end{array}$ & $\begin{array}{l}a^{4} \mathrm{H}_{31 / 2}-y^{2} \mathrm{G}_{31 / 2}^{\circ} \\
b{ }^{4} \mathrm{G}_{41 / 2}-w^{2} \mathrm{~F}_{31 / 2}^{\circ}\end{array}$ & \\
\hline 3655. 097 & $3 h$ & 27351. 29 & $\left\{\begin{array}{l}-0.16 \\
0.96\end{array}\right.$ & $a^{4} \mathrm{~F}_{31 / 2}-v{ }^{4} \mathrm{~F}_{41 / 2}^{1}$ & \\
\hline 3657. 906 & $20 c$ & 27330. 29 & $\begin{array}{r}0.20 \\
-0.04\end{array}$ & $a^{4} \mathrm{H}_{61 / 2}-w^{4}{ }^{4} \mathrm{G}_{51 / 2}^{21 / 2}$ & 5 \\
\hline 3660.404 & 100 & 27311.64 & 0.00 & $b^{4} \mathrm{G}_{51 / 2}-v{ }^{4} \mathrm{H}_{61 / 2}^{0}$ & 4 \\
\hline 3663. 135 & $1 h$ & 27291. 27 & 0.02 & $a^{4} \mathrm{H}_{31 / 2}-y^{2} \mathrm{G}_{41 / 2}^{\circ}$ & \\
\hline 3663. 373 & $2 h$ & 27289.50 & -0.21 & $b^{4} \mathrm{G}_{41 / 2}^{1}-v^{4} \mathrm{H}_{31 / 2}^{10}$ & \\
\hline 2667. 714 & 10 & 27257. 21 & -0.17 & $b{ }^{4} \mathrm{G}_{41 / 2}-v^{4} \mathrm{H}_{41 / 2}^{\circ}$ & 6 \\
\hline 3668. 201 & 1 & 27253.59 & 0.01 & $a^{2} \mathrm{H}_{51 / 2}-w^{2} \mathrm{G}_{41 / 2}^{\circ / 2}$ & \\
\hline 3668.547 & $1 h$ & 27251. 02 & 0.02 & $b^{4} \mathrm{G}_{31 / 2}-w^{2} \mathrm{~F}_{31 / 2}$ & \\
\hline 3669. 198 & 4 & 27246.18 & -0.07 & $a^{6} \mathrm{D}_{11 / 2}^{0}-z^{4} \mathrm{~F}_{11 / 2}^{0}$ & \\
\hline 3669.398 & 15 & 27244.70 & -0.07 & $a{ }^{6} \mathrm{D}_{21 / 2}-z^{4} \mathrm{~F}_{21 / 2}$ & \\
\hline 3669. 837 & 50 & 27241. 44 & -0.01 & $a^{6} \mathrm{D}_{31 / 2}-z^{4} \mathrm{~F}_{31 / 2}^{\circ}$ & \\
\hline 3670.505 & 100 & 27236.48 & 0.01 & $a^{6} \mathrm{D}_{41 / 2}-z^{4} \mathrm{~F}_{41 / 2}^{0}$ & 6 \\
\hline 3672. 037 & 1 & 27225.12 & -0.03 & $a^{4} \mathrm{~F}_{11 / 2}-v^{4} \mathrm{~F}_{11 / 2}^{0}$ & \\
\hline 3672. 915 & 1 & 27218.61 & -0.08 & $a^{4} \mathrm{H}_{51 / 2}-w^{4} \mathrm{G}_{51 / 2}^{2}$ & \\
\hline 3673.515 & 2 & 27214. 16 & 0.00 & $a^{2} \mathrm{~F}_{21 / 2}-u^{2} \mathrm{~F}_{31 / 2}^{0}$ & \\
\hline 3674. 514 & 1 & 27206. 76 & 0.02 & $a^{2} \mathrm{~F}_{31 / 2}-v^{4} \mathrm{G}_{31 / 2}^{\circ}$ & \\
\hline 3675.670 & $10 \mathrm{Hl}$ & 27198. 21 & -0.08 & $b^{4} \mathrm{G}_{21 / 2}-w^{2} \mathrm{~F}_{31 / 2}^{\circ}$ & \\
\hline 3676.960 & 80 & 27188.67 & -0.24 & $b{ }^{4} \mathrm{G}_{41 / 2}-v{ }^{4} \mathrm{H}_{51 / 2}^{0}$ & 4 \\
\hline 3677. 469 & 1 & 27184. 90 & -0.05 & $a^{2} \mathrm{H}_{41 / 2}-w^{2} \mathrm{G}_{31 / 2}^{0}$ & \\
\hline 3677.716 & $4 h$ & 27183. 08 & -0.03 & $b{ }^{4} \mathrm{G}_{31 / 2}-v{ }^{4} \mathrm{H}_{31 / 2}^{0}$ & \\
\hline 3678. 470 & 3 & 27177.50 & -0.08 & $a^{6} \mathrm{D}_{01 / 2}-z^{4} \mathrm{~F}_{11 / 2}^{\circ}$ & \\
\hline 3680.146 & 20 & 27165. 12 & -0.08 & $b^{4} \mathrm{G}_{51 / 2}-x^{2} \mathrm{G}_{41 / 2}^{102}$ & 4 \\
\hline 3682. 090 & 60 & 27150.79 & 0.01 & $b^{4} \mathrm{G}_{31 / 2}-v{ }^{4} \mathrm{H}_{41 / 2}$ & 4 \\
\hline 2683. 363 & 4 & 27141.40 & -0.04 & $a^{4} \mathrm{H}_{41 / 2}-w^{4} \mathrm{G}_{41 / 2}$ & \\
\hline 3684.522 & 20 & 27132.87 & 0.00 & $b{ }^{4} \mathrm{D}_{31 / 2}-v{ }^{4} \mathrm{P}_{21 / 2}^{\circ}$ & 4 \\
\hline 3684. 866 & $20 h$ & 27130.33 & -0.07 & $b^{4} \mathrm{G}_{21 / 2}-v^{4} \mathrm{H}_{31 / 2}^{\circ}$ & \\
\hline 3685. 215 & 15 & 27127. 76 & -0.05 & $a^{6} \mathrm{D}_{11 / 2}-z{ }^{4} \mathrm{~F}_{21 / 2}^{0}$ & \\
\hline 3685.561 & 7 & 27125. 22 & -0.09 & $a{ }^{4} \mathrm{H}_{41 / 2}-w^{4} \mathrm{G}_{51 / 2}^{2}$ & \\
\hline 3689. 097 & 2 & 27099. 22 & $\left\{\begin{array}{l}0.01 \\
0.10\end{array}\right.$ & $a^{2} \mathrm{~F}_{31 / 2}-65768_{41 / 2}^{\circ}$ & 4 \\
\hline 3689. 989 & $1 h$ & 27092.67 & 0.12 & $b{ }^{4} \mathrm{D}_{21 / 2}-y{ }^{4} \mathrm{~S}_{11 / 2}^{01 / 2}$ & \\
\hline 3690. 808 & 10 & 27086.66 & -0.01 & $a^{4} \mathrm{H}_{61 / 2}-y{ }^{4} \mathrm{I}_{61 / 2}^{\circ}$ & \\
\hline 3690. 933 & $5 h$ & 27085. 74 & $\left\{\begin{array}{r}0.05 \\
-0.25\end{array}\right.$ & $a^{2}{ }^{2} \mathrm{~F}_{21 / 2}-u^{2} \mathrm{~F}_{21 / 2}^{0}$ & \\
\hline 3692. 187 & $2 h$ & 27076.54 & $\begin{array}{r}-0.20 \\
-0.02\end{array}$ & $a^{4} \mathrm{D}_{31 / 2}-y^{4} \mathrm{~F}^{4}{ }^{21 / 2}$ & \\
\hline 3692. 817 & 40 & 27071.92 & -0.01 & $a^{6} \mathrm{D}_{21 / 2}-z^{4} \mathrm{~F}_{31 / 2}^{272}$ & 4 \\
\hline 3693. 426 & 4 & 27067.46 & -0.01 & $b^{4} \mathrm{D}_{21 / 2}^{2 / 2}-v{ }^{4} \mathrm{P}_{21 / 2}^{0}$ & \\
\hline
\end{tabular}


TABle 1. Mn I-Classified lines-Continued

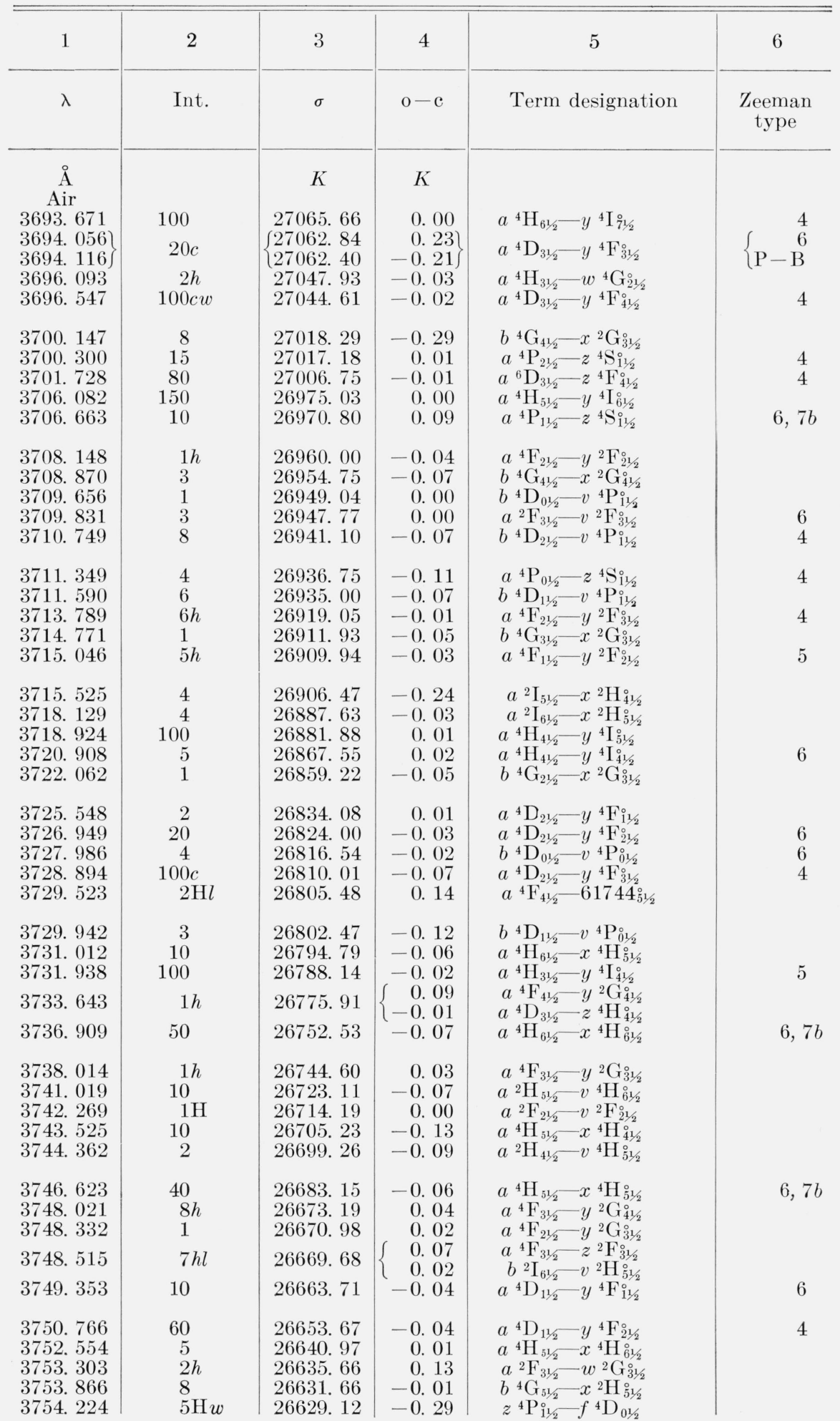


Table 1. Mn I-Classified lines - Continued

\begin{tabular}{|c|c|c|c|c|c|}
\hline 1 & 2 & 3 & 4 & 5 & 6 \\
\hline$\lambda$ & Int. & $\sigma$ & $\mathrm{o}-\mathrm{c}$ & Term designation & $\begin{array}{l}\text { Zeeman } \\
\text { type }\end{array}$ \\
\hline $\begin{array}{c}\AA \\
\text { Air } \\
3755.385 \\
3756.449 \\
3756.643 \\
3758.912 \\
3759.354\end{array}$ & $\begin{array}{l}1 \mathrm{H} w \\
5 \\
30 \\
4 h \\
2 h\end{array}$ & $\begin{array}{c}K \\
26620.89 \\
26613.35 \\
26611.98 \\
26595.91 \\
26592.79\end{array}$ & $\begin{array}{c}K \\
0.14 \\
0.04 \\
0.00 \\
-0.09 \\
0.11\end{array}$ & $\begin{array}{c}z^{4} \mathrm{P}_{21 / 2}^{\circ}-f^{4} \mathrm{D}_{11 / 2} \\
a^{4} \mathrm{H}_{41 / 2}-x \\
{ }^{4} \mathrm{H}_{31 / 2}^{\circ} \\
a^{4} \mathrm{H}_{41 / 2}-x{ }^{4} \mathrm{H}_{41 / 2}^{0} \\
a^{4} \mathrm{~F}_{21 / 2}-z^{2} \mathrm{~F}_{31 / 2}^{0} \\
a^{2} \mathrm{~F}_{31 / 2}-w^{2} \mathrm{G}_{41 / 2}^{2}\end{array}$ & \\
\hline $\begin{array}{l}3759.775 \\
3761.010 \\
3761.629 \\
3763.372 \\
3763.650\end{array}$ & $\begin{array}{l}6 \\
5 \mathrm{H} w \\
1 \\
40 \\
2 h\end{array}$ & $\begin{array}{l}26589.81 \\
26581.08 \\
26576.70 \\
26564.40 \\
26562.43\end{array}$ & $\begin{array}{r}-0.02 \\
0.20 \\
-0.04 \\
0.00 \\
0.02\end{array}$ & $\begin{array}{c}a{ }^{4} \mathrm{H}_{41 / 2}-x{ }^{4} \mathrm{H}_{51 / 2}^{\circ} \\
z^{4} \mathrm{P}_{01 / 2}^{\circ}-f^{4} \mathrm{D}_{01 / 2} \\
a^{2} \mathrm{H}_{51 / 2}-x{ }^{2} \mathrm{G}_{41 / 2}^{0} \\
a^{4} \mathrm{D}_{01 / 2}-y{ }^{4} \mathrm{~F}_{11 / 2}^{0} \\
a^{4} \mathrm{~F}_{11 / 2}-z^{2} \mathrm{~F}_{21 / 2}^{1}\end{array}$ & 5 \\
\hline $\begin{array}{l}3766.052 \\
3767.695 \\
3767.876 \\
3768.176 \\
3770.226\end{array}$ & $\begin{array}{l}10 \mathrm{H} \\
20 \\
2 \\
10 \\
1 \mathrm{H} ?\end{array}$ & $\begin{array}{l}26545.49 \\
26533.92 \\
26532.64 \\
26530.53 \\
26516.11\end{array}$ & $\begin{array}{r}0.01 \\
-0.02 \\
0.03 \\
0.02 \\
-0.15\end{array}$ & $\begin{array}{c}z^{4} \mathrm{P}_{11 / 2}^{\circ}-f^{4} \mathrm{D}_{11 / 2} \\
a^{4} \mathrm{H}_{31 / 2}-x \\
a^{4} \mathrm{H}_{31 / 2}^{\circ} \\
a^{4} \mathrm{H}_{31 / 2}-x \\
a^{4} \mathrm{H}_{41 / 2}^{\circ} \\
a^{4} \mathrm{~F}_{41 / 2}-w^{4} \mathrm{G}_{51 / 2}^{\circ} \\
a^{4} \mathrm{D}_{21 / 2}-z^{4} \mathrm{H}_{31 / 2}^{5}\end{array}$ & \\
\hline $\begin{array}{l}3771.439 \\
3772.955 \\
3773.858 \\
3774.669 \\
3776.289\end{array}$ & $\begin{array}{l}15 \\
10 \mathrm{H} \\
10 \\
20 h \\
2 h\end{array}$ & $\begin{array}{l}26507.58 \\
26496.93 \\
26490.58 \\
26484.89 \\
26473.53\end{array}$ & $\begin{array}{r}0.00 \\
-0.02 \\
0.00 \\
0.07 \\
0.11\end{array}$ & $\begin{array}{c}b{ }^{2} \mathrm{I}_{61 / 2}-x^{2} \mathrm{I}_{61 / 2}^{\circ} \\
z^{4} \mathrm{P}_{01 / 2}-f^{4} \mathrm{D}_{11 / 2} \\
b^{2} \mathrm{I}_{51 / 2}-x^{2} \mathrm{I}_{51 / 2}^{\circ} \\
z^{4} \mathrm{P}_{21 / 2}^{\circ}-f^{4} \mathrm{D}_{21 / 2}^{\circ} \\
a^{2} \mathrm{G}_{41 / 2}-w^{2} \mathrm{H}_{51 / 2}^{\circ}\end{array}$ & $\begin{array}{l}6,7 b \\
6,7 b\end{array}$ \\
\hline $\begin{array}{l}3776.537 \\
3781.192 \\
3783.297 \\
3784.233 \\
3785.422\end{array}$ & $\begin{array}{l}40 \\
1 h \\
2 \\
1 \\
30 h\end{array}$ & $\begin{array}{l}26471.79 \\
26439.20 \\
26424.49 \\
26417.96 \\
26409.66\end{array}$ & $\begin{array}{r}0.00 \\
-0.03 \\
-0.26 \\
-0.01 \\
0.11\end{array}$ & $\begin{array}{c}a{ }^{6} \mathrm{D}_{41 / 2}-z^{6} \mathrm{~F}_{31 / 2}^{\circ} \\
a{ }^{4} \mathrm{~F}_{31 / 2}-w^{4} \mathrm{G}_{31 / 2}^{\circ} \\
b^{4} \mathrm{G}_{41 / 2}-x^{2} \mathrm{H}_{41 / 2}^{0} \\
a^{4} \mathrm{H}_{51 / 2}-z^{2} \mathrm{G}_{41 / 2}^{0} \\
z^{4} \mathrm{P}_{11 / 2}^{11 / 2} f^{4} \mathrm{D}_{21 / 2}\end{array}$ & 4 \\
\hline $\begin{array}{l}3786.836 \\
3787.446 \\
3789.757 \\
3790.214 \\
3791.081\end{array}$ & $\begin{array}{r}3 \\
1 \\
1 h \\
200 h \\
2 h\end{array}$ & $\begin{array}{l}26399.80 \\
26395.55 \\
26379.45 \\
26376.27 \\
26370.22\end{array}$ & $\begin{array}{r}-0.03 \\
0.03 \\
-0.19 \\
-0.02 \\
0.03\end{array}$ & $\begin{array}{c}a{ }^{2} \mathrm{I}_{51 / 2}-z^{2} \mathrm{H}_{41 / 2}^{0} \\
a^{4} \mathrm{H}_{41 / 2}-z^{2} \mathrm{G}_{31 / 2}^{0} \\
a^{4} \mathrm{D}_{11 / 2}-v \\
{ }^{6} \mathrm{P}_{11 / 2}^{0} \\
a^{6} \mathrm{D}_{41 / 2}-z^{6} \mathrm{~F}_{41 / 2}^{0} \\
a^{2} \mathrm{~F}_{21 / 2}-w^{2} \mathrm{G}_{31 / 2}^{11}\end{array}$ & $7 b$ \\
\hline $\begin{array}{l}3794.497 \\
3799.256 \\
3800.551 \\
3801.901 \\
3802.137\end{array}$ & $\begin{array}{l}2 \\
60 \\
60 h \\
80 \\
1\end{array}$ & $\begin{array}{l}26346.50 \\
26313.50 \\
26304.53 \\
26295.19 \\
26293.56\end{array}$ & $\begin{array}{r}-0.04 \\
0.00 \\
0.06 \\
0.00 \\
-0.04\end{array}$ & $\begin{array}{c}a^{2} \mathrm{G}_{31 / 2}-w^{2} \mathrm{H}_{41 / 2}^{\circ} \\
a^{6} \mathrm{D}_{31 / 2}-z^{6} \mathrm{~F}_{21 / 2}^{\circ} \\
z^{4} \mathrm{P}_{21 / 2}^{\circ}-f^{4} \mathrm{D}_{31 / 2} \\
a^{4} \mathrm{G}_{51 / 2}-z^{4} \mathrm{G}_{51 / 2}^{\circ} \\
a^{2} \mathrm{I}_{61 / 2}-w^{4} \mathrm{H}_{51 / 2}^{\circ}\end{array}$ & $\begin{array}{l}5 \\
6, \quad 7 b\end{array}$ \\
\hline $\begin{array}{l}\text { 3803. } 073 \\
3804.021 \\
3804.752 \\
3806.715 \\
3806.881\end{array}$ & $\begin{array}{c}2 \\
6 \\
20 c \\
2000 h \\
10\end{array}$ & $\begin{array}{l}26287.09 \\
26280.54 \\
26275.49 \\
26261.94 \\
26260.80\end{array}$ & $\begin{array}{r}0.02 \\
0.01 \\
-0.01 \\
0.00 \\
-0.04\end{array}$ & $\begin{array}{l}a{ }^{4} \mathrm{~F}_{41 / 2}-y^{4} \mathrm{I}_{51 / 2}^{0}{ }^{\circ}{ }^{4} \mathrm{G}_{51 / 2}-z^{4} \mathrm{G}_{41 / 2}^{\circ} \\
a^{4} \mathrm{G}_{41 / 2}-z^{4} \mathrm{G}_{51 / 2}^{\circ} \\
a^{6} \mathrm{D}_{41 / 2}-z^{6} \mathrm{~F}_{51 / 2}^{0} \\
a^{4} \mathrm{G}_{41 / 2}-z^{4} \mathrm{G}_{41 / 2}^{011}\end{array}$ & 4 \\
\hline $\begin{array}{l}3807.203 \\
3808.506 \\
3809.146 \\
3809.485 \\
3809.593\end{array}$ & $\begin{array}{c}10 \\
10 \\
5 \\
10 \\
500 h\end{array}$ & $\begin{array}{l}26258.58 \\
26249.59 \\
26245.18 \\
26242.85 \\
26242.10\end{array}$ & $\begin{array}{r}0.05 \\
0.02 \\
0.00 \\
-0.02 \\
0.02\end{array}$ & $\begin{array}{l}a^{4} \mathrm{G}_{31 / 2} z^{4} \mathrm{G}_{41 / 2}^{\circ} \\
a^{4} \mathrm{G}_{21 / 2} z^{4} \mathrm{G}_{31 / 2}^{\circ} \\
a^{4} \mathrm{G}_{41 / 2} z^{4} \mathrm{G}_{31 / 2}^{\circ} \\
a^{4} \mathrm{G}_{31 / 2} z^{4} \mathrm{G}_{31 / 2}^{\circ} \\
a^{6} \mathrm{D}_{31 / 2} z^{6} \mathrm{~F}_{31 / 2}^{\circ}\end{array}$ & 6 \\
\hline $\begin{array}{l}3810.679 \\
3811.659 \\
3813.024 \\
3816.746 \\
3820.081\end{array}$ & $\begin{array}{r}40 \\
6 \\
1 \\
100 \\
1 h\end{array}$ & $\begin{array}{l}26234.62 \\
26227.88 \\
26218.49 \\
26192.92 \\
26170.06\end{array}$ & $\begin{array}{r}0.03 \\
-0.01 \\
0.09 \\
-0.01 \\
0.00\end{array}$ & 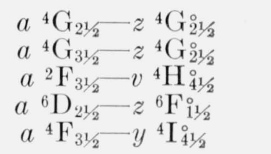 & $5, \mathrm{C}$ \\
\hline $\begin{array}{l}3820.903 \\
3823.508 \\
3823.891 \\
3826.617 \\
3826.734\end{array}$ & $\begin{array}{r}1 h \\
1500 h \\
100 h \\
5 h \\
1 h\end{array}$ & $\begin{array}{l}26164.42 \\
26146.60 \\
26143.98 \\
26125.36 \\
26124.55\end{array}$ & $\begin{array}{l}0.14 \\
0.02 \\
0.00 \\
0.00 \\
0.02\end{array}$ & $\begin{array}{c}b^{4} \mathrm{P}_{21 / 2}-w^{4} \mathrm{D}_{11 / 2}^{\circ} \\
a^{6} \mathrm{D}_{31 / 2}-z^{6} \mathrm{~F}_{41 / 2}^{\circ} \\
a^{6}{ }^{6} \mathrm{D}_{21 / 2}-z^{6} \mathrm{~F}_{21 / 2}^{\circ} \\
z^{6} \mathrm{P}_{11 / 2}^{\circ} g^{6} \mathrm{~S}_{21 / 2}^{\circ} \\
a^{2} \mathrm{I}_{61 / 2}-z^{2} \mathrm{H}_{51 / 2}^{2}\end{array}$ & $6, \stackrel{4}{\mathrm{C}}$ \\
\hline
\end{tabular}


TABle 1. Mn I-Classified lines-Continued

\begin{tabular}{|c|c|c|c|c|c|}
\hline 1 & 2 & 3 & 4 & 5 & 6 \\
\hline$\lambda$ & Int. & $\sigma$ & $o-c$ & Term designation & $\begin{array}{c}\text { Zeeman } \\
\text { type }\end{array}$ \\
\hline $\begin{array}{c}\AA \\
\text { Air } \\
3827.890 \\
3829.679 \\
3829.986 \\
3833.865 \\
3834.368\end{array}$ & $\begin{array}{c}10 \mathrm{H} \\
100 \\
8 \mathrm{~h} \\
500 \\
1000\end{array}$ & $\begin{array}{c}K \\
26116.67 \\
26104.47 \\
26102.38 \\
26075.97 \\
26072.55\end{array}$ & $\begin{array}{r}K \\
0.04 \\
0.29 \\
-0.05 \\
0.00 \\
-0.01\end{array}$ & 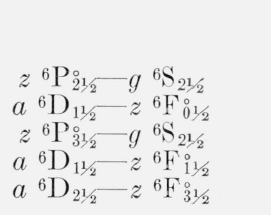 & $\begin{array}{r}5, \mathrm{C} \\
6, \mathrm{C} \\
4\end{array}$ \\
\hline $\begin{array}{l}\text { 3836. } 908 \\
3838.191 \\
3839.779 \\
3841.074 \\
3843.988\end{array}$ & $\begin{array}{l}1 h \\
2 h l \\
500 h \\
600 h \\
500 h\end{array}$ & $\begin{array}{l}26055.29 \\
26046.58 \\
26035.81 \\
26027.03 \\
26007.30\end{array}$ & $\begin{array}{r}-0.10 \\
-0.09 \\
0.30 \\
0.01 \\
0.00\end{array}$ & $\begin{array}{l}b^{4} \mathrm{G}_{21 / 2}-x^{2} \mathrm{D}_{11 / 2}^{\circ} \\
a^{2} \mathrm{H}_{51 / 2}-x^{2} \mathrm{H}_{41 / 2}^{0} \\
a^{6} \mathrm{D}_{01 / 2}-z^{6} \mathrm{~F}_{01 / 2}^{0} \\
a^{6} \mathrm{D}_{11 / 2}-z^{6} \mathrm{~F}_{21 / 2}^{\circ} \\
a^{6} \mathrm{D}_{01 / 2}-z^{6} \mathrm{~F}_{11 / 2}^{0}\end{array}$ & $\begin{array}{l}6, \mathrm{C} \\
4, \mathrm{C} \\
4, \mathrm{C}\end{array}$ \\
\hline $\begin{array}{l}3845.009 \\
3845.795 \\
3847.234 \\
3846.046 \\
3850.380\end{array}$ & $\begin{array}{l}7 h \\
1 \\
1 h \\
1 \\
1 h\end{array}$ & $\begin{array}{l}26000.40 \\
25995.08 \\
25985.36 \\
25979.88 \\
25964.12\end{array}$ & $\begin{array}{r}0.13 \\
0.05 \\
-0.03 \\
0.28 \\
0.02\end{array}$ & $\begin{array}{l}a^{4} \mathrm{~F}_{41 / 2}-w^{4} \mathrm{~F}_{41 / 2}^{\circ} \\
a^{4} \mathrm{~F}_{41 / 2}-x^{4} \mathrm{H}_{51 / 2}^{\circ} \\
a^{2} \mathrm{~F}_{21 / 2}-v^{4} \mathrm{H}_{31 / 2}^{\circ} \\
a^{2} \mathrm{~F}_{31 / 2}-x^{2} \mathrm{G}_{31 / 2}^{\circ} \\
a^{4} \mathrm{~F}_{41 / 2}-w^{4} \mathrm{~F}_{31 / 2}^{0}\end{array}$ & \\
\hline $\begin{array}{l}3852.512 \\
3853.476 \\
3854.674 \\
3855.114 \\
3856.539\end{array}$ & $\begin{array}{l}6 h \\
30 \\
1 \\
4 h \\
50\end{array}$ & $\begin{array}{l}\text { 25949. } 76 \\
25943.27 \\
25935.17 \\
25932.24 \\
25922.66\end{array}$ & $\begin{array}{r}-0.06 \\
-0.03 \\
-0.02 \\
-0.03 \\
0.11\end{array}$ & $\begin{array}{l}a^{2} \mathrm{G}_{41 / 2}-66981_{31 / 2}^{\circ} \\
b{ }^{4} \mathrm{G}_{51 / 2}-w^{4} \mathrm{H}_{61 / 2}^{\circ} \\
a^{2} \mathrm{H}_{41 / 2}-x^{2} \mathrm{H}_{11 / 2}^{\circ} \\
b^{4} \mathrm{P}_{11 / 2}-z^{2} \mathrm{D}_{11 / 2}^{\circ} \\
a^{4} \mathrm{P}_{21 / 2}-y^{4} \mathrm{D}_{31 / 2}^{\circ}\end{array}$ & 4 \\
\hline $\begin{array}{l}3857.280 \\
3857.563 \\
3857.753 \\
3858.749 \\
3859.397\end{array}$ & $\begin{array}{l}1 \\
1 h \\
1 \\
5 \\
1\end{array}$ & $\begin{array}{l}25917.68 \\
25915.78 \\
25914.51 \\
25907.82 \\
25903.47\end{array}$ & $\left\{\begin{array}{r}-0.19 \\
-0.06 \\
-0.06 \\
0.00 \\
0.15 \\
0.03\end{array}\right.$ & $\begin{array}{l}b{ }^{4} \mathrm{G}_{41 / 2}-z^{2} \mathrm{H}_{41 / 2}^{0} \\
a^{4} \mathrm{~F}_{31 / 2}-x{ }^{4} \mathrm{H}_{31 / 2}^{\circ} \\
a^{2} \mathrm{~F}_{31 / 2}-x{ }^{2} \mathrm{G}_{41 / 2}^{01 / 2} \\
a^{4} \mathrm{~F}_{31 / 2}-x{ }^{4} \mathrm{H}_{41 / 2}^{\circ} \\
a^{4} \mathrm{P}_{21 / 2}-y^{4} \mathrm{D}_{21 / 2}^{\circ} \\
a^{4} \mathrm{G}_{51 / 2}-x{ }^{6} \mathrm{~F}_{51 / 2}^{1}\end{array}$ & \\
\hline $\begin{array}{l}3860.258 \\
3862.320 \\
3865.672 \\
3866.574 \\
3868.535\end{array}$ & $\begin{array}{c}3 h \\
1 h \\
20 \\
2 \\
1\end{array}$ & $\begin{array}{l}25897.69 \\
25883.86 \\
25861.42 \\
25855.39 \\
25842.28\end{array}$ & $\begin{array}{l}0.09 \\
0.11 \\
0.21 \\
0.20 \\
0.05\end{array}$ & $\begin{array}{l}a^{4} \mathrm{~F}_{31 / 2}-w^{4} \mathrm{~F}_{41 / 2}^{\circ} \\
a^{4} \mathrm{G}_{41 / 2}-x^{6} \mathrm{~F}_{51 / 2}^{\circ} \\
a^{4} \mathrm{P}_{11 / 2}-y^{4} \mathrm{D}_{21 / 2}^{\circ} \\
a^{4} \mathrm{P}_{11 / 2}-y^{4} \mathrm{D}_{11 / 2}^{\circ} \\
a^{4} \mathrm{~F}_{21 / 2}-x^{4} \mathrm{H}_{31 / 2}^{0}\end{array}$ & \\
\hline $\begin{array}{l}3870.822 \\
3871.345 \\
3871.671 \\
2871.953 \\
3872.127\end{array}$ & $\begin{array}{l}4 \\
1 \mathrm{H} \\
6 h \\
2 \\
10\end{array}$ & $\begin{array}{l}25827.01 \\
25823.52 \\
25821.34 \\
25819.47 \\
25818.31\end{array}$ & $\begin{array}{r}-0.22 \\
0.00 \\
0.00 \\
0.00 \\
-0.20\end{array}$ & $\begin{array}{l}b^{4} \mathrm{G}_{41 / 2}-w^{4} \mathrm{H}_{51 / 2}^{\circ} \\
a^{2} \mathrm{G}_{41 / 2}-u^{4} \mathrm{~F}_{41 / 2}^{\circ} \\
a^{4} \mathrm{P}_{01 / 2}-y{ }^{4} \mathrm{D}_{11 / 2}^{\circ} \\
a^{4} \mathrm{P}_{01 / 2}-y{ }^{4} \mathrm{D}_{01 / 2}^{\circ} \\
b^{4} \mathrm{G}_{41 / 2}-y^{2} \mathrm{H}_{51 / 2}^{0}\end{array}$ & \\
\hline $\begin{array}{l}\text { 3873. } 200 \\
3874.743 \\
3876.712 \\
\text { 3878. } 155 \\
\text { 3883. } 249\end{array}$ & $\begin{array}{l}10 \\
1 h \\
3 \\
5 h \\
20\end{array}$ & $\begin{array}{l}25811.16 \\
25800.87 \\
25787.77 \\
25778.18 \\
25744.36\end{array}$ & $\begin{array}{r}-0.11 \\
0.15 \\
-0.05 \\
-0.06 \\
0.10\end{array}$ & 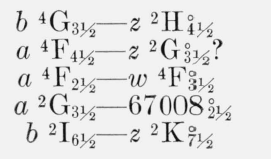 & 4 \\
\hline $\begin{array}{l}\text { 3887. } 380 \\
3888.841 \\
3889.461 \\
3891.624 \\
3892.621\end{array}$ & $\begin{array}{r}2 \\
7 \\
20 \\
5 \\
15\end{array}$ & $\begin{array}{l}25717.00 \\
25707.35 \\
25703.25 \\
25688.96 \\
25682.38\end{array}$ & $\begin{array}{r}-0.29 \\
-0.04 \\
0.00 \\
-004 \\
0.05\end{array}$ & $\begin{array}{l}b{ }^{4} \mathrm{G}_{41 / 2}-y^{2} \mathrm{H}_{41 / 2}^{\circ} \\
b^{4} \mathrm{G}_{31 / 2}-w^{4} \mathrm{H}_{41 / 2}^{\circ} \\
b^{2} \mathrm{I}_{51 / 2}-z^{2} \mathrm{~K}_{61 / 2}^{\circ} \\
a^{4} \mathrm{H}_{61 / 2}-z^{2} \mathrm{I}_{51 / 2}^{\circ} \\
a^{4} \mathrm{P}_{21 / 2}-x^{6} \mathrm{D}_{21 / 2,1 / 2}^{\circ}\end{array}$ & 5 \\
\hline $\begin{array}{l}\text { 3893. } 199 \\
\text { 3894. } 714\end{array}$ & $\begin{array}{r}1 \\
60\end{array}$ & $\begin{array}{l}25678.57 \\
25668.58\end{array}$ & $\begin{array}{r}-0.04 \\
0.02\end{array}$ & $\begin{array}{l}b^{4} \mathrm{P}_{11 / 2}-w^{4} \mathrm{D}_{01 / 2}^{\circ} \\
a^{4} \mathrm{P}_{21 / 2}-x^{6} \mathrm{D}_{31 / 2}^{\circ}\end{array}$ & 4 \\
\hline $\begin{array}{l}3896.743 \\
3898.368\end{array}$ & $\begin{array}{r}2 h \\
50 c\end{array}$ & $\begin{array}{l}25657.87 \\
25655.21 \\
25644.52\end{array}$ & $\left\{\begin{array}{l}-0.29 \\
-0.29 \\
-0.10 \\
-0.09 \\
-0.13\end{array}\right.$ & $\begin{array}{l}b^{4} \mathrm{G}_{41 / 2}-z^{2} \mathrm{H}_{51 / 2}^{\circ} \\
b^{4} \mathrm{G}_{41 / 2}-x{ }^{2} \mathrm{~F}_{31 / 2} \\
b^{4} \mathrm{P}_{21 / 2}-v{ }^{4} \mathrm{D}_{21 / 2}^{\circ} \\
a^{4} \mathrm{~F}_{11 / 2}-w^{4} \mathrm{~F}_{21 / 2}^{o} \\
b^{4} \mathrm{P}_{21 / 2}-v{ }^{4} \mathrm{D}_{31 / 2}^{0}\end{array}$ & 4 \\
\hline
\end{tabular}


Table 1. Mn I-Classified lines-Continued

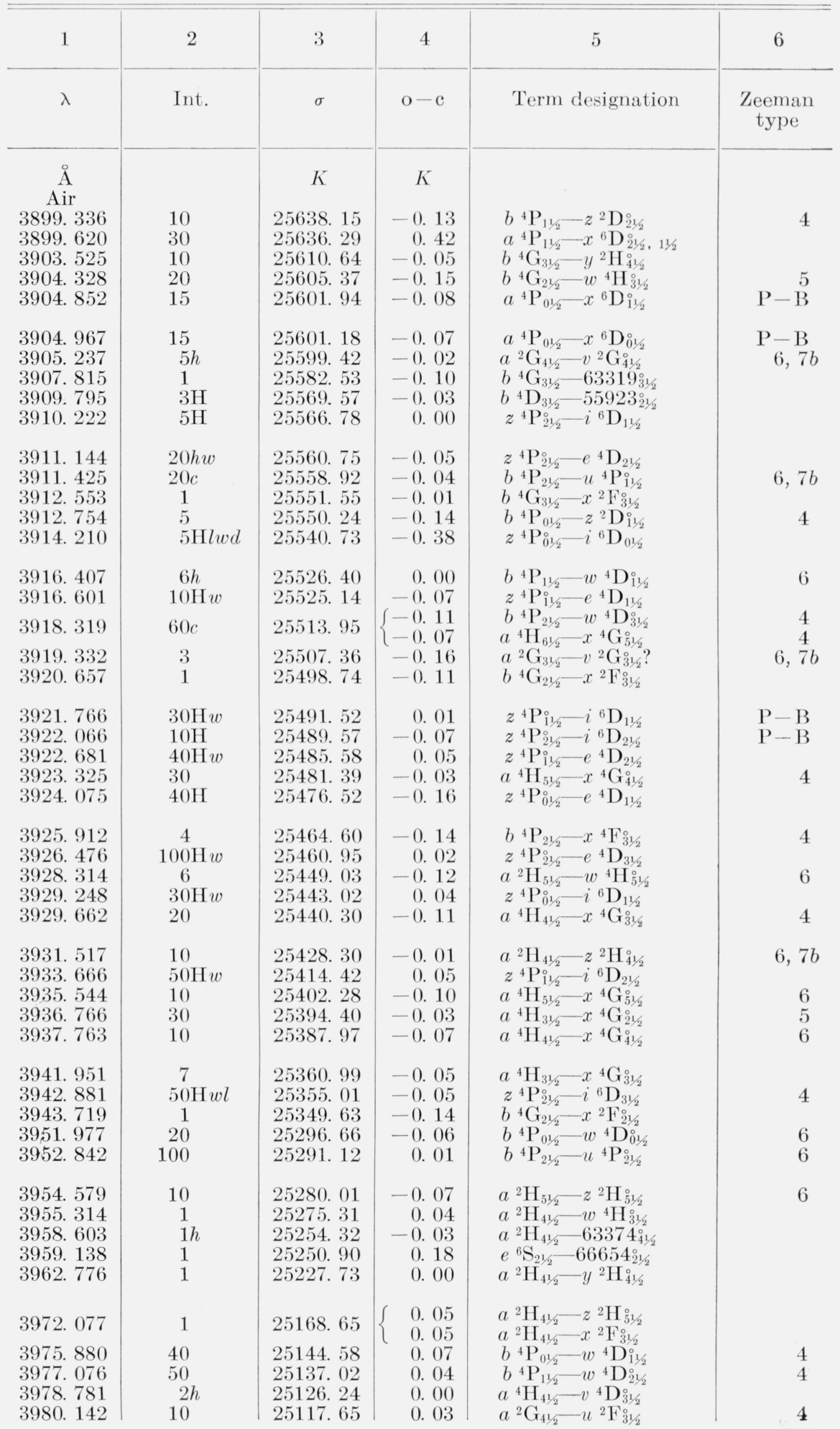


TaBle 1. Mn I-Classified lines-Continued

\begin{tabular}{|c|c|c|c|c|c|}
\hline 1 & 2 & 3 & 4 & 5 & 6 \\
\hline$\lambda$ & Int. & $\sigma$ & $\mathrm{o}-\mathrm{c}$ & Term designation & $\begin{array}{l}\text { Zeeman } \\
\text { type }\end{array}$ \\
\hline $\begin{array}{r}\AA \\
\text { Air }\end{array}$ & & $K$ & $K$ & & \\
\hline 3982. 164 & 30 & 25104. 90 & -0.02 & $b^{4} \mathrm{P}_{11 / 2}-u{ }^{4} \mathrm{P}_{01 / 2}^{\circ}$ & \\
\hline 3982. 576 & 100 & 25102. 30 & 0.07 & $a^{4} \mathrm{G}_{21 / 2}^{11 / 2}-y^{4} \mathrm{~F}_{11 / 2}^{01 / 2}$ & $\mathrm{P}-\mathrm{B}$ \\
\hline 3982. 900 & 40 & 25100.26 & -0.02 & $e^{6} \mathrm{~S}_{21 / 2}-66504_{11 / 2}^{2}$ & \\
\hline $\begin{array}{l}\text { 3984. } 172 \\
3985.236\end{array}$ & $\begin{array}{r}20 \\
100\end{array}$ & $\begin{array}{l}25092.25 \\
25085.55\end{array}$ & $\begin{array}{l}0.06 \\
0.06\end{array}$ & $a^{4} \mathrm{G}_{21 / 2}-y^{4} \mathrm{~F}_{21 / 2}^{\circ}$ & $P-B$ \\
\hline 3986. 377 & 2 & 25078. 34 & 0.10 & $a^{4} \mathrm{G}_{21 / 2}-y^{4} \mathrm{~F}_{31 / 1}^{\circ}$ & \\
\hline 3986. 822 & 200 & 25075.57 & 0.01 & $a^{4} \mathrm{G}_{51 / 2}^{21 / 2}-y^{4} \mathrm{~F}_{41 / 2}^{31 / 2}$ & 4 \\
\hline 3987. 092 & 100 & 25073.87 & 0.02 & $a^{4} \mathrm{G}_{41 / 2}-y^{4} \mathrm{~F}_{31 / 2}^{0}$ & . 4 \\
\hline 3987.455 & 20 & 25071.59 & 0.05 & $a^{4} \mathrm{G}_{31 / 2}-y^{4} \mathrm{~F}_{31 / 2}^{0}$ & $\mathrm{P}-\mathrm{B}$ \\
\hline 3988. 666 & 8 & 25063. 98 & -0.01 & $b{ }^{4} \mathrm{P}_{11 / 2}-v{ }^{4} \mathrm{D}_{01 / 2}^{0}$ & $\mathrm{P}-\mathrm{B}$ \\
\hline 3989. 699 & 1 & 25057.49 & -0.04 & $a{ }^{4} \mathrm{H}_{31 / 2}-v^{4} \mathrm{D}_{21 / 2}^{\circ}$ & \\
\hline 3989. 952 & 30 & 25055.90 & 0.03 & $a^{4} \mathrm{G}_{41 / 2}-y{ }^{4} \mathrm{~F}_{41 / 2}^{21 / 2}$ & 6 \\
\hline 3990.737 & 1 & 25050.97 & 0.04 & $b^{4} \mathrm{D}_{31 / 2}-w^{4} \mathrm{P}_{21 / 2}^{\circ}$ & \\
\hline $\begin{array}{l}3991.596 \\
3993.858\end{array}$ & $\begin{array}{r}20 \\
6\end{array}$ & $\begin{array}{l}25045.58 \\
25031.40\end{array}$ & $\begin{array}{r}0.12 \\
-0.09\end{array}$ & 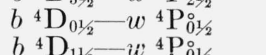 & $\mathrm{P}-\mathrm{B}$ \\
\hline & & & & 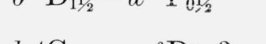 & \\
\hline $\begin{array}{l}\text { 3995. } 012 \\
3996.101\end{array}$ & $1 h$ & 25024.17 & 0. 06 & $b^{4} \mathrm{G}_{31 / 2}-w^{6} \mathrm{D}_{21 / 2}^{\circ} ?$ & \\
\hline $\begin{array}{l}\text { 3996. } 101 \\
3997.771\end{array}$ & $\begin{array}{l}5 \mathrm{H} s \\
3 h\end{array}$ & 25017. 34 & $\begin{array}{l}-0.09 \\
-0.03\end{array}$ & & 4 \\
\hline $\begin{array}{l}3997.771 \\
3999.573\end{array}$ & $\begin{array}{l}3 n \\
1 h\end{array}$ & $\begin{array}{l}25006.89 \\
24995.63\end{array}$ & $\begin{array}{l}-0.03 \\
-0.02\end{array}$ & $\begin{array}{l}a^{4} \mathrm{H}_{51 / 2}-x^{4} \mathrm{~F}_{41 / 2}^{1} \\
a^{4} \mathrm{H}_{41}-w^{4} \mathrm{D}_{31}^{2}\end{array}$ & \\
\hline 4001. 190 & 15 & 24985. 52 & -0.01 & $b^{4} \mathrm{D}_{21 / 2}-w^{4} \mathrm{P}_{21 / 2}^{301 / 2}$ & $\mathrm{P}-\mathrm{B}$ \\
\hline 4002. 169 & 15 & 24979.42 & -0.01 & $b^{4} \mathrm{D}_{11 / 2}-w^{4} \mathrm{P}_{21 / 2}^{0}$ & $\mathrm{P}-\mathrm{B}$ \\
\hline 4002.559 & 1 & 24976.98 & 0.05 & $a^{4} \mathrm{~F}_{11 / 2}-w^{4} \mathrm{D}_{01 / 2}^{\circ}$ & \\
\hline 4003. 258 & $20 \mathrm{Hl}$ & 24972.63 & 0.05 & $b^{4} \mathrm{G}_{51 / 2}^{1 / 2}-v{ }^{4} \mathrm{~F}_{41 / 2}^{0}$ & \\
\hline 4005. 775 & 1 & 24956. 93 & 0. 01 & $b{ }^{4} \mathrm{D}_{01 / 2}-w^{4} \mathrm{P}_{11 / 2}^{0}$ & \\
\hline 4007. 041 & 10 & 24949. 04 & -0.01 & $b{ }^{4} \mathrm{D}_{21 / 2}-w^{4} \mathrm{P}_{11 / 2}^{112}$ & $\mathrm{P}-\mathrm{B}$ \\
\hline 4008. 022 & 20 & 24942.94 & -0.01 & $b^{4} \mathrm{D}_{11 / 2}-w^{4} \mathrm{P}_{11 / 2}^{\circ}$ & $\mathrm{P}-\mathrm{B}$ \\
\hline 4011. 535 & 30 & 24921. 09 & 0.01 & $b{ }^{4} \mathrm{P}_{11 / 2}-u{ }^{4} \mathrm{P}_{11 / 2}^{0}$ & 6 \\
\hline 4011. 913 & $10 h$ & 24918. 75 & -0.05 & $a^{2} \mathrm{G}_{31 / 2}-u^{2} \mathrm{~F}_{31 / 2}^{\circ}$ & 6 \\
\hline $\begin{array}{l}\text { 4012. } 761 \\
4016.671\end{array}$ & $\begin{array}{l}2 \\
5\end{array}$ & $\begin{array}{l}24913.48 \\
24889.23\end{array}$ & $\begin{array}{r}-0.06 \\
0.05\end{array}$ & $\begin{array}{c}a^{4} \mathrm{H}_{41 / 2}-x^{4} \mathrm{~F}_{41 / 2}^{\circ} \\
a^{4} \mathrm{~F}_{41 / 2}-z^{2} \mathrm{I}_{51 / 2}^{\circ}\end{array}$ & 4 \\
\hline 4018. 106 & $1000 h$ & 24880.34 & -0.01 & $a^{6} \mathrm{D}_{41 / 2}-z^{6} \mathrm{D}_{31 / 2}^{\circ}$ & 4 \\
\hline 4018.583 & $2 h$ & 24877. 39 & -0.05 & $a^{2} \mathrm{G}_{41 / 2}^{1 / 2}-v^{4} \mathrm{G}_{41 / 2}^{072}$ & \\
\hline 4020. 072 & 10 & 24868.17 & 0.00 & $b{ }^{4} \mathrm{D}_{01 / 2}-x{ }^{4} \mathrm{D}_{11 / 2}^{\circ}$ & 5 \\
\hline 4021. 354 & 2 & 24860.25 & -0.05 & $b{ }^{4} \mathrm{D}_{21 / 2}-x{ }^{4} \mathrm{D}_{11 / 2}$ & \\
\hline 4022.335 & $2 h$ & 24854. 18 & $\left\{\begin{array}{l}-0.02 \\
-0.04\end{array}\right.$ & $\begin{array}{l}b^{4} \mathrm{D}_{11 / 2}-x^{4} \mathrm{D}_{11 / 2}^{\circ} \\
a^{2} \mathrm{~F}_{31 / 2}-63523_{21 / 2}^{\circ}\end{array}$ & \\
\hline 4023. 719 & 1 & 24845. 63 & 0.02 & $a^{4} \mathrm{~F}_{41 / 2}-x^{4} \mathrm{G}_{31 / 2}^{\circ}$ & \\
\hline 4025. 938 & 4 & 24831. 94 & -0.02 & $b{ }^{4} \mathrm{D}_{31 / 2}^{4 / 2}-x{ }^{4} \mathrm{D}_{21 / 2}^{0}$ & 5 \\
\hline 4026. 437 & 80 & 24828. 86 & 0.00 & $a^{4} \mathrm{G}_{51 / 2}-z^{4} \mathrm{H}_{61 / 2}$ & 4 \\
\hline 4028.595 & $5 \mathrm{H}$ & 24815.56 & -0.01 & $a^{4} \mathrm{G}_{51 / 2}-z^{4} \mathrm{H}_{51 / 2}^{0}$ & 6 \\
\hline 4030. 755 & $20000 \mathrm{R} w$ & 24802. 26 & 0.01 & $a^{6} \mathrm{~S}_{21 / 2}-z^{6} \mathrm{P}_{31 / 2}^{0}$ & 4 \\
\hline 4031. 791 & 100 & 24795. 89 & 0.01 & $a^{4} \mathrm{G}_{41 / 2}-z^{4} \mathrm{H}_{51 / 2}^{\circ}$ & \\
\hline 4033. 068 & $15000 \mathrm{R} w$ & 24788.04 & -0.01 & $a^{6} \mathrm{~S}_{21 / 2}-z^{6} \mathrm{P}_{21 / 2}^{0}$ & 6 \\
\hline 4033. 587 & 4 & 24784.85 & 0.00 & $a^{4} \mathrm{G}_{31 / 2}-z^{4} \mathrm{H}_{41 / 2}^{2}$ & \\
\hline 4033. 652 & 3 & 24784.45 & 0.03 & $a^{4} \mathrm{G}_{21 / 2}-z^{4} \mathrm{H}_{31 / 2}^{0}$ & \\
\hline 4034. 485 & $10000 \mathrm{R} w$ & 24779. 33 & 0.01 & $a^{6} \mathrm{~S}_{21 / 2}-z^{6} \mathrm{P}_{11 / 2}^{0}$ & 4 \\
\hline 4035. 729 & 1000 & 24771. 70 & -0.03 & $a^{6} \mathrm{D}_{31 / 2}-z^{6} \mathrm{D}_{21 / 2}^{\circ}$ & 4 \\
\hline 4036. 244 & 4 & 24768.51 & -0.22 & $a^{2} \mathrm{P}_{11 / 2}-z^{2} \mathrm{P}_{11 / 2}^{01 / 2}$ & \\
\hline 4036. 562 & $\begin{array}{l}5 \\
5\end{array}$ & 24766.58 & 0.02 & $b^{4} \mathrm{D}_{21 / 2}^{1 / 2}-x^{4} \mathrm{D}_{21 / 2}^{\circ}$ & \\
\hline $\begin{array}{l}4037.561 \\
4038.728\end{array}$ & $\begin{array}{r}5 \\
20\end{array}$ & $\begin{array}{l}24760.45 \\
24753.30\end{array}$ & $\begin{array}{l}-0.01 \\
-0.01\end{array}$ & $\begin{array}{l}b{ }^{4} \mathrm{D}_{11 / 2}-x^{4} \mathrm{D}_{21 / 2}^{\circ} \\
b^{4} \mathrm{D}_{31 / 2}-x^{4} \mathrm{D}_{3116}^{\circ}\end{array}$ & $6,7 b$ \\
\hline 4039.250 & $1 \mathrm{Hl}$ & 24750.10 & -0.04 & $b^{4} \mathrm{G}_{31}-v^{4} \mathrm{~F}_{: 1}^{\circ}$ & \\
\hline 4040.424 & & 24742.95 & 0.01 & $a^{4} \mathrm{~F}_{31-x}-{ }^{4} \mathrm{G}^{21 / 2}$ & \\
\hline 4041.357 & $2000 h$ & 24737. 20 & 0.01 & $a^{6} \mathrm{D}_{41 / 2}^{3 / 2}-z^{6} \mathrm{D}_{41 / 2}^{\circ 1 / 2}$ & $6,7 b$ \\
\hline 4043. 682 & $1 h$ & 24722.98 & -0.05 & $b^{4} \mathrm{P}_{01 / 2}-u{ }^{4} \mathrm{P}_{01 / 2}$ & \\
\hline 4045.115 & 50 & 24714. 22 & 0.02 & $a^{4} \mathrm{~F}_{41 / 2}^{0 / 2}-x^{4} \mathrm{G}_{51 / 2}^{01 / 2}$ & 4 \\
\hline
\end{tabular}


Table 1. Mn I-Classified lines-Continued

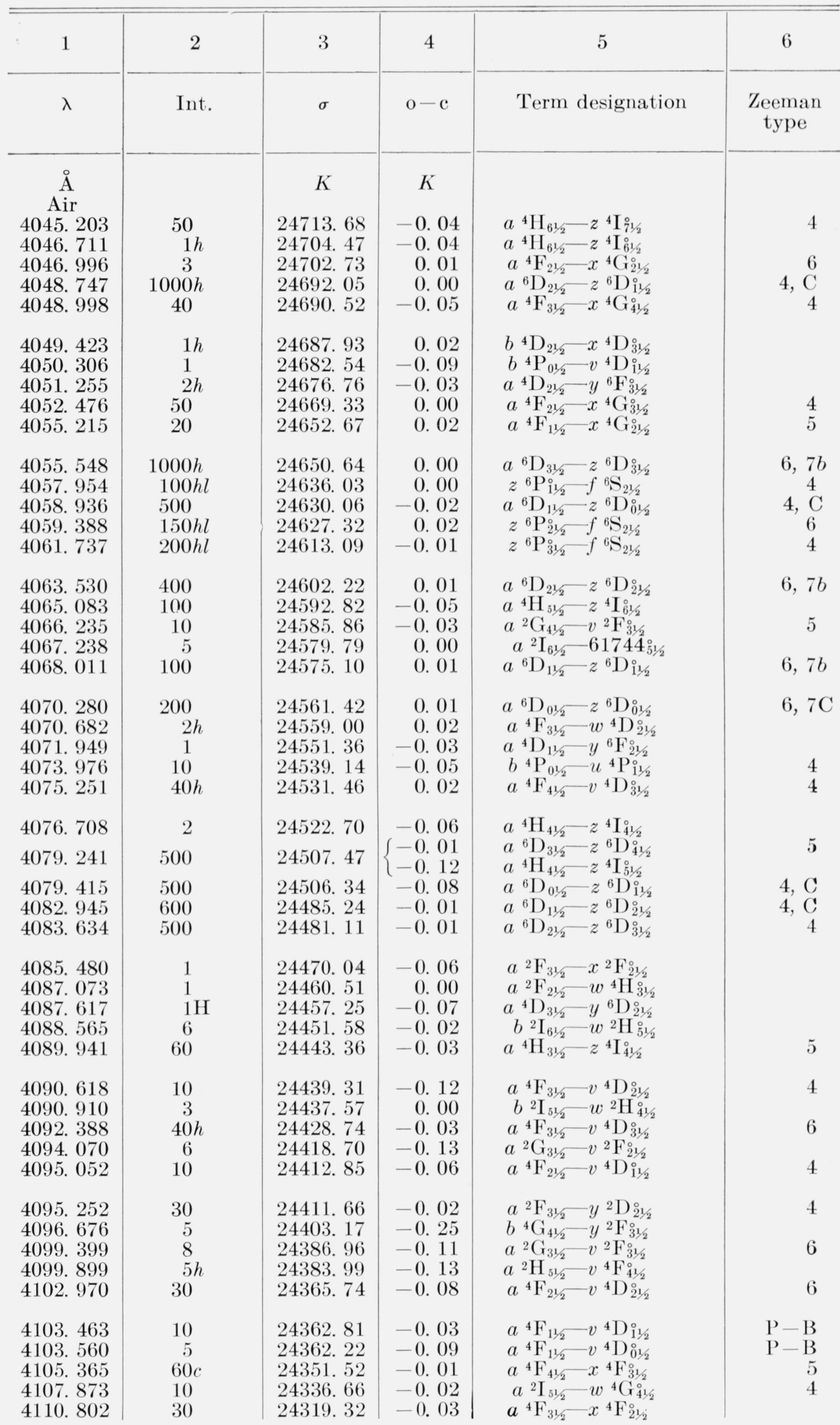


Table 1. Mn I-Classified lines-Continued

\begin{tabular}{|c|c|c|c|c|c|}
\hline 1 & 2 & 3 & 4 & 5 & 6 \\
\hline$\lambda$ & Int. & $\sigma$ & $\mathrm{o}-\mathrm{c}$ & Term designation & $\begin{array}{l}\text { Zeeman } \\
\text { type }\end{array}$ \\
\hline$\stackrel{\AA}{\AA}$ & & $K$ & K & & \\
\hline $\begin{array}{l}\text { 4110. } 894 \\
4111.416\end{array}$ & $\begin{array}{c}60 c \\
2\end{array}$ & $\begin{array}{l}24318.77 \\
24315.68\end{array}$ & $\begin{array}{r}0.03 \\
-0.07\end{array}$ & $\begin{array}{l}a^{4} \mathrm{~F}_{41 / 2}-x^{4} \mathrm{~F}_{41 / 2}^{\circ} \\
a^{4} \mathrm{~F}_{11 / 2}-v^{4} \mathrm{D}_{21 / 2}^{\circ}\end{array}$ & 6,7 \\
\hline 4113. 243 & 30 & 24304. 88 & $\left\{\begin{array}{r}0.10 \\
-0.08\end{array}\right.$ & $a^{4} \mathrm{P}_{11 / 2}^{1 / 2}-x^{4} \mathrm{P}_{01 / 2}^{21 / 2}$ & 4 \\
\hline $\begin{array}{l}\text { 4113. } 880 \\
4114.381\end{array}$ & $\begin{array}{l}20 \\
25\end{array}$ & $\begin{array}{l}\text { 24301. } 12 \\
24298.16\end{array}$ & $\begin{array}{l}-0.08 \\
-0.05 \\
-0.02\end{array}$ & $\begin{array}{l}a^{4} \mathrm{~F}_{21 / 2}-x^{4} \mathrm{~F}_{11 / 2}^{0} \\
a^{4} \mathrm{~F}_{31 / 2}-w^{4} \mathrm{D}_{31 / 2}^{2}\end{array}$ & $\begin{array}{l}5 \\
6\end{array}$ \\
\hline $\begin{array}{l}4114.594 \\
4115.033 \\
4116.598 \\
4119.010 \\
4122.367\end{array}$ & $\begin{array}{l}10 \mathrm{H} l \\
1 \mathrm{~h} \\
8 \mathrm{H} l \\
5 \\
20\end{array}$ & $\begin{array}{l}\text { 24296. } 90 \\
24294.31 \\
24285.08 \\
24270.85 \\
24251.09\end{array}$ & $\begin{array}{r}0.08 \\
0.03 \\
-0.01 \\
-0.08 \\
-0.01\end{array}$ & 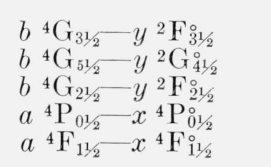 & $6,7 b$ \\
\hline $\begin{array}{l}4122.757 \\
4123.280 \\
4123.542 \\
4125.415 \\
4125.813\end{array}$ & $\begin{array}{l}12 \\
15 \\
20 \\
1 h \\
8 c\end{array}$ & $\begin{array}{l}24248.80 \\
24245.72 \\
24244.18 \\
24233.17 \\
24230.83\end{array}$ & $\begin{array}{r}-0.06 \\
-0.02 \\
0.07 \\
-0.06 \\
0.03\end{array}$ & $\begin{array}{c}a^{{ }^{4} \mathrm{~F}_{31 / 2}}-x^{4} \mathrm{~F}_{31 / 2}^{\circ} \\
a^{4} \mathrm{~F}_{21 / 2}-x{ }^{4} \mathrm{~F}_{21 / 2}^{\circ} \\
a^{4} \mathrm{P}_{21 / 2}-x{ }^{4} \mathrm{P}_{11 / 2}^{\circ} \\
a^{4} \mathrm{D}_{21 / 2}-w^{6} \mathrm{P}_{11 / 2}^{\circ} \\
a^{2} \mathrm{G}_{41 / 2}-w^{2} \mathrm{G}_{41 / 2}^{\circ}\end{array}$ & 6 \\
\hline $\begin{array}{l}\text { 4126. } 710 \\
4126.876 \\
4127.751\end{array}$ & $\begin{array}{l}1 h \\
1 \\
1\end{array}$ & $\begin{array}{l}24225.57 \\
24224.60 \\
24219.46\end{array}$ & $\begin{array}{l}0.25 \\
0.03 \\
0.06\end{array}$ & $\begin{array}{l}a^{4} \mathrm{D}_{21 / 2}-y^{6} \mathrm{D}_{31 / 2}^{\circ}{ }^{\circ} a^{4} \mathrm{~F}_{21 / 2}-w^{4} \mathrm{D}_{31 / 2}^{31} \\
a^{4} \mathrm{~F}_{11 / 2}-u^{4} \mathrm{P}_{11 / 2}^{1}\end{array}$ & \\
\hline 4130. 256 & $2 h$ & 24204. 77 & $\left\{\begin{array}{r}-0.02 \\
0.01\end{array}\right.$ & $a^{4} \mathrm{D}_{21 / 2}-y{ }^{6} \mathrm{D}_{21 / 2}^{\circ}$ & \\
\hline 4131. 111 & 150 & 24199. 76 & $\begin{array}{r}0.01 \\
-0.03\end{array}$ & $a^{4} \mathrm{H}_{61 / 2}^{21 / 2}-y^{4} \mathrm{H}_{61 / 2}^{21 / 2}$ & $6,7 b$ \\
\hline $\begin{array}{l}\text { 4131. } 449 \\
\text { 4132. } 282 \\
\text { 4134. } 619 \\
\text { 4135. } 034 \\
\text { 4137. } 266\end{array}$ & $\begin{array}{r}10 \\
6 \\
20 \\
100 \\
40\end{array}$ & $\begin{array}{l}\text { 24197. } 78 \\
24192.90 \\
24179.23 \\
24176.80 \\
24163.76\end{array}$ & $\begin{array}{r}0.13 \\
0.13 \\
0.06 \\
0.02 \\
-0.04\end{array}$ & 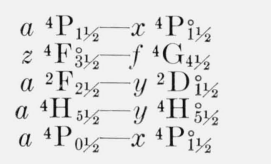 & $\begin{array}{ll}6, & 7 b \\
& 4 \\
5 \\
6, \\
7 b \\
4\end{array}$ \\
\hline $\begin{array}{l}4141.063 \\
4147.529 \\
4148.796 \\
4149.648 \\
4150.262\end{array}$ & $\begin{array}{c}100 \\
60 \\
80 \\
1 \mathrm{H} \\
1\end{array}$ & $\begin{array}{l}\text { 24141. } 60 \\
24103.97 \\
24096.61 \\
24091.66 \\
24088.10\end{array}$ & $\begin{array}{r}-0.02 \\
0.10 \\
-0.02 \\
-0.07 \\
-0.05\end{array}$ & $\begin{array}{c}a^{4} \mathrm{H}_{41 / 2}-y{ }^{4} \mathrm{H}_{41 / 2}^{\circ} \\
a^{4} \mathrm{P}_{21 / 2}-x{ }^{4} \mathrm{P}_{21 / 2}^{0} \\
a^{4} \mathrm{H}_{31 / 2}-y^{4} \mathrm{H}_{31 / 2}^{\circ} \\
a^{2} \mathrm{~F}_{31 / 2}-w^{6} \mathrm{D}_{21 / 2}^{2} \\
a^{4} \mathrm{H}_{51 / 2}-y{ }^{4} \mathrm{H}_{61 / 2}^{0}\end{array}$ & $\begin{array}{l}6,7 b \\
6,7 b \\
6,7 b\end{array}$ \\
\hline 4151. 023 & $10 h l$ & 24083.68 & $\left\{\begin{array}{r}0.28 \\
-0.22\end{array}\right.$ & $a^{4} \mathrm{H}_{41 / 2}-y^{4} \mathrm{H}_{51 / 2}^{\circ}$ & \\
\hline $\begin{array}{l}4151.663 \\
4152.556 \\
4154.218 \\
4154.631\end{array}$ & $\begin{array}{l}3 h \\
1 \\
1 \\
2\end{array}$ & $\begin{array}{l}24079.97 \\
24074.79 \\
24065.16 \\
24062.76\end{array}$ & $\begin{array}{r}-0.39 \\
-0.04 \\
0.06 \\
-0.15\end{array}$ & $\begin{array}{l}b^{4} \mathrm{G}_{41 / 2}-z^{2} \mathrm{~F}_{31 / 2}^{0} \\
a^{2} \mathrm{G}_{31 / 2}-w^{2} \mathrm{G}_{31 / 2}^{\circ} \\
b^{4} \mathrm{G}_{51 / 2}-w^{4} \mathrm{G}_{41 / 2}^{0} \\
a^{4} \mathrm{D}_{11 / 2}-w^{6} \mathrm{P}_{11 / 2}^{0}\end{array}$ & \\
\hline $\begin{array}{l}4154.726 \\
4155.525\end{array}$ & $\begin{array}{r}1 \\
40\end{array}$ & $\begin{array}{l}24062.22 \\
24057.59\end{array}$ & $\begin{array}{r}-0.03 \\
0.18\end{array}$ & $\begin{array}{c}a{ }^{4} \mathrm{H}_{31 / 2}-y{ }^{4} \mathrm{H}_{41 / 2}^{\circ} \\
a^{4} \mathrm{P}_{11 / 2}-x\end{array}$ & 4 \\
\hline 4157.019 & 60 & 24048.94 & $\left\{\begin{array}{r}-0.03 \\
0.22\end{array}\right.$ & $\begin{array}{l}b^{4} \mathrm{G}_{51 / 2}-w^{4} \mathrm{G}_{51 / 2}^{5} \\
b^{4} \mathrm{G}_{31 / 2}-y^{2} \mathrm{G}_{31 / 3}^{0} ?\end{array}$ & 6 \\
\hline $\begin{array}{l}\text { 4158. } 692 \\
4159.957\end{array}$ & $\begin{array}{l}8 \\
1\end{array}$ & $\begin{array}{l}\text { 24039. } 24 \\
\text { 24031. } 96\end{array}$ & $\begin{array}{l}-0.03 \\
-0.02\end{array}$ & $a^{2} \mathrm{P}_{01 / 2}-z^{2} \mathrm{P}_{01 / 2}^{0}$ & 6 \\
\hline $\begin{array}{l}4164.979 \\
4166.208 \\
4167.197 \\
4169.440 \\
4175.891\end{array}$ & $\begin{array}{c}10 h \\
6 \\
2 h \\
1 h \\
2 h\end{array}$ & $\begin{array}{l}24002.96 \\
23995.90 \\
23990.21 \\
23977.30 \\
23940.26\end{array}$ & $\begin{array}{r}-0.02 \\
-0.11 \\
-0.03 \\
0.00 \\
0.26\end{array}$ & $\begin{array}{l}a^{2} \mathrm{P}_{01 / 2}-z^{2} \mathrm{P}_{11 / 2}^{\circ} \\
b^{4} \mathrm{G}_{21 / 2}-y^{2} \mathrm{G}_{31 / 2}^{\circ} \\
b^{4} \mathrm{G}_{31 / 2}-z^{2} \mathrm{~F}_{21 / 2}^{\circ} \\
b^{4} \mathrm{G}_{31 / 2}-y^{2} \mathrm{G}_{41 / 2}^{\circ} \\
a^{4} \mathrm{D}_{11 / 2}-w^{6} \mathrm{P}_{21 / 2}^{\circ}\end{array}$ & 5 \\
\hline $\begin{array}{l}4176.608 \\
4179.875 \\
4182.254 \\
4184.956 \\
4189.990\end{array}$ & $\begin{array}{c}100 c \\
4 h \\
20 \mathrm{H} w \\
2 h \\
100\end{array}$ & $\begin{array}{l}23936.15 \\
23917.44 \\
23903.84 \\
23888.40 \\
23859.70\end{array}$ & $\begin{array}{l}-0.03 \\
-0.02 \\
-0.03 \\
-0.14 \\
-0.02\end{array}$ & $\begin{array}{c}a{ }^{4} \mathrm{H}_{61 / 2}-y{ }^{4} \mathrm{G}_{51 / 2}^{\circ} \\
a^{4} \mathrm{D}_{21 / 2}-y{ }^{6} \mathrm{D}_{11 / 2}^{\circ} \\
a^{2} \mathrm{H}_{51 / 2}-y^{2} \mathrm{I}_{61 / 2}^{\circ} \\
e^{8} \mathrm{~S}_{31 / 2}-63319^{\circ}{ }^{\circ}{ }^{\circ} \\
a^{4} \mathrm{H}_{51 / 2}-y{ }^{4} \mathrm{G}_{41 / 2}^{\circ}\end{array}$ & 4 \\
\hline
\end{tabular}


Table 1. Mn I-Classified lines-Continued

\begin{tabular}{|c|c|c|c|c|c|}
\hline 1 & 2 & 3 & 4 & 5 & 6 \\
\hline$\lambda$ & Int. & $\sigma$ & $\mathrm{o}-\mathrm{c}$ & Term designation & $\begin{array}{c}\text { Zeeman } \\
\text { type }\end{array}$ \\
\hline $\begin{array}{c}\AA \\
\text { Air }\end{array}$ & & $K$ & $K$ & & \\
\hline 4190.901 & $1 h$ & 23854.51 & -0.21 & $b^{4} \mathrm{G}_{41 / 2}-w^{4} \mathrm{G}_{41 / 2}^{0}$ & \\
\hline 4193. 745 & 1 & 23838. 34 & $\left\{\begin{array}{l}-0.08 \\
-0.25\end{array}\right.$ & $\begin{array}{l}a{ }^{4} \mathrm{D}_{31 / 2}-w^{6} \mathrm{P}^{0}{ }^{11 / 2} \\
b^{4} \mathrm{G}^{41}-w^{4} \mathrm{G}_{51}^{0}\end{array}$ & \\
\hline 4199. 250 & $1 h$ & 23807. 09 & -0.13 & $a^{2} \mathbf{I}_{51 / 2}^{+/ 2}-x^{4} \mathrm{H}_{41 / 2}^{0}$ & \\
\hline $\begin{array}{l}4201.778 \\
4202.612\end{array}$ & $\begin{array}{r}60 \\
1\end{array}$ & $\begin{array}{l}23792.77 \\
23788.05\end{array}$ & $\begin{array}{r}-0.02 \\
0.00\end{array}$ & $\begin{array}{l}a{ }^{4} \mathrm{H}_{41 / 2}-y^{4} \mathrm{G}_{31 / 2}^{0} \\
a^{2} \mathrm{G}_{41-2}-v{ }^{4} \mathrm{H}_{51 / 0}^{0}\end{array}$ & 4 \\
\hline 4203. 113 & 3 & 23785.21 & 0.14 & $a^{2} \mathrm{I}_{51 / 2}-x^{4} \mathrm{H}_{51 / 2}^{\circ}$ & \\
\hline 4207. 956 & $1 h l$ & 23757.84 & -0.08 & $a^{2} \mathrm{G}_{31 / 2}-w^{2} \mathrm{~F}_{31 / 2}^{0}$ & \\
\hline 4209.677 & 2 & 23748. 12 & 0.00 & $b^{4} \mathrm{G}_{31 / 2}-w^{4} \mathrm{G}_{41 / 2}^{\circ}$ & \\
\hline 4209. 864 & $1 h$ & 23747. 07 & -0.07 & $a^{4} \mathrm{D}_{11 / 2}-y{ }^{6} \mathrm{D}_{11 / 2}^{0}$ & \\
\hline 4210.519 & $2 h$ & 23743.37 & -0.01 & $b^{4} \mathrm{G}_{31 / 2}-w^{4} \mathrm{G}_{31 / 2}^{o}$ & \\
\hline 4211. 753 & 50 & 23736. 42 & -0.04 & $a^{4} \mathrm{H}_{31 / 2}-y{ }^{4} \mathrm{G}_{21 / 2}^{0}$ & 5 \\
\hline 4211. 942 & $10 \mathrm{Hl}$ & 23735. 35 & 0.01 & $a^{2} \mathrm{H}_{51 / 2}-61744_{51 / 2}^{\circ}$ & \\
\hline 4212. 443 & 8 & 23732.53 & -0.11 & $a{ }^{4} \mathrm{D}_{11 / 2}-y{ }^{6} \mathrm{D}_{01 / 2}^{0}$ & \\
\hline 4215. 842 & 1 & 23713. 40 & -0.02 & $a^{4} \mathrm{H}_{31 / 2}-y{ }^{4} \mathrm{G}_{31 / 2}^{0}$ & \\
\hline 4217. 186 & $1 h$ & 23705.84 & 0.02 & $a^{2} \mathrm{H}_{51 / 2}-y^{2} \mathrm{G}_{41 / 2}$ & \\
\hline 4218. 405 & $5 \mathrm{H} w$ & 23698. 99 & 0.10 & $a^{2} \mathrm{H}_{41 / 2}-y^{2} \mathrm{I}_{51 / 2}^{\circ}$ & \\
\hline 4220.610 & $50 \mathrm{cw}$ & 23686. 61 & -0.06 & $b{ }^{4} \mathrm{P}_{21 / 2}-y{ }^{4} \mathrm{~S}_{11 / 2}$ & 4 \\
\hline 4221.562 & $9 h$ & 23681. 27 & -0.03 & $b^{4} \mathrm{G}_{21 / 2}-w^{4} \mathrm{G}_{21 / 2}^{2}$ & \\
\hline 4224. 336 & 5 & 23665. 72 & -0.04 & $a^{2} \mathrm{H}_{41 / 2}-y^{2} \mathrm{G}_{31 / 2}^{0}$ & \\
\hline 4225. 080 & & 23661.55 & -0.04 & $b{ }^{4} \mathrm{P}_{21 / 2}-v{ }^{4} \mathrm{P}_{21 / 2}^{0}$ & \\
\hline 4225. 785 & 1 & 23657. 60 & -0.10 & $a^{2} \mathrm{G}_{31 / 2}-v^{4} \mathrm{H}_{41 / 2}^{\circ}$ & \\
\hline 4230. 144 & 10 & 23633. 22 & -0.07 & $a^{4} \mathrm{D}_{01 / 2}-y{ }^{6} \mathrm{D}_{01 / 2}^{\circ}$ & \\
\hline 4235. 154 & 400 & 23605.27 & -0.04 & $a{ }^{4} \mathrm{D}_{21 / 2}-y{ }^{4} \mathrm{P}_{11 / 2}^{\circ}$ & 4 \\
\hline 4235. 300 & 800 & 23604.45 & -0.01 & $a{ }^{4} \mathrm{D}_{31 / 2}-y{ }^{4} \mathrm{P}_{21 / 2}^{o}$ & 4 \\
\hline 4237. 104 & $2 \mathrm{H}$ & 23594. 41 & 0.07 & $a^{2} \mathrm{H}_{41 / 2}-y^{2} \mathrm{G}_{41 / 2}$ & \\
\hline 4239. 737 & 200 & 23579. 75 & -0.02 & $a^{4} \mathrm{D}_{11 / 2}-y{ }^{4} \mathrm{P}_{01 / 2}^{o}$ & 4 \\
\hline 4247. 689 & 1 & 23535. 61 & -0.03 & $b^{4} \mathrm{G}_{51 / 2}-x{ }^{4} \mathrm{H}_{41 / 2}^{0}$ & \\
\hline 4250. 722 & $1 h$ & 23518.82 & 0.09 & $b^{4} \mathrm{G}_{51 / 2}^{0 / 2}-w^{4} \mathrm{~F}_{41 / 2}^{\circ}$ & \\
\hline 4251.345 & $2 h w$ & 23515.37 & 0.09 & $b^{2} \mathrm{I}_{61 / 2}-u^{4} \mathrm{H}_{61 / 2}^{\circ}$ & \\
\hline 4257. 669 & 200 & 23480.44 & 0.02 & $a{ }^{4} \mathrm{D}_{01 / 2}-y{ }^{4} \mathrm{P}_{01 / 2}^{\circ}$ & 6 \\
\hline 4258. 369 & 7 & 23476.58 & -0.06 & $a^{2} \mathrm{H}_{51 / 2}-w^{4} \mathrm{G}_{41 / 2}^{\circ}$ & \\
\hline 4259. 349 & 10 & 23471.18 & -0.06 & $b^{4} \mathrm{G}_{51 / 2}-x{ }^{4} \mathrm{H}_{61 / 2}^{\circ}$ & 4 \\
\hline 4261. 301 & 30 & 23460.43 & -0.08 & $a^{2} \mathrm{H}_{51 / 2}-w^{4} \mathrm{G}_{51 / 2}^{1}$ & 6 \\
\hline 4265. 928 & 400 & 23434. 99 & 0.00 & $a{ }^{4} \mathrm{D}_{11 / 2}-y{ }^{4} \mathrm{P}_{11 / 2}^{0}$ & \\
\hline 4271.282 & 2 & 23405.61 & 0. 19 & $a^{2} \mathbf{F}_{31 / 2}-y^{2} \mathbf{F}_{21 / 2}^{102}$ & \\
\hline 4278.676 & 20 & 23365. 16 & 0.00 & $a^{2} \mathrm{H}_{41 / 2}^{\circ}-w^{4} \mathrm{G}_{41 / 2}^{\circ}$ & 6 \\
\hline 4279.545 & $8 \mathrm{H}$ & 23360. 42 & 0.00 & $a^{2} \mathrm{H}_{41 / 2}^{0}-w^{4} \mathrm{G}_{31 / 2}^{0}$ & \\
\hline 4281.100 & 500 & 23351. 93 & 0.00 & $a^{4} \mathrm{D}_{21 / 2}-y{ }^{4} \mathrm{P}_{21 / 2}^{\circ}$ & 6 \\
\hline 4284. 083 & 100 & 23335.67 & 0.03 & $a^{4} \mathrm{D}_{01 / 2}-y{ }^{4} \mathrm{P}_{11 / 2}^{0}$ & 5 \\
\hline 4290. 111 & 10 & 23302. 89 & -0.22 & $b^{4} \mathrm{G}_{41 / 2}-x{ }^{4} \mathrm{H}_{51 / 2}^{0}$ & 4 \\
\hline 4300. 194 & 20 & 23248. 25 & 0.00 & $b^{4} \mathrm{G}_{51 / 2}-z^{2} \mathrm{G}_{41 / 2}^{0}$ & 5 \\
\hline 4305. 670 & 10 & 23218.68 & 0.02 & $b^{4} \mathrm{G}_{31 / 2}-x^{4} \mathrm{H}_{41 / 2}^{0}$ & \\
\hline 4305. 985 & 4 & 23216.98 & $\left\{\begin{array}{r}-0.09 \\
0.13\end{array}\right.$ & $\begin{array}{l}a{ }^{2} \mathrm{H}_{51 / 2}-y{ }^{4} \mathrm{I}_{51 / 2}^{\circ} \\
a^{2} \mathrm{H}_{51-y}{ }^{4} \mathrm{I}_{61 / 2}^{\circ}\end{array}$ & \\
\hline 4308. 633 & 1 & 23202. 71 & -0.02 & $a^{2} \mathrm{H}_{51 / 2}-y^{4} \mathrm{I}_{41 / 2}^{6}$ & \\
\hline 4312.554 & 100 & 23181.62 & 0.01 & $a^{4} \mathrm{D}_{11 / 2}-y{ }^{4} \mathrm{P}_{21 / 2}^{0}$ & 5 \\
\hline 4314. 424 & $2 h$ & 23171.57 & 0.03 & $a^{4} \mathrm{~F}_{41 / 2}-y{ }^{4} \mathrm{G}_{41 / 2}^{\circ}$ & \\
\hline 4315. 235 & 8 & 23167. 22 & -0.06 & $b^{4} \mathrm{G}_{21 / 2}-x{ }^{4} \mathrm{H}_{31 / 2}^{172}$ & \\
\hline 4315.532 & $1 h$ & 23165. 62 & 0.04 & $b^{4} \mathrm{G}_{31 / 2}-w^{4} \mathrm{~F}_{31 / 2}^{0}$ & \\
\hline 4320.284 & $4 \mathrm{H}$ & 23140. 14 & 0.06 & $a^{2} \mathrm{~F}_{21 / 2}-y^{2} \mathrm{~F}_{21 / 2}^{0}$ & \\
\hline 4326. 181 & 5 & 23108.60 & -0.20 & $b^{4} \mathrm{G}_{41 / 2}-z^{2} \mathrm{G}_{31 / 2}^{0}$ & \\
\hline
\end{tabular}


TABle 1. Mn I-Classified lines-Continued

\begin{tabular}{|c|c|c|c|c|c|}
\hline 1 & 2 & 3 & 4 & 5 & 6 \\
\hline$\lambda$ & Int. & $\sigma$ & $\mathrm{o}-\mathrm{c}$ & Term designation & $\begin{array}{c}\text { Zeeman } \\
\text { type }\end{array}$ \\
\hline$\underset{\text { Air }}{\stackrel{\check{A}}{4}}$ & & $K$ & $K$ & & \\
\hline 4326. 747 & & 23105.57 & -0.02 & $a^{2} \mathrm{H}_{41 / 2}-y^{4} \mathrm{I}_{51 / 2}^{\circ}$ & \\
\hline 4327.951 & $10 \mathrm{H} w$ & 23099. 15 & 0.05 & $a^{2} \mathrm{~F}_{21 / 2}-y^{2} \mathrm{~F}_{31 / 2}^{0}$ & \\
\hline 4328.676 & & 23095. 28 & -0.04 & $a{ }^{4} \mathrm{~F}_{31 / 2}-y^{4} \mathrm{G}_{31 / 2}^{0}$ & \\
\hline 4329. 430 & 3 & 23091. 26 & $\begin{array}{l}0.01 \\
0.09\end{array}$ & $a{ }^{2} \mathrm{H}_{41 / 2}-y{ }^{4} \mathrm{I}_{41 / 2}^{0}$ & \\
\hline & & 20000.25 & & $\begin{array}{lll}31 / 2 & 0 & 13 / 2\end{array}$ & \\
\hline 4337. 414 & $30 c$ & 23048. 76 & -0.03 & $b{ }^{4} \mathrm{P}_{11 / 2}-y{ }^{4} \mathrm{~S}_{11 / 2}$ & \\
\hline 4338. 135 & $2 h$ & 23044. 92 & 0. 00 & $a^{2} \mathrm{~F}_{31 / 2}-y^{2} \mathrm{G}_{41 / 2}^{0}$ & \\
\hline 4338. 819 & $3 h$ & 23041. 29 & $\begin{array}{r}-0.09 \\
\quad 0.14\end{array}$ & $\begin{array}{l}a^{2}{ }^{2} \mathrm{~F}_{31 / 2}-z^{2} \mathrm{~F}_{31 / 2}^{1} \\
b^{4} \mathrm{P}_{11}-v{ }^{4} \mathrm{P}^{0}\end{array}$ & \\
\hline 4342.105 & 1 & 23023. 85 & $\left\{\begin{array}{r}0.14 \\
-0.04\end{array}\right.$ & $a^{2} \mathrm{G}_{41 / 2}^{11 / 2}-x^{2} \mathrm{H}_{41 / 2}^{21 / 2}$ & \\
\hline 4346. 331 & $5 h$ & 23001.47 & 0.00 & $z^{8} \mathrm{P}_{21 / 2}^{01 / 2}-e^{6} \mathrm{~S}_{21 / 2}$ & \\
\hline 4352.100 & $1 ?$ & 22970.98 & 0. 04 & $b^{4} \mathrm{G}_{21 / 2}-w^{4} \mathrm{~F}_{11 / 2}^{0}$ & \\
\hline 4356. 180 & $1 h$ & 22949.46 & -0.03 & $b^{4} \mathrm{G}_{21 / 2}-z^{2} \mathrm{G}_{31 / 2}^{0}$ & \\
\hline 4356. 613 & $\begin{array}{l}2 \\
2\end{array}$ & 22947.18 & 0. 00 & $a^{2} \mathrm{H}_{51 / 2}-x{ }^{4} \mathrm{H}_{41 / 2}$ & \\
\hline $\begin{array}{l}4359.640 \\
4359.815\end{array}$ & $8 h$ & $\begin{array}{l}22931.25 \\
22930.33\end{array}$ & $\begin{array}{r}-0.02 \\
0.06\end{array}$ & $\begin{array}{l}b^{4} \mathrm{G}_{31 / 2}-z^{2} \mathrm{G}_{41 / 2}^{\circ} \\
a^{2} \mathrm{H}_{51 / 2}-w^{4} \mathrm{~F}_{41 / 2}^{1}\end{array}$ & \\
\hline 4366. 078 & 1 & 22897.44 & 0.03 & $b^{4} \mathrm{P}_{11 / 2}-v{ }^{4} \mathrm{P}_{11 / 2}^{0}$ & \\
\hline 4368. 881 & 50 & 22882.75 & -0.03 & $a^{2} \mathrm{H}_{51 / 2}-x^{4} \mathrm{H}_{61 / 2}^{\circ}$ & \\
\hline 4370.881 & 8 & 22872.29 & 0.00 & $z^{8} \mathrm{P}_{31 / 2}^{0}-e{ }^{6} \mathrm{~S}_{21 / 2}$ & \\
\hline 4374.952 & 50 & 22851. 00 & $\left\{\begin{array}{r}-0.16 \\
0.00\end{array}\right.$ & $\begin{array}{l}a^{4} \mathrm{P}_{11 / 2}-v^{6} \mathrm{P}_{1 / 2}^{\circ} \\
a^{2} \mathrm{~F}_{21 / 2}-y^{2} \mathrm{G}_{31}^{0}\end{array}$ & $7 b$ \\
\hline 4381. 700 & 50 & 22815.80 & 0.06 & $a^{2} \mathrm{~F}_{31 / 2}^{2 / 2}-w^{4} \mathrm{G}_{41 / 2}^{\circ / 2}$ & 4 \\
\hline 4382.135 & 1 & 22813.54 & -0.01 & $a^{2} \mathrm{H}_{41 / 2}-x^{4} \mathrm{H}_{51 / 2}^{0}$ & \\
\hline 4382.620 & $40 \mathrm{H}$ & 22811. 01 & $\left\{\begin{array}{l}0.02 \\
0.01\end{array}\right.$ & $a^{4} \mathrm{P}_{21 / 2}-v{ }^{6} \mathrm{P}_{21 / 2}^{0}$ & 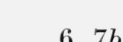 \\
\hline 4384. 410 & $2 h$ & 22801.70 & $\begin{array}{l}0.01 \\
0.07\end{array}$ & $a^{2} \mathrm{~F}_{31 / 2}-w^{4} \mathrm{G}_{21 / 2}^{31 / 2}$ & 0,10 \\
\hline 4386. 176 & $2 h$ & 22792.52 & 0.00 & $a^{2} \mathrm{~F}_{21 / 2}^{0}-z^{2} \mathrm{~F}_{21 / 2}^{\circ}$ & \\
\hline 4388. 092 & 10 & 22782.57 & -0.05 & $a^{2} \mathrm{H}_{41 / 2}-w^{4} \mathrm{~F}_{31 / 2}^{0}$ & \\
\hline 4389. 377 & $1 h$ & 22775.90 & -0.14 & $a^{2} \mathrm{~F}_{21 / 2}-z^{2} \mathrm{~F}_{31 / 2}^{\circ}$ & \\
\hline 4390.540 & $2 h$ & 22769.87 & -0.01 & $b{ }^{4} \mathrm{D}_{31 / 2}-y{ }^{4} \mathrm{D}_{31 / 2}$ & \\
\hline 4393. 413 & 8 & 22754.96 & -0.04 & $b{ }^{4} \mathrm{D}_{31 / 2}-y{ }^{4} \mathrm{D}_{21 / 2}^{0}$ & \\
\hline 4408. 083 & $5 s$ & 22679.25 & 0. 03 & $a^{2} I_{51 / 2}-z^{2} I_{51 / 2}^{0}$ & 6 \\
\hline 4410. 493 & 2 & 22666.86 & -0.04 & $b{ }^{4} \mathrm{P}_{01 / 2}-y{ }^{4} \mathrm{~S}_{11 / 2}^{0}$ & \\
\hline 4411. 874 & 10 & 22659. 77 & -0.02 & $a^{2} \mathrm{H}_{51 / 2}-z^{2} \mathrm{G}_{41 / 2}^{0}$ & 4 \\
\hline 4414.887 & 40 & 22644.30 & 0.04 & $a^{4} \mathrm{D}_{31 / 2}-z^{4} \mathrm{D}_{21 / 2}^{172}$ & 5 \\
\hline 4419. 769 & 10 & 22619. 29 & 0.05 & $a^{2} \mathrm{H}_{41 / 2}-z^{2} \mathrm{G}_{31 / 2}^{1}$ & 4 \\
\hline 4433. 720 & $1 s$ & 22548.12 & -0.19 & $a^{2} \mathrm{H}_{41 / 2}-z^{2} \mathrm{G}_{41 / 2}^{0}$ & \\
\hline 4434. 139 & 2 & 22545.98 & 0.32 & $a^{2} \mathrm{~F}_{21 / 2}-w^{4} \mathrm{G}_{31 / 2}^{0}$ & \\
\hline 4436. 061 & $6 h$ & 22536. 22 & -0.07 & $a^{2} \mathrm{~F}_{21 / 2}-w^{4} \mathrm{G}_{21 / 2}^{0}$ & \\
\hline 4436. 358 & 80 & 22534. 70 & 0.01 & $a^{4} \mathrm{D}_{21 / 2}-z^{4} \mathrm{D}_{11 / 2}$ & 5 \\
\hline 4439.873 & 0 & 22516.87 & -0.14 & $a^{2} \mathrm{G}_{41 / 2}-z^{2} \mathrm{H}_{41 / 2}^{0}$ & \\
\hline 4451.575 & 100 & 22457.66 & 0.06 & $a^{4} \mathrm{D}_{31 / 2}-z^{4} \mathrm{D}_{31 / 2}^{\circ}$ & $6,7 b$ \\
\hline 4452.525 & $7 s$ & 22452.88 & 0.01 & $a^{2} \mathrm{I}_{61 / 2}-z^{2} \mathrm{I}_{61 / 2}^{\circ}$ & \\
\hline 4453. 013 & 50 & 22450.42 & 0.01 & $a^{4} \mathrm{D}_{11 / 2}-z^{4} \mathrm{D}_{01 / 2}^{\circ}$ & 5 \\
\hline 4455. 019 & 25 & 22440.32 & 0.00 & $z^{6} \mathrm{P}_{11 / 2}^{0}-e^{6} \mathrm{D}_{01 / 2}$ & \\
\hline 4455. 320 & 25 & 22438.80 & -0.03 & $z^{6} \mathrm{P}_{11 / 2}^{0}-e^{6} \mathrm{D}_{11 / 2}$ & \\
\hline 4455. 820 & 25 & 22436. 29 & 0. 00 & $z^{6} \mathrm{P}_{11 / 2}^{0}-e^{6} \mathrm{D}_{21 / 2}$ & \\
\hline 4457. 041 & 20 & 22430.14 & 0.04 & $z^{6} \mathrm{P}_{21 / 2}^{0}-e^{6} \mathrm{D}_{11 / 2}$ & \\
\hline 4457.553 & 20 & 22427.56 & 0.00 & $z^{6} \mathrm{P}_{21 / 2}^{\circ}-e^{6} \mathrm{D}_{21 / 2}$ & \\
\hline 4458. 263 & 25 & 22423. 99 & -0.02 & $z^{6} \mathrm{P}_{21 / 2}^{0}-e^{6} \mathrm{D}_{31 / 2}$ & \\
\hline 4460. 376 & 20 & 22413. 37 & 0.01 & $z^{6} \mathrm{P}_{31 / 2}^{0}-e^{6} \mathrm{D}_{21 / 2}$ & \\
\hline 4461. 089 & 30 & 22409.84 & 0.03 & $z^{6} \mathrm{P}_{31 / 2}^{o}-e^{6} \mathrm{D}_{31 / 2}^{0 / 2}$ & \\
\hline 4462. 033 & 150 & 22405.05 & 0.02 & $z^{6} \mathrm{P}_{31 / 2}^{0}-e^{6} \mathrm{D}_{41 / 2}$ & \\
\hline
\end{tabular}


TABle 1. Mn I-Classified lines-Continued

\begin{tabular}{|c|c|c|c|c|c|}
\hline 1 & 2 & 3 & 4 & 5 & 6 \\
\hline$\lambda$ & Int. & $\sigma$ & $\mathrm{o}-\mathrm{c}$ & Term designation & $\begin{array}{l}\text { Zeeman } \\
\text { type }\end{array}$ \\
\hline$\underset{\mathrm{Air}}{\AA}$ & & $K$ & $K$ & & \\
\hline 4464.679 & 80 & 22391. 76 & 0.03 & $a^{4} \mathrm{D}_{21 / 2}-z^{4} \mathrm{D}_{21 / 2}^{\circ}$ & $6,7 b$ \\
\hline 4470.142 & 60 & 22364.40 & 0.03 & $a^{4} \mathrm{D}_{11 / 2}-z^{4} \mathrm{D}_{11 / 2}$ & $6,7 b$ \\
\hline 4472. 793 & 100 & 22351. 14 & 0.08 & $a^{4} \mathrm{D}_{01 / 2}-z{ }^{4} \mathrm{D}_{01 / 2}$ & 6,0 \\
\hline $\begin{array}{l}4473.52 \\
4479.399\end{array}$ & $\begin{array}{c}2 \\
10 s\end{array}$ & $\begin{array}{l}22347.2 \\
22318.18\end{array}$ & $\begin{array}{c}0.1 \\
-0.01\end{array}$ & $\begin{array}{l}a^{4} \mathrm{~F}_{11 / 2}-y^{4} \mathrm{~S}_{11 / 2}^{0} \\
a^{2} \mathrm{G}_{31 / 2}-z^{2} \mathrm{H}_{41 / 2}^{0}\end{array}$ & 5 \\
\hline 4489. 23 & 2 & 22269.3 & -0.1 & $a^{2} \mathrm{~F}_{31,2}-w^{4} \mathrm{~F}_{4 ! / 2}^{0}$ & \\
\hline 4490.078 & 30 & 22265.10 & 0. 08 & $a{ }^{4} \mathrm{D}_{01 / 2}-z{ }^{4} \mathrm{D}_{11 / 2}^{0}$ & 5 \\
\hline 4491. 652 & $10 s$ & 22257.30 & $\left\{\begin{array}{l}0.00 \\
0.00\end{array}\right.$ & $\begin{array}{l}a^{2} \mathrm{G}_{41 / 2}-z^{2} \mathrm{H}_{51 / 2}^{\circ} \\
a^{2} \mathrm{G}_{41-}-x^{2} \mathrm{~F}_{31 / 2}^{0}\end{array}$ & 5 \\
\hline 4496. 638 & $8 s$ & 22232.62 & -0.04 & $b^{4} \mathrm{G}_{51 / 2}-x^{4} \mathrm{G}_{51 / 2}^{012}$ & 6 \\
\hline 4498. 897 & 20 & 22221.45 & 0.04 & $a^{4} \mathrm{D}_{11 / 2}-z^{4} \mathrm{D}_{21 / 2}$ & 5 \\
\hline 4502. 223 & 30 & 22205. 04 & -0.03 & $a^{4} \mathrm{D}_{21 / 2}-z^{4} \mathrm{D}_{31 / 2}^{\circ}$ & 5 \\
\hline 4503. 868 & 15 & 22196. 93 & 0.05 & $b^{4} \mathrm{G}_{51 / 2}^{27 / 2}-z^{2} \mathrm{I}_{61 / 2}^{0}$ & 4 \\
\hline 4512.659 & 2 & 22153.69 & 0.00 & $b^{4} \mathrm{G}_{41 / 2}-x^{4} \mathrm{G}_{31 / 2}^{\circ}$ & \\
\hline 4520.016 & 1 & 22117.63 & 0.02 & $a^{2} \mathrm{G}_{31 / 2}-y^{2} \mathrm{H}_{41 / 2}^{\circ / 2}$ & \\
\hline 4523. 399 & $3 s$ & 22101.09 & -0.23 & $b{ }^{4} \mathrm{G}_{41 / 2}-x{ }^{4} \mathrm{G}_{41 / 2}$ & $6,7 b$ \\
\hline 4529.804 & $3 s$ & 22069.84 & 0.02 & $a^{2} \mathrm{~F}_{31 / 2}-z^{2} \mathrm{G}_{31 / 2}^{0}$ & 6 \\
\hline 4534.481 & $2.5 s$ & 22047.08 & -0.01 & $b^{4} \mathrm{G}_{31 / 2}^{072}-x^{4} \mathrm{G}_{31 / 2}^{0,2}$ & $6,7 b$ \\
\hline 4538.475 & 2 & 22027.68 & -0.09 & $b^{4} \mathrm{G}_{21 / 2}-x^{4} \mathrm{G}_{21 / 2}^{\circ}$ & \\
\hline 4541.26 & 2 & 22014. 2 & -0.1 & $a^{2} \mathrm{P}_{11 / 2}-w^{4} \mathrm{D}_{21 / 2}^{2}$ & \\
\hline 4544. 423 & $5 s$ & 21998.85 & -0.04 & $a^{2} \mathrm{~F}_{31 / 2}-z^{2} \mathrm{G}_{41 / 2}^{0}$ & 4 \\
\hline 4546.32 & 12 & 21994.5 & $\left\{\begin{array}{r}0.1 \\
-0.2\end{array}\right.$ & $\begin{array}{l}b^{4} \mathrm{G}_{21 / 2}-x^{4} \mathrm{G}_{31 / 2}^{0} \\
b^{4} \mathrm{G}_{31 / 2}-x^{4} \mathrm{G}^{011 / 2}\end{array}$ & \\
\hline 4561.13 & 2 & 21918. 2 & 0. 2 & $z^{6} \mathrm{P}_{21 / 2}^{0}-e^{8} \mathrm{D}_{11 / 2}$ & \\
\hline 4563. 62 & 2 & 21906.4 & 0.3 & $z^{6} \mathrm{P}_{31 / 2}^{0}-e^{8} \mathrm{D}_{31 / 3}$ & \\
\hline 4564.0 & 2 & 21904.5 & -0.3 & $z^{6} \mathrm{P}_{31 / 2}^{o}-e^{8} \mathrm{D}_{21 / 2}^{0}$ & \\
\hline 4565.77 & 10 & 21896. 0 & 0. 0 & $y^{6} \mathrm{P}_{21 / 2}^{0}-f^{4} \mathrm{D}_{11 / 2}$ & \\
\hline 4578.077 & 1 & 21837.13 & -0.07 & $b^{4} \mathrm{G}_{51 / 2}-x^{4} \mathrm{~F}_{41 / 2}^{\circ}$ & \\
\hline 4581.854 & 2 & 21819.13 & -0.05 & $a^{2} \mathrm{H}_{51 / 2}-z^{2} \mathrm{I}_{51 / 2}^{\circ}$ & \\
\hline 4584.918 & 1 & 21804.56 & 0.08 & $a^{2} \mathrm{~F}_{21 / 2}-z^{2} \mathrm{G}_{31 / 2}^{0}$ & \\
\hline 4586.114 & $(30 h)$ & 21798.04 & -0.38 & $a^{2} \mathrm{P}_{11 / 2}-u{ }^{4} \mathrm{P}_{11 / 2}^{1}$ & \\
\hline 4598.940 & 1 & 21738.08 & 0.12 & $b^{4} \mathrm{G}_{21 / 2}-v^{4} \mathrm{D}_{11 / 2}^{1} ?$ & \\
\hline 4605. 365 & 20 & 21707.75 & 0.05 & $a^{2} \mathrm{H}_{41 / 2}-z^{2} \mathrm{I}_{51 / 2}^{\circ}$ & 5 \\
\hline 4606.38 & 1 & 21702.9 & 0.0 & $b^{4} \mathrm{D}_{11 / 2}-u^{6} \mathrm{P}_{21 / 2}^{2}$ & \\
\hline 4607.61 & $7 \mathrm{~s}$ & 21697. 2 & 0.0 & $a^{4} \mathrm{D}_{31 / 2}-x^{6} \mathrm{P}_{31 / 2}^{\circ}$ & \\
\hline 4614. 12 & 1 & 21666.6 & 0.0 & $b^{4} \mathrm{P}_{21 / 2}-v^{6} \mathrm{~F}_{31 / 2}^{0} ?$ & \\
\hline 4615.65 & 8 & 21659.4 & -0.2 & $b^{4} \mathrm{G}_{41 / 2}^{2 / 2}-x{ }^{4} \mathrm{~F}_{31 / 2}^{\circ}$ & \\
\hline 4620. 21 & $5 h$ & 21638.0 & 0.0 & $a^{2} \mathrm{P}_{01 / 2}-w^{4} \mathrm{D}_{11 / 2}^{\circ}$ & \\
\hline 4622.74 & 8 & 21626.2 & 0.0 & $b^{4} \mathrm{G}_{21 / 2}-x{ }^{4} \mathrm{~F}_{11 / 2}$ & \\
\hline 4623.33 & 8 & 21623.4 & -0.1 & $b^{4} \mathrm{G}_{31 / 2}-x{ }^{4} \mathrm{~F}_{21 / 2}^{0}$ & \\
\hline 4624.220 & 8 & 21619. 22 & 0. 29 & $y^{6} \mathrm{P}_{31 / 2}^{0}-e^{8} \mathrm{P}_{41 / 2}$ & \\
\hline 4626.544 & 25 & 21608.37 & -0.05 & $a^{2} \mathrm{H}_{51 / 2}-z^{2} \mathrm{I}_{6^{1 / 2}}^{0}$ & 4 \\
\hline 4626.861 & 2 & 21606.89 & -0.02 & $a^{4} \mathrm{D}_{21 / 2}-x^{6} \mathrm{P}_{21 / 2}^{\circ}$ & \\
\hline 4642.803 & $5 s$ & 21532.70 & -0.02 & $a^{2} \mathrm{H}_{41 / 2}^{21 / 2}-x^{4} \mathrm{G}_{51 / 2}^{202}$ & \\
\hline 4658.42 & 12 & 21460.5 & 0.1 & $b^{4} \mathrm{P}_{11 / 2}-55923_{21 / 2}^{2}$ & \\
\hline 4661.93 & 2 & 21444. 4 & -0.3 & $a^{4} \mathrm{D}_{21 / 2}-x{ }^{6} \mathrm{P}_{31 / 2}^{0}$ & \\
\hline 4663. 638 & 2 & 21436.51 & -0.08 & $a{ }^{4} \mathrm{D}_{11 / 2}-x{ }^{6} \mathrm{P}_{21 / 2}^{0}$ & \\
\hline 4671.688 & $10 s$ & 21399.57 & -0.05 & $a{ }^{4} \mathrm{D}_{31 / 2}-z^{4} \mathrm{~F}_{21 / 2}^{\circ}$ & 5 \\
\hline 4697.52 & 2 & 21281.9 & 0.1 & $b^{4} \mathrm{P}_{21 / 2}^{\circ}-x^{4} \mathrm{D}_{31 / 2}^{\circ}$ & \\
\hline 4701. 150 & $8 s$ & 21265.46 & -0.07 & $a^{4} \mathrm{D}_{21 / 2}-z^{4} \mathrm{~F}_{11 / 2}$ & \\
\hline $\begin{array}{l}4709.710 \\
4712.02\end{array}$ & 40 & 21226.81 & 0. 03 & $a^{4} \mathrm{D}_{31 / 2}-z^{4} \mathrm{~F}_{31 / 2}^{\circ}$ & 6 \\
\hline & & 21216.5 & 0.0 & $a{ }^{2} \mathrm{P}_{01 / 2}-u{ }^{4} \mathrm{P}_{01 / 2}^{\circ}$ & \\
\hline
\end{tabular}


Table 1. Mn I-Classified lines-Continued

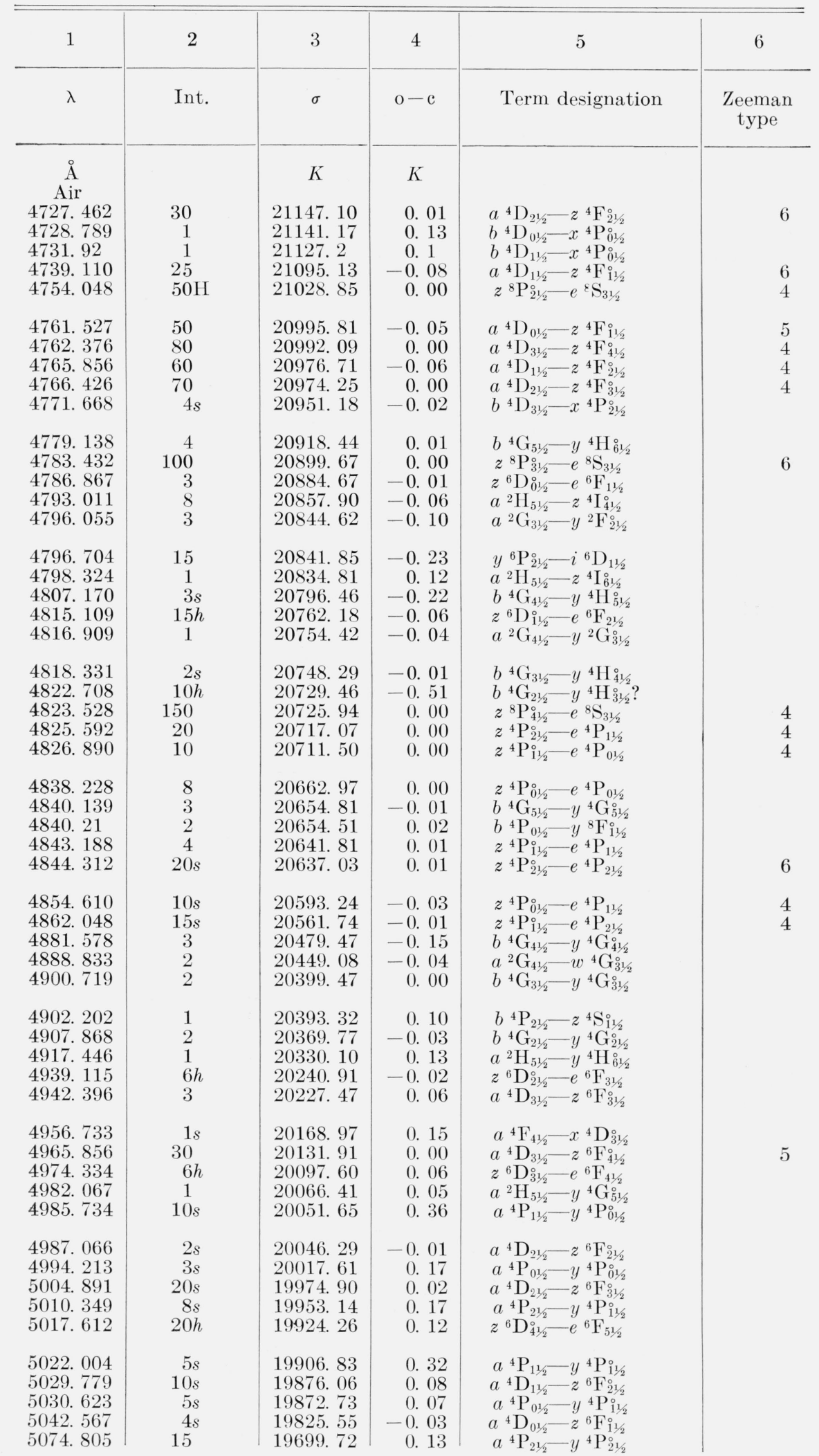


Table 1. Mn I-Classified lines - Continued

\begin{tabular}{|c|c|c|c|c|c|}
\hline 1 & 2 & 3 & 4 & 5 & 6 \\
\hline$\lambda$ & Int. & $\sigma$ & $o-c$ & Term designation & $\begin{array}{c}\text { Zeeman } \\
\text { type }\end{array}$ \\
\hline $\begin{array}{c}\text { A } \\
\text { Air } \\
5086.884 \\
5091.018 \\
5117.935 \\
5142.919 \\
5149.155\end{array}$ & $\begin{array}{l}10 s \\
1 s \\
20 s \\
15 s \\
50\end{array}$ & $\begin{array}{c}K \\
\text { 19652. } 94 \\
\text { 19636. } 98 \\
\text { 19533. } 70 \\
19438.81 \\
19415.27\end{array}$ & $\begin{array}{c}K \\
-0.19 \\
-0.03 \\
0.01 \\
0.02 \\
0.02\end{array}$ & $\begin{array}{c}a^{4} \mathrm{P}_{11 / 2}-y^{4} \mathrm{P}_{21 / 2}^{\circ} \\
a^{2} \mathrm{G}_{41 / 2}-z^{2} \mathrm{G}_{41 / 2}^{\circ} \\
a^{4} \mathrm{G}_{21 / 2}-z^{4} \mathrm{~F}_{11 / 2}^{0} \\
z^{6} \mathrm{~F}_{11 / 2}^{0}-e{ }^{6} \mathrm{~F}_{11 / 2} \\
a^{4} \mathrm{G}_{21 / 2}-z^{4} \mathrm{~F}_{21 / 2}^{\circ 1}\end{array}$ & $\mathrm{P}-\mathrm{B}$ \\
\hline $\begin{array}{l}5150.937 \\
5177.077 \\
5180.298 \\
5190.02 \\
5190.69\end{array}$ & $\begin{array}{l}30 s \\
2 \\
2 s \\
6 h \\
6 h\end{array}$ & $\begin{array}{l}19408.55 \\
19310.55 \\
19298.55 \\
19262.40 \\
19259.91\end{array}$ & $\begin{array}{r}0.00 \\
0.24 \\
-0.05 \\
-0.64 \\
-0.01\end{array}$ & 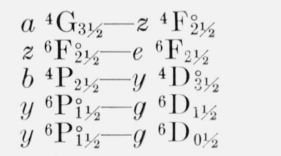 & \\
\hline $\begin{array}{l}5196.603 \\
5197.229 \\
5199.51 \\
5200.18 \\
5201.440\end{array}$ & $\begin{array}{l}30 s \\
10 \\
3 \\
5 \\
2 \mathrm{H}\end{array}$ & $\begin{array}{l}19238.00 \\
19235.68 \\
19227.24 \\
19224.77 \\
19220.11\end{array}$ & $\begin{array}{r}-0.02 \\
-0.03 \\
0.07 \\
-0.04 \\
-0.13\end{array}$ & 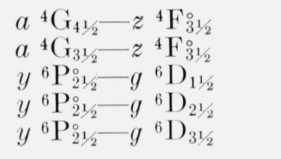 & \\
\hline $\begin{array}{l}\text { 5213. } 34 \\
5215.434 \\
5249.371 \\
5255.330 \\
5260.771\end{array}$ & $\begin{array}{l}8 \\
5 \mathrm{H} \\
2 \\
30 \\
8 s\end{array}$ & $\begin{array}{l}19176.24 \\
19168.54 \\
19044.60 \\
19023.02 \\
19003.34\end{array}$ & $\begin{array}{r}0.12 \\
-0.05 \\
-0.01 \\
0.00 \\
0.01\end{array}$ & $\begin{array}{l}y{ }^{6} \mathrm{P}_{31 / 2}^{\circ}-g^{6} \mathrm{D}_{31 / 2} \\
y{ }^{6} \mathrm{P}_{31 / 2}^{\circ}-g^{6} \mathrm{D}_{41 / 2} \\
b^{4} \mathrm{P}_{21 / 2}-x \\
{ }^{6} \mathrm{D}_{31 / 2}^{0} \\
a^{4} \mathrm{G}_{51 / 2}-z^{4} \mathrm{~F}_{41 / 2}^{\circ} \\
a^{4} \mathrm{G}_{41 / 2}-z^{4} \mathrm{~F}_{41 / 2}^{0}\end{array}$ & 4 \\
\hline $\begin{array}{l}5274.206 \\
5277.54 \\
5284.196 \\
5289.495 \\
5292.869\end{array}$ & $\begin{array}{l}2 \\
8 \mathrm{H} \\
6 \mathrm{H} \\
5 h \\
1\end{array}$ & $\begin{array}{l}18954.94 \\
18942.96 \\
18919.10 \\
18900.15 \\
18888.10\end{array}$ & $\left\{\begin{array}{r}-0.02 \\
0.29 \\
0.01 \\
-0.30 \\
-0.40 \\
0.02\end{array}\right.$ & $\begin{array}{l}z^{6} \mathrm{~F}_{31 / 2}^{\circ}-e e^{4} \mathrm{G}_{41 / 2} \\
z^{4} \mathrm{~F}_{41 / 2}^{\circ}-e^{4} \mathrm{~F}_{41 / 2} \\
z^{6} \mathrm{~F}_{21 / 2}^{0}-e e^{6} \mathrm{G}_{21 / 2}^{\circ} ? \\
z^{6} \mathrm{~F}_{01 / 2}^{\circ}-e^{6} \mathrm{G}_{11 / 2} \\
z^{4} \mathrm{~F}_{31 / 2}^{\circ}-e e^{4} \mathrm{~F}_{31 / 2} \\
a^{4} \mathrm{P}_{01 / 2}-z^{4} \mathrm{D}_{01 / 2}^{\circ}\end{array}$ & \\
\hline $\begin{array}{l}5297.920 \\
5298.84 \\
5308.92 \\
5317.082 \\
5324.317\end{array}$ & $\begin{array}{l}3 h d \\
7 s \\
15 h \\
8 s \\
20 h\end{array}$ & $\begin{array}{l}18870.10 \\
18866.82 \\
18831.00 \\
18802.09 \\
18776.54\end{array}$ & $\begin{array}{r}-0.04 \\
0.04 \\
0.02 \\
0.05 \\
-0.01\end{array}$ & $\begin{array}{l}z^{6} \mathrm{~F}_{11 / 2}^{\circ}-e^{6} \mathrm{G}_{21 / 2} \\
z^{6} \mathrm{~F}_{11 / 2}^{\circ}-e^{4} \mathrm{G}_{51 / 2}^{\circ} \\
z^{6} \mathrm{~F}_{21 / 2}^{\circ}-e^{6} \mathrm{G}_{31 / 2}^{\circ} \\
a^{4} \mathrm{P}_{01 / 2}-z^{4} \mathrm{~J}_{11 / 2}^{\circ} \\
z^{6} \mathrm{~F}_{31 / 2}^{0}-e^{6} \mathrm{G}_{41 / 2}^{0}\end{array}$ & \\
\hline $\begin{array}{l}5325.999 \\
5334.872 \\
5341.065 \\
5344.438 \\
5348.078\end{array}$ & $\begin{array}{c}5 h \\
5 s \\
250 \\
50 \mathrm{H} \\
20 s\end{array}$ & $\begin{array}{l}18770.61 \\
18739.39 \\
18717.66 \\
18705.85 \\
18693.12\end{array}$ & $\begin{array}{r}0.03 \\
0.00 \\
-0.02 \\
-0.02 \\
0.19\end{array}$ & $\begin{array}{c}z^{6} \mathrm{~F}_{31 / 2}^{\circ}-e^{6} \mathrm{~F}_{31 / 2} \\
a^{4} \mathrm{P}_{21 / 2}-z^{4} \mathrm{D}_{21 / 2}^{\circ} \\
a^{6} \mathrm{D}_{41 / 2}-y \\
{ }^{6} \mathrm{P}_{31 / 2}^{0} \\
z^{6} \mathrm{~F}_{41 / 2}^{\circ}-e{ }^{6} \mathrm{G}_{51 / 2}^{\circ} \\
a^{4} \mathrm{P}_{11 / 2}^{\circ}-z^{4} \mathrm{D}_{21 / 2}^{\circ}\end{array}$ & 4 \\
\hline $\begin{array}{l}5349.878 \\
5361.631 \\
5364.488 \\
5374.39 \\
5375.306\end{array}$ & $\begin{array}{l}80 \mathrm{H} \\
1 \\
5 \\
1 h \\
2\end{array}$ & $\begin{array}{l}18686.83 \\
18645.86 \\
18635.94 \\
18601.60 \\
18598.42\end{array}$ & $\begin{array}{r}-0.03 \\
0.02 \\
-0.03 \\
0.00 \\
0.00\end{array}$ & $\begin{array}{c}z^{6} \mathrm{~F}_{51 / 2}^{\circ}-e^{6} \mathrm{G}_{61 / 2} \\
b^{4} \mathrm{P}_{11 / 2}-y{ }^{4} \mathrm{D}_{21 / 2}^{\circ} \\
a^{4} \mathrm{D}_{31 / 2}-z^{6} \mathrm{D}_{31 / 2}^{\circ} \\
z^{6} \mathrm{~F}_{41 / 2}^{\circ}-e^{6} \mathrm{~F}_{41 / 2}^{\circ} \\
z^{4} \mathrm{H}_{61 / 2}^{\circ}-f^{4} \mathrm{G}_{51 / 2}^{\circ} ?\end{array}$ & \\
\hline $\begin{array}{l}5377.634 \\
5388.538 \\
5394.677 \\
5399.506 \\
5402.608\end{array}$ & $\begin{array}{c}100 \\
15 s \\
30 \\
30 \\
1\end{array}$ & $\begin{array}{l}18590.38 \\
18552.80 \\
18531.65 \\
18515.08 \\
18504.45\end{array}$ & $\begin{array}{r}0.02 \\
0.07 \\
0.01 \\
-0.01 \\
-0.08\end{array}$ & $\begin{array}{c}z^{4} \mathrm{P}_{21 / 2}-e^{4} \mathrm{~S}_{11 / 2} \\
a^{4} \mathrm{P}_{21 / 2}-z^{4} \mathrm{D}_{31 / 2}^{0} \\
a^{6} \mathrm{~S}_{21 / 2}-z z^{8} \mathrm{P}_{31 / 2}^{0} \\
z^{4} \mathrm{P}_{11 / 2}-e^{4} \mathrm{~S}_{11 / 2}^{0} \\
a^{4} \mathrm{D}_{21 / 2}-z{ }^{6} \mathrm{D}_{21 / 2}\end{array}$ & $\begin{array}{l}4 \\
6\end{array}$ \\
\hline $\begin{array}{l}5406.014 \\
5407.432 \\
5413.696 \\
5420.368 \\
5426.192\end{array}$ & $\begin{array}{c}5 \\
50 c \\
30 \\
100 c \\
2\end{array}$ & $\begin{array}{l}18492.79 \\
18487.97 \\
18466.55 \\
18443.82 \\
18424.03\end{array}$ & $\begin{array}{r}-0.02 \\
0.00 \\
-0.01 \\
-0.03 \\
-0.02\end{array}$ & $\begin{array}{l}a{ }^{4} \mathrm{D}_{31 / 2}-z{ }^{6} \mathrm{D}_{41 / 2}^{\circ} \\
a{ }^{6} \mathrm{D}_{31 / 2}-y \\
{ }^{6} \mathrm{P}_{31 / 2}^{\circ} \\
z^{4} \mathrm{P}_{01 / 2}^{\circ}-e^{4} \mathrm{~S}_{11 / 2}^{11 / 2} \\
a^{6} \mathrm{D}_{31 / 2}-y{ }^{6} \mathrm{P}_{21 / 2}^{0} \\
a^{4} \mathrm{D}_{11 / 2}-z{ }^{6} \mathrm{D}_{11 / 2}^{0}\end{array}$ & $\begin{array}{l}6 \\
4 \\
4\end{array}$ \\
\hline $\begin{array}{l}5427.184 \\
5432.555 \\
5433.422 \\
5439.290 \\
5444.094\end{array}$ & $\begin{array}{c}1 h \\
60 \\
100 w c \\
0 \\
1 h\end{array}$ & $\begin{array}{l}18420.66 \\
18402.45 \\
18399.50 \\
18379.66 \\
18363.44\end{array}$ & $\begin{array}{r}0.16 \\
-0.01 \\
0.11 \\
-0.03 \\
0.03\end{array}$ & $\begin{array}{l}b^{4} \mathrm{P}_{11 / 2}-x^{6} \mathrm{D}_{11 / 2}^{\circ}, 21 / 2 \\
a^{6} \mathrm{~S}_{21 / 2}-z^{8} \mathrm{P}_{21 / 2}^{\circ} \\
z^{6} \mathrm{~F}_{51 / 2}^{\circ}-e^{6} \mathrm{~F}_{51 / 2} \\
a^{4} \mathrm{D}_{01 / 2}-z^{6} \mathrm{D}_{01 / 2}^{\circ} \\
a^{4} \mathrm{G}_{21 / 2}-z^{6} \mathrm{~F}_{11 / 2}^{\circ}\end{array}$ & \\
\hline
\end{tabular}


TABLE 1. Mn I-Classified lines-Continued

\begin{tabular}{|c|c|c|c|c|c|}
\hline 1 & 2 & 3 & 4 & 5 & 6 \\
\hline$\lambda$ & Int. & $\sigma$ & $\mathrm{o}-\mathrm{c}$ & Term designation & $\begin{array}{c}\text { Zeeman } \\
\text { type }\end{array}$ \\
\hline $\begin{array}{c}\text { A } \\
\text { Air } \\
5445.99 \\
5447.576 \\
5457.468 \\
5460.645 \\
5470.640\end{array}$ & $\begin{array}{r}1 h \\
1 h \\
30 \\
1 \\
100\end{array}$ & $\begin{array}{c}K \\
18357.04 \\
18351.70 \\
18318.44 \\
18307.78 \\
18274.33\end{array}$ & $\begin{array}{r}K \\
0.10 \\
-0.02 \\
-0.01 \\
0.02 \\
0.00\end{array}$ & 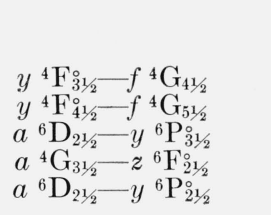 & 6 \\
\hline $\begin{array}{l}\text { 5481. } 345 \\
5495.913 \\
5497.374 \\
5504.224 \\
5505.877\end{array}$ & $\begin{array}{l}100 \\
1 \\
10 h \\
15 \\
50\end{array}$ & $\begin{array}{l}18238.48 \\
18190.30 \\
18185.47 \\
18162.83 \\
18157.38\end{array}$ & $\begin{array}{r}0.02 \\
0.02 \\
0.08 \\
-0.01 \\
0.01\end{array}$ & $\begin{array}{l}a{ }^{6} \mathrm{D}_{21 / 2}-y{ }^{6} \mathrm{P}_{11 / 2}^{\circ} \\
z^{4} \mathrm{~F}_{41 / 2}^{\circ}-e^{4} \mathrm{G}_{41 / 2}^{\circ} \\
a^{4} \mathrm{~F}_{41 / 2}-y{ }^{4} \mathrm{D}_{31 / 2}^{\circ} \\
a^{4} \mathrm{G}_{51 / 2}-z{ }^{6} \mathrm{~F}_{41 / 2}^{\circ} \\
a^{6} \mathrm{D}_{11 / 2}-y{ }^{6} \mathrm{P}_{21 / 2}^{\circ}\end{array}$ & \\
\hline $\begin{array}{l}5510.190 \\
5516.777 \\
5520.496 \\
5533.156 \\
5536.471\end{array}$ & $\begin{array}{r}1 \\
100 \\
10 h \\
5 s \\
8 h\end{array}$ & $\begin{array}{l}18143.16 \\
18121.50 \\
18109.29 \\
18067.86 \\
18057.04\end{array}$ & $\begin{array}{r}0.01 \\
0.00 \\
-0.03 \\
0.02 \\
-0.04\end{array}$ & $\begin{array}{c}a^{4} \mathrm{G}_{41 / 2}-z^{6} \mathrm{~F}_{41 / 2}^{\circ} \\
a^{6} \mathrm{D}_{11 / 2}^{1}-y{ }^{6} \mathrm{P}_{11 / 2}^{\circ} \\
z^{4} \mathrm{~F}_{31 / 2}^{\circ}-e^{4} \mathrm{G}_{31 / 2}^{\circ} \\
a^{4} \mathrm{~F}_{31 / 2}-y{ }^{4} \mathrm{D}_{21 / 2}^{\circ} \\
z^{4} \mathrm{~F}_{21 / 2}^{\circ}-e^{4} \mathrm{G}_{21 / 2}^{\circ}\end{array}$ & 6 \\
\hline $\begin{array}{l}\text { 5537. } 749 \\
5551.978 \\
5555.782 \\
5567.764 \\
5573.010\end{array}$ & $\begin{array}{c}100 \\
80 \mathrm{w} \\
3 s \\
50 \\
30\end{array}$ & $\begin{array}{l}18052.88 \\
18006.60 \\
17994.29 \\
17955.55 \\
17938.67\end{array}$ & $\begin{array}{r}0.05 \\
0.00 \\
0.06 \\
-0.04 \\
0.03\end{array}$ & $\begin{array}{l}a{ }^{6} \mathrm{D}_{01 / 2}-y{ }^{6} \mathrm{P}_{11 / 2}^{\circ} \\
z^{4} \mathrm{~F}_{41 / 2}^{\circ}-e^{4} \mathrm{G}_{51 / 2}^{\circ} \\
a^{4} \mathrm{~F}_{21 / 2}-y{ }^{4} \mathrm{D}_{21 / 2}^{\circ} \\
z^{4} \mathrm{~F}_{31 / 2}^{\circ}-e{ }^{4} \mathrm{G}_{41 / 2}^{\circ} \\
z^{4} \mathrm{~F}_{11 / 2}^{\circ}-e^{4} \mathrm{G}_{21 / 2}^{\circ}\end{array}$ & 4 \\
\hline $\begin{array}{l}5573.690 \\
5575.242 \\
5602.046 \\
5603.08 \\
5607.355\end{array}$ & $\begin{array}{r}40 \\
1 \\
5 \\
1 \\
1\end{array}$ & $\begin{array}{l}17936.48 \\
17931.46 \\
17845.68 \\
17842.38 \\
17828.78\end{array}$ & $\begin{array}{r}0.00 \\
0.06 \\
-0.01 \\
-0.12 \\
0.05\end{array}$ & $\begin{array}{l}z^{4} \mathrm{~F}_{21 / 2}^{\circ}-e^{4} \mathrm{G}_{31 / 2} \\
a^{4} \mathrm{~F}_{41 / 2}-x{ }^{6} \mathrm{D}_{31 / 2}^{\circ} \\
z^{4} \mathrm{~F}_{41 / 2}^{\circ}-e^{6} \mathrm{G}_{51 / 2}^{\circ} \\
a^{4} \mathrm{~F}_{31 / 2}-x{ }^{6} \mathrm{D}_{21 / 2}^{\circ} \\
a^{4} \mathrm{~F}_{31 / 2}-x{ }^{6} \mathrm{D}_{31 / 2}^{\circ}\end{array}$ & \\
\hline $\begin{array}{l}5626.247 \\
5642.394 \\
5658.395 \\
5687.133 \\
5718.236\end{array}$ & $\begin{array}{l}3 \\
1 \\
1 \\
1 \\
2 h\end{array}$ & $\begin{array}{l}17768.93 \\
17718.07 \\
17667.97 \\
17578.68 \\
17483.07\end{array}$ & $\begin{array}{r}0.04 \\
0.02 \\
0.05 \\
-0.05 \\
0.00\end{array}$ & $\begin{array}{l}a^{4} \mathrm{~F}_{21 / 2}-x^{6} \mathrm{D}_{11 / 2}^{\circ}, 21 / 2 \\
a^{4} \mathrm{~F}_{11 / 2}-x^{6} \mathrm{D}_{01 / 2}^{\circ} \\
b^{4} \mathrm{G}_{31 / 2}-w^{4} \mathrm{P}_{21 / 2}^{\circ} \\
b^{4} \mathrm{G}_{21 / 2}-\mathrm{W}^{4} \mathrm{P}_{11 / 2}^{\circ} \\
z^{4} \mathrm{D}_{21 / 2}^{0}-e^{4} \mathrm{~F}_{31 / 2}^{\circ}\end{array}$ & \\
\hline $\begin{array}{l}\text { 5720. } 233 \\
5738.286 \\
5780.173 \\
5782.307 \\
5816.840\end{array}$ & $\begin{array}{l}2 h \\
30 \\
25 \\
1 h \\
20\end{array}$ & $\begin{array}{l}17476.97 \\
17422.00 \\
17295.74 \\
17289.34 \\
17186.71\end{array}$ & $\begin{array}{r}-0.19 \\
-0.05 \\
-0.01 \\
-0.26 \\
0.00\end{array}$ & $\begin{array}{l}z^{4} \mathrm{D}_{31 / 2}^{\circ}-e^{4} \mathrm{~F}_{41 / 2} \\
a^{4} \mathrm{H}_{61 / 2}-z^{4} \mathrm{G}_{51 / 2}^{\circ} \\
a^{4} \mathrm{H}_{51 / 2}-z^{4} \mathrm{G}_{11 / 2}^{\circ} \\
a^{2} \mathrm{G}_{31 / 2}-y{ }^{4} \mathrm{H}_{31 / 2}^{\circ} \\
a^{4} \mathrm{H}_{41 / 2}-z^{4} \mathrm{G}_{31 / 2}^{\circ}\end{array}$ & \\
\hline $\begin{array}{l}5822.55 \\
5835.372 \\
5848.950 \\
5868.18 \\
5879.18\end{array}$ & $\begin{array}{r}3 \\
5 \\
15 \\
2 \\
2\end{array}$ & $\begin{array}{l}17169.86 \\
17132.12 \\
17092.36 \\
17036.35 \\
17004.47\end{array}$ & $\begin{array}{r}-0.09 \\
0.03 \\
0.00 \\
0.09 \\
-0.09\end{array}$ & $\begin{array}{l}z^{4} \mathrm{G}_{41 / 2}^{0}-f^{4} \mathrm{G}_{41 / 2} \\
z^{4} \mathrm{G}_{51 / 2}^{0}-f^{4} \mathrm{G}_{51 / 2} \\
a^{4} \mathrm{H}_{31 / 2}-z^{4} \mathrm{G}_{21 / 2}^{0} \\
x^{6} \mathrm{P}_{31 / 2}^{0}-e^{6} \mathrm{~F}_{41 / 2} \\
y^{6} \mathrm{P}_{21 / 2}^{0}-f^{6} \mathrm{D}_{31 / 2}\end{array}$ & \\
\hline $\begin{array}{l}\text { 5973. } 867 \\
6013.484 \\
6014.385 \\
6016.697 \\
6021.787\end{array}$ & $\begin{array}{c}2 \\
100 \\
20 s \\
150 \\
200\end{array}$ & $\begin{array}{l}16734.95 \\
16624.70 \\
16622.21 \\
16615.82 \\
16601.78\end{array}$ & $\begin{array}{r}0.05 \\
0.09 \\
-0.02 \\
-0.06 \\
0.10\end{array}$ & $\begin{array}{l}b{ }^{4} \mathrm{D}_{21 / 2}-y{ }^{4} \mathrm{P}_{11 / 2}^{\circ} \\
z^{6} \mathrm{P}_{11 / 2}^{\circ}-e^{6} \mathrm{~S}_{21 / 2} \\
a^{4} \mathrm{~F}_{41 / 2}-z^{4} \mathrm{G}_{51 / 2}^{\circ} \\
z^{6} \mathrm{P}_{21 / 2}^{0}-e{ }^{6} \mathrm{~S}_{21 / 2} \\
z^{6} \mathrm{P}_{31 / 2}^{0}-e \mathrm{~S}_{21 / 2}^{0}\end{array}$ & $\begin{array}{l}6 \\
4\end{array}$ \\
\hline $\begin{array}{l}\text { 6041. } 730 \\
6057.120 \\
6062.885 \\
6090.080 \\
6095-656\end{array}$ & $\begin{array}{r}8 s \\
15 s \\
3 s \\
10 s \\
2 s\end{array}$ & $\begin{array}{l}16546.98 \\
16504.94 \\
16489.24 \\
16415.61 \\
16400.60\end{array}$ & $\begin{array}{r}0.06 \\
0.04 \\
0.00 \\
-0.02 \\
-0.05\end{array}$ & $\begin{array}{l}b{ }^{4} \mathrm{D}_{31 / 2}-y{ }^{4} \mathrm{P}_{21 / 2}^{\circ} \\
a^{4} \mathrm{~F}_{31 / 2}-z^{4} \mathrm{G}_{41 / 2}^{\circ} \\
a^{4} \mathrm{~F}_{31 / 2}-z^{4} \mathrm{G}_{31 / 2}^{\circ} \\
a^{4} \mathrm{~F}_{21 / 2}-z^{4} \mathrm{G}_{31 / 2}^{\circ} \\
a^{4} \mathrm{~F}_{21 / 2}-z^{4} \mathrm{G}_{21 / 2}^{0}\end{array}$ & \\
\hline $\begin{array}{l}\text { 6114. } 324 \\
6177.5 \\
6265.627 \\
6315.064 \\
6344.133\end{array}$ & $\begin{array}{l}8 s \\
2 \\
10 s \\
10 s \\
30\end{array}$ & $\begin{array}{l}16350.52 \\
16183.31 \\
15955.69 \\
15830.78 \\
15758.23\end{array}$ & $\begin{array}{r}-0.06 \\
-0.08 \\
-0.03 \\
-0.01 \\
0.04\end{array}$ & $\begin{array}{l}a^{4} \mathrm{~F}_{11 / 2}-z^{4} \mathrm{G}_{21 / 2}^{\circ} \\
e^{6} \mathrm{D}_{31 / 2}-w^{4} \mathrm{H}_{31 / 2}^{0} \\
a^{4} \mathrm{H}_{61 / 2}-z^{4} \mathrm{H}_{61 / 2}^{0} \\
a^{4} \mathrm{H}_{51 / 2}-z^{4} \mathrm{H}_{51 / 2}^{\circ} \\
b^{4} \mathrm{D}_{01 / 2}-z^{4} \mathrm{D}_{01 / 2}^{5}\end{array}$ & \\
\hline
\end{tabular}


Table 1. Mn I-Classified lines-Continued

\begin{tabular}{|c|c|c|c|c|c|}
\hline 1 & 2 & 3 & 4 & 5 & 6 \\
\hline$\lambda$ & Int. & $\sigma$ & $\mathrm{o}-\mathrm{c}$ & Term designation & $\begin{array}{l}\text { Zeeman } \\
\text { type }\end{array}$ \\
\hline $\begin{array}{c}\text { A } \\
\text { Air } \\
6349.795 \\
6356.095 \\
6378.969 \\
6382.195 \\
6384.686\end{array}$ & $\begin{array}{l}15 \\
8 s \\
20 \\
25 \\
30\end{array}$ & $\begin{array}{c}K \\
15744.19 \\
15728.59 \\
15672.19 \\
15664.27 \\
15658.16\end{array}$ & $\begin{array}{r}K \\
-0.03 \\
-0.10 \\
0.04 \\
-0.01 \\
-0.02\end{array}$ & $\begin{array}{l}b{ }^{4} \mathrm{D}_{11 / 2}-z{ }^{4} \mathrm{D}_{01 / 2}^{\circ} \\
a{ }^{4} \mathrm{H}_{41 / 2}-z \\
b^{4} \mathrm{H}_{41 / 2}^{\circ} \mathrm{D}_{01 / 2}-z^{4} \mathrm{D}_{11 / 2}^{\circ} \\
b^{4} \mathrm{D}_{011 / 2} \mathrm{D}_{21 / 2}-z^{4} \mathrm{D}_{11 / 2}^{\circ} \\
b^{4} \mathrm{D}_{11 / 2}-z{ }^{4} \mathrm{D}_{11 / 2}^{\circ}\end{array}$ & \\
\hline $\begin{array}{l}\text { 6391. } 248 \\
6413.947 \\
6440.973 \\
6443.511 \\
6483.061\end{array}$ & $\begin{array}{l}7 s \\
2 \\
50 \\
20 \\
2 s\end{array}$ & $\begin{array}{l}15642.08 \\
15586.72 \\
15521.32 \\
15515.21 \\
15420.56\end{array}$ & $\begin{array}{r}-0.11 \\
0.00 \\
0.00 \\
-0.01 \\
-0.02\end{array}$ & $\begin{array}{c}a{ }^{4} \mathrm{H}_{31 / 2}-z z^{4} \mathrm{H}_{31 / 2}^{\circ} \\
b{ }^{4} \mathrm{D}_{31 / 2}-z{ }^{4} \mathrm{D}_{21 / 2}^{\circ} \\
b^{4} \mathrm{D}_{21 / 2}-z{ }^{4} \mathrm{D}_{21 / 2}^{21} \\
b^{4} \mathrm{D}_{11 / 2}-z^{4} \mathrm{D}_{21 / 2}^{\circ} \\
a^{4} \mathrm{~F}_{41 / 2}-y^{4} \mathrm{~F}_{31 / 2}^{\circ}\end{array}$ & \\
\hline $\begin{array}{l}6590.61 \\
6491.697 \\
6519.381 \\
6520.548 \\
6526.528\end{array}$ & $\begin{array}{l}3 \\
50 \\
20 \\
2 h \\
8\end{array}$ & $\begin{array}{l}15402.62 \\
15400.04 \\
15334.65 \\
15331.90 \\
15317.86\end{array}$ & $\begin{array}{r}0.02 \\
-0.02 \\
-0.01 \\
0.04 \\
-0.05\end{array}$ & 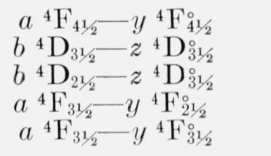 & \\
\hline $\begin{array}{l}\text { 6534. } 103 \\
6547.715 \\
6552.035 \\
6558.016 \\
6569.267\end{array}$ & $\begin{array}{l}6 \mathrm{HH} \\
2 h \\
5 \\
2 h \\
5\end{array}$ & $\begin{array}{l}15300.10 \\
15268.29 \\
15258.23 \\
15244.31 \\
15218.20\end{array}$ & $\begin{array}{r}0.17 \\
0.00 \\
-0.02 \\
0.01 \\
-0.02\end{array}$ & 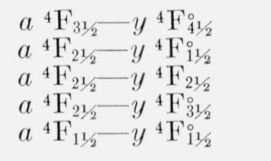 & \\
\hline $\begin{array}{l}6570.807 \\
6572.936 \\
6573.589 \\
6586.316 \\
6605.549\end{array}$ & $\begin{array}{l}7 \mathrm{HH} \\
2 \mathrm{~s} \\
1 \mathrm{~h} \\
8 \mathrm{HH} \\
10 \mathrm{HH}\end{array}$ & $\begin{array}{l}\text { 15214. } 64 \\
15209.71 \\
15208.20 \\
15178.81 \\
15134.62\end{array}$ & $\begin{array}{r}-0.06 \\
-0.29 \\
0.02 \\
-0.02 \\
-0.09\end{array}$ & 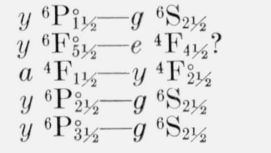 & \\
\hline $\begin{array}{l}\text { 6827. } 161 \\
6833.793 \\
6833.936 \\
6863.08 \\
6867.18\end{array}$ & $\begin{array}{l}3 h \\
5 h \\
10 h \\
4 \mathrm{H} \\
3 \mathrm{HH}\end{array}$ & $\begin{array}{l}14643.34 \\
14629.13 \\
14628.83 \\
14566.71 \\
14558.01\end{array}$ & $\begin{array}{r}0.08 \\
-0.18 \\
-0.23 \\
-0.02 \\
-0.14\end{array}$ & $\begin{array}{c}z^{6} \mathrm{P}_{21 / 2}^{\circ}-e^{8} \mathrm{~S}_{31 / 2} \\
z^{6} \mathrm{D}_{31 / 2}^{\circ}-e^{4} \mathrm{D}_{21 / 2} \\
z^{6} \mathrm{P}_{31 / 2}-e e^{8} \mathrm{~S}_{31 / 2} \\
z^{6} \mathrm{D}_{41 / 2}^{\circ}-i{ }^{6} \mathrm{D}_{31 / 2} \\
z^{6} \mathrm{D}_{31 / 2}^{0}-i{ }^{6} \mathrm{D}_{21 / 2}\end{array}$ & \\
\hline $\begin{array}{l}6880.64 \\
6887.73 \\
6890.764 \\
6924.690 \\
6931.165\end{array}$ & $\begin{array}{l}5 \mathrm{H} \\
15 \mathrm{~s} \\
1 \mathrm{HHH} \\
3 \mathrm{H} \\
10 \mathrm{H}\end{array}$ & $\begin{array}{l}14529.53 \\
14514.57 \\
14508.18 \\
14437.10 \\
14423.62\end{array}$ & $\begin{array}{r}0.09 \\
0.37 \\
-0.04 \\
0.04 \\
0.05\end{array}$ & $\begin{array}{l}z^{6} \mathrm{D}_{31 / 2}^{\circ}-e^{4} \mathrm{D}_{31 / 2} \\
z^{6} \mathrm{D}_{21 / 2}^{\circ}-i{ }^{6} \mathrm{D}^{1}{ }_{1 / 2} \\
z^{6} \mathrm{D}_{21 / 2}-e^{4} \mathrm{D}_{21 / 2} \\
z^{6} \mathrm{D}_{21 / 2}^{\circ}-i{ }^{6} \mathrm{D}_{21 / 2} \\
z^{6} \mathrm{D}_{31 / 2}^{0}-i{ }^{6} \mathrm{D}_{31 / 2}\end{array}$ & \\
\hline $\begin{array}{l}\text { 6933. } 654 \\
6938.486\end{array}$ & $\begin{array}{l}3 \mathrm{HH} \\
4 \mathrm{H}\end{array}$ & $\begin{array}{l}\text { 14418. } 44 \\
14408.40\end{array}$ & $\begin{array}{l}0.06 \\
0.05\end{array}$ & $\begin{array}{l}z^{6} \mathrm{D}_{11 / 2}^{\circ}-e^{4} \mathrm{D}_{21 / 2} \\
z^{6} \mathrm{D}_{21 / 2}^{\circ}-e^{4} \mathrm{D}_{31 / 2}\end{array}$ & \\
\hline 6941.045 & $2 \mathrm{H}$ & 14403. 09 & $\left\{\begin{array}{l}0.10 \\
0.02\end{array}\right.$ & $\begin{array}{l}b^{4} \mathrm{D}_{01 / 2}-z^{4} \mathrm{~F}_{11 / 2}^{1} \\
z^{6} \mathrm{D}_{01 / 2}^{0}-e^{4} \mathrm{D}_{11 / 2}^{0}\end{array}$ & \\
\hline $\begin{array}{l}6942.538 \\
6947.828\end{array}$ & $\begin{array}{c}20 \mathrm{H} \\
1\end{array}$ & $\begin{array}{l}\text { 14399. } 99 \\
14389.02\end{array}$ & $\begin{array}{l}0.02 \\
0.00\end{array}$ & $\begin{array}{l}z^{6} \mathrm{D}_{41 / 2}^{\circ}-i^{6} \mathrm{D}_{41 / 2} \\
b^{4} \mathrm{D}_{11 / 2}-z^{4} \mathrm{~F}_{11 / 2}^{\circ}\end{array}$ & \\
\hline $\begin{array}{l}6957.328 \\
6960.942 \\
6968.062 \\
6970.567 \\
6989.914\end{array}$ & $\begin{array}{l}1 \mathrm{~h} \\
1 \mathrm{~h} \\
3 \mathrm{HH} \\
1 \\
15 \mathrm{~h}\end{array}$ & $\begin{array}{l}14369.37 \\
14361.91 \\
14347.24 \\
14342.09 \\
14302.39\end{array}$ & $\begin{array}{r}0.00 \\
0.05 \\
0.02 \\
0.01 \\
-0.09\end{array}$ & $\begin{array}{c}z^{6} \mathrm{D}_{01 / 2}^{\circ}-i{ }^{6} \mathrm{D}_{11 / 2} \\
y^{6} \mathrm{~F}_{21 / 2}^{\circ}-e^{4} \mathrm{G}_{31 / 2} \\
z^{6} \mathrm{D}_{11 / 2}-i{ }^{6} \mathrm{D}_{21 / 2} \\
b^{4} \mathrm{D}_{31 / 2}-z^{4} \mathrm{~F}_{21 / 2}^{\circ} \\
z^{6} \mathrm{D}_{21 / 2}^{0}-i{ }^{6} \mathrm{D}_{31 / 2}\end{array}$ & \\
\hline $\begin{array}{l}7002.50 \\
7005.500 \\
7012.256 \\
7013.15 \\
7014.09\end{array}$ & $\begin{array}{c}4 s \\
2 s \\
10 \mathrm{H} \\
2 \mathrm{H} \\
1 \mathrm{H}\end{array}$ & $\begin{array}{l}14276.68 \\
14270.57 \\
14256.82 \\
14255.00 \\
14253.09\end{array}$ & $\begin{array}{r}0.00 \\
-0.01 \\
0.01 \\
0.01 \\
0.04\end{array}$ & $\begin{array}{l}b{ }^{4} \mathrm{D}_{21 / 2}-z^{4} \mathrm{~F}_{21 / 2}^{\circ} \\
b^{4} \mathrm{D}_{11 / 2}-z^{4} \mathrm{~F}_{21 / 2}^{\circ} \\
z^{6} \mathrm{D}_{31 / 2}^{\circ}-i{ }^{6} \mathrm{D}_{411 / 2}^{\circ} \\
y^{6} \mathrm{~F}_{01 / 2}^{\circ}-e^{6} \mathrm{G}_{11 / 2} \\
y^{6} \mathrm{~F}_{31 / 2}^{\circ}-e^{4} \mathrm{G}_{41 / 2}^{\circ}\end{array}$ & \\
\hline
\end{tabular}


TABLE 1. Mn I-Classified lines-Continued

\begin{tabular}{|c|c|c|c|c|c|}
\hline 1 & 2 & 3 & 4 & 5 & 6 \\
\hline$\lambda$ & Int. & $\sigma$ & $\mathrm{o}-\mathrm{c}$ & Term designation & $\begin{array}{c}\text { Zeeman } \\
\text { type }\end{array}$ \\
\hline $\begin{array}{c}\text { A } \\
\text { Air }\end{array}$ & & $K$ & $K$ & & \\
\hline 7033.555 & $1 \mathrm{HHH}$ & 14213. 65 & 0.04 & $y{ }^{6} \mathrm{~F}_{11 / 2}^{\circ}-e{ }^{6} \mathrm{G}_{21 / 2}$ & \\
\hline 7055.583 & & 14169. 27 & 0. 03 & $b^{4} \mathrm{D}_{31 / 2}-z^{4} \mathrm{~F}_{31 / 2}^{0}$ & \\
\hline 7062.42 & $1 \mathrm{HH}$ & 14155. 55 & -0.02 & $y^{6} \mathrm{~F}_{21 / 2}^{\circ}-e^{6} \mathrm{G}_{31 / 2}^{0}$ & \\
\hline $\begin{array}{l}7069.834 \\
7076.553\end{array}$ & $20 s$ & $\begin{array}{l}\text { 14140. } 71 \\
14127.28\end{array}$ & $\begin{array}{r}0.02 \\
-0.07\end{array}$ & $b^{4} \mathrm{G}_{51 / 2}-z^{4} \mathrm{G}_{51 / 2}^{0}$ & \\
\hline . & & 19126.20 & & $\begin{array}{llll}9 & 1 & 41 / 2 & 0\end{array}$ & \\
\hline 7077.145 & 1 & 14126. 10 & 0.07 & $b^{4} \mathrm{G}_{51 / 2}-z^{4} \mathrm{G}_{41 / 2}^{0}$ & \\
\hline 7088. 288 & $3 s$ & 14103. 89 & 0.05 & $b^{4} \mathrm{D}_{21 / 2}-z^{4} \mathrm{~F}_{31 / 2}^{\circ}$ & \\
\hline 7103. 010 & $2 \mathrm{HH}$ & 14074.66 & 0. 02 & $y^{6} \mathrm{~F}_{31 / 2}-e^{6} \mathrm{G}_{41 / 2}$ & \\
\hline $\begin{array}{l}\text { 7151. } 267 \\
7158.039\end{array}$ & $\begin{array}{l}5 \mathrm{HH} \\
2 \mathrm{HH}\end{array}$ & $\begin{array}{l}\text { 13979. } 69 \\
13966.46\end{array}$ & $\begin{array}{l}0.03 \\
0.02\end{array}$ & $\begin{array}{l}y^{6} \mathrm{~F}_{51 / 2}^{\circ}-e^{6} \mathrm{G}_{61 / 2} \\
y^{6} \mathrm{~F}_{41 / 2}^{\circ}-e^{6} \mathrm{G}_{51 / 2}\end{array}$ & \\
\hline 7174.420 & $4 s$ & 13934.57 & 0.02 & $b^{4} \mathrm{D}_{31 / 2}-z^{4} \mathrm{~F}_{41 / 2}^{\circ}$ & \\
\hline 7176.705 & $2 h$ & 13930. 14 & -0.17 & $b^{4} \mathrm{G}_{41 / 2}^{31 / 2}-z^{4} \mathrm{G}_{51 / 2}^{41 / 2}$ & \\
\hline 7184.29 & $15 \mathrm{~s}$ & 13915. 43 & -0.22 & $b^{4} \mathrm{G}_{41 / 2}-z^{4} \mathrm{G}_{41 / 2}^{\circ}$ & \\
\hline 7192.363 & $1 h$ & 13899.81 & -0.18 & $b^{4} \mathrm{G}_{41 / 2}-z^{4} \mathrm{G}_{31 / 2}^{0}$ & \\
\hline 7211.93 & 2 & 13862. 17 & 0.00 & $y^{6} \mathrm{~F}_{41 / 2}^{1}-e^{6} \mathrm{~F}_{41 / 2}$ & \\
\hline 7239. 600 & $3 s$ & 13809. 02 & -0.03 & $b^{4} \mathrm{G}_{31 / 2}-z^{4} \mathrm{G}_{41 / 2}^{0}$ & \\
\hline 7247.821 & $10 \mathrm{~s}$ & 13793. 45 & 0. 06 & $b^{4} \mathrm{G}_{31 / 2}-z^{4} \mathrm{G}_{31 / 2}^{+12}$ & \\
\hline 7275.690 & 1 & 13740.62 & -0.06 & $b^{4} \mathrm{G}_{21 / 2}-z^{4} \mathrm{G}_{31 / 2}^{0}$ & \\
\hline 7283. 80 & 100 & 13725.32 & -0.05 & $y^{6} \mathrm{P}_{11 / 2}^{0}-f^{6} \mathrm{~S}_{21 / 2}$ & \\
\hline 7287.10 & $1 \mathrm{HH}$ & 13719. 10 & -0.05 & $a^{6} \mathrm{D}_{31 / 2}-z{ }^{4} \mathrm{P}_{21 / 2}^{2}$ & \\
\hline 7301. 38 & $1 \mathrm{HH}$ & 13692. 27 & 0. 08 & $y^{6} \mathrm{~F}_{51 / 2}^{\circ}-e^{6} \mathrm{~F}_{51 / 2}$ & \\
\hline 7302.89 & 300 & 13689. 44 & -0.06 & $y^{6} \mathrm{P}_{21 / 2}-f^{6} \mathrm{~S}_{21 / 2}$ & \\
\hline 7322. 205 & 1 & 13653. 34 & 0. 06 & $a^{4} \mathrm{H}_{51 / 2}-y{ }^{6} \mathrm{D}_{41 / 2}^{\circ}$ & \\
\hline $\begin{array}{l}7326.500 \\
7376.85\end{array}$ & $\begin{array}{r}400 \\
3\end{array}$ & $\begin{array}{l}13645.33 \\
13552.19\end{array}$ & $\begin{array}{l}-0.05 \\
-0.04\end{array}$ & $\begin{array}{l}y^{6} \mathrm{P}_{31 / 2}^{o}-f^{6} \mathrm{~S}_{21 / 2} \\
a^{2} \mathrm{H}_{51 / 2}-z^{4} \mathrm{G}_{51 / 2}^{0}\end{array}$ & \\
\hline 7378.98 & 1 & 13548. 28 & -0.05 & $z^{6} \mathrm{D}_{11 / 2}^{\circ}-h^{6} \mathrm{D}_{11 / 2} ?$ & \\
\hline 7443.50 & 1 & 13430.85 & 0. 23 & $a^{4} \mathrm{H}_{41 / 2}-y^{6} \mathrm{D}_{31 / 2}^{18}$ & \\
\hline 7446.16 & 1 & 13426.05 & -0.04 & $a^{2} \mathrm{H}_{41 / 2}-z^{4} \mathrm{G}_{41 / 2}^{0}$ & \\
\hline 7667.89 & $1 \mathrm{H}$ & 13037.81 & -0.06 & $z^{6} \mathrm{~F}_{31 / 2}^{\circ}-e^{4} \mathrm{D}_{21 / 2}^{1 / 2}$ & \\
\hline 7670.42 & $2 \mathrm{H}$ & 13033.51 & 0. 01 & $z^{6} \mathrm{~F}_{41 / 2}^{0}-e{ }^{4} \mathrm{D}_{31 / 2}$ & \\
\hline 7677.46 & $2 \mathrm{H} l$ & 13021.56 & -0.05 & $z^{6} \mathrm{~F}_{11 / 2}^{0}-i{ }^{6} \mathrm{D}_{01 / 2}$ & \\
\hline 7680.22 & $50 \mathrm{H}$ & 13016.88 & 0. 02 & $z^{4} \mathrm{~F}_{41 / 2}^{0}-f^{4} \mathrm{D}_{31 / 2}^{0}$ & \\
\hline 7706.52 & $1 \mathrm{HHH}$ & 12972. 46 & 0. 03 & $z^{6} \mathrm{~F}_{21 / 2}^{0}-i{ }^{6} \mathrm{D}_{11 / 2}$ & \\
\hline 7709.98 & ЗНHН & 12966.64 & $\left\{\begin{array}{r}0.19 \\
-0.07\end{array}\right.$ & $\begin{array}{l}z^{6} \mathrm{~F}_{21 / 2}^{2}-e^{4} \mathrm{D}_{21 / 2} \\
z^{6} \mathrm{~F}^{\circ}-i\end{array}$ & \\
\hline 7712.42 & $10 \mathrm{H}$ & 12962.54 & 0.02 & $z^{4} \mathrm{~F}_{31 / 2}^{31 / 2}-f^{4} \mathrm{D}_{21 / 2}^{2 / 2}$ & \\
\hline 7727.07 & $1 \mathrm{H}$ & 12937.96 & -0.04 & $z^{6} \mathrm{~F}_{31 / 2}^{\circ}-e^{4} \mathrm{D}_{31 / 2}$ & \\
\hline 7733. 24 & $10 \mathrm{H}$ & 12927.64 & 0.01 & $z^{6} \mathrm{~F}_{41 / 2}^{\circ}-i{ }^{6} \mathrm{D}_{31 / 2}^{0}$ & \\
\hline 7734. 43 & $5 \mathrm{H}$ & 12925.66 & 0.05 & $z^{4} \mathrm{~F}_{21 / 2}^{\circ}-f^{4} \mathrm{D}_{11 / 2}^{\circ}$ & \\
\hline 7735.77 & $1 \mathrm{H}$ & 12923. 41 & -0.07 & $z^{6} \mathrm{~F}_{11 / 2}-i{ }^{6} \mathrm{D}_{11 / 2}$ & \\
\hline 7737.16 & $3 s$ & 12921.09 & 0. 03 & $b^{4} \mathrm{G}_{51 / 2}-y{ }^{4} \mathrm{~F}_{41 / 2}^{\circ}$ & \\
\hline 7752.67 & $1 \mathrm{HH}$ & 12895. 24 & $\left\{\begin{array}{l}-0.03 \\
-0.05\end{array}\right.$ & $z^{6} \mathrm{~F}_{01 / 2}^{\circ}-i{ }^{6} \mathrm{D}_{11 / 2}$ & \\
\hline 7755.15 & $3 \mathrm{HHH}$ & 12891. 11 & $\begin{array}{r}-0.01 \\
0.01\end{array}$ & $z^{4} \mathrm{~F}_{11 / 2}^{21 / 2}-f^{4} \mathrm{D}_{01 / 2}$ & \\
\hline 7764.72 & $50 \mathrm{H}$ & 12875. 23 & 0. 01 & $z^{6} \mathrm{~F}_{51 / 2}^{\circ}-i{ }^{6} \mathrm{D}_{41 / 2}$ & \\
\hline 7782. 2 & $1 h$ & 12846.3 & 0. 0 & $z^{6} \mathrm{~F}_{11 / 2}-i{ }^{6} \mathrm{D}_{21 / 2}$ & \\
\hline 7790.82 & & 12832.10 & -0.03 & $z^{6} \mathrm{~F}_{31 / 2}^{1}-i{ }^{6} \mathrm{D}_{31 / 2}$ & \\
\hline 7806.00 & $1 \mathrm{HHH}$ & 12807.14 & -0.03 & $z^{4} \mathrm{~F}_{11 / 2}^{\circ}-f^{4} \mathrm{D}_{11 / 2}$ & \\
\hline 7816. 61 & $3 \mathrm{H}$ & 12789.76 & 0. 08 & $z^{4} \mathrm{~F}_{21 / 2}^{0}-f^{4} \mathrm{D}_{21 / 2}$ & \\
\hline 7821.25 & $3 \mathrm{H}$ & 12782.17 & 0.00 & $z^{4} \mathrm{~F}_{31 / 2}-f{ }^{4} \mathrm{D}_{31 / 2}$ & \\
\hline 7834.34 & $10 h$ & 12760.81 & $\left\{\begin{array}{r}0.10 \\
-0.06\end{array}\right.$ & $\begin{array}{l}z^{6} \mathrm{~F}_{21 / 2}^{0}-i{ }^{6} \mathrm{D}_{31 / 2} \\
z^{6} \mathrm{~F}_{41 / 2}^{0}-i{ }^{6} \mathrm{D}_{41 / 2}\end{array}$ & \\
\hline 7854.24 & 3 & 12728.48 & -0.18 & $b^{4} \mathrm{G}_{41 / 2}^{4 / 2}-y^{4} \mathrm{~F}_{31 / 2}^{\circ}$ & \\
\hline
\end{tabular}


Table 1. Mn I-Classified lines-Continued

\begin{tabular}{|c|c|c|c|c|c|}
\hline 1 & 2 & 3 & 4 & 5 & 6 \\
\hline$\lambda$ & Int. & $\sigma$ & $\mathrm{o}-\mathrm{c}$ & Term designation & $\begin{array}{c}\text { Zeeman } \\
\text { type }\end{array}$ \\
\hline $\begin{array}{c}\AA \\
\text { Air } \\
\text { 7865. } 36 \\
7889.61 \\
7911.68 \\
7920.44 \\
7928.45\end{array}$ & $\begin{array}{l}2 \\
1 h \\
2 \\
2 \\
4\end{array}$ & $\begin{array}{c}K \\
12710.48 \\
12671.42 \\
12636.07 \\
12622.10 \\
12609.34\end{array}$ & $\begin{array}{c}K \\
-0.20 \\
0.18 \\
0.06 \\
0.04 \\
0.01\end{array}$ & $\begin{array}{l}b{ }^{4} \mathrm{G}_{41 / 2}-y{ }^{4} \mathrm{~F}_{41 / 2}^{0} \\
z^{4} \mathrm{~F}_{11 / 2}-f{ }^{4} \mathrm{D}_{21 / 2} \\
b^{4} \mathrm{G}_{31 / 2}-y{ }^{4} \mathrm{~F}_{21 / 2}^{\circ} \\
b^{4} \mathrm{G}_{31 / 2}-y{ }^{4} \mathrm{~F}_{31 / 2}^{\circ} \\
z^{4} \mathrm{~F}_{21 / 2}^{0}-f^{4} \mathrm{D}_{31 / 2}\end{array}$ & \\
\hline $\begin{array}{l}\text { 7938. } 52 \\
8043.37 \\
8194.06 \\
8210.16 \\
8212.43\end{array}$ & $\begin{array}{l}2 \\
8 \\
1 \\
2 \\
40 h l\end{array}$ & $\begin{array}{l}12593.35 \\
12429.18 \\
12200.61 \\
12176.69 \\
12173.32\end{array}$ & $\begin{array}{r}0.01 \\
0.00 \\
-0.15 \\
0.04 \\
0.00\end{array}$ & $\begin{array}{l}b{ }^{4} \mathrm{G}_{21 / 2}-y{ }^{4} \mathrm{~F}_{11 / 2}^{0} \\
a{ }^{4} \mathrm{D}_{31 / 2}-y{ }^{6} \mathrm{P}_{21 / 2}^{\circ} \\
z^{4} \mathrm{H}_{61 / 2}-e \\
{ }^{4} \mathrm{G}_{51 / 2} \\
a^{4} \mathrm{D}_{21 / 2}-y{ }^{6} \mathrm{P}_{21 / 2}^{0} \\
z^{4} \mathrm{~F}_{41 / 2}^{0}-e{ }^{4} \mathrm{D}_{31 / 2}\end{array}$ & \\
\hline $\begin{array}{l}8234.43 \\
8251.64 \\
8284.48 \\
8304.42 \\
8353.79\end{array}$ & $\begin{array}{l}1 \\
5 h \\
4 h \\
5 \mathrm{H} l \\
2 \mathrm{H}\end{array}$ & $\begin{array}{l}12140.80 \\
12115.48 \\
12067.45 \\
12038.48 \\
11967.33\end{array}$ & $\begin{array}{r}0.02 \\
0.04 \\
0.00 \\
-0.02 \\
-0.01\end{array}$ & $\begin{array}{l}a{ }^{4} \mathrm{D}_{21 / 2}-y{ }^{6} \mathrm{P}_{11 / 2} \\
b{ }^{4} \mathrm{P}_{21 / 2}-z \\
{ }^{4} \mathrm{D}_{21 / 2}^{\circ} \\
z^{4} \mathrm{~F}_{41 / 2}-i \\
z^{6} \mathrm{D}_{31 / 2} \\
z^{4} \mathrm{~F}_{31 / 2}^{o}-e{ }^{4} \mathrm{D}_{21 / 2} \\
z^{4} \mathrm{~F}_{31 / 2}^{\circ}-i{ }^{6} \mathrm{D}_{21 / 2}\end{array}$ & \\
\hline $\begin{array}{l}8373.93 \\
8380.77 \\
8395.87 \\
8409.88 \\
8421.12\end{array}$ & $\begin{array}{l}4 h \\
40 \\
10 \mathrm{H} \\
15 \mathrm{H} \\
2 \mathrm{H}\end{array}$ & $\begin{array}{l}11938.54 \\
11928.80 \\
11907.35 \\
11887.51 \\
11871.65\end{array}$ & $\begin{array}{r}-0.09 \\
0.02 \\
-0.09 \\
-0.14 \\
0.01\end{array}$ & $\begin{array}{c}z^{4} \mathrm{~F}^{\circ}{ }_{31 / 2}-e^{4} \mathrm{D}_{31 / 2} \\
b{ }^{4} \mathrm{P}_{21 / 2}-z^{4} \mathrm{D}_{31 / 2}^{\circ} \\
e^{6} \mathrm{~S}_{21 / 2}-t^{6} \mathrm{P}_{11 / 2}^{0} \\
e^{6} \mathrm{~S}_{21 / 2}-t^{6} \mathrm{P}_{21 / 2}^{\circ} \\
z^{4} \mathrm{~F}_{21 / 2}^{0}-i{ }^{6} \mathrm{D}_{11 / 2}^{\circ}\end{array}$ & \\
\hline $\begin{array}{l}8431.20 \\
8476.3 \\
8481.70 \\
8506.0 \\
8521.57\end{array}$ & $\begin{array}{c}20 \mathrm{H} \\
1 \mathrm{H} \\
3 \\
1 \mathrm{H} \\
10 h\end{array}$ & $\begin{array}{l}11857.45 \\
11794.4 \\
11786.85 \\
11753.18 \\
11731.71\end{array}$ & $\begin{array}{r}-0.04 \\
-0.1 \\
-0.05 \\
-0.02 \\
0.01\end{array}$ & $\begin{array}{l}e^{6} \mathrm{~S}_{21 / 2}-t^{6} \mathrm{P}_{31 / 2}^{0} \\
z^{4} \mathrm{~F}_{21 / 2}^{0}-i{ }^{6} \mathrm{D}_{21 / 2} \\
z^{4} \mathrm{~F}_{11 / 2}-e \\
z^{4} \mathrm{D}_{11 / 2} \\
z^{4} \mathrm{~F}_{11 / 2}^{0}-i{ }^{6} \mathrm{D}_{11 / 2} \\
z^{4} \mathrm{D}_{31 / 2}^{0}-f^{4} \mathrm{D}_{21 / 2}\end{array}$ & \\
\hline $\begin{array}{l}8558.63 \\
8600.34 \\
8602.1 \\
8603.03 \\
8654.63\end{array}$ & $\begin{array}{l}8 h \\
5 \mathrm{H} \\
2 \mathrm{H} \\
5 \\
40 h\end{array}$ & $\begin{array}{l}\text { 11680. } 91 \\
11624.26 \\
11621.9 \\
11620.62 \\
11551.34\end{array}$ & $\begin{array}{r}-0.06 \\
-0.07 \\
0.0 \\
0.10 \\
-0.01\end{array}$ & $\begin{array}{c}z{ }^{4} \mathrm{D}_{21 / 2}^{\circ}-f{ }^{4} \mathrm{D}_{11 / 2} \\
z^{6} \mathrm{~F}_{51 / 2}^{\circ}-g^{6} \mathrm{D}_{41 / 2} \\
z^{4} \mathrm{D}_{11 / 2}^{\circ}-f{ }^{4} \mathrm{D}_{01 / 2} \\
b^{4} \mathrm{P}_{11 / 2}-z^{4} \mathrm{D}_{11 / 2}^{\circ} \\
z^{4} \mathrm{D}_{31 / 2}^{0}-f^{4} \mathrm{D}_{31 / 2}\end{array}$ & \\
\hline $\begin{array}{l}8659.38 \\
8664.6 \\
8666.3 \\
8670.92 \\
8672.06\end{array}$ & $\begin{array}{r}10 h \\
2 h \\
2 h \\
200 c \\
300 c\end{array}$ & $\begin{array}{l}11545.00 \\
11538.0 \\
11535.8 \\
11529.74 \\
11528.12\end{array}$ & $\begin{array}{r}-0.04 \\
0.0 \\
-0.1 \\
0.08 \\
-0.05\end{array}$ & 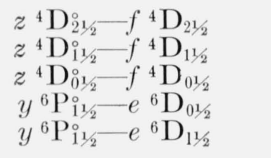 & \\
\hline $\begin{array}{l}8673.97 \\
8680.24 \\
8699.13 \\
8701.05 \\
8703.76\end{array}$ & $\begin{array}{l}200 c \\
2 h \\
100 c \\
300 c \\
500 c w\end{array}$ & $\begin{array}{l}11525.58 \\
11517.26 \\
11492.25 \\
11489.71 \\
11486.14\end{array}$ & $\begin{array}{l}-0.05 \\
-0.25 \\
-0.05 \\
-0.05 \\
-0.07\end{array}$ & $\begin{array}{l}y^{6} \mathrm{P}_{11 / 2}^{\circ}-e{ }^{6} \mathrm{D}_{21 / 2} \\
z^{6} \mathrm{~F}_{41 / 2}^{\circ}-g^{6} \mathrm{D}_{31 / 2} \\
y^{6} \mathrm{P}_{21 / 2}^{o}-e{ }^{6} \mathrm{D}_{11 / 2} \\
y^{6} \mathrm{P}_{21 / 2}^{o}-e{ }^{6} \mathrm{D}_{21 / 2} \\
y^{6} \mathrm{P}_{21 / 2}^{o}-e{ }^{6} \mathrm{D}_{31 / 2}\end{array}$ & \\
\hline $\begin{array}{l}8710.21 \\
8717.29 \\
8729.80 \\
8734.60 \\
8737.32\end{array}$ & $\begin{array}{c}10 \\
2 h \\
2 h \\
30 c \\
300 c\end{array}$ & $\begin{array}{l}11477.63 \\
11468.31 \\
11451.80 \\
11445.58 \\
11442.02\end{array}$ & $\begin{array}{r}0.07 \\
0.15 \\
-0.17 \\
-0.06 \\
-0.07\end{array}$ & $\begin{array}{l}b{ }^{4} \mathrm{P}_{11 / 2}-z^{4} \mathrm{D}_{21 / 2}^{\circ} \\
x^{6} \mathrm{P}_{31 / 2}^{\circ}-e^{4} \mathrm{D}_{31 / 2} \\
z^{4} \mathrm{D}_{01 / 2}^{\circ}-f^{4} \mathrm{D}_{11 / 2} \\
y^{6} \mathrm{P}_{31 / 2}^{\circ}-e^{6} \mathrm{D}_{21 / 2} \\
y^{6} \mathrm{P}_{31 / 2}^{\circ}-e^{6} \mathrm{D}_{31 / 2}\end{array}$ & \\
\hline $\begin{array}{l}8740.93 \\
8767.96 \\
8796.83 \\
8798.66 \\
8820.26\end{array}$ & $\begin{array}{c}1000 c w \\
5 h \\
6 h \\
3 h \\
4 h\end{array}$ & $\begin{array}{l}11437.29 \\
11402.03 \\
11364.61 \\
11362.25 \\
11334.43\end{array}$ & $\begin{array}{r}-0.02 \\
-0.05 \\
-0.08 \\
\left\{\begin{array}{r}-0.04 \\
0.10 \\
-0.25\end{array}\right.\end{array}$ & $\begin{array}{l}y^{6} \mathrm{P}_{31 / 2}^{0}-e{ }^{6} \mathrm{D}_{41 / 2} \\
z^{4} \mathrm{D}_{11 / 2}^{\circ}-f^{4} \mathrm{D}_{21 / 2} \\
z^{4} \mathrm{D}_{21 / 2}-f^{4} \mathrm{D}_{31 / 2} \\
x^{6} \mathrm{P}_{31 / 2}^{\circ}-i{ }^{6} \mathrm{D}_{31 / 2} \\
y^{8} \mathrm{P}_{41 / 2}-e^{8} \mathrm{P}_{41 / 2} \\
x^{6} \mathrm{P}_{21 / 2}^{0}-i{ }^{6} \mathrm{D}_{21 / 2}\end{array}$ & \\
\hline $\begin{array}{l}8827.83 \\
8842.48 \\
8895.9 \\
8901.0 \\
8926.06\end{array}$ & $\begin{array}{l}3 \\
3 h \\
4 \\
2 p \\
15 h\end{array}$ & $\begin{array}{l}11324.70 \\
11305.94 \\
11238.72 \\
11231.6 \\
11200.08\end{array}$ & $\begin{aligned} 0.03 \\
-0.03 \\
0.09 \\
0.0 \\
-0.02\end{aligned}$ & $\begin{array}{l}b{ }^{4} \mathrm{P}_{01 / 2}-z{ }^{4} \mathrm{D}_{01 / 2}^{\circ}{ }^{\circ} \\
x{ }^{6} \mathrm{P}_{21 / 2}^{\circ}-e^{4} \mathrm{D}_{31 / 2} \\
b^{4} \mathrm{P}_{01 / 2}-z \\
{ }^{4} \mathrm{D}_{11 / 2}^{\circ} \\
x{ }^{6} \mathrm{P}_{11 / 2}-i{ }^{6} \mathrm{D}_{21 / 2} \\
x^{6} \mathrm{P}_{21 / 2}^{\circ}-i{ }^{6} \mathrm{D}_{31 / 2}\end{array}$ & \\
\hline
\end{tabular}


TaBle 1. Mn I-Classified lines-Continued

\begin{tabular}{|c|c|c|c|c|c|}
\hline 1 & 2 & 3 & 4 & 5 & 6 \\
\hline$\lambda$ & Int. & $\sigma$ & $\mathrm{o}-\mathrm{c}$ & Term designation & $\begin{array}{l}\text { Zeeman } \\
\text { type }\end{array}$ \\
\hline 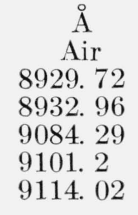 & $\begin{array}{l}60 h \\
2 \\
30 \\
3 h w \\
50\end{array}$ & \begin{tabular}{l}
\multicolumn{1}{c}{$K$} \\
11195. 49 \\
11191.43 \\
11005.00 \\
10984.6 \\
10969.10
\end{tabular} & $\begin{array}{l}K \\
-0.04 \\
0.03 \\
0.12 \\
0.0 \\
0.19\end{array}$ & $\begin{array}{l}x^{6} \mathrm{P}_{31 / 2}^{\circ}-i{ }^{6} \mathrm{D}_{41 / 2} \\
y^{8} \mathrm{P}_{41 / 2}^{\circ}-e^{8} \mathrm{P}_{31 / 2} \\
a^{4} \mathrm{~F}_{11 / 2}-z^{4} \mathrm{D}_{01 / 2}^{\circ} \\
e^{8} \mathrm{D}_{51 / 2}-t^{6} \mathrm{~F}^{0} \\
a^{4} \mathrm{~F}_{21 / 2}-z^{4} \mathrm{D}_{11 / 2}^{\circ}\end{array}$ & \\
\hline $\begin{array}{l}9155.85 \\
9172.09 \\
9234.40 \\
9243.29 \\
9325.16\end{array}$ & $\begin{array}{r}5 \\
100 \\
10 \\
150 \\
5\end{array}$ & $\begin{array}{l}\text { 10918. } 98 \\
10899.66 \\
10826.11 \\
10815.69 \\
10720.74\end{array}$ & $\begin{array}{r}0.14 \\
0.10 \\
0.16 \\
0.12 \\
-0.03\end{array}$ & $\begin{array}{l}a^{4} \mathrm{~F}_{11 / 2}-z^{4} \mathrm{D}_{11 / 2}^{\circ} \\
a^{4} \mathrm{~F}_{31 / 2}-z^{4} \mathrm{D}_{21 / 2}^{\circ} \\
a^{4} \mathrm{~F}_{21 / 2}-z^{4} \mathrm{D}_{21 / 2}^{\circ} \\
a^{4} \mathrm{~F}_{41 / 2}-z^{4} \mathrm{D}_{31 / 2}^{0} \\
y^{4} \mathrm{P}_{21 / 2}^{0}-f^{4} \mathrm{D}_{11 / 2}^{\circ}\end{array}$ & \\
\hline $\begin{array}{l}9331.90 \\
9336.47 \\
9412.78 \\
9429.58 \\
9444.90\end{array}$ & $\begin{array}{l}20 h \\
40 h \\
10 h \\
30 h \\
40\end{array}$ & $\begin{array}{l}10712.99 \\
10707.75 \\
10620.94 \\
10602.02 \\
10584.82\end{array}$ & $\begin{array}{r}0.09 \\
-0.06 \\
-0.08 \\
0.08 \\
-0.02\end{array}$ & $\begin{array}{l}a^{4} \mathrm{~F}_{31 / 2}-z{ }^{4} \mathrm{D}_{31 / 2}^{\circ} \\
z^{4} \mathrm{D}_{31 / 2}^{\circ}-e{ }^{4} \mathrm{D}_{31 / 2}^{\circ} \\
z^{4} \mathrm{D}_{21 / 2}^{\circ}-e{ }^{4} \mathrm{D}_{21 / 2}^{\circ} \\
z^{4} \mathrm{D}_{31 / 2}^{\circ}-i{ }^{6} \mathrm{D}_{31 / 2}^{\circ} \\
y^{4} \mathrm{P}_{21 / 2}^{0}-f^{4} \mathrm{D}_{21 / 2}\end{array}$ & \\
\hline $\begin{array}{l}9474.9 \\
9476.57 \\
9502.12 \\
9335.72 \\
9550.80\end{array}$ & $\begin{array}{r}2 \\
4 h \\
6 h \\
5 h \\
20 h\end{array}$ & $\begin{array}{l}\text { 10551. } 3 \\
10549.45 \\
10521.08 \\
10484.01 \\
10467.46\end{array}$ & $\begin{array}{l}0.0 \\
-0.41 \\
-0.07 \\
-0.03 \\
0.07\end{array}$ & $\begin{array}{l}y{ }^{4} \mathrm{P}_{11 / 2}^{\circ}-f^{4} \mathrm{D}_{01 / 2} \\
z^{4} \mathrm{D}_{21 / 2}^{\circ}-i{ }^{6} \mathrm{D}_{21 / 2}\end{array}$ & \\
\hline $\begin{array}{l}9584.0 \\
9598.7 \\
9606.71 \\
9608.56 \\
9676.50\end{array}$ & $\begin{array}{c}10 h \\
3 \\
5 h \\
100 h \\
40\end{array}$ & $\begin{array}{l}\text { 10431. } 2 \\
10415.2 \\
10406.54 \\
10404.54 \\
10331.49\end{array}$ & $\begin{array}{r}-0.5 \\
-0.1 \\
0.00 \\
0.05 \\
0.03\end{array}$ & $\begin{array}{l}z{ }^{4} \mathrm{D}_{01 / 2}^{\circ}-e{ }^{4} \mathrm{D}_{11 / 2} \\
z^{4} \mathrm{D}_{21 / 2}^{\circ}-i{ }^{6} \mathrm{D}_{31 / 2} \\
y{ }^{4} \mathrm{P}_{01 / 2}^{0}-f{ }^{4} \mathrm{D}_{01 / 2} \\
y{ }^{4} \mathrm{P}_{21 / 2}^{\circ}-f{ }^{4} \mathrm{D}_{31 / 2} \\
y{ }^{4} \mathrm{P}_{11 / 2}^{0}-f{ }^{4} \mathrm{D}_{21 / 2}\end{array}$ & \\
\hline $\begin{array}{r}9684.9 \\
9845.1 \\
10045.4 \\
10053.1 \\
10164.9\end{array}$ & $\begin{array}{l}15 \\
5 \mathrm{H} \\
4 \mathrm{H} \\
10 \mathrm{H} \\
2 h\end{array}$ & $\begin{array}{r}10322.5 \\
10154.54 \\
9952.08 \\
9944.46 \\
9835.08\end{array}$ & $\begin{array}{r}-0.1 \\
0.0 \\
-0.09 \\
-0.18 \\
-0.15\end{array}$ & $\begin{array}{c}y{ }^{4} \mathrm{P}_{01 / 2}^{\circ}-f^{4} \mathrm{D}_{11 / 2} \\
e^{8} \mathrm{D}_{51 / 2}-u{ }^{6} \mathbf{F}^{\circ} \\
x^{6} \mathrm{P}_{31 / 2}^{\circ}-g \\
{ }^{6} \mathrm{D}_{31 / 2} \\
x^{6} \mathrm{P}_{31 / 2}^{\circ}-g \\
{ }^{6} \mathrm{D}_{41 / 2} \\
b^{2} \mathrm{I}_{51 / 2}-z^{8} \mathbf{F}^{\circ} ?\end{array}$ & \\
\hline $\begin{array}{l}\text { 10204. } 9 \\
10208.2 \\
10212.3 \\
10300.7 \\
10313.35\end{array}$ & $\begin{array}{l}1 \mathrm{H} \\
2 \mathrm{H} \\
5 \mathrm{H} \\
5 h \\
2 h\end{array}$ & $\begin{array}{l}9796.53 \\
9793.36 \\
9789.43 \\
9705.42 \\
9693.51\end{array}$ & $\begin{array}{l}-0.38 \\
-1.19 \\
-0.55 \\
-0.11 \\
-0.34\end{array}$ & $\begin{array}{l}x{ }^{6} \mathrm{P}_{21 / 2}^{0}-g{ }^{6} \mathrm{D}_{11 / 2} \\
x{ }^{6} \mathrm{P}_{21 / 2}^{\circ}-g \\
{ }^{6} \mathrm{D}_{21 / 2}\end{array}$ & \\
\hline $\begin{array}{l}\text { 10316. } 05 \\
10349.3 \\
10456.60 \\
10561.2 \\
10621.9\end{array}$ & $\begin{array}{l}5 h \\
2 \mathrm{H} \\
3 \\
6 h \\
6\end{array}$ & $\begin{array}{l}9690.97 \\
9659.84 \\
9560.72 \\
9465.94 \\
9411.93\end{array}$ & $\begin{array}{l}0.24 \\
0.0 \\
-0.23 \\
-0.44 \\
-0.23\end{array}$ & $\begin{array}{l}x{ }^{6} \mathrm{P}_{11 / 2}^{\circ}-g{ }^{6} \mathrm{D}_{01 / 2} \\
e{ }^{6} \mathrm{D}_{41 / 2}-u \\
{ }^{6} \mathrm{~F}^{0} \\
y^{4} \mathrm{P}_{21 / 2}^{0}-e^{4} \mathrm{D}_{31 / 2} \\
x^{6} \mathrm{P}_{31 / 2}^{\circ}-h{ }^{6} \mathrm{~S}_{21 / 2}\end{array}$ & \\
\hline $\begin{array}{l}\text { 10643. } 0 \\
10664.1 \\
10686.8 \\
10692.2 \\
10745.32\end{array}$ & $\begin{array}{l}10 \mathrm{H} \\
15 \mathrm{H} \\
2 \\
20 \mathrm{H} \\
6 h\end{array}$ & $\begin{array}{l}\text { 9393. } 26 \\
9374.69 \\
9354.78 \\
9350.05 \\
9303.83\end{array}$ & $\begin{array}{r}-0.06 \\
-0.24 \\
-0.17 \\
0.10 \\
-0.36\end{array}$ & 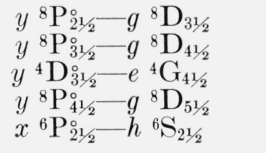 & \\
\hline $\begin{array}{l}\text { 10856. } 08 \\
10865.85 \\
10938.65 \\
11163.3 \\
11378.8\end{array}$ & $\begin{array}{c}2 h \\
2 h \\
3 h \\
2 \\
100 w\end{array}$ & $\begin{array}{l}9208.91 \\
9200.63 \\
9139.40 \\
8955.47 \\
8785.89\end{array}$ & $\begin{array}{l}-0.23 \\
-0.50 \\
-0.11 \\
-0.04 \\
0.0\end{array}$ & $\begin{array}{l}z^{6} \mathrm{~F}_{31 / 2}^{\circ}-f^{6} \mathrm{D}_{21 / 2} \\
x^{6} \mathrm{P}_{11 / 2}^{\circ}-h{ }^{6} \mathrm{~S}_{21 / 2} \\
z^{6} \mathrm{~F}_{21 / 2}^{\circ}-f^{6} \mathrm{D}_{11 / 2} \\
x^{6} \mathrm{D}_{41 / 2}^{\circ}-e^{6} \mathrm{~F}_{51 / 2}^{\circ} \\
e^{8} \mathrm{D}_{51 / 2}-y{ }^{8} \mathrm{~F}_{61 / 2}^{\circ}\end{array}$ & \\
\hline $\begin{array}{l}11497.61 \\
11613.24 \\
11644.84 \\
11783.58 \\
12899.7\end{array}$ & $\begin{array}{r}40 \\
50 \\
3 \\
60 \\
80\end{array}$ & $\begin{array}{l}8695.08 \\
8608.50 \\
8585.14 \\
8484.07 \\
7750.0\end{array}$ & $\begin{array}{l}-0.15 \\
-0.10 \\
-0.24 \\
-0.08 \\
0.0\end{array}$ & 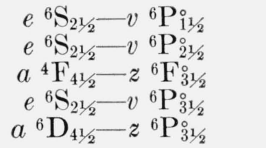 & \\
\hline
\end{tabular}


Table 1. Mn I-Classified lines-Continued

\begin{tabular}{|c|c|c|c|c|c|}
\hline 1 & 2 & 3 & 4 & 5 & 6 \\
\hline$\lambda$ & Int. & $\sigma$ & $o-c$ & Term designation & $\begin{array}{c}\text { Zeeman } \\
\text { type }\end{array}$ \\
\hline $\begin{array}{c}\AA \\
\text { Air } \\
\text { 12975. } 9 \\
13294.1 \\
13317.9 \\
\text { 13415. } 9 \\
\text { 13500. } 1\end{array}$ & $\begin{array}{r}40 \\
50 \\
30 \\
80 \\
100\end{array}$ & $\begin{array}{c}K \\
7704.5 \\
7520.1 \\
7506.7 \\
7451.8 \\
7405.4\end{array}$ & $\begin{array}{r}K \\
0.0 \\
-0.1 \\
0.7 \\
-0.2 \\
0.0\end{array}$ & $\begin{array}{l}a^{4} \mathrm{D}_{31 / 2}-z{ }^{4} \mathrm{P}_{21 / 2}^{0} \\
a{ }^{6} \mathrm{D}_{31 / 2}-z \\
{ }^{6} \mathrm{P}_{31 / 2}^{0} \\
a^{6} \mathrm{D}_{31 / 2}-z \\
{ }^{6} \mathrm{P}_{21 / 2}^{0} \\
a^{4} \mathrm{D}_{21 / 2}-z^{4} \mathrm{P}_{21 / 2}^{0} \\
a^{4} \mathrm{D}_{11 / 2}-z^{4} \mathrm{P}_{01 / 2}^{01}\end{array}$ & \\
\hline $\begin{array}{l}13625.7 \\
13684.6 \\
13863.8 \\
13997.0 \\
14969.9\end{array}$ & $\begin{array}{r}200 \\
80 \\
100 \\
120 \\
30\end{array}$ & $\begin{array}{l}7337.1 \\
7305.5 \\
7211.1 \\
7142.4 \\
6678.2\end{array}$ & $\begin{array}{r}0.6 \\
-0.6 \\
0.3 \\
0.2 \\
-0.3\end{array}$ & $\begin{array}{l}a^{6} \mathrm{D}_{21 / 2}-z^{6} \mathrm{P}_{21 / 2}^{0} \\
a^{4} \mathrm{D}_{01 / 2}-z^{4} \mathrm{P}_{01 / 2}^{\circ} \\
a^{6} \mathrm{D}_{11 / 2}-z^{6} \mathrm{P}_{11 / 2}^{0} \\
a^{6} \mathrm{D}_{01 / 2}-z^{6} \mathrm{P}_{11 / 2}^{\circ} \\
y^{8} \mathrm{P}_{41 / 2}^{0}-f^{8} \mathrm{D}_{51 / 2}\end{array}$ & \\
\hline $\begin{array}{l}15217.9 \\
15263.1 \\
15964.9 \\
17335.2 \\
17607.5\end{array}$ & $\begin{array}{r}80 \\
200 \\
200 \\
80 \\
20\end{array}$ & $\begin{array}{l}6569.4 \\
6550.0 \\
6262.1 \\
5767.0 \\
5677.9\end{array}$ & $\begin{array}{r}-0.1 \\
-0.1 \\
0.2 \\
-0.2 \\
-0.2\end{array}$ & 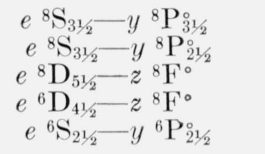 & \\
\hline
\end{tabular}

TABLE 2. Even terms of Mn I

\begin{tabular}{|c|c|c|c|c|c|}
\hline Config. & Desig. & $J$ & Level & Interval & Obs. g \\
\hline $3 d^{5} 4 s^{2}$ & $a^{6} \mathrm{~S}$ & $21 / 2$ & 0. 00 & & 1. 999 \\
\hline $3 d^{6}\left(a^{5} \mathrm{D}\right) 4 s$ & $a^{6} \mathrm{D}$ & $\begin{array}{l}41 / 2 \\
31 / 2 \\
21 / 2 \\
11 / 2 \\
01 / 2\end{array}$ & $\begin{array}{l}17052.29 \\
17282.00 \\
17451.52 \\
17568.48 \\
17637.15\end{array}$ & $\begin{array}{r}-229.71 \\
-169.52 \\
-116.96 \\
-68.67\end{array}$ & $\begin{array}{l}\text { 1. } 559 \\
\text { 1. } 584 \\
\text { 1. } 657 \\
\text { 1. } 866 \\
\text { 3. } 327\end{array}$ \\
\hline $3 d^{6}\left(a{ }^{5} \mathrm{D}\right) 4 s$ & $a{ }^{4} \mathrm{D}$ & $\begin{array}{l}31 / 2 \\
21 / 2 \\
11 / 2 \\
01 / 2\end{array}$ & $\begin{array}{l}23296.67 \\
23549.20 \\
23719.52 \\
23818.87\end{array}$ & $\begin{array}{r}-252.53 \\
-170.32 \\
-99.35\end{array}$ & $\begin{array}{l}\text { 1. } 427 \\
\text { 1. } 368 \\
\text { 1. } 198 \\
0.000\end{array}$ \\
\hline $3 d^{5} 4 s^{2}$ & $a^{4} \mathrm{G}$ & $\begin{array}{l}51 / 2 \\
41 / 2 \\
31 / 2 \\
21 / 2\end{array}$ & $\begin{array}{l}25265.74 \\
25285.43 \\
25287.74 \\
25281.04\end{array}$ & $\begin{array}{r}-19.69 \\
-2.31 \\
6.70\end{array}$ & $\begin{array}{l}\text { 1. } 270 \\
\text { 1. } 173\end{array}$ \\
\hline $3 d^{5} 4 s^{2}$ & $a{ }^{4} \mathrm{P}$ & $\begin{array}{l}21 / 2 \\
11 / 2 \\
01 / 2\end{array}$ & $\begin{array}{l}27201.54 \\
27248.00 \\
27281.85\end{array}$ & $\begin{array}{l}-46.46 \\
-33.85\end{array}$ & $\begin{array}{l}\text { 1. } 597 \\
\text { 1. } 730 \\
\text { 2. } 666\end{array}$ \\
\hline $3 d^{5} 4 s^{2}$ & $b{ }^{4} \mathrm{D}$ & $\begin{array}{l}31 / 2 \\
21 / 2 \\
11 / 2 \\
01 / 2\end{array}$ & $\begin{array}{l}\text { 30354. } 21 \\
30419.61 \\
30425.71 \\
30411.74\end{array}$ & $\begin{array}{r}-65.40 \\
-6.10 \\
13.97\end{array}$ & $\begin{array}{l}\text { 1. } 425 \\
\text { 1. } 38 \\
\text { 0. } 111\end{array}$ \\
\hline $3 d^{6}\left(a^{3} \mathrm{P}\right) 4 s$ & $b{ }^{4} \mathrm{P}$ & $\begin{array}{l}21 / 2 \\
11 / 2 \\
01 / 2\end{array}$ & $\begin{array}{l}33825.49 \\
34463.37 \\
34845.26\end{array}$ & $\begin{array}{l}-637.88 \\
-381.89\end{array}$ & $\begin{array}{l}\text { 1. } 602 \\
\text { 1. } 730 \\
\text { 2. } 655\end{array}$ \\
\hline $3 d^{6}\left(a^{3} \mathrm{H}\right) 4 s$ & $a{ }^{4} \mathrm{H}$ & $\begin{array}{l}61 / 2 \\
51 / 2 \\
41 / 2 \\
31 / 2\end{array}$ & $\begin{array}{l}34138.88 \\
34250.52 \\
34343.90 \\
34423.27\end{array}$ & $\begin{array}{r}-111.64 \\
-93.38 \\
-79.37\end{array}$ & $\begin{array}{l}\text { 1. } 231 \\
\text { 1. } 135 \\
0.971 \\
0.665\end{array}$ \\
\hline
\end{tabular}


Table 2. Even terms of Mn I-Continued

\begin{tabular}{|c|c|c|c|c|c|}
\hline Config. & Desig. & $J$ & Level & Interval & Obs. g \\
\hline $3 d^{6}\left(a^{3} \mathrm{~F}\right) 4 s$ & $a^{4} \mathrm{~F}$ & $\begin{array}{l}41 / 2 \\
31 / 2 \\
21 / 2 \\
11 / 2\end{array}$ & $\begin{array}{l}34938.70 \\
35041.37 \\
35114.98 \\
35165.05\end{array}$ & $\begin{array}{r}-102.67 \\
-73.61 \\
-50.07\end{array}$ & $\begin{array}{l}\text { 1. } 328 \\
\text { 1. } 238 \\
\text { 1. } 024 \\
0.430\end{array}$ \\
\hline $3 d^{5} 4 s^{2}$ & $a^{2} \mathrm{I}$ & $\begin{array}{l}51 / 2 \\
61 / 2\end{array}$ & $\begin{array}{l}37148.66 \\
37164.25\end{array}$ & 15. 59 & 0. 94 \\
\hline $3 d^{6}\left(\mathrm{a}^{3} \mathrm{G}\right) 4 s$ & $b^{4} \mathrm{G}$ & $\begin{array}{l}51 / 2 \\
41 / 2 \\
31 / 2 \\
21 / 2\end{array}$ & $\begin{array}{l}37420.24 \\
37630.62 \\
37737.22 \\
37789.93\end{array}$ & $\begin{array}{r}-210.58 \\
-106.40 \\
-52.71\end{array}$ & $\begin{array}{l}\text { 1. } 263 \\
\text { 1. } 163 \\
0.989 \\
0.59\end{array}$ \\
\hline $3 d^{6}\left({ }^{3} \mathrm{P}\right) 4 s$ & $a^{2} \mathrm{P}$ & $\begin{array}{l}11 / 2 \\
01 / 2\end{array}$ & $\begin{array}{l}37586.03 \\
38351.78\end{array}$ & -765.75 & 0.675 \\
\hline $3 d^{6}\left(a^{3} \mathrm{H}\right) 4 s$ & $a^{2} \mathrm{H}$ & $\begin{array}{l}51 / 2 \\
41 / 2\end{array}$ & $\begin{array}{l}38008.70 \\
38120.18\end{array}$ & -111.48 & $\begin{array}{l}\text { 1. } 098 \\
\text { 0. } 914\end{array}$ \\
\hline $3 d^{6}\left(a^{3} \mathrm{~F}\right) 4 s$ & $a^{2} \mathrm{~F}$ & $\begin{array}{l}31 / 2 \\
21 / 2\end{array}$ & $\begin{array}{l}38669.60 \\
38934.94\end{array}$ & -265.34 & 1. 128 \\
\hline $3 d^{5} 4 s\left(a^{7} \mathrm{~S}\right) 5 s$ & $e^{8} \mathrm{~S}$ & $31 / 2$ & 39431. 31 & & 2. 000 \\
\hline $3 d^{6}\left({ }^{3} \mathrm{G}\right) 4 s$ & $a^{2} \mathrm{G}$ & $\begin{array}{l}41 / 2 \\
31 / 2\end{array}$ & $\begin{array}{l}41031.48 \\
41230.30\end{array}$ & -198.86 & $\begin{array}{l}\text { 1. } 118 \\
0.88\end{array}$ \\
\hline \multirow[t]{2}{*}{$3 d^{5} 4 s\left(a^{7} \mathrm{~S}\right) 5 s$} & $e^{6} \mathrm{~S}$ & $21 / 2$ & 41403. 93 & & 1. 997 \\
\hline & $b^{2} \mathrm{I}$ & $\begin{array}{l}61 / 2 \\
51 / 2\end{array}$ & $\begin{array}{l}\text { 43053. } 30 \\
43139.27\end{array}$ & -85.97 & $\begin{array}{l}\text { 1. } 07 \\
0.924\end{array}$ \\
\hline $3 d^{5} 4 s\left(a^{7} \mathrm{~S}\right) 4 d$ & $e^{8} \mathrm{D}$ & $\begin{array}{l}11 / 2 \\
21 / 2 \\
31 / 2 \\
41 / 2 \\
51 / 2\end{array}$ & $\begin{array}{l}46706.09 \\
46707.03 \\
46708.33 \\
46710.15 \\
46712.58\end{array}$ & $\begin{array}{l}0.94 \\
\text { 1. } 30 \\
\text { 1. } 82 \\
\text { 2. } 43\end{array}$ & \\
\hline $3 d^{5} 4 s\left(a^{7} \mathrm{~S}\right) 4 d$ & $e{ }^{6} \mathrm{D}$ & $\begin{array}{l}41 / 2 \\
31 / 2 \\
21 / 2 \\
11 / 2 \\
01 / 2\end{array}$ & $\begin{array}{l}47207.28 \\
47212.06 \\
47215.61 \\
47218.15 \\
47219.64\end{array}$ & $\begin{array}{l}-4.78 \\
-3.55 \\
-2.54 \\
-1.49\end{array}$ & $\begin{array}{l}\text { 1. } 554 \\
\text { 1. } 581 \\
\text { 1. } 634 \\
\text { 1. } 759 \\
\text { 3. } 934\end{array}$ \\
\hline $3 d^{5} 4 s\left(a^{5} \mathrm{~S}\right) 5 s$ & $f^{6} \mathrm{~S}$ & $21 / 2$ & 49415. 35 & & 2. 00 \\
\hline $3 d^{5} 4 s\left(a^{5} \mathrm{~S}\right) 5 s$ & $e^{4} \mathrm{~S}$ & $11 / 2$ & 49591.51 & & 1. 998 \\
\hline $3 d^{5} 4 s\left(a^{7} \mathrm{~S}\right) 6 s$ & $f^{8} \mathrm{~S}$ & $31 / 2$ & 50157.63 & & 1. 995 \\
\hline $3 d^{5} 4 s\left(a^{7} \mathrm{~S}\right) 6 s$ & $g{ }^{6} \mathrm{~S}$ & $21 / 2$ & 50904.68 & & \\
\hline $3 d^{7}$ & $e^{4} \mathrm{P}$ & $\begin{array}{l}21 / 2 \\
11 / 2 \\
01 / 2\end{array}$ & $\begin{array}{l}51638.17 \\
51718.22 \\
51787.92\end{array}$ & $\begin{array}{l}-80.05 \\
-69.70\end{array}$ & $\begin{array}{l}\text { 1. } 601 \\
\text { 1. } 733 \\
\text { 2. } 65\end{array}$ \\
\hline $3 d^{5} 4 s\left(a^{7} \mathrm{~S}\right) 5 d$ & $f^{8} \mathrm{D}$ & $\begin{array}{l}11 / 2 \\
21 / 2 \\
31 / 2 \\
41 / 2 \\
51 / 2\end{array}$ & $\begin{array}{l}52702.48 ? \\
52703.1 \\
52705.23\end{array}$ & $\begin{array}{l}0.6 \\
2.1\end{array}$ & \\
\hline $3 d^{5} 4 s\left(a^{7} \mathrm{~S}\right) 5 d$ & $f^{6} \mathrm{D}$ & $\begin{array}{l}41 / 2 \\
31 / 2 \\
21 / 2 \\
11 / 2 \\
01 / 2\end{array}$ & $\begin{array}{l}52726.39 \\
52730.41 \\
52733.22 \\
52735.01 \\
52735.83\end{array}$ & $\begin{array}{l}-4.02 \\
-2.81 \\
-1.79 \\
-0.82\end{array}$ & \\
\hline $3 d^{5} 4 s\left(a^{7} \mathbf{S}\right) 7 s$ & $h{ }^{6} \mathrm{~S}$ & $21 / 2$ & 54460.30 & & \\
\hline
\end{tabular}


TABle 2. Even terms of Mn I-Continued

\begin{tabular}{|c|c|c|c|c|c|}
\hline Config. & Desig. & $J$ & Level & Interval & Obs. g \\
\hline $3 d^{5} 4 s\left(a{ }^{5} \mathrm{~S}\right) 4 d$ & $g{ }^{6} \mathrm{D}$ & $\begin{array}{l}41 / 2 \\
31 / 2 \\
21 / 2 \\
11 / 2 \\
01 / 2\end{array}$ & $\begin{array}{l}54938.56 \\
54946.09 \\
54950.66 \\
54953.02 \\
54949.90\end{array}$ & $\begin{array}{r}-7.53 \\
-4.57 \\
-2.36 \\
3.12\end{array}$ & \\
\hline $3 d^{5} 4 s\left(a^{7} \mathrm{~S}\right) 6 d$ & $g^{8} \mathrm{D}$ & $\begin{array}{l}11 / 2 \\
21 / 2 \\
31 / 2 \\
41 / 2 \\
51 / 2\end{array}$ & $\begin{array}{l}55374.76 \\
55375.70 \\
55376.70\end{array}$ & $\begin{array}{l}0.94 \\
1.00\end{array}$ & \\
\hline $3 d^{5} 4 s\left(a^{7} \mathrm{~S}\right) 6 d$ & $h{ }^{6} \mathrm{D}$ & $\begin{array}{l}41 / 2 \\
31 / 2 \\
21 / 2 \\
11 / 2 \\
01 / 2\end{array}$ & $\begin{array}{l}55681.90 \\
55688.10 \\
55690.80 \\
55691.90 \\
55692.40\end{array}$ & $\begin{array}{l}-6.20 \\
-2.70 \\
-1.10 \\
-0.50\end{array}$ & \\
\hline $3 d^{5} 4 s\left(a^{7} \mathrm{~S}\right) 8 s$ & $g^{8} \mathrm{~S}$ & $31 / 2$ & 56144.16 & & \\
\hline \multirow[t]{2}{*}{$3 d^{6}\left(a^{5} \mathrm{D}\right) 5 s$} & $i{ }^{6} \mathrm{D}$ & $\begin{array}{l}41 / 2 \\
31 / 2 \\
21 / 2 \\
11 / 2 \\
01 / 2\end{array}$ & $\begin{array}{l}56189.45 \\
56356.21 \\
56490.79 \\
56567.93 \\
56666.06\end{array}$ & $\begin{array}{r}-166.76 \\
-134.58 \\
-77.14 \\
-98.13\end{array}$ & 1. 57 \\
\hline & $e{ }^{4} \mathrm{D}$ & $\begin{array}{l}31 / 2 \\
21 / 2 \\
11 / 2 \\
01 / 2\end{array}$ & $\begin{array}{l}56462.08 \\
56561.95 \\
56601.63\end{array}$ & $\begin{array}{l}-99.87 \\
-39.68\end{array}$ & \\
\hline $3 d^{5} 4 s\left(a^{7} \mathrm{~S}\right) 7 d$ & $h^{8} \mathrm{D}$ & $\begin{array}{l}11 / 2 \\
\text { to } \\
51 / 2\end{array}$ & 56801. $4 ?$ & & \\
\hline $3 d^{5} 4 p^{2}$ & $e^{8} \mathrm{P}$ & $\begin{array}{l}21 / 2 \\
31 / 2 \\
41 / 2\end{array}$ & $\begin{array}{l}57086.33 \\
57218.15 \\
57388.90\end{array}$ & $\begin{array}{l}131.82 \\
170.75\end{array}$ & $\begin{array}{l}\text { 2. } 27 \\
\text { 1. } 767\end{array}$ \\
\hline $3 d^{6}\left(a^{5} \mathrm{D}\right) 5 s$ & $f^{4} \mathrm{D}$ & $\begin{array}{l}31 / 2 \\
21 / 2 \\
11 / 2 \\
01 / 2\end{array}$ & $\begin{array}{l}57305.62 \\
57485.97 \\
57621.90 \\
57705.83\end{array}$ & $\begin{array}{r}-180.35 \\
-135.93 \\
-83.93\end{array}$ & 1. 372 \\
\hline $\operatorname{Mn}$ II $\left({ }^{7} S_{3}\right)$ & Limit & & 59970 & & \\
\hline $3 d^{6}\left(a^{5} \mathrm{D}\right) 5 d$ & $e^{6} \mathrm{~F}$ & $\begin{array}{l}51 / 2 \\
41 / 2 \\
31 / 2 \\
21 / 2 \\
11 / 2 \\
01 / 2\end{array}$ & $\begin{array}{l}61713.62 \\
62030.18 \\
62294.66 \\
62905.81 \\
63083.24\end{array}$ & $\begin{array}{l}-316.56 \\
-264.48 \\
-611.15 \\
-177.43\end{array}$ & \\
\hline $3 d^{6}\left(a^{5} \mathrm{D}\right) 4 d$ & $e^{6} \mathrm{G}$ & $\begin{array}{l}61 / 2 \\
51 / 2 \\
41 / 2 \\
31 / 2 \\
21 / 2 \\
11 / 2\end{array}$ & $\begin{array}{l}62001.09 \\
62134.45 \\
62300.63 \\
62426.48 \\
62514.59 \\
62573.11\end{array}$ & $\begin{array}{r}-133.36 \\
-166.18 \\
-125.85 \\
-88.11 \\
-58.52\end{array}$ & \\
\hline $3 d^{6}\left(a^{5} \mathrm{D}\right) 4 d$ & $e^{4} \mathrm{G}$ & $\begin{array}{l}51 / 2 \\
41 / 2 \\
31 / 2 \\
21 / 2\end{array}$ & $\begin{array}{l}62295.36 \\
62479.04 \\
62632.77 \\
62753.37\end{array}$ & $\begin{array}{l}-183.68 \\
-153.73 \\
-120.60\end{array}$ & \\
\hline $3 d^{6}\left(a^{5} \mathrm{D}\right) 4 d$ & $e^{4} \mathrm{~F}$ & $\begin{array}{l}41 / 2 \\
31 / 2 \\
21 / 2 \\
11 / 2\end{array}$ & $\begin{array}{l}63231.43 \\
63424.00\end{array}$ & -192.57 & \\
\hline $3 d^{5} 4 s\left(a^{5} \mathrm{G}\right) 5 s$ & $f^{4} \mathrm{G}$ & $\begin{array}{l}51 / 2 \\
41 / 2 \\
31 / 2 \\
21 / 2\end{array}$ & $\begin{array}{l}\text { 68693. } 02 \\
68716.22\end{array}$ & -23.20 & 1. 17 \\
\hline
\end{tabular}


Table 2. Odd Terms of Mn I

\begin{tabular}{|c|c|c|c|c|c|}
\hline Config. & Desig. & $J$ & Level & Interval & Obs. g \\
\hline $3 d^{5} 4 s\left(a^{7} \mathbf{S}\right) 4 p$ & $z^{8} \mathrm{P}^{\circ}$ & $\begin{array}{l}21 / 2 \\
31 / 2 \\
41 / 2\end{array}$ & $\begin{array}{l}18402.46 \\
18531.64 \\
18705.37\end{array}$ & $\begin{array}{l}129.18 \\
173.73\end{array}$ & $\begin{array}{l}\text { 2. } 284 \\
\text { 1. } 938 \\
\text { 1. } 779\end{array}$ \\
\hline $3 d^{5} 4 s\left(a^{7} \mathrm{~S}\right) 4 p$ & $z^{6} \mathrm{P}^{\circ}$ & $\begin{array}{l}11 / 2 \\
21 / 2 \\
31 / 2\end{array}$ & $\begin{array}{l}24779.32 \\
24788.05 \\
24802.25\end{array}$ & $\begin{array}{r}8.73 \\
14.20\end{array}$ & $\begin{array}{l}2.364 \\
\text { 1. } 875 \\
\text { 1. } 714\end{array}$ \\
\hline $3 d^{5} 4 s\left(a^{5} \mathrm{~S}\right) 4 p$ & $z^{4} \mathrm{P}^{\circ}$ & $\begin{array}{l}21 / 2 \\
11 / 2 \\
01 / 2\end{array}$ & $\begin{array}{l}31001.15 \\
31076.42 \\
31124.95\end{array}$ & $\begin{array}{l}-75.27 \\
-48.53\end{array}$ & $\begin{array}{l}\text { 1. } 60 \\
\text { 1. } 732 \\
\text { 2. } 668\end{array}$ \\
\hline $3 d^{5} 4 s\left(a^{5} \mathrm{~S}\right) 4 p$ & $y{ }^{6} \mathrm{P}^{\circ}$ & $\begin{array}{l}11 / 2 \\
21 / 2 \\
31 / 2\end{array}$ & $\begin{array}{l}35689.98 \\
35725.85 \\
35769.97\end{array}$ & $\begin{array}{l}35.87 \\
44.12\end{array}$ & $\begin{array}{l}\text { 2. } 400 \\
\text { 1. } 886 \\
\text { 1. } 712\end{array}$ \\
\hline $3 d^{6}\left(a^{5} \mathrm{D}\right) 4 p$ & $z^{6} \mathrm{D}^{\circ}$ & $\begin{array}{l}41 / 2 \\
31 / 2 \\
21 / 2 \\
11 / 2 \\
01 / 2\end{array}$ & $\begin{array}{l}41789.48 \\
41932.64 \\
42053.73 \\
42143.57 \\
42198.56\end{array}$ & $\begin{array}{r}-143.16 \\
-121.09 \\
-89.84 \\
-54.99\end{array}$ & $\begin{array}{l}1.556 \\
\text { 1. } 587 \\
\text { 1. } 653 \\
\text { 1. } 867 \\
\text { 3. } 317\end{array}$ \\
\hline $3 d^{6}\left(a^{5} \mathrm{D}\right) 4 p$ & $z^{6} \mathrm{~F}^{\circ}$ & $\begin{array}{l}51 / 2 \\
41 / 2 \\
31 / 2 \\
21 / 2 \\
11 / 2 \\
01 / 2\end{array}$ & $\begin{array}{l}43314.23 \\
43428.58 \\
43524.08 \\
43595.50 \\
43644.45 \\
43672.66\end{array}$ & $\begin{array}{r}-114.35 \\
-95.50 \\
-71.42 \\
-48.95 \\
-28.21\end{array}$ & $\begin{array}{r}1.464 \\
\text { 1. } 431 \\
\text { 1. } 395 \\
\text { 1. } 310 \\
\text { 1. } 068 \\
-0.602\end{array}$ \\
\hline $3 d^{6}\left(a^{5} \mathrm{D}\right) 4 p$ & $z^{4} \mathrm{~F}^{\circ}$ & $\begin{array}{l}41 / 2 \\
31 / 2 \\
21 / 2 \\
11 / 2\end{array}$ & $\begin{array}{l}44288.76 \\
44523.45 \\
44696.29 \\
44814.73\end{array}$ & $\begin{array}{l}-234.69 \\
-172.84 \\
-118.44\end{array}$ & $\begin{array}{l}\text { 1. } 317 \\
\text { 1. } 240 \\
\text { 1. } 030 \\
0.400\end{array}$ \\
\hline $3 d^{6}\left(a^{5} \mathrm{D}\right) 4 p$ & $x^{6} \mathrm{P}^{\circ}$ & $\begin{array}{l}31 / 2 \\
21 / 2 \\
11 / 2\end{array}$ & $\begin{array}{l}\text { 44993. } 92 \\
45156.11 \\
45259.17\end{array}$ & $\begin{array}{l}-162.19 \\
-103.06\end{array}$ & $\begin{array}{l}\text { 1. } 717 \\
\text { 1. } 885 \\
\text { 2. } 399\end{array}$ \\
\hline $3 d^{6}\left(a^{5} \mathrm{D}\right) 4 p$ & $z^{4} \mathrm{D}^{\circ}$ & $\begin{array}{l}31 / 2 \\
21 / 2 \\
11 / 2 \\
01 / 2\end{array}$ & $\begin{array}{l}45754.27 \\
45940.93 \\
46083.89 \\
46169.93\end{array}$ & $\begin{array}{r}-186.66 \\
-142.96 \\
-86.04\end{array}$ & $\begin{array}{l}\text { 1. } 427 \\
\text { 1. } 372 \\
\text { 1. } 200 \\
0.000\end{array}$ \\
\hline $3 d^{5} 4 s\left(a^{7} \mathrm{~S}\right) 5 p$ & $y^{8} \mathrm{P}^{\circ}$ & $\begin{array}{l}21 / 2 \\
31 / 2 \\
41 / 2\end{array}$ & $\begin{array}{l}45981.44 \\
46000.77 \\
46026.75\end{array}$ & $\begin{array}{l}\text { 19. } 33 \\
25.98\end{array}$ & \\
\hline $3 d^{6}\left(a^{5} \mathrm{D}\right) 4 p$ & $y{ }^{4} \mathrm{P}^{\circ}$ & $\begin{array}{l}21 / 2 \\
11 / 2 \\
01 / 2\end{array}$ & $\begin{array}{l}46901.13 \\
47154.51 \\
47299.29\end{array}$ & $\begin{array}{l}-253.38 \\
-144.78\end{array}$ & $\begin{array}{l}\text { 1. } 595 \\
\text { 1. } 732 \\
\text { 2. } 666\end{array}$ \\
\hline $3 d^{5} 4 s\left(a^{7} \mathrm{~S}\right) 5 p$ & $w^{6} \mathrm{P}^{\circ}$ & $\begin{array}{l}31 / 2 \\
21 / 2 \\
11 / 2\end{array}$ & $\begin{array}{l}47387.62 \\
47659.52 \\
47782.43\end{array}$ & $\begin{array}{l}-271.90 \\
-122.91\end{array}$ & $\begin{array}{l}\text { 1. } 713 \\
\text { 1. } 952 \\
\text { 2. } 666\end{array}$ \\
\hline $3 d^{5} 4 s\left(a^{5} \mathrm{P}\right) 4 p$ & $y{ }^{6} \mathrm{D}^{\circ}$ & $\begin{array}{l}01 / 2 \\
11 / 2 \\
21 / 2 \\
31 / 2 \\
41 / 2\end{array}$ & $\begin{array}{l}47452.16 \\
47466.66 \\
47753.99 \\
47774.52 \\
47903.80\end{array}$ & $\begin{array}{r}14.50 \\
287.33 \\
20.53 \\
129.28\end{array}$ & $\begin{array}{l}3.174 \\
1.820 \\
\text { 1. } 594 \\
1.540\end{array}$ \\
\hline $3 d^{5} 4 s\left(a^{5} \mathrm{G}\right) 4 p$ & $y{ }^{6} \mathrm{~F}^{\circ}$ & $\begin{array}{l}51 / 2 \\
41 / 2 \\
31 / 2 \\
21 / 2 \\
11 / 2 \\
01 / 2\end{array}$ & $\begin{array}{l}48021.43 \\
48168.01 \\
48225.99 \\
48270.91 \\
48300.98 \\
48318.12\end{array}$ & $\begin{array}{r}-146.58 \\
-57.98 \\
-44.92 \\
-30.07 \\
-17.14\end{array}$ & $\begin{array}{r}\text { 1. } 460 \\
\text { 1. } 432 \\
\text { 1. } 043 \\
\text { 1. } 319 \\
\text { 1. } 068 \\
-0.496\end{array}$ \\
\hline
\end{tabular}


Table 2. Odd Terms of Mn I-Continued

\begin{tabular}{|c|c|c|c|c|c|}
\hline Config. & Desig. & $J$ & Level & Interval & Obs. g \\
\hline $3 d^{5} 4 s\left(a^{5} \mathrm{P}\right) 4 p$ & $v^{6} \mathrm{P}^{\circ}$ & $\begin{array}{l}31 / 2 \\
21 / 2 \\
11 / 2\end{array}$ & $\begin{array}{l}49888.08 \\
50012.53 \\
50099.16\end{array}$ & $\begin{array}{r}-124.45 \\
-86.63\end{array}$ & $\begin{array}{l}\text { 1. } 711 \\
\text { 1. } 888 \\
\text { 2. } 398\end{array}$ \\
\hline $3 d^{5} 4 s\left(a^{5} \mathrm{G}\right) 4 p$ & $z^{4} \mathrm{H}^{\circ}$ & $\begin{array}{l}31 / 2 \\
41 / 2 \\
51 / 2 \\
61 / 2\end{array}$ & $\begin{array}{l}50065.46 \\
50072.59 \\
50081.31 \\
50094.60\end{array}$ & $\begin{array}{r}\text { 7. } 13 \\
\text { 8. } 72 \\
\text { 13. } 29\end{array}$ & 1. 22 \\
\hline $3 d^{5} 4 s\left(a^{5} \mathrm{G}\right) 4 p$ & $y^{4} \mathrm{~F}^{\circ}$ & $\begin{array}{l}41 / 2 \\
31 / 2 \\
21 / 2 \\
11 / 2\end{array}$ & $\begin{array}{l}50341.30 \\
50359.28 \\
50373.23 \\
50383.27\end{array}$ & $\begin{array}{l}-17.98 \\
-13.95 \\
-10.04\end{array}$ & $\begin{array}{l}\text { 1. } 318 \\
\text { 1. } 242 \\
\text { 1. } 03\end{array}$ \\
\hline $3 d^{5} 4 s\left(b{ }^{5} \mathrm{D}\right) 4 p$ & $x^{6} \mathrm{~F}^{\circ}$ & $\begin{array}{l}01 / 2 \\
11 / 2 \\
21 / 2 \\
31 / 2 \\
41 / 2 \\
51 / 2\end{array}$ & $\begin{array}{l}50818.64 \\
50863.05 \\
50931.42 \\
51014.95 \\
51100.49 \\
51169.18\end{array}$ & $\begin{array}{l}44.41 \\
68.37 \\
83.53 \\
85.54 \\
68.69\end{array}$ & $\begin{array}{r}-0.62 \\
\text { 1. } 07 \\
\text { 1. } 316\end{array}$ \\
\hline $3 d^{5} 4 s\left(a{ }^{5} \mathrm{P}\right) 4 p$ & $x{ }^{4} \mathrm{P}^{\circ}$ & $\begin{array}{l}21 / 2 \\
11 / 2 \\
01 / 2\end{array}$ & $\begin{array}{l}51305.41 \\
51445.65 \\
51552.78\end{array}$ & $\begin{array}{l}-140.24 \\
-107.13\end{array}$ & $\begin{array}{l}\text { 1. } 591 \\
\text { 1. } 728 \\
\text { 2. } 664\end{array}$ \\
\hline $3 d^{5} 4 s\left(a{ }^{5} \mathrm{G}\right) 4 p$ & $z^{4} \mathrm{G}^{\circ}$ & $\begin{array}{l}21 / 2 \\
31 / 2 \\
41 / 2 \\
51 / 2\end{array}$ & $\begin{array}{l}51515.63 \\
51530.61 \\
51546.27 \\
51560.93\end{array}$ & $\begin{array}{l}\text { 14. } 98 \\
\text { 15. } 66 \\
\text { 14. } 66\end{array}$ & 1. 273 \\
\hline $3 d^{5} 4 s\left(b{ }^{5} \mathrm{D}\right) 4 p$ & $u{ }^{6} \mathrm{P}^{\circ}$ & $\begin{array}{l}11 / 2 \\
21 / 2 \\
31 / 2\end{array}$ & $\begin{array}{l}52015.00 \\
52128.65 \\
52253.24\end{array}$ & $\begin{array}{l}113.65 \\
124.59\end{array}$ & 1. 71 \\
\hline $3 d^{5} 4 s\left(b{ }^{5} \mathrm{D}\right) 4 p$ & $x{ }^{6} \mathrm{D}^{\circ}$ & $\begin{array}{l}41 / 2 \\
31 / 2 \\
21 / 2 \\
11 / 2 \\
01 / 2\end{array}$ & $\begin{array}{l}52758.11 \\
52870.10 \\
52883.87 \\
52883.87 \\
52883.10\end{array}$ & $\begin{array}{r}-111.99 \\
-13.77 \\
0.00 \\
0.77\end{array}$ & $\begin{array}{l}\text { 1. } 552 \\
\text { 1. } 57\end{array}$ \\
\hline $3 d^{5} 4 s\left(a^{7} \mathbf{S}\right) 4 f$ & $z^{8} \mathrm{~F}^{\circ}$ & $\begin{array}{c}01 / 2 \\
\text { to } \\
61 / 2\end{array}$ & $52974.5 ?$ & & \\
\hline $3 d^{5} 4 s\left(a^{7} \mathbf{S}\right) 4 f$ & $w^{6} \mathrm{~F}^{\circ}$ & $\begin{array}{l}01 / 2 \\
11 / 2 \\
21 / 2 \\
31 / 2 \\
41 / 2 \\
51 / 2\end{array}$ & $\begin{array}{l}52977.93 \\
52978.03 \\
52977.82 \\
52977.75 \\
52977.89\end{array}$ & $\begin{array}{r}0.10 \\
-0.21 \\
-0.07 \\
0.14\end{array}$ & \\
\hline $3 d^{5} 4 s\left(a^{5} \mathrm{P}\right) 4 p$ & $y{ }^{4} \mathrm{D}^{\circ}$ & $\begin{array}{l}01 / 2 \\
11 / 2 \\
21 / 2 \\
31 / 2\end{array}$ & $\begin{array}{l}53101.32 \\
53103.19 \\
53109.21 \\
53124.09\end{array}$ & $\begin{array}{r}\text { 1. } 87 \\
6.02 \\
14.88\end{array}$ & 1. 423 \\
\hline $3 d^{5} 4 s\left(a^{7} \mathrm{~S}\right) 6 p$ & $t^{6} \mathrm{P}^{\circ}$ & $\begin{array}{l}31 / 2 \\
21 / 2 \\
11 / 2\end{array}$ & $\begin{array}{l}53261.42 \\
53291.58 \\
53311.37\end{array}$ & $\begin{array}{l}-30.16 \\
-19.79\end{array}$ & \\
\hline $3 d^{5} 4 s\left(a^{5} \mathrm{P}\right) 4 p$ & $z^{4} \mathrm{~S}^{\circ}$ & $11 / 2$ & 54218. 71 & & 1. 770 \\
\hline $3 d^{5} 4 s\left(b{ }^{5} \mathrm{D}\right) 4 p$ & $x^{4} \mathrm{D}^{\circ}$ & $\begin{array}{l}31 / 2 \\
21 / 2 \\
11 / 2 \\
01 / 2\end{array}$ & $\begin{array}{l}55107.52 \\
55186.17 \\
55279.91\end{array}$ & $\begin{array}{l}-78.65 \\
-93.74\end{array}$ & $\begin{array}{l}\text { 1. } 407 \\
\text { 1. } 365 \\
0.826\end{array}$ \\
\hline $3 d^{5} 4 s\left(b{ }^{5} \mathrm{D}\right) 4 p$ & $w^{4} \mathrm{P}^{\circ}$ & $\begin{array}{l}21 / 2 \\
11 / 2 \\
01 / 2\end{array}$ & $\begin{array}{l}55405.14 \\
55368.66 \\
55457.20\end{array}$ & $\begin{array}{r}36.48 \\
-88.54\end{array}$ & 2. 28 \\
\hline
\end{tabular}


Table 2. Odd Terms of Mn I-Continued

\begin{tabular}{|c|c|c|c|c|c|}
\hline Config. & Desig. & $J$ & Level & Interval & Obs. $\mathrm{g}$ \\
\hline $3 d^{5} 4 s\left(a^{7} \mathrm{~S}\right) 5 f$ & $v^{6} \mathrm{~F}^{\circ}$ & $\begin{array}{l}01 / 2 \\
11 / 2 \\
21 / 2 \\
31 / 2 \\
41 / 2 \\
51 / 2\end{array}$ & $\begin{array}{l}55491.95 \\
55491.57 \\
55492.08 \\
55492.52 \\
55492.74\end{array}$ & $\begin{array}{r}-0.38 \\
0.15 \\
0.44 \\
0.22\end{array}$ & \\
\hline $3 d^{5} 4 s\left(a^{7} \mathrm{~S}\right) 5 f$ & $y^{8} \mathrm{~F}^{\circ}$ & $\begin{array}{l}61 / 2 \\
51 / 2 \\
41 / 2 \\
31 / 2 \\
21 / 2 \\
11 / 2 \\
01 / 2\end{array}$ & $\left\{\begin{array}{l}55498.5 \\
55499.09 \\
55499.09 \\
55499.5 \\
55499.90 \\
55499.75\end{array}\right.$ & $\begin{array}{r}-0.6 \\
0.0 \\
-0.4 \\
-0.4 \\
0.15\end{array}$ & \\
\hline & & $21 / 2$ & 55923. 81 & & \\
\hline $3 d^{5} 4 s\left(a^{5} \mathrm{~S}\right) 5 p$ & $s^{6} \mathrm{P}^{\circ} ?$ & $\begin{array}{l}11 / 2 \\
21 / 2 \\
31 / 2\end{array}$ & $\begin{array}{l}55996.62 \\
56007.91 \\
56012.42\end{array}$ & $\begin{array}{r}\text { 11. } 29 \\
\text { 4. } 51\end{array}$ & \\
\hline $3 d^{5} 4 s\left(a^{7} \mathrm{~S}\right) 6 f$ & $u^{6} \mathrm{~F}^{\circ}$ & $\begin{array}{c}01 / 2 \\
\text { to } \\
61 / 2\end{array}$ & 56867.1 & & \\
\hline $3 d^{5} 4 s\left(b^{3} \mathrm{P}\right) 4 p$ & $v^{4} \mathrm{P}^{\circ}$ & $\begin{array}{l}01 / 2 \\
11 / 2 \\
21 / 2\end{array}$ & $\begin{array}{l}57228.30 \\
57360.78 \\
57487.08\end{array}$ & $\begin{array}{l}132.48 \\
126.30\end{array}$ & $\begin{array}{l}\text { 2. } 671 \\
\text { 1. } 736 \\
\text { 1. } 590\end{array}$ \\
\hline $3 d^{5} 4 s\left(b^{3} \mathrm{P}\right) 4 p$ & $y^{4} \mathrm{~S}^{\circ}$ & $11 / 2$ & 57512.16 & & 2. 000 \\
\hline $3 d^{5} 4 s\left(a^{7} \mathrm{~S}\right) 7 f$ & $t^{6} \mathrm{~F}^{\circ}$ & $\begin{array}{l}01 / 2 \\
\text { to } \\
61 / 2\end{array}$ & 57697.2 & & \\
\hline $3 d^{6}\left(a^{3} \mathrm{H}\right) 4 p$ & $y{ }^{4} \mathrm{G}^{\circ}$ & $\begin{array}{l}51 / 2 \\
41 / 2 \\
31 / 2 \\
21 / 2\end{array}$ & $\begin{array}{l}58075.06 \\
58110.24 \\
58136.69 \\
58159.73\end{array}$ & $\begin{array}{l}-35.18 \\
-26.45 \\
-23.04\end{array}$ & $\begin{array}{l}\text { 1. } 269 \\
\text { 1. } 168 \\
0.980 \\
0.578\end{array}$ \\
\hline $3 d^{6}\left(a^{3} \mathrm{H}\right) 4 p$ & $y{ }^{4} \mathrm{H}^{\circ}$ & $\begin{array}{l}61 / 2 \\
51 / 2 \\
41 / 2 \\
31 / 2\end{array}$ & $\begin{array}{l}58338.67 \\
58427.30 \\
58485.52 \\
58519.90\end{array}$ & $\begin{array}{l}-88.63 \\
-58.22 \\
-34.38\end{array}$ & $\begin{array}{l}\text { 1. } 228 \\
\text { 1. } 133 \\
0.968 \\
0.665\end{array}$ \\
\hline $3 d^{6}\left(a^{3} \mathrm{H}\right) 4 p$ & $z^{4} \mathrm{I}^{\circ}$ & $\begin{array}{l}71 / 2 \\
61 / 2 \\
51 / 2 \\
41 / 2\end{array}$ & $\begin{array}{l}58852.60 \\
58843.39 \\
58851.49 \\
58866.66\end{array}$ & $\begin{array}{r}9.21 \\
-8.10 \\
-15.17\end{array}$ & $\begin{array}{l}\text { 1. } 09 \\
\text { 0. } 73\end{array}$ \\
\hline $3 d^{6}\left(a^{3} \mathrm{P}\right) 4 p$ & $u{ }^{4} \mathrm{P}^{\circ}$ & $\begin{array}{l}21 / 2 \\
11 / 2 \\
01 / 2\end{array}$ & $\begin{array}{l}59116.60 \\
59384.45 \\
59568.29\end{array}$ & $\begin{array}{l}-267.85 \\
-183.84\end{array}$ & $\begin{array}{l}\text { 1. } 558 \\
\text { 1. } 608 \\
\text { 1. } 94\end{array}$ \\
\hline $3 d^{6}\left(a^{3} \mathrm{~F}\right) 4 p$ & $x^{4} \mathrm{~F}^{\circ}$ & $\begin{array}{l}41 / 2 \\
31 / 2 \\
21 / 2 \\
11 / 2\end{array}$ & $\begin{array}{l}59257.44 \\
59290.23 \\
59360.72 \\
59416.15\end{array}$ & $\begin{array}{r}-32.79 \\
-70.49 \\
-55.43\end{array}$ & $\begin{array}{l}\text { 1. } 327 \\
\text { 1. } 325 \\
\text { 1. } 11 \\
0.39\end{array}$ \\
\hline $3 d^{6}\left(a^{3} \mathrm{P}\right) 4 p$ & $w^{4} \mathrm{D}^{\circ}$ & $\begin{array}{l}31 / 2 \\
21 / 2 \\
11 / 2 \\
01 / 2\end{array}$ & $\begin{array}{l}59339.55 \\
59600.35 \\
59989.77 \\
60141.98\end{array}$ & $\begin{array}{l}-260.86 \\
-389.42 \\
-152.21\end{array}$ & $\begin{array}{l}\text { 1. } 362 \\
\text { 1. } 277 \\
\text { 1. } 194 \\
0.17\end{array}$ \\
\hline $3 d^{6}\left(a^{3} \mathrm{~F}\right) 4 p$ & $v^{4} \mathrm{D}^{\circ}$ & $\begin{array}{l}31 / 2 \\
21 / 2 \\
11 / 2 \\
01 / 2\end{array}$ & $\begin{array}{l}59470.14 \\
59480.80 \\
59527.89 \\
59527.36\end{array}$ & $\begin{array}{r}-10.66 \\
-47.09 \\
0.53\end{array}$ & $\begin{array}{l}\text { 1. } 386 \\
\text { 1. } 281\end{array}$ \\
\hline $3 d^{6}\left(a^{3} \mathrm{H}\right) 4 p$ & $z^{2} \mathrm{I}^{\circ}$ & $\begin{array}{l}61 / 2 \\
51 / 2\end{array}$ & $\begin{array}{l}59617.12 \\
59827.88\end{array}$ & -210.76 & $\begin{array}{l}\text { 1. } 074 \\
0.93\end{array}$ \\
\hline
\end{tabular}


Table 2. Odd Terms of Mn I-Continued

\begin{tabular}{|c|c|c|c|c|c|}
\hline Config. & Desig. & $J$ & Level & Interval & Obs. g \\
\hline $3 d^{6}\left(a^{3} \mathrm{~F}\right) 4 p$ & $x{ }^{4} \mathrm{G}^{\circ}$ & $\begin{array}{l}51 / 2 \\
41 / 2 \\
31 / 2 \\
21 / 2\end{array}$ & $\begin{array}{l}59652.90 \\
59731.94 \\
59784.31 \\
59817.70\end{array}$ & $\begin{array}{l}-79.04 \\
-52.37 \\
-33.39\end{array}$ & $\begin{array}{l}\text { 1. } 239 \\
\text { 1. } 169 \\
0.990 \\
0.584\end{array}$ \\
\hline $\operatorname{Mn} I I\left({ }^{7} S_{3}\right)$ & Limit & $\ldots$ & 59970 & & \\
\hline $3 d^{6}\left({ }^{3} \mathrm{P}\right) 4 p$ & $z^{2} \mathrm{D}^{\circ}$ & $\begin{array}{l}21 / 2 \\
11 / 2\end{array}$ & $\begin{array}{l}60101.65 \\
60395.64\end{array}$ & -293.99 & $\begin{array}{l}\text { 1. } 31 \\
0.91\end{array}$ \\
\hline \multirow[t]{2}{*}{$3 d^{6}\left(a^{3} \mathrm{H}\right) 4 p$} & $z^{2} \mathrm{G}^{\circ}$ & $\begin{array}{l}41 / 2 \\
31 / 2\end{array}$ & $\begin{array}{l}60668.49 \\
60739.42\end{array}$ & -70.93 & 1. 112 \\
\hline & $w^{4} \mathrm{~F}^{\circ}$ & $\begin{array}{l}11 / 2 \\
21 / 2 \\
31 / 2 \\
41 / 2\end{array}$ & $\begin{array}{l}60760.87 \\
60820.35 \\
60902.80 \\
60938.97\end{array}$ & $\begin{array}{l}59.48 \\
82.45 \\
36.17\end{array}$ & \\
\hline $3 d^{5} 4 s\left({ }^{3} \mathbf{I}\right) 4 p$ & $x{ }^{4} \mathrm{H}^{\circ}$ & $\begin{array}{l}61 / 2 \\
51 / 2 \\
41 / 2 \\
31 / 2\end{array}$ & $\begin{array}{l}60891.48 \\
60933.73 \\
60955.88 \\
60957.21\end{array}$ & $\begin{array}{r}-42.25 \\
-22.15 \\
-1.33\end{array}$ & $\begin{array}{l}\text { 1. } 228 \\
\text { 1. } 134\end{array}$ \\
\hline \multirow[t]{7}{*}{$3 d^{5} 4 s\left({ }^{3} \mathbf{I}\right) 4 p$} & $y^{4} \mathrm{I}^{\circ}$ & $\begin{array}{l}71 / 2 \\
61 / 2 \\
51 / 2 \\
41 / 2\end{array}$ & $\begin{array}{l}61204.54 \\
61225.55 \\
61225.77 \\
61211.43\end{array}$ & $\begin{array}{r}-21.01 \\
-0.22 \\
14.34\end{array}$ & $\begin{array}{l}\text { 1. } 20 \\
\text { 0. } 75\end{array}$ \\
\hline & $w^{4} \mathrm{G}^{\circ}$ & $\begin{array}{l}51 / 2 \\
41 / 2 \\
31 / 2 \\
21 / 2\end{array}$ & $\begin{array}{l}61469.21 \\
61485.34 \\
61480.60 \\
61471.23\end{array}$ & $\begin{array}{r}-16.13 \\
4.74 \\
9.37\end{array}$ & $\begin{array}{l}\text { 1. } 164 \\
\text { 1. } 020 \\
\text { 1. } 13\end{array}$ \\
\hline & $z^{2} \mathrm{~F}^{\circ}$ & $\begin{array}{l}31 / 2 \\
21 / 2\end{array}$ & $\begin{array}{l}61710.98 \\
61727.46\end{array}$ & -16.48 & \\
\hline & $y^{2} \mathrm{G}^{\circ}$ & $\begin{array}{l}41 / 2 \\
31 / 2\end{array}$ & $\begin{array}{l}61714.52 \\
61785.94\end{array}$ & -71.42 & 0. 93 \\
\hline & & $51 / 2$ & 61744.04 & & \\
\hline & $y^{2} \mathrm{I}^{\circ}$ & $\begin{array}{l}51 / 2 \\
61 / 2\end{array}$ & $\begin{array}{l}61819.07 \\
61912.57\end{array}$ & 93.50 & \\
\hline & $y^{2} \mathrm{~F}^{\circ}$ & $\begin{array}{l}31 / 2 \\
21 / 2\end{array}$ & $\begin{array}{l}62034.04 \\
62075.02\end{array}$ & -40.98 & 0.58 \\
\hline $3 d^{6}\left({ }^{3} \mathrm{P}\right) 4 p$ & $z^{2} \mathrm{P}^{\circ}$ & $\begin{array}{l}11 / 2 \\
01 / 2\end{array}$ & $\begin{array}{l}62354.76 \\
62391.05\end{array}$ & -36.29 & $\begin{array}{l}\text { 1. } 24 \\
0.81\end{array}$ \\
\hline $3 d^{6}\left(a^{3} \mathrm{G}\right) 4 p$ & $v^{4} \mathrm{~F}^{\circ}$ & $\begin{array}{l}11 / 2 \\
21 / 2 \\
31 / 2 \\
41 / 2\end{array}$ & $\begin{array}{l}62390.20 \\
62487.36 \\
62505.29 \\
62392.82\end{array}$ & $\begin{array}{r}97.16 \\
17.93 \\
-112.47\end{array}$ & 1. 35 \\
\hline \multirow[t]{3}{*}{$3 d^{5} 4 s\left(a^{5} \mathrm{~F}\right) 4 p$} & $w^{6} \mathrm{D}^{\circ}$ & $\begin{array}{l}41 / 2 \\
31 / 2 \\
21 / 2 \\
11 / 2 \\
01 / 2\end{array}$ & $\begin{array}{l}62670.81 \\
62851.47 \\
62761.33 \\
62787.63 \\
62768.16\end{array}$ & $\begin{array}{r}-180.66 \\
90.14 \\
-26.30 \\
19.47\end{array}$ & \\
\hline & $y{ }^{2} \mathrm{D}^{\circ}$ & $\begin{array}{l}21 / 2 \\
11 / 2\end{array}$ & $\begin{array}{l}\text { 63081. } 28 \\
63114.11\end{array}$ & -32.83 & $\begin{array}{l}\text { 1. } 24 \\
0.758\end{array}$ \\
\hline & $x^{2} \mathrm{~F}^{\circ}$ & $\begin{array}{l}21 / 2 \\
31 / 2\end{array}$ & $\begin{array}{l}\text { 63139. } 70 \\
63288.78\end{array}$ & 149. 08 & \\
\hline $3 d^{6}\left({ }^{3} \mathrm{H} ?\right) 4 p$ & $z^{2} \mathrm{H}^{\circ}$ & $\begin{array}{l}51 / 2 \\
41 / 2\end{array}$ & $\begin{array}{l}63288.78 \\
63548.49\end{array}$ & -259.71 & $\begin{array}{l}\text { 1. } 127 \\
0.92\end{array}$ \\
\hline
\end{tabular}


Table 2. Odd Terms of Mn I-Continued

\begin{tabular}{|c|c|c|c|c|c|}
\hline Config. & Desig. & $J$ & Level & Interval & Obs. g \\
\hline \multirow{21}{*}{$3 d^{6}\left({ }^{3} \mathrm{G}\right) 4 p$} & $y^{2} \mathrm{H}^{\circ}$ & $\begin{array}{l}31 / 2 \\
41 / 2 \\
51 / 2\end{array}$ & $\begin{array}{l}63319.85 ? \\
63347.91 \\
63449.13\end{array}$ & 101. 22 & \\
\hline & $w^{4} \mathrm{H}^{\circ}$ & $\begin{array}{l}61 / 2 \\
51 / 2 \\
41 / 2 \\
31 / 2\end{array}$ & $\begin{array}{l}63363.54 \\
63457.85 \\
63444.61 \\
63395.45\end{array}$ & $\begin{array}{r}-94.31 \\
13.24 \\
49.16\end{array}$ & $\begin{array}{l}\text { 1. } 231 \\
\text { 1. } 14 \\
0.70\end{array}$ \\
\hline & & $21 / 2$ & 63371.56 & & \\
\hline & & $41 / 2$ & 63374.53 & & \\
\hline & & $21 / 2$ & 63523. 82 & & \\
\hline & & $31 / 2$ & 63546. 30 & & \\
\hline & & $11 / 2,21 / 2$ & 63583.84 & & \\
\hline & $x^{2} \mathrm{D}^{\circ}$ & $\begin{array}{l}21 / 2 \\
11 / 2\end{array}$ & $\begin{array}{l}63764.90 \\
63845.32\end{array}$ & -80.42 & \\
\hline & $x^{2} \mathrm{H}^{\circ}$ & $\begin{array}{l}51 / 2 \\
41 / 2\end{array}$ & $\begin{array}{l}\text { 64051. } 91 \\
64055.37\end{array}$ & -3.46 & \\
\hline & $u^{4} \mathrm{D}^{\circ}$ & $\begin{array}{l}31 / 2 \\
21 / 2 \\
11 / 2 \\
01 / 2\end{array}$ & $\begin{array}{l}64409.69 \\
64712.94 \\
64683.95 \\
64638.68\end{array}$ & $\begin{array}{r}-303.25 \\
28.99 \\
45.27\end{array}$ & $\begin{array}{l}\text { 1. } 42 \\
\text { 1. } 22 \\
0.22\end{array}$ \\
\hline & $x^{2} \mathrm{G}^{\circ}$ & $\begin{array}{l}41 / 2 \\
31 / 2\end{array}$ & $\begin{array}{l}\text { 64585. } 44 \\
64649.20\end{array}$ & -63.76 & 1. 307 \\
\hline & $v^{4} \mathrm{H}^{\circ}$ & $\begin{array}{l}61 / 2 \\
51 / 2 \\
41 / 2 \\
31 / 2\end{array}$ & $\begin{array}{l}64731.88 \\
64819.53 \\
64888.00 \\
64920.33\end{array}$ & $\begin{array}{l}-87.65 \\
-68.47 \\
-32.33\end{array}$ & $\begin{array}{l}\text { 1. } 236 \\
\text { 1. } 137 \\
\text { 0. } 974\end{array}$ \\
\hline & $w^{2} \mathrm{~F}^{\circ}$ & $\begin{array}{l}21 / 2 \\
31 / 2\end{array}$ & $\begin{array}{l}\text { 64823. } 21 \\
64988.22\end{array}$ & 165. 01 & \\
\hline & $w^{2} \mathrm{G}^{\circ}$ & $\begin{array}{l}41 / 2 \\
31 / 2\end{array}$ & $\begin{array}{l}\text { 65262. } 28 \\
\text { 65305. } 13\end{array}$ & -42.85 & 1. 13 \\
\hline & $v^{2} \mathrm{~F}^{\circ}$ & $\begin{array}{l}31 / 2 \\
21 / 2\end{array}$ & $\begin{array}{l}\text { 65617. } 37 \\
65649.13 ?\end{array}$ & -31.76 & 1. 015 \\
\hline & & $41 / 2$ & 65768.81 & & 1. 12 \\
\hline & $v^{4} \mathrm{G}^{\circ}$ & $\begin{array}{l}21 / 2 \\
31 / 2 \\
41 / 2 \\
51 / 2\end{array}$ & $\begin{array}{l}65873.40 \\
65876.34 \\
65908.92 \\
65887.31\end{array}$ & $\begin{array}{r}2.94 \\
32.58 \\
-21.61\end{array}$ & $\begin{array}{l}\text { 1. } 160 \\
\text { 1. } 259\end{array}$ \\
\hline & $w^{2} \mathrm{D}^{\circ}$ & $\begin{array}{l}21 / 2 \\
11 / 2\end{array}$ & $\begin{array}{l}65946.87 \\
65961.90\end{array}$ & -15.03 & 1. 30 \\
\hline & $u^{2} \mathrm{~F}^{\circ}$ & $\begin{array}{l}21 / 2 \\
31 / 2\end{array}$ & $\begin{array}{l}66020.63 \\
66149.10\end{array}$ & 128. 47 & 1. 14 \\
\hline & $u{ }^{4} \mathrm{H}^{\circ}$ & $\begin{array}{l}31 / 2 \\
41 / 2 \\
51 / 2 \\
61 / 2\end{array}$ & $\begin{array}{l}66334.47 \\
66356.40 \\
66418.55 \\
66568.58\end{array}$ & $\begin{array}{r}21.93 \\
62.15 \\
150.03\end{array}$ & $\begin{array}{l}\text { 0. } 764 \\
\text { 1. } 022 \\
\\
\text { 1. } 23\end{array}$ \\
\hline & $u{ }^{4} \mathrm{G}^{\circ}$ & $\begin{array}{l}21 / 2 \\
31 / 2 \\
41 / 2 \\
51 / 2\end{array}$ & $\begin{array}{l}66395.19 \\
66454.27 \\
66522.62 \\
66573.60\end{array}$ & $\begin{array}{l}59.08 \\
68.35 \\
50.98\end{array}$ & $\begin{array}{l}0.611 \\
0.932 \\
\text { 1. } 13 \\
\text { 1. } 24\end{array}$ \\
\hline
\end{tabular}


Table 2. Odd Terms of Mn I-Continued

\begin{tabular}{|c|c|c|c|c|c|}
\hline Config. & Desig. & $J$ & Level & Interval & Obs. g \\
\hline & & $11 / 2$ & 66504.21 & & \\
\hline & & $31 / 2$ & 66600.17 & & \\
\hline & $v^{2} \mathrm{G}^{\circ}$ & $\begin{array}{l}41 / 2 \\
31 / 2\end{array}$ & $\begin{array}{l}66630.92 \\
66737.82\end{array}$ & -106.90 & $\begin{array}{l}\text { 1. } 13 \\
0.46\end{array}$ \\
\hline & & $21 / 2$ & $66654.65 ?$ & & \\
\hline & $u^{4} \mathrm{~F}^{\circ}$ & $\begin{array}{l}11 / 2 \\
21 / 2 \\
31 / 2 \\
41 / 2\end{array}$ & $\begin{array}{l}66843.79 \\
66837.64 \\
66783.05 \\
66855.00\end{array}$ & $\begin{array}{r}-6.15 \\
-54.59 \\
71.95\end{array}$ & $\begin{array}{l}0.46 \\
\\
\text { 1. } 21 \\
1.33\end{array}$ \\
\hline & & $2 \frac{1}{2} ?$ & 66910.02 & & \\
\hline & & $31 / 2$ & 66981. 30 & & 1. 33 \\
\hline & & $21 / 2$ & 67008.54 & & \\
\hline & $w^{2} \mathrm{H}^{\circ}$ & $\begin{array}{l}51 / 2 \\
41 / 2\end{array}$ & $\begin{array}{l}67504.90 \\
67576.84\end{array}$ & -71.94 & $\begin{array}{l}1.09 \\
0.90\end{array}$ \\
\hline & $t^{4} \mathrm{G}^{\circ}$ & $\begin{array}{l}51 / 2 \\
41 / 2 \\
31 / 2 \\
21 / 2\end{array}$ & $\begin{array}{l}67752.84 \\
67819.17 \\
67891.36 \\
67964.87\end{array}$ & $\begin{array}{l}-66.33 \\
-72.19 \\
-73.51\end{array}$ & 1. 266 \\
\hline & $u^{2} \mathrm{G}^{\circ}$ & $\begin{array}{l}41 / 2 \\
31 / 2\end{array}$ & $\begin{array}{l}68286.44 \\
68338.59\end{array}$ & -52.15 & 1. 320 \\
\hline $3 d^{6}\left({ }^{1} \mathrm{I}\right) 4 p$ & $z^{2} \mathrm{~K}^{\circ}$ & $\begin{array}{l}71 / 2 \\
61 / 2\end{array}$ & $\begin{array}{l}68797.56 ? \\
68842.52 ?\end{array}$ & -44.96 & $\begin{array}{l}\text { 1. } 07 \\
0.93\end{array}$ \\
\hline $3 d^{6}\left({ }^{1} \mathrm{I}\right) 4 p$ & $x^{2} I^{\circ}$ & $\begin{array}{l}61 / 2 \\
51 / 2\end{array}$ & $\begin{array}{l}69560.88 ? \\
69629.85 ?\end{array}$ & -68.97 & $\begin{array}{l}\text { 1. } 07 \\
0.924\end{array}$ \\
\hline $3 d^{6}\left({ }^{1} \mathrm{I}\right) 4 p ?$ & $v^{2} \mathrm{H}^{\circ}$ & $\begin{array}{l}41 / 2 \\
51 / 2\end{array}$ & $\begin{array}{l}\text { 69663. } 20 ? \\
69722.96\end{array}$ & 59. 76 & 1. 10 \\
\hline
\end{tabular}

the average differences do not exceed $0.03 \mathrm{~K}$, we have retained the older values.

In col. 2, some intensity numbers are accompanied by literal symbols for line character; these have the following meanings, $c=$ complex,$d=$ double, $h=$ hazy, $H=$ very hazy, $l=$ shaded long ward, $r=$ narrow selfreversal, $R=$ wide self-reversal, $s=$ shaded short ward, $w=$ wide. Excepting self-reversals, the remaining symbols for line character frequently suggest unresolved hyperfine structure because the manganese nucleus has a spin of $5 / 2(h / 2 \pi)$ and a magnetic moment of 3.5 nuclear magnetons.

Table 1 contains 2030 classified lines of Mn I, including 55 accepted double classifications for lack of adequate spectroscopic resolving power. The energy levels derived from these classified levels are symbolized in col. 5, and the difference between their numerical values and the vacuum wave number associated with the measured wavelength is shown in col. 4, $\mathrm{O}-\mathrm{C}$. The average $\mathrm{O}-\mathrm{C}$ for all classified lines is $0.06 \mathrm{~K}$.

The electron configurations, numerical values, and $g$-factors associated with the atomic-energy-level symbols in col. 5 of table 1 , will be found in table 2 .
In the last column of table 1 appear Zeeman-type numbers for 390 classified lines of $\mathrm{Mn}$ I according to the description of basic types of Zeeman patterns by Back and Landé $[32,34$.] Briefly, types $4,5,6$ are restricted to even multiplicities (doublets, quartets, sextets, octets) whose energy levels always have half-integral inner-quantum number ( $J$-values). In type 4 , the level with larger $J$ has the smaller $g$, in type 5 the level with larger $J$ also has the larger $g$ and in type 6 , the two combining levels have equal $J$ but unequal $g$ values. Type $7 \mathrm{~b}$ is a special case of types 4, 5, 6 in which the $g$ values of both combining levels are equal so that the observed pattern is a pseudo triplet with one $p$ component and two $n$ components. In the case of equal $J$ and equal $g$, the type is represented by $6,7 \mathrm{~b}$, and the displacement of the $n$ components in Lorentz units expresses both $g$ values.

The letter $\mathrm{C}$ attached to a dozen type numbers in col. 6 indicates the lines with asymmetrical patterns which Catalán [24] analysed in detail and for which he derived proper $g$ values. Finally, instead of type numbers, the letters $\mathrm{P}-\mathrm{B}$ appear for fifty lines; these are additional lines with Zeeman patterns that 
exhibit partial Paschen-Back effects partially interpreted by Garcia-Riquelme et al. [28]. The Zeeman data were most helpful in this analysis of Mn I; they have confirmed our interpretation almost completely and have permitted the designation of many terms belonging to the doublet system which was the most difficult to establish. The available Zeeman data for $\mathrm{Mn}$ I $[6,21,23,24,27,28,29]$ prove that most of the levels arising from low-energy configurations $\left(d^{5} s^{2}, d^{6} s, d^{5} s p, d^{6} p\right)$ exhibit a remarkably pure LS coupling of electrons, and their $g$-factors are usually identical with the theoretical Landé values, within the error of measurement. Some of the upper levels of both the even and the odd configurations present anomalous $g$-values that may be explained by intermediate coupling or by incorrect grouping of levels in designated terms. Many more Zeeman patterns could be observed if a modern light source that promotes the emission of Mn I radiation were used.

The latest information on spectral terms derived from this analysis of $\mathrm{MnI}$ is presented in table 2 where electron configurations (including series limits), spectral-term designations in standard notation for $L S$ electron coupling, $J$-values, relative numerical values, intervals, and observed $g$-factors are shown in successive columns. The totals are 42 even terms with 125 levels and $60 \mathrm{~g}$ values, and 94 odd terms with 266 levels plus 13 miscellaneous and $164 \mathrm{~g}$ values. In 1952 [21] the totals were 36 even terms with 109 levels and $26 \mathrm{~g}$ values, and 66 odd terms with 214 levels plus 33 miscellaneous and $81 \mathrm{~g}$ values, not counting 12 abandoned levels. It is seen that the present analysis of the Mn I spectrum is a considerable improvement over any preceding, but, if a comparison is made of the presently recognized terms with the table of predicted terms [21, pp. XIV, $\mathrm{XV}]$, any claim that it is satisfactorily completed is unjustified. In order to show the progress that has rewarded this problem during the past 40 years, we briefly summarize in table 3 the number of classified Mn I lines and derived atomic energy levels at different times during this period.

TABLE 3. Energy levels and classified lines of Mn I

\begin{tabular}{|c|c|c|c|}
\hline Year & Reference & $\begin{array}{l}\text { Number } \\
\text { of levels }\end{array}$ & $\begin{array}{l}\text { Number } \\
\text { of lines }\end{array}$ \\
\hline 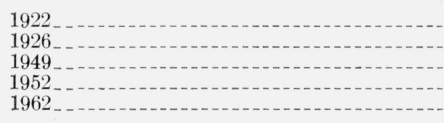 & $\begin{array}{r}4 \\
9 \\
15 \\
21 \\
\text { This work }\end{array}$ & $\begin{array}{r}70 \\
91 \\
211 \\
356 \\
404\end{array}$ & $\begin{array}{r}169 \\
257 \\
711 \\
1500 \\
2030\end{array}$ \\
\hline
\end{tabular}

The present analysis has effectively exhausted all available data on wavelengths, intensitics, and Zeeman patterns of Mn I so that no further progress can be made with that material. But now we know that an arc between manganese electrodes at atmospheric pressure, with which all observations were made, is an inferior light source. Because manganese is a relatively light atom (A-55), and the arc has a high temperature $\left(>5000{ }^{\circ} \mathrm{C}\right)$, it produces broad lines which are always very hazy and unsymmetrical when they involve high energy levels. All complex Mn I terms that converge to $a^{7} \mathrm{~S}_{3}$ or $a^{5} \mathrm{~S}_{2}$ limits in Mn II have very small intervals and are mostly unresolved because of the excessive line widths. Furthermore, the traditional are in air always produces a strong background of molecular spectra that often masks atomic lines. All of these spectroscopic objections to an are in air can now be avoided by using highly evacuated electrodeless (quartz tube) lamps containing a trace of metal or halide compound excited with ultrahigh frequency as demonstrated by Meggers and Westfall [33] for mercury and by Corliss and Meggers [34] for hafnium. At elevated temperatures (ca. $800{ }^{\circ} \mathrm{C}$ ) such lamps favor spectra of neutral atoms, especially important for faint lines and for observing first spectra in strong magnetic fields; we mention this to aid anyone who becomes ambitious to make further progress in the description and quantum interpretation of the Mn I spectrum. That such progress is desirable becomes obvious when the now known spectral terms (table 2) are compared with the predicted ones [21]. Although many electron configurations and spectral terms have been identified, rarely is a configuration represented by all its terms and in some cases the terms themselves are fragmentary or even uncertain.

In 1922, Catalán derived, from spectral series of running $s$ electrons, the absolute energy of the ground state of Mn I, and computed the first ionization potential to be 7.41 electron volts. Thirty years later Catalán and Velasco [35] systematically studied the first three ionization potentials of elements in the iron group; for Mn I they obtained an absolute value of $59960 \mathrm{~K}$ for the ground state, the equivalent $7.432 \mathrm{eV}$. These values were quoted in the second volume of Atomic Energy Levels [21]. A decade later, Garcia-Riquelme [36] applied a Ritz formula to a series of ${ }^{6} \mathrm{~F}$ terms with four members (4f, $5 \mathrm{f}, 6 \mathrm{f}$, 7f) and found the limit near $59979 \mathrm{~K}$ or $7.434 \mathrm{eV}$. We decided to adopt the average of the last two determinations resulting in a limit of $59970 \mathrm{~K}$ or ionization potential of $7.433 \mathrm{eV}$ for normal manganese.

The surviving authors wish to thank R. Velasco for valuable assistance in the term analysis of the Mn I spectrum, especially with the measurement and interpretation of Zeeman patterns published elsewhere. We are also very grateful to Mrs. Charlotte Moore-Sitterly whose experience and judgment in spectral-term analyses were frequently consulted. We are deeply indebted to Mrs. Isabel Murray for making a square array of all possible combinations of Mn I energy levels, and for preparing the final tables; her work is unsurpassed in accuracy and neatness. Without the aids above, the publication of this paper as a token of our affection and esteem for the late Professor Catalán might have been indefinitely delayed. 


\section{References}

[1] H. Kayser, Handbuch der Spectroscopie, 5, 726-771; 1910.

[2] H. Kayser and H. Konen, Handbuch der Spectroscopie \%, 1052-1108; 1934 .

[3] H. Kayser and C. Runge, Über die Spectra von Zinn, Blei, Arsen, Antimon und Wismuth, Wiedem. Ann., 5\%, 93-113; 1894.

[4] M. A. Catalán, Series and Other Regularities in the Spectrum of Manganese, Phil. Trans. Roy. Soc. London, 223, A, 127-173; 1922 .

[5] A. Sommerfeld, Über die Deutung verwickelter Spektren (Mangan, Chrom, usw.) nach der Methode der inneren Quantenzahlen. Ann. d. Physik, 70, 32-62; 1923.

[6] E. Back, Der Zeeman effekt des Bogen-und Funkenspektrums von Mangan. Z. Physik, 15, 206-243; 1923.

[7] A. Landé. Termstruktur und Zeeman effekt der Multiplets. Z. Physik, 15, 189-205; 1923.

[8] M. A. Catalán. Los Espectros y la Clasificacion Periodica. Anales Soc. Españ. Fis. y Quim. 21, 321-329; 1923.

[9] J. C. MeLennan and A. B. MeLay. On the Structure of the Arc Spectrum of Manganese. Trans. Roy. Soc. Can. 20, 89-120, 1926.

[10] H. N. Russell. Series and Ionization Potentials of the Elements of the Iron Group. Astrophys. J., 66, 233$255 ; 1927$.

[11] M. A. Catalán. Notas sobre la estructura del espectro del Manganeso. Anales Soc. Españ. Fis. y Quim., 26, 67-74; 1928.

[12] R. F. Bacher and S. Goudsmit. Atomic Energy States as derived from Analyses of Optical Spectra. pp. 278281, McGraw-Hill Book Co., New York, 1932.

[13] T. Dunham, Jr., Unpublished multiplets of Mn I.

[14] W. F. Meggers. The infrared Arc Spectra of Manganese and Rhenium. J. Res. Nat. Bur. Std. 10, 757-769; 1933.

[15] O. Garcia-Riquelme. Estructura y nuevos niveles energeticos del espectro arco del manganeso. Anales Soc. Españ. Fis. y Quim., 45A, 435-448, 547-576; 1949.

[16] H. Slevogt. U̇ber die Bogenspektren von Chrom, Mangan, Kobalt und Nickel im Rot und nahen Ultrarot. Z. Physik, 82, 92-118; 1933.

[17] F. W. Paul. Absorption Spectra of Manganese and Silver in the Schumann Region. Phys. Rev., 52, 923-929; 1937.

[18] A. G. Shenstone. The First Spark Spectrum of Platinum. Phil. Trans. Roy. Soc. London, 237, 453-470; 1938.

[19] R. W. Wood. Recent Improvements in Diffraction Gratings and Replicas. Nature 140, 723-724; 1937.
[20] H. M. Randall and E. F. Barker. The Infrared Arc Spectra of Cobalt, Nickel, Manganese and Chromium Astrophys. J., 49, 59-63; 1919.

[21] C. E. Moore. Atomic Energy Levels as Derived from the Analyses of Optical Spectra. N.B.S. Cir. 467, vol. 2, 27-31; 1952 .

[22] G. R. Harrison and F. Bitter. Zeeman Effects in Complex Spectra at Fields up to 100,000 Gauss. Phys. Rev., 5\%, 15-20; 1940.

[23] M. A. Catalán and R. Velasco. Changes in $g$-values in a Spectrum, for different Magnetic Fields. J. Opt. Soc. Am., 40, 653-655; 1950.

[24] M. A. Catalán. Asymmetries of Zeeman Patterns and $g$-values for Neutral Manganese. J. Res. Nat. Bur. Std., 47, 502-524; 1951.

[25] M. L. Espinosa. Estudio de Patrones de fuerte interaccion Paschen-Back. Anales Soc. Españ. Fis. y Quim., 48A, 211-218; 1952.

[26] M. L. Espinosa. Interpretacion del Multiplete $a^{6} \mathrm{~S}-$ $z^{6} \mathrm{P}^{0}$ del Mn I en campo magnetico del orden de los 85,000 oersted. Anales Soc. Españ. Fis. y Quim. 48A, $267-270 ; 1952$.

[27] M. A. Catalán and O. Garcia-Riquelme. Medidas de efecto Zeeman en el espectro de Mn I. Anales Soc. Españ. Fis. y Quim. 47A, 173-180; 1951.

[28] O. Garcia-Riquelme, R. Velasco, R. Oyarzun, and F. Veas. Nuevas interpretaciones en el efecto Zeeman de Mn r. Anales Soc. Españ. Fis. Quim. 56A, 137$150 ; 1960$.

[29] O. Garcia-Riquelme. Unpublished Zeeman data for Mn I. June 1962.

[30] H. Kayser. Tabelle der Schwingungszahlen. S. Hirzel, Leipzig, 1925.

[31] C. D. Coleman, W. R. Bozman, and W. F. Meggers. Table of Wave numbers. N.B.S. Monograph 3. Government Printing Office, Washington, 1960.

[32] E. Back and A. Landé. Zeemaneffekt und Multiplett struktur der Spektrallinien. pp. 168-169. Julius Springer, Berlin, 1925.

[33] W. F. Meggers and F. O. Westfall. Lamps and wavelengths of Mercury 198. J. Res. Nat. Bur. Std. 44, $447-455 ; 1950$.

[34] C. H. Corliss and W. F. Meggers. Improved Description of Hafnium Spectra. J. Res. Nat. Bur. Std. 61, 269$324 ; 1958$.

[35] M. A. Catalán and R. Velasco. Series y potenciales de, ionizacion en los espectros I, II, y III de los elementos del grupo del hierro. Anales Real Soc. Espan. Fis. Quim. 68A, 247-266; 1952 .

[36] O. Garcia-Riquelme, Unpublished material, 1962.

(Paper 68A1-252) 